\title{
Active Emulsions
}

Physicochemical Hydrodynamics and Collective Behavior

\author{
Dissertation \\ For THE AWARD OF THE DEGREE \\ "Doctor of Philosophy" \\ of the University of Göttingen \\ within the doctoral program \\ in physics \\ of the Georg-August University School of Science (GAUSS) \\ by

\section{Babak Vajdi Hokmabad} \\ from \\ Tabriz, Iran
}

Göttingen, 2020 


\section{Thesis Committee}

Dr. Corinna MaAss,

Department of Complex Fluids,

Max Planck Institute for Dynamics and Self-Organization

Dr. Christian BAhr,

Department of Complex Fluids,

Max Planck Institute for Dynamics and Self-Organization

Prof. Dr. Annette Zippelius,

Institute for Theoretical Physics,

Georg-August-Universität Göttingen

\section{Members of the Examination Board}

Referee: Dr. Corinna MaAss,

Department of Complex Fluids,

Max Planck Institute for Dynamics and Self-Organization

$2^{\text {nd }}$ Referee: Prof. Dr. Stefan Klumpe,

Institute for the Dynamics of Complex Systems,

Georg-August-Universität Göttingen

\section{Further members of the Examination Board:}

Prof. Dr. Jörg ENDERLEIN,

III. Physical Institute,

Georg-August-Universität Göttingen

Prof. Dr. Annette Zippelius,

Institute for Theoretical Physics,

Georg-August-Universität Göttingen

Dr. Christian Bahr,

Department of Complex Fluids,

Max Planck Institute for Dynamics and Self-Organization

Dr. Stefan KarpitschKa,

Department of Complex Fluids,

Max Planck Institute for Dynamics and Self-Organization

Date of oral examination: 2021-01-19 
This thesis is dedicated to my family Afsaneh, Aliasghar and Siamak. 



\begin{abstract}
Active matter is a collection of constituent elements that constantly consume energy, convert it to mechanical work, and interact with their counterparts. These materials operate out of equilibrium and exhibit fascinating collective dynamics such as spontaneous pattern formation. Self-organization of bio-polymers within a cell, collective migration of bacteria in search of nutrition, and the bird flocks are paragons of active living matter and the primary source of our knowledge on it. To understand the overarching physical principles of active matter, it is desirable to build artificial systems that are capable of imitating living active matter while ruling out the biological complexities.

The goal of this thesis is to study active micro-droplets as a paradigm for biomimetic artificial active particles, using fundamental principles of fluid dynamics and statistical physics. The Marangoni-driven motility in these droplets is reminiscent of the locomotion of some protozoal organisms, known as squirmers. The main scientific objectives of this research are to (i) investigate the potential biomimetic features of active droplets including compartmentalization, adaptability (e.g. multi-gait motility), and information processing (signaling and sensing) and (ii) study the implications of those features in the collective dynamics of active emulsions governed by hydrodynamic and autochemotactic interactions. These objectives are addressed experimentally using microfluidics and microscopy, integrated with quantitative image analysis. The quantitative experimental results are then compared with the predictions from theory or simulations. The findings of this thesis are presented in five chapters.

First, we address the challenge of compartmentalizing active droplets. We use microfluidics to generate liquid shells (double emulsions). We propose and successfully prove the use of a nematic liquid crystal oil to stabilize the liquid shells, which are otherwise susceptible to break up during motility. We investigate the propulsion dynamics and use that insight to put forward routes to control shell motion via topology, chemical signaling, and topography.

In the second results chapter, we establish the bimodal dynamics of chaotic motility in active droplets; a regime that emerges as a response to the increase of viscosity
\end{abstract}


in the swimming medium. To establish the physical mechanism of this dynamical transition, we developed a novel technique to simultaneously visualize the hydrodynamic and chemical fields around the droplet. The results are rationalized by quantitative comparison to established advection-diffusion models. We further observe that the droplets undergo self-avoiding random walks as a result of interaction with the selfgenerated products of their activity, secreted in the environment.

The third results chapter presents a review of the dynamics of chemotactic droplets in complex environments, highlighting the effects of self-generated chemical interactions on the droplet dynamics.

In the fourth results chapter, we investigate how active droplets sense and react to the chemical gradients generated by their counterparts - a behavior known as autochemotaxis. Then, we study the collective dynamics governed by these autochemotactic interactions, in two and three dimensions. For the first time, we report the observation of 'history caging', where swimmers are temporarily trapped in an evolving network of repulsive chemical trails. The caging results in a plateau in the mean squared displacement profiles as observed for dense colloidal systems near the glass transition.

In the last results chapter, we investigate the collective dynamics in active emulsions, governed by hydrodynamic interactions. We report the emergence of spontaneously rotating clusters. We show that the rotational dynamics originates from a novel symmetry breaking mechanism for single isotropic droplets. By extending our understanding to the collective scale, we show how the stability and dynamics of the clusters can be controlled by droplet activity and cluster size.

The experimental advancements and the findings presented in this thesis lay the groundwork for future investigations of emergent dynamics in active emulsions as a model system for active matter. In the outlook section, we present some of the new questions that have developed in the course of this research work and discuss a perspective on the future directions of the research on active droplets. 


\section{Table of contents}

1 Introduction $\quad 1$

1.1 Hallmarks of living matter . . . . . . . . . . . . . . . . . 3

1.2 Swimming at low Reynolds number . . . . . . . . . . . . . 5 5

1.3 Motile biomimetic microswimmers . . . . . . . . . . . . . . 8

1.3.1 Autophoretic active particles . . . . . . . . . . . . . 10

1.3.2 Mechanisms for symmetry breaking . . . . . . . . . . . . . 13

1.3.2.1 Intrinsic symmetry breaking . . . . . . . . . . . . . 13

1.3.2.2 Spontaneous symmetry breaking . . . . . . . . . 14

1.3.3 Active droplets . . . . . . . . . . . . . . . 15

1.4 Collective dynamics in active matter . . . . . . . . . . . . 20

1.4.1 Collective dynamics governed by short-range interactions . . . . 21

1.4.2 Collective dynamics governed by long-range interactions . . . . 21

1.4.2.1 Chemical interactions . . . . . . . . . . . . 22

1.4.2.2 Hydrodynamic interactions . . . . . . . . . . . 23

1.5 Scope of this Thesis . . . . . . . . . . . . . . . . . . 23

1.6 Structure of the thesis . . . . . . . . . . . . . . . . . 25

2 Topological stabilization and dynamics of self-propelling nematic $\begin{array}{ll}\text { shells } & 29\end{array}$

2.1 Introduction . . . . . . . . . . . . . . . . . . . 30

2.2 Results and discussion . . . . . . . . . . . . . . . . . . . 30

2.3 Conclusion . . . . . . . . . . . . . . . . . . 36

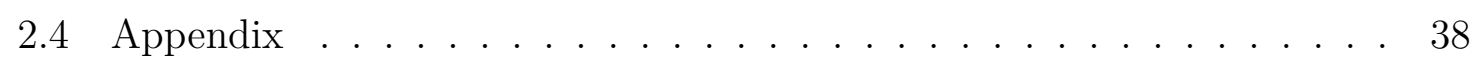

2.4 .1 Double emulsion fabrication . . . . . . . . . . . . 38

2.4 .2 Observation and analysis . . . . . . . . . . . . . . . 39

2.4.3 Numerical simulation . . . . . . . . . . . . . . . . . . . . 41

3 Emergence of bimodal motility in active droplets 45 
3.1 Introduction . . . . . . . . . . . . . . . . 46

3.2 Droplets propelled by micellar solubilisation . . . . . . . . . . . . . 47

3.3 Simultaneous visualisation of chemical and hydrodynamic fields . . . . 49

3.4 Destabilised motion with increasing Péclet number . . . . . . . . . . . 49

3.5 Signatures of unsteady dynamics in the time evolution of chemical and hydrodynamic fields . . . . . . . . . . . . . . . . 52

3.6 Dependence of hydrodynamic modes on the Péclet number . . . . . . . 54

3.7 Interactions with self-generated chemical gradients cause speed bursts . 56

3.8 Consequences for spatial exploration . . . . . . . . . . . . 58

3.9 Appendix . . . . . . . . . . . . . . . . . . . . 60

4 Chemotactic droplet swimmers in complex geometries $\quad 75$

4.1 Introduction . . . . . . . . . . . . . . . . . . . . 76

4.2 Interfacial interactions and chemotaxis in microswimmers . . . . . . . . 77

4.3 Active liquid crystal emulsions . . . . . . . . . . . . . . . . . . . . . . 79

4.4 Methods . . . . . . . . . . . . . . . . . . . . 81

4.5 Chemotaxis and autochemotaxis . . . . . . . . . . . . . . 82

4.5 .1 Maze solving . . . . . . . . . . . . . . . . . 84

4.5.2 Diffusion controlled autochemotaxis . . . . . . . . . . . 85

4.6 Chemotactic effects at pillars . . . . . . . . . . . . . 87

4.7 Conclusion . . . . . . . . . . . . . . . . . . . . . . 93

5 Chemorepulsive alignment-induced interactions in active emulsions 95

5.1 Introduction . . . . . . . . . . . . . . . . . . . . . . 96

5.2 Methods and general swimming behaviour . . . . . . . . . . . . . . . 97

5.3 Chemically Active Polar Particle model . . . . . . . . . . . . . . . . . . 99

5.4 Experimental quantitative analysis of droplet-trail interactions in 2D $\quad 100$

5.5 Fits of model and experiment droplet-trail interactions . . . . . . . . . 102

5.6 Phase diagram using results of the fit . . . . . . . . . . . . . . . . . . 104

5.7 Collective dynamics governed by autochemotactic interactions: history caging . . . . . . . . . . . . . . . . . . 105

5.8 Extending the system to 3D . . . . . . . . . . . . . . . . 107

5.9 Conclusion and outlook . . . . . . . . . . . . . . . 108

5.10 Materials and Methods . . . . . . . . . . . . . . . . . . 110

5.10 .1 Materials and characterisation . . . . . . . . . . . . 110

5.10 .2 Micellar diffusion quantification by fluorescence . . . . . . . . . 111 
5.10.3 Binary collisions: Image processing and data analysis . . . . . . 111

5.10 .4 Laser sheet fluorescent microscopy . . . . . . . . . . . . . . . . . 112

6 Spontaneously rotating clusters of active droplets 117

6.1 Introduction . . . . . . . . . . . . . . . . . . . . 118

6.2 Attractive hydrodynamic interaction between droplets . . . . . . . . . . 118

6.3 Formation of spontaneously-rotating clusters . . . . . . . . . . . . . . . 119

6.4 Phase diagram for the cluster dynamics . . . . . . . . . . . . . . . 121

6.5 Rotational broken symmetries in individual droplets . . . . . . . . . . 123

6.6 Discussion and Conclusion . . . . . . . . . . . . . . . . . 125

6.7 Appendix ........................ 126

$\begin{array}{lll}7 & \text { Discussion and Outlook } & 127\end{array}$

References 



\section{Chapter 1}

\section{Introduction}

We have all been fascinated by the mesmerizing scene of bird flocks flying around in harmony. As explored more in nature we were able to find more examples of such large-scale pattern formation in fish schools [1], swarms of midges [2, 3], bacterial communities [4], and even human crowds [5]. While the curiosity to understand these patterns is as old as human history, we have only recently been able to develop tools and techniques that can help us systematically observe them and quantify their dynamics.

Discovering the striking similarities in the phenomenology and even mechanisms of various types of pattern formation have led to the emergence of a new research field known as active matter. Active matter is a collection of active "agents" that consume energy, convert it to mechanical work (e.g. movement), and interact with their counterparts. Such a definition will encompass a broad spectrum from actin filaments [6] and microtubules in motility assays [7] to bacteria [4] and all the way to the bird flocks $[8,9]$.

Due to continuous injection of energy, active matter systems operate out of thermodynamic equilibrium which means the thermodynamic properties of the system vary temporally and spatially. The spatial gradients in those properties result in the transport of mass, momentum, and energy [9, 10]. A thermodynamic equilibrium will not be reached unless access to the source of energy is lost. What distinguishes active matter from other non-equilibrium systems is the local (microscopic) dissipation of energy. The energy input for the system is done through individual constituent elements, hence, each element has irreversible dynamics [9, 10].

While many studies in the field are still curiosity-driven, we have come to realize the significance of understanding active matter physics by putting it into bigger contexts. In particular, the micro-scale active matter is proven to be of great importance. For 
instance, biofilm formation in bacterial communities leads to contamination in coronary implants and water transport systems $[11,12]$ and collective migration of pelagic phytoplankton plays a role in the energy transfer into the biosphere [13]. It has also shown great promise in creating novel approaches to extract work in micro-scale systems [14] or developing new functional bio-materials with reduced viscosity $[15,16]$.

Emergent complexity in active matter dynamics is intimately related to the mutual interactions between the constituents $[9,10]$. These interactions can be of a purely physical origin, such as steric or hydrodynamic interactions, or of biological nature, like visual signals or quorum sensing [17].

Aiming for universality, most of the theoretical attempts are on generic and simplified models- in terms of shape and interaction rules- of the active constituents. On the other hand, the majority of the experimental work is focused on pattern formation in micro-scale and on micro-organisms. The reason, beyond their particular biophysical importance per se, is that they are the simplest form of living systems that are also available in large numbers and detectable via our present technological capabilities. However, even for the simplest forms of micro-organisms, it is a great challenge to distinguish and separate the purely physical interaction from the more biologicallyrelated ones and no theoretical model can include all these nuances that exist within a multitude of length and time scales. Hence, we need to develop an experimental model system that is controllable, adaptive and works on the basis of simple principles of physical chemistry. Such a system can be used as a benchmark for direct comparison with computer simulations and theoretical predictions. In the absence of biological complexity, we can rule out different possible inter-particle interactions and concentrate on pattern formation based on a single type of interaction and step-by-step add on different scenarios that eventually lead to emergent complexity.

Once we have gained the knowledge to describe the dynamics of the model system, we can benefit from it in two ways. First, development of non-equilibrium micromachines with embedded functionalities e.g. cargo transport or sensing. Second, we can study fundamentals of active matter with protocells with designs that are gradually evolving to incorporate more biomimetic features.

In the next section, we will present some of the fundamental concepts in biomimetic active matter. 


\subsection{Hallmarks of living matter}

Living systems, as we know them, are results of billions of years of evolution and we are at the beginning of a long way to understand their complex nature. Despite the immense emergent diversity and complexity in living systems, there are characteristics that are common between all forms of life [18]. Advancements in cell biology have enabled us to explore and understand the simplest forms of life. This knowledge of how the simplest living systems work can inspire us to design and engineer biomimetic artificial systems from the bottom up. In the following, we will discuss hallmarks of simple living systems: Compartmentalisation, Growth and division, Information processing, Energy transduction, and Adaptability, as summarized in Fig. 1.1.

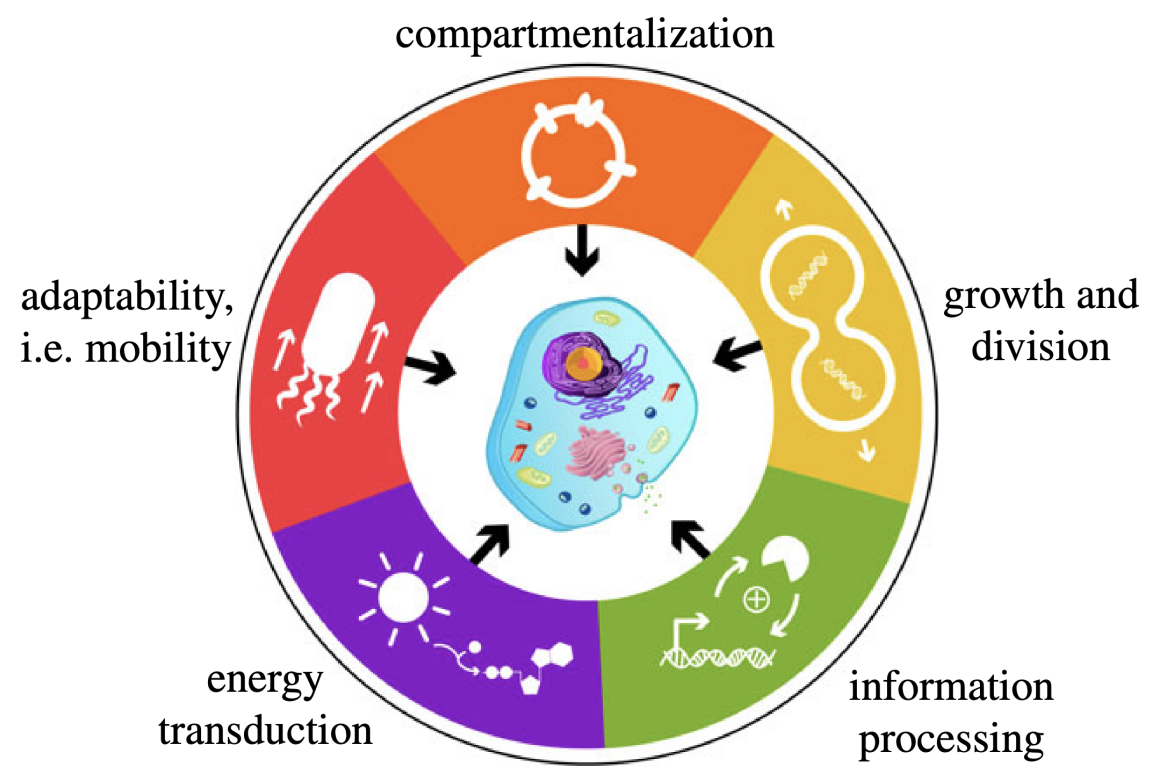

Figure 1.1 - The hallmarks of living systems. Taken from [18] with permissions.

Compartmentalization is critical to separate the components of the living system from the environment. It spatially concentrates reactants and chemical networks and confines the genetic material of the cell. In cells, this semi-permeable membrane is responsible for compartmentalisation. It regulates the communication of the cell with the environment enabling the cell to create spatiotemporal chemical gradients that are critical for functioning out of equilibrium. 
Growth and division: The generation of new cells is done through growth and subsequent self-replication. To this end, the compartments are supposed to regulate the spatiotemporal dynamics of growth, deformation, and eventually division. Living cells undergo this process through complex dynamics in their cytoskeleton [19].

Information processing: Living systems are able to sense, process, and respond to information (signals). The source of the signals can be internal or external. At the intracellular level, this function is primarily associated with processing the genetic information from carrier molecules e.g. DNA and RNA. The cell also responds to extracellular information including environmental cues as well as intercellular communication. Through their compartment interface (i.e. the membrane), cells sense these cues from their surroundings, communicate with other cells, and adapt their behavior accordingly.

Energy transduction: As systems that are operating out of equilibrium, cells need to harness energy from external sources to be able to run the biochemical reactions necessary for their activity i.e. metabolism. Cells receive the energy from their environment and convert it into energy currencies, such as ATP molecules, which then spread in the cell body powering the metabolic chemical reactions.

Adaptability: The adaptability of a living system to variations in the environment is critical for its survival and, in turn, its evolution [20]. An important and ubiquitous example of adaptability is motility in living systems. It is motivated by their need to move towards energy sources, or interact with interfaces, e.g. in biofilm formation. In the cells' case, they have evolved to process environmental cues, identify energy sources, and respond to them by directing/biasing their motion accordingly. Examples are directing movement towards sources of light (phototaxis) [21] or chemical substances, "food", (chemotaxis) [22], following topology (topotaxis) [23] or magnetic fields (magnetotaxis) [24], and active reorientation with respect to gravity or external flows (gravitaxis [25] and rheotaxis [26, 27], respectively).

To perform self-propulsion, an entity has to convert its consumed energy into kinetic energy (in the form of a movement of the entire or a part of the body). Since cells consume energy and convert it to mechanical work at an individual level, one can study them as active matter when they form a community $[9,28,29]$. 
While far from developing an artificial system capable of mimicking all hallmarks of life, science has made great progress in building systems that emulate one or combine a few characteristic features of life. Bottom-up approaches, successful in developing such systems, have employed the principles of physical chemistry within a new context: developing adaptable self-sustaining systems that function out of equilibrium. Building minimal model systems that show life-like behavior will inform us about the underlying physicochemical rules that drive actual biological systems and step-by-step, direct us towards more complicated designs for biomimetic active matter. This thesis aims to develop an artificial model system for motile active particles and investigate its biomimetic features. The first challenge for a motile particle is to overcome the fluidic constraints. Thus, in the next section, we discuss the physics of swimming at very small small scales.

\subsection{Swimming at low Reynolds number}

To describe the flow and obtain the force distribution on an object moving inside a fluid, one needs to solve the Navier-Stokes equation. The solution for the velocity field $\mathbf{u}(\mathbf{r}, t)$ and pressure field $p(\mathbf{r}, t)$ for an incompressible Newtonian fluid flow satisfies the

$$
\rho\left(\frac{\partial}{\partial t}+\mathbf{u} . \nabla\right) \mathbf{u}=-\nabla p+\eta \nabla^{2} \mathbf{u},
$$

and the continuity equation

$$
\nabla \cdot \mathbf{u}=0
$$

where $\rho$ and $\eta$ are density and viscosity of the fluid, respectively. Navier-Stokes and continuity equations mathematically express the conservation of momentum and mass. Identifying the boundary conditions and making appropriate assumptions for them are critical to acquiring an accurate solution to the problem. Once $\mathbf{u}$ and $p$ are known, one can find the stress tensor, and subsequently, through integration over the surface of the object, the hydrodynamic force and torque can be obtained.

The dimensionless quantity that characterizes the flow regime described and solved by the Navier-Stokes equation is the Reynolds number. For a steady flow with velocity $U$ and characteristic length scale $L$, the Reynolds number is defined as the ratio of the inertial term, $\sim \rho \mathbf{u} \cdot \nabla \mathbf{u}$, to the viscous term, $\sim \eta \nabla^{2} \mathbf{u}$, in the Navier-Stokes equation [30]. Hence, 


$$
R e=\frac{\rho U L}{\eta} .
$$

There are two other very useful interpretations of the Reynolds number. The first one is based on the ratio of the relevant time scales. Re can be defined as the ratio of the advective time scale for transport of a velocity perturbation, $t_{\mathrm{adv}} \sim L / U$, to the time scale for this perturbation to diffuse away, $t_{\text {diff }} \sim \rho L^{2} / \eta$. Thus, $R e=t_{\text {diff }} / t_{\text {adv }}$ will give the same definition as above. The second interpretation arises from the ratio of forces acting on the body of an object. The viscous and inertial forces acting on a bluff body have the form of $f_{\text {viscous }} \sim \eta U L$ and $f_{\text {inertial }} \sim \rho U^{2} L^{2}$, respectively. The third definition of $R e=f_{\text {inertial }} / f_{\text {viscous }}$ again leads to the same simple form shown above.

$R e$ can characterize a broad spectrum of flow regimes i.e. if they are laminar or turbulent. The critical values, can be used universally to predict the dynamical transition in the flow behavior. For example, for the flow generated by human breaststroke, which is turbulent, $R e=\mathcal{O}\left(10^{4}\right)$. In the world of micro-swimmers, such as the bacterium $E$. coli, the swimming ( $U \approx 10 \mu m$ and $L \approx 1-10 \mu m$ ) has a Reynolds number of $R e=\mathcal{O}\left(10^{-5}-10^{-4}\right)$. At such low $R e$, the inertial effects are negligible and the viscous damping is dominant. Thus, it is possible to study them at the limit of $R e=0$. This assumption reduces the Navier-Stokes equation to the Stokes equation:

$$
-\nabla p+\eta \nabla^{2} \mathbf{u}=0, \quad \nabla \cdot \mathbf{u}=0
$$

The fact that these equations are time-invariant and linear has significant implications in the locomotion of micro-swimmers. For example, if a swimming E. coli suddenly stopped its activity, it would only coast a distance in the order of $0.1 \AA$ and then stop [31, 32]. Another well-known example is known as Purcell's scallop theorem [31]. A scallop moves by opening its shell slowly and closing it very quickly so that a jet of fluid is pushed out. Purcell argued that this mechanism has to be operated in an inertial flow regime. Otherwise, since at the low Re regime the flow is time-reversible, the reciprocal movement of the shell cannot lead to a net propulsion. This underscores the importance of non-reciprocal movements to break the symmetry and thereby achieve a directional self-propulsion.

Since the inertia-based swimming mechanisms used by larger animals are not applicable at low $R e$ regime, micrometer-sized biological swimmers have evolved to develop propulsion mechanisms that break the symmetry via non-reciprocal movements. The motility of many micro-swimmers depends on one or more appendages that are 
connected to molecular motors that perform the mechanical work. A few examples are presented in Fig. 1.2. Sperm is a mono-flagellated example that swims via whip-like movements of a flexible filament at its rear side. E. coli is a well-studied organism with multiple flagella that are connected to a rotary motor, embedded in the cell body. Once the motors rotate, the left-handed helical flagella bundle and generate thrust which pushes the body forward. Chlamydomonas reinhardtii, a green alga which can be found in wet soil, is a biflagellated organism. The flagella, located at the front of the cell, perform a non-reciprocal breaststroke, and pull the body forward. A rather big organism called Paramecium uses a spectacular method to generate thrust. Its body is covered with flexible cilia that are considerably shorter compared to body size. These cilia synchronously beat, resulting in a net drag force that propels the body.

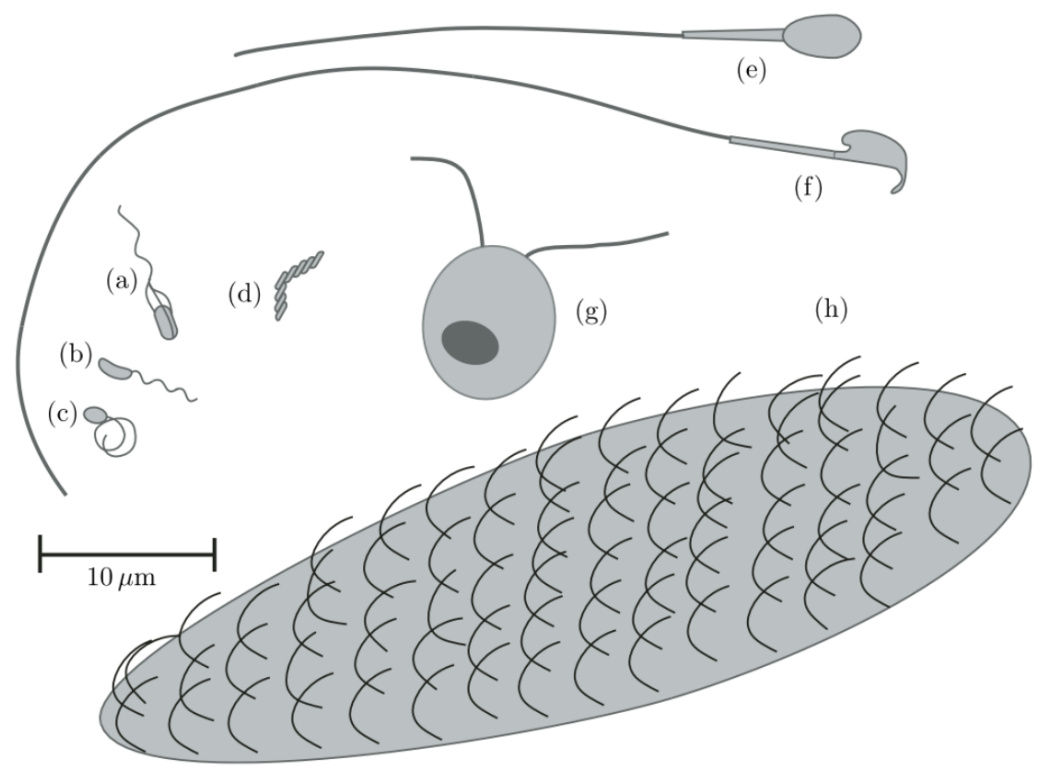

Figure 1.2 - Sketches of biological micro-swimmers, to scale. (a) E. coli. (b) C. crescentus. (c) R. sphaeroides, with flagellar filament in the coiled state. (d) Spiroplasma, with a single kink separating regions of right-handed and left-handed coiling. (e) Human spermatozoon. (f) Mouse spermatozoon. (g) Chlamydomonas. (h) A smallish Paramecium. from [30] with permissions.

A well-established approach to categorize the biological micro-swimmers is based on the type of far-field flow that the organism generates. The squirmer is a model for spherical microswimmers that propel by a purely tangential velocity on their surface. The velocity at a point $\mathbf{r}_{\mathrm{s}}$ on the surface of a squirmer of radius $a$ is given by [33]

$$
\mathbf{v}_{\mathrm{s}}\left(\mathbf{r}_{\mathrm{s}}, \hat{\mathbf{e}}\right)=\sum_{n=1}^{2} \frac{2}{n(n+1)} B_{n}\left(\frac{\hat{\mathbf{e}} \cdot \mathbf{r}_{\mathrm{s}}}{a} \frac{\mathbf{r}_{\mathrm{s}}}{a}-\hat{\mathbf{e}}\right) P_{n}^{\prime}\left(\hat{\mathbf{e}} \cdot \mathbf{r}_{\mathrm{s}} / a\right)
$$


where $B_{n}$ are constants, $P_{n}^{\prime}(x)$ is the derivative of the $n$th Legendre polynomial, $\hat{\mathbf{e}}$ is a unit direction vector. Usually, a good approximation of the resulting flow field, in the far-field, is already provided by the first two modes of the polynomial expansion. Then, Eqn. (1.5) gives the surface velocity as $u_{\theta}=B_{1} \sin \theta+0.5 B_{2} \sin 2 \theta$ where $\theta$ is the polar angle. The relative strengths of $B_{1}$ and $B_{2}$ known as the squirmer parameter $\beta \equiv B_{2} / B_{1}$ determines the characteristics of the surface velocity. For $\beta \neq 0$, two distinct types of far-field flow are generated by squirmers: pusher type and puller type.

\section{Pusher micro-swimmers}

When $\beta<0$ the generated flow has a pusher form. $E$ coli is an example organism that generates a pusher type flow field. When it is moving forward, the flagella bundle at its rear side rotate to push the body forward, generating a flow field visualized by Drescher et al. [34] (Fig. 1.3 a). It is called a pusher since the distinguishing feature is that the fluid is pushed away from the swimmer at its front and rear. Consequently, the fluid is pulled towards the body from the sides of the swimmer.

\section{Puller micro-swimmers}

When $\beta>0$ the generated flow has a puller form. Chlamydomonas reinhardtii is an example organism that generates a puller type flow field. The body is pulled by the two flagella located at its front. The flagella beat in a breaststroke-like pattern, resulting in the thrust that propels the organism forward. The generated hydrodynamic flow field is shown in Fig. $1.3 \mathrm{~b}$ ). It is called a puller due to the fact that the fluid is pulled towards the swimmer at its front and rear. Thus, the outward flow streams occur at the sides of the swimmer.

\subsection{Motile biomimetic microswimmers}

Artificial micro-swimmers have been designed and manufactured to reproduce certain features of motile microorganisms. They can be building blocks of synthetic active matter; a system consisting of these artificial constituents that can emulate collective dynamics, observed in nature, in a controlled setting, and even be programmed to produce new dynamics. The swimmers can also be used as micro-machines engineered for transport or sensing applications. They can be categorized based on the type of energy input and the strategy for self-propulsion. 

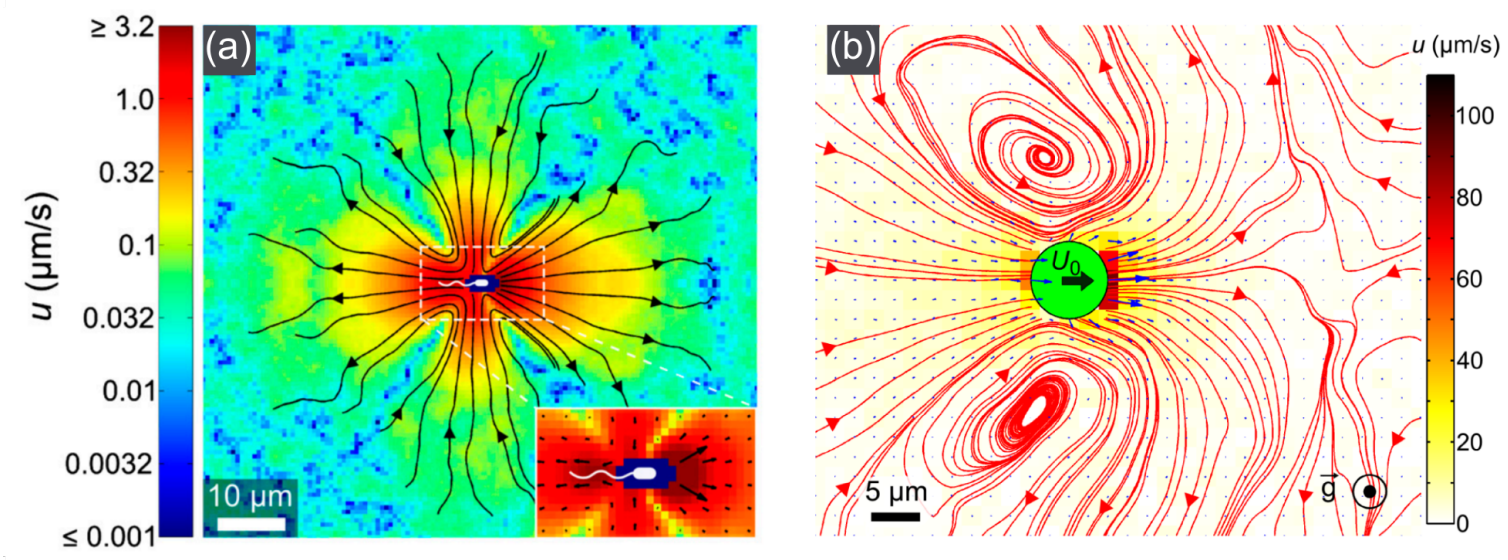

Figure 1.3 - Mean flow field generated by a single freely swimming (a) E. coli as a pusher swimmer (from [34] with permissions), and (b) C. reinhardtii as a puller swimmer (from [35] with permissions).

\section{Energy input: internal vs. external}

Particles can be driven by external fields such as magnetic, electric, and acoustic [36]. The advantage of this approach is the ability to control the motion speed and direction via modulating the strength and direction of the external field. For example, a ferromagnetic colloid in an external alternating magnetic field shows a rolling motion which is driven directly by the force induced by the rotating field. [37, 38].

In contrast, there are active particles that are driven out of equilibrium through local conversion of energy (e.g., catalytic processes). The best known method to induce propulsive force onto colloid is phoretic transport. By creating a self-made gradient in chemical, electrostatic, or thermal field around the particle an autophoretic motion takes place. It will be discussed in detail in Section 1.3.1. For example, Janus micro-swimmers use an asymmetric chemical reaction catalyzed on their own surface to achieve self-propulsion [39-41].

\section{Micro-swimmers with or without moving parts}

Artificial microswimmers that are driven mechanically use moving parts that mimic biological counterparts in performing non-reciprocal movements. The first example of such systems was the linear chains of DNA-linked magnetic colloids that were attached to a red blood cell [42]. The flexible synthetic flagellum was actuated by an external oscillating magnetic field. The manufacturing of such designs is challenging and they are prone to fatigue failure. 
On the other hand, most of the synthetic micro-swimmers are able to self-propel without any moving parts. For the case of self-driven swimmers, the above-mentioned autophoresis method is broadly used.

\subsubsection{Autophoretic active particles}

In classical fluid mechanics, the boundary conditions at the interface are imposed by the continuity of velocity and stress. However, it has been shown that an interface has an infinitesimal but finite thickness and dynamical transport processes can take place in there, resulting in discontinuities in velocity and stress at the interface [43]. These interfacial forces can actually be exploited to induce colloidal transport.

Consider a small spherical droplet inside a second fluid with a gradient in the concentration of a chemical species (e.g. a molecular solute). Assume the solute is insoluble in the droplet and it can change the interfacial tension $\gamma$ at the droplet interface and both liquids have the viscosity of $\eta$. If the droplet undergoes a drift with a velocity $U$, the solute gradient on the droplet interface generates a front-aft interfacial tension gradient that propels the droplet forward. By balancing viscous dissipation and the work by surface tension gradients we can obtain $U \propto \Delta \gamma / \eta$. $\Delta \gamma$ is the first order approximation for front-aft interfacial tension gradient. Assuming a viscosity close to water's $\eta \sim 10^{-3}$ Pa.s, a very small interfacial tension gradient of $\sim 10^{-9} \mathrm{~N} / \mathrm{m}^{2}$ is enough to move the droplet with a speed of $1 \mu \mathrm{m} / \mathrm{s}$. Experiments have reported considerably larger magnitudes for velocity $\mathcal{O}(10-100 \mu \mathrm{m} / \mathrm{s})$ [13].

Young et al. [44] obtained the expression for droplet's velocity

$$
U=\frac{a}{3 \eta_{i}+2 \eta_{o}}\left(-\frac{\partial \gamma}{\partial C}\right) \nabla C_{\infty},
$$

where $C_{\infty}$ is the undisturbed solute concentration, $a$ is the radius, $\eta_{i}$ and $\eta_{o}$ are viscosities of inner and outer fluids. For the case of a solid particle $\left(\eta_{o} \rightarrow \infty\right)$, the velocity calculated by eqn.(1.6) goes to zero. However, early experiments in the 80s [45-49] have shown that concentration gradients of solutes are able to propel solid particles. A fact predicted by Derjaguin et al. in 1947 [50]: the diffuse nature of the interface enables the formation of osmotic flows due to a gradient field e.g. chemical concentration or temperature. This is a consequence of a mechanism, known as phoretic effect [43], that generates slip velocity at the interface due to interactions with the surface of the particle.

The mechanism of flow generation at the fluid-fluid interface is called the Marangoni 
effect. Due to an interfacial tension gradient at the interface, a shear stress jump is induced which results in velocity continuity and stress discontinuity at the interface [43, $51,52]$. In contrast, in the phoretic effect, a local gradient can induce a slip velocity at the (typically fluid-solid) interface [43].

At the interfacial region, with thickness $\zeta$, the particle phase and the outer fluid phase coexist and interact [53]. While at the particle scale, the velocity and/or the stress fields are discontinuous, at the interfacial layer, they are continuous and determined through the interactions between the two phases. Typically, there is an order-of-magnitude difference between the particle radius and the interfacial layer thickness $(a \gg \zeta)$. Thus, we can take advantage of the separation of length scales. One can treat the interfacial layer as a virtually flat surface, and solve it for velocity and stress profiles. Then, use those values as boundary conditions at the interfaceassuming $\zeta \approx 0$ - to solve the problem at the particle scale.

To understand the physico-chemical interactions at the interfacial layer, one needs to decipher the interplay of fluid dynamics, interfacial dynamics, and transport of mass, charge, or thermal energy. Depending on the type of involved field, the particle can undergo propulsion due to a gradient in an electric field (electrophoresis), temperature (thermophoresis), or chemical concentration field (diffusiophoresis). The specific details for modelling each case can be found in reference [43].

To describe the general case for the motion of an autophoretic particle driven by diffusiophoresis and Marangoni effects, we consider a force-free spherical droplet of radius $a$ and overview the involved equations and associated boundary conditions following Morozov and Michelin's work [54] that modifies the Anderson model [43] for an active droplet. The first assumption is that the capillary number $C a=\eta_{o} U / \gamma_{0}$ is small, i.e. capillary pressure dominates drag preventing the droplet from deformations due to hydrodynamic forces. For typical values of radius $a$ and propulsion velocity $U$, $R e=\frac{\rho U a}{\eta_{o}} \ll 1$. Thus, one can use Stokes equation for both outer phase (swimming medium) and inner phase (droplet).

$$
\begin{aligned}
& -\nabla p_{o}+\eta_{o} \nabla^{2} \mathbf{u}_{o}=0, \quad \nabla \cdot \mathbf{u}_{o}=0 \\
& -\nabla p_{i}+\eta_{i} \nabla^{2} \mathbf{u}_{i}=0, \quad \nabla \cdot \mathbf{u}_{i}=0
\end{aligned}
$$

where $o$ and $i$ respectively denote outer and inner phase. With the assumption that there is no transport of solute into the droplet, the concentration of solute molecules 
$C$ in the outer phase can be found by solving the advection-diffusion equation:

$$
\frac{\partial C}{\partial t}+\mathbf{u}_{o} \cdot \nabla C=\mathcal{D} \nabla^{2} C
$$

where $\mathcal{D}$ is the diffusion coefficient of the solute. After knowing the governing equations, one needs the proper boundary conditions to solve the system of equations.

The solute interacts with the interface via a chemical reaction. For the sake of simplicity we assume a constant reaction rate $\mathcal{A}$ at the interface.

$$
\mathcal{D} \mathbf{n} \cdot \nabla C=\mathcal{A} \quad \text { at } r=a,
$$

where $\mathbf{n}$ is the outward normal to the interface.

The diffusiophoretic effect causes a finite slip velocity at the interface

$$
\mathbf{u}_{i}-\mathbf{u}_{o}=\mathcal{M}(\mathbf{I}-\mathbf{n n}) \cdot \nabla C \quad \text { at } r=a .
$$

where $\mathcal{M}$ is the phoretic mobility coefficient and $\mathbf{I}$ is the identity tensor.

To account for the Marangoni effect we consider a stress jump at the interface. For simplicity, we do a first-order approximation that interfacial tension changes linearly with the solute concentration at the interface

$$
\gamma=\gamma_{0}-\gamma_{C}\left(C-C_{\infty}+\frac{\mathcal{A} a}{\mathcal{D}}\right) \quad \text { at } r=a .
$$

Here, $\gamma_{0}$ is the reference value of interfacial tension at a solute concentration of $C=C_{\infty}-\mathcal{A} a / \mathcal{D}$ and $\gamma_{C}$ is a positive constant. The gradient in interfacial tension contributes to the balance of stresses. For a non-deformable droplet the balance of tangential stress at the interface results

$$
\mathbf{n} \cdot\left(\boldsymbol{\sigma}_{i}-\boldsymbol{\sigma}_{o}\right)=-\gamma_{C}(\mathbf{I}-\mathbf{n n}) \nabla C \quad \text { at } r=a
$$

where $\boldsymbol{\sigma}$ is the stress tensor of the fluids. At regions far from the swimmer, the flow velocity in the swimmer reference frame and the solute concentration have the fixed values of

$$
\mathbf{u}_{o}=U_{\infty} \boldsymbol{e}_{z} \text { and } C=C_{\infty}
$$

Anderson [43] obtained a general formula for droplet's velocity which included the effects of stress jump and velocity jump at the interface. A modified version of it 
which is the terminal velocity of a droplet driven by a solute gradient of $\mathcal{A} / \mathcal{D}$ due to a combination of diffusiophoresis and Marangoni effects is

$$
\mathcal{V}=\frac{\mathcal{A}\left(\gamma_{C} a+3 \eta_{i} \mathcal{M}\right)}{\mathcal{D}\left(2 \eta_{o}+3 \eta_{i}\right)}
$$

\subsubsection{Mechanisms for symmetry breaking}

So far, it has been shown that a fore-aft asymmetry in a field is necessary to propel a phoretic particle. However, an isotropic particle, which undergoes isotropic surface activity, generates a homogeneous concentration field, and thus will not self-propel [10]. Hence, the symmetry of the system should be somehow broken. For a particle driven by Marangoni and diffusiophoresis, the solute concentration field is coupled to the hydrodynamic field via the advection-diffusion equation (eqn.(1.9)). We can use the terminal velocity $\mathcal{V}$ to define a Peclet number $P e=\mathcal{V} a / \mathcal{D}$ which characterizes the relative strength of advective transport to diffusive transport for the solute. Using $P e$, one can non-dimensionalize eqn.(1.9):

$$
\frac{\partial c}{\partial t}+\mathbf{u} \cdot \nabla c=\frac{1}{P e} \nabla^{2} c
$$

For the diffusive regime (the limit of $P e \rightarrow 0$ ), the solute concentration field can be described by the steady state diffusion equation

$$
\nabla^{2} c=0
$$

which is linear. With negligible advection, the coupling between solute transport and fluid dynamics - through $\mathbf{u} \cdot \nabla c$ term- disappears. The diffusion and the Stokes equations are linear and cannot result in an instability. Hence, the asymmetry should be imposed geometrically. On the other hand, when $P e$ has a finite value, solute transport and fluid dynamics are coupled and the nonlinear advective term $\mathbf{u} \cdot \nabla c$ can be a source for instabilities and spontaneous symmetry breaking $[10,19,53]$.

\subsubsection{Intrinsic symmetry breaking}

Intrinsic symmetry breaking means the asymmetry is imposed on the systems, typically geometrically, and is not a result of dynamical instability. Thus, it is useful for the diffusive regime where $P e \ll 1$. The broadly-adopted approach for creating such an asymmetry is to spatially vary the surface activity. Janus particles are well-known 
examples of this approach. They can be spherical, rod-shaped, porous, etc. They are usually made by coating one half of a silica micro-particle with a reactive metallic material [55]. This leads to asymmetric reaction rate and, in turn, asymmetric solute distribution resulting in a slip velocity.

The isotropic concentration field can be distorted by adding a secondary particle of a different size. In this two-sphere system, both particles have isotropic surface activity and mobility, and the symmetry is broken geometrically [56].

\subsubsection{Spontaneous symmetry breaking}

As discussed before, a particle with isotropic surface activity can undergo a dynamical instability that leads to self-propulsion. This instability originates from the complex coupling between hydrodynamic and chemical fields through the nonlinear advective term $\mathbf{u} \cdot \nabla c$ in the advection-diffusion equation. It has been shown theoretically that for an active isotropic particle, the motionless solution becomes unstable when $P e>4$. In this regime, the stable solution is a particle that moves with a finite velocity (Fig. 1.4) due to a concentration field with a dipolar, fore-aft asymmetry [57]. Same author, in reference [58], overviews different scenarios for an active droplet which emits solute (summarized in Fig. 1.5).

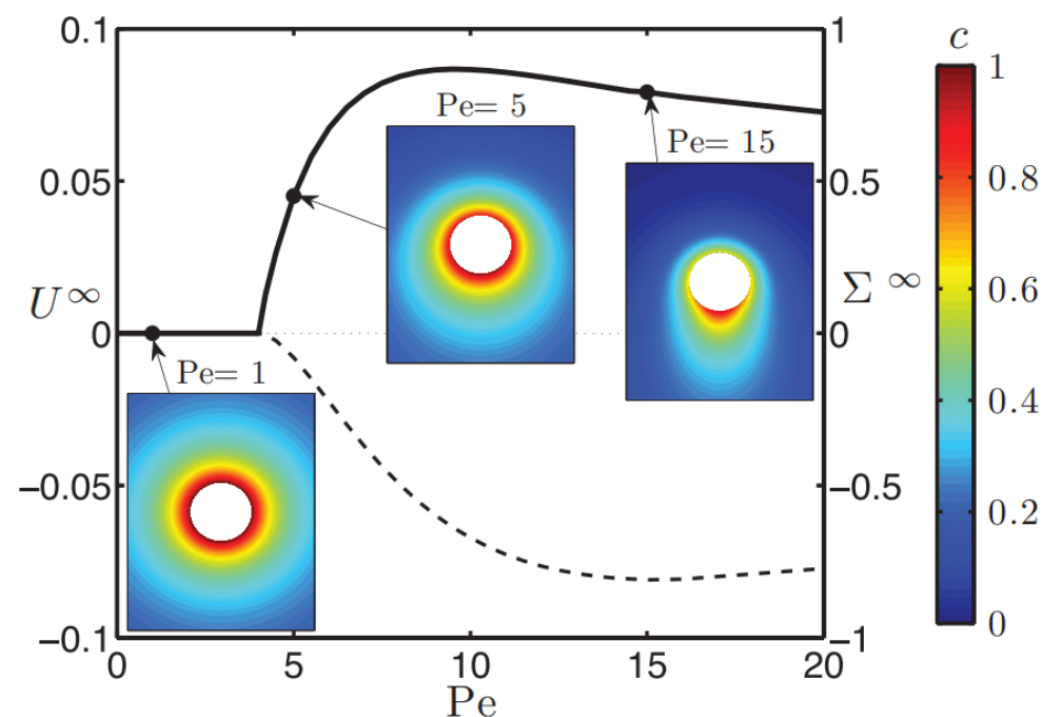

Figure 1.4 - Terminal swimming velocity of an autophoretic particle, $U_{\infty}$ (solid line) as a function of the Peclet number. The steady state solute distribution around the particle is plotted for $P e=1, P e=5$, and $P e=15$. The swimmer is swimming upwards. Taken From [57] with permissions. 


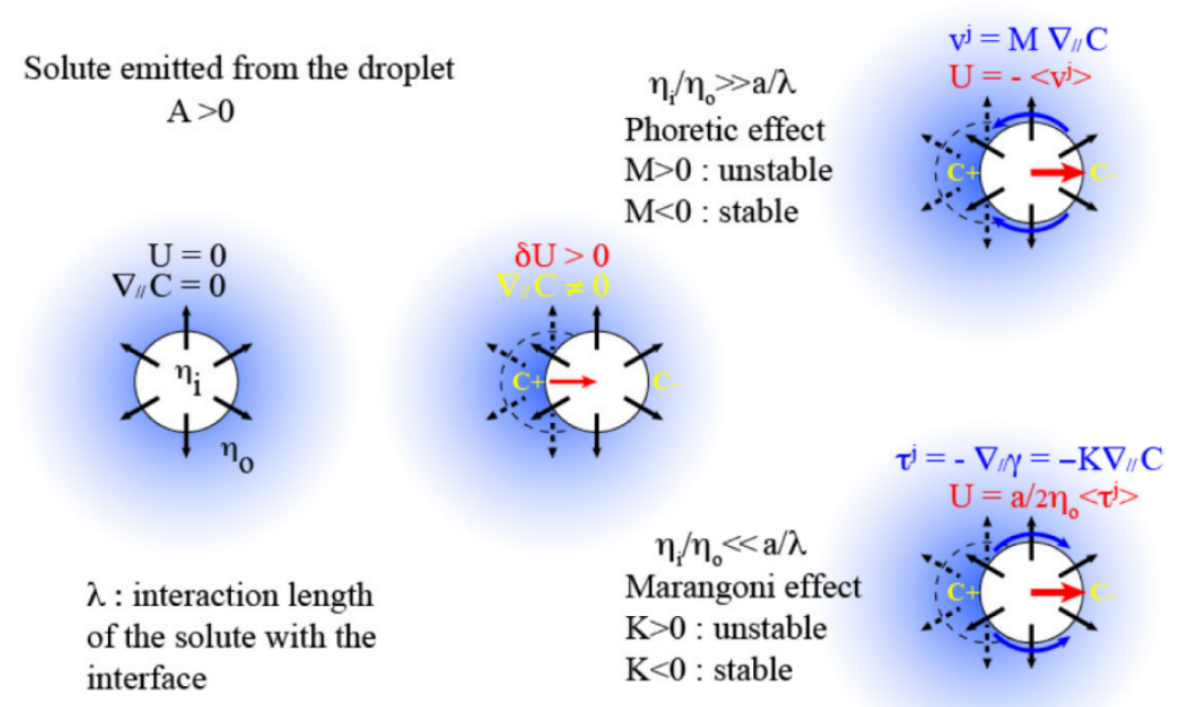

Figure 1.5 - The spontaneous symmetry breaking and self-propulsion mechanism for an isotropic droplet. Taken From [58] with permissions.

\subsubsection{Active droplets}

The framework discussed in Section 1.3.1 is applicable to any autophoretic particle which undergoes chemical activity at its surface such as active droplets. Active droplets, in particular, are known as the experimental realization of isotropic autophoretic particles. Estimates from scaling analysis suggest that the dominant driving force for active droplets is provided by Marangoni stresses (see Chapter 3). Thus, active droplets have to induce a self-sustaining fore-aft gradient in interfacial tension to achieve continuous propulsion. This gradient generally originates from a chemical activity at the interface which drives a fluid dynamical advection-diffusion instability. Based on the way the energy for activity is obtained, the experimental systems fall into three distinct categories: i) a chemical reaction taking place inside the droplet, ii) phase separation, and iii) micellar solubilization.

\section{i. Internal chemical reaction}

Since the chemical reaction takes place inside the droplet, this type of active droplet, compared to its counterparts, has a shorter lifetime and thereby shorter cruising range, i.e. the maximum distance the swimmer can self-propel before stopping. The mechanism depends on a specific chemical reaction, therefore, it is challenging to tune, modify, or improve. This limits the applications as well as further studies where for 
example one needs to change the composition of the environment.

In 2006, Toyota et al. [59] observed self-propulsion for octyloxybenzaldehyde droplets immersed inside an aqueous solution of a reactive surfactant (10-(4-aminophenoxy)decyltrimethylammonium bromide). They reported internal convection and formation of vesicles at the wake of the droplets (Fig. 1.6 a,b). They showed that the vesicles were created by molecules that were a product of a condensation reaction between the amino group of the surfactant molecules and the formyl group of the droplet molecules. Another example for these systems is the application of a spatiotemporal chemical oscillator as a fuel source, known as Belouzov-Zhabotinsky (BZ) reaction [60-62]. The majority of the experimental systems however work based on chemical reactions happening for a surfactant layer at the droplet interface. In an oil-in-water system, the droplet is loaded with a surfactant precursor. The precursor undergoes hydrolysis at the interface and generates the surfactant [63] (Fig. 1.6c). The advection-driven instability then results in the sustained interfacial tension gradient which propels the droplet. Ban et al. [64] studied the self-propulsion of nitrobenzene droplets inside an aqueous solution, reporting the controlled droplet swimming via tuning the $\mathrm{pH}$ value of the environment. The droplets contained di(2-ethylhexyl)phosphoric acid (DEHPA), which exhibits intermediate surface activity when protonated. The deprotonation of DEHPA was the reaction rendering the molecules more surface active in comparison with the pristine molecules. The reaction rate was a function of the $\mathrm{pH}$ value of the aqueous phase.

Thutupalli et al. developed a system where a water droplet, loaded with bromine, self-propels in an oil phase which includes monoolein as a surfactant. The bromination of the surfactant increases the interfacial energy at the leading front of the droplet [65] (Fig. 1.6 d).

\section{ii. Phase separation}

Spontaneous symmetry breaking and subsequent instabilities can also develop in ternary liquid solutions, provided that the composition alters, crossing the two-phase line in the phase diagram. This phase separation can happen when components evaporate at different rates into the bulk medium, or when a substance is adsorbed from the bulk into the droplet. Experimental realizations for such systems include a mixture of water, ethanol, surfactant in oil [68], and solutions of polymers and salts undergoing phase separation $[69,70]$. 


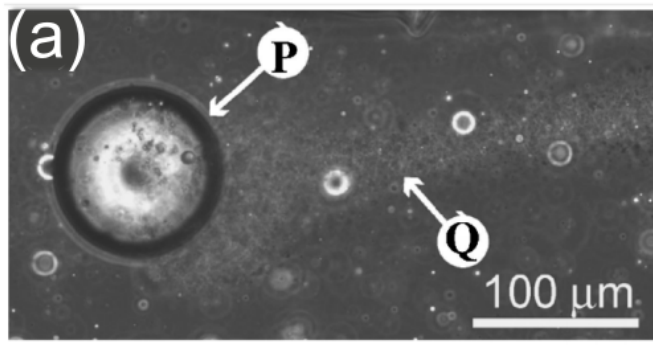

(b)
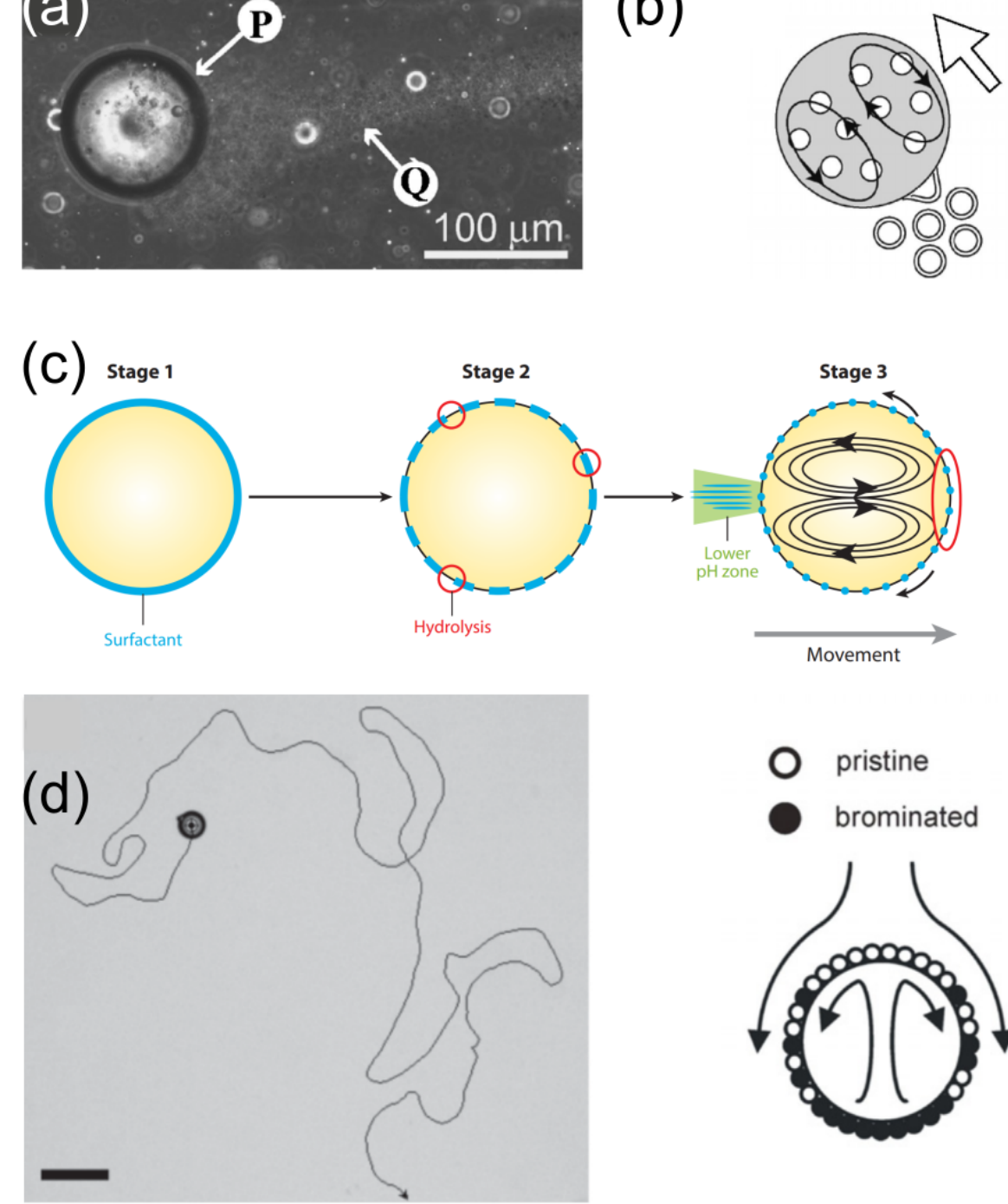

O pristine

brominated

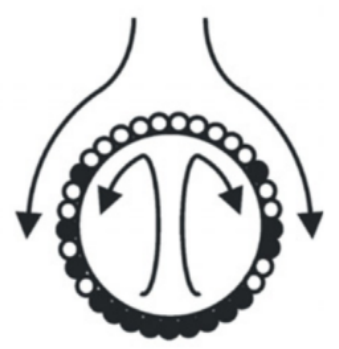

Figure 1.6 - (a) Phase-contrast images of swimming oil droplet (site P) with a trail of giant vesicles (site $Q$ ) and (b) the schematic of propulsion mechanism from [66]. (c) Onset of symmetry breaking leading to propulsion of an oil droplet in an aqueous surfactant solution in Reference [63]. From [67] with permissions. (d) The trajectory of a water droplet, loaded with bromine, which self-propels in an oil phase containing monoolein as surfactant, from [65] with permissions. 


\section{iii. Micellar solubilization}

Surfactant-stabilized droplets inside a micellar solution can also spontaneously selfpropel. Unlike previous examples, in this system, the gradient in surfactant coverage at the droplet interface is produced not by a chemical reaction but by an inhomogeneous depletion driven by a solubilization process [67]. This process is known as micellar solubilization [71]. When an oil droplet is placed in an aqueous surfactant solution at a concentration well above the critical micelle concentration (CMC), it starts to solubilize: oil molecules move from the interface into the surfactant micelles in the aqueous solution. Hence, the final state is a homogeneous suspension of oil-filled micelles i.e. a nanoemulsion. If the surfactant concentration is high enough, the droplet displays a spontaneous symmetry breaking and starts to self-propel.

Micellar pathway

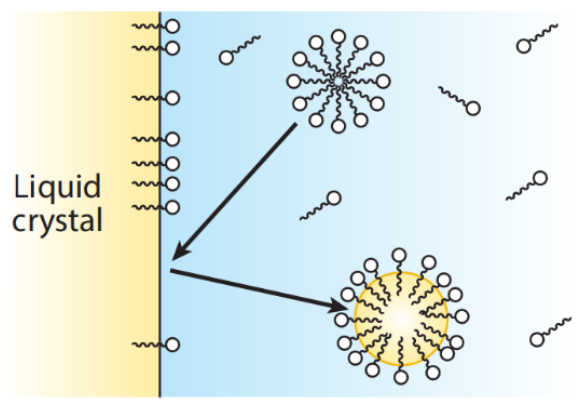

Molecular pathway

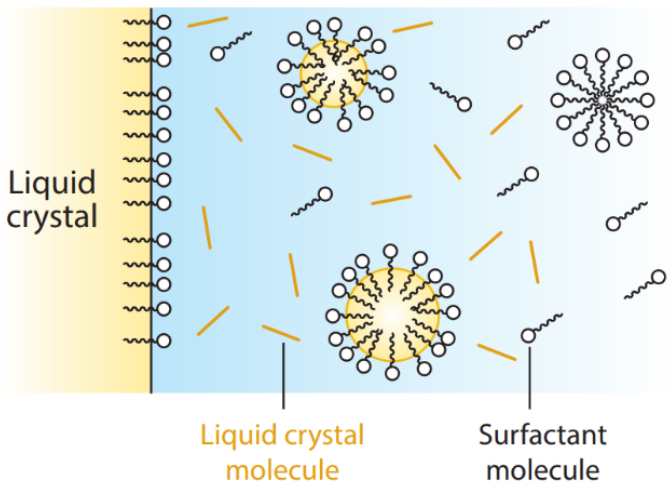

Figure 1.7 - Schematic illustration of the micellar and molecular pathways for the solubilization of an oil droplet inside a micellar surfactant solution. The oil phase is denoted by $L C$ since in that particular study it is $5 C B$ which is a nematic liquid crystal. In the micellar pathway, micelles collect oil through impinging directly onto the droplet interface. In the molecular pathway, the oil molecules diffuse into the aqueous phase and micelles collect them and grow. From [67] with permissions.

There are hypotheses to describe the mechanism of solubilization. Consider an oil droplet in an aqueous micellar solution (the reversed case is also possible). The oil droplet is covered with a surfactant monolayer. There are two possible, competing pathways for solubilization [71, 72]: i) micellar pathway, during which micelles collect oil molecules through direct impinging onto the interface, or ii) molecular pathway, where micelles take up oil molecules that have diffused from the droplet into the aqueous solution [67] (see also Fig. 1.7). The solubility of the oil phase in water and the potential electrostatic repulsion between micelles and the surfactant monolayer at 
the interface dictates which pathway is dominant. For systems with an ionic surfactant, the latter is particularly important.

Since during the solubilization process, the oil-filled micelles grow in size, they require more molecules - compared to empty micelles - in their surfactant monolayer. Thus, the surfactant concentration at the vicinity of the interface is depleted. This deficit of surfactant molecules is compensated by taking molecules either directly from the interface (micellar pathway) or from the background of free surfactant molecules in equilibrium with the interface (molecular pathway) [67]. Fig. 1.8) illustrates the respective concentration profiles of the oil and surfactant molecules as well as the empty micelles. Solubilizing droplets outperform other systems in terms of activity duration-in the order of hours- and thereby cruising range.
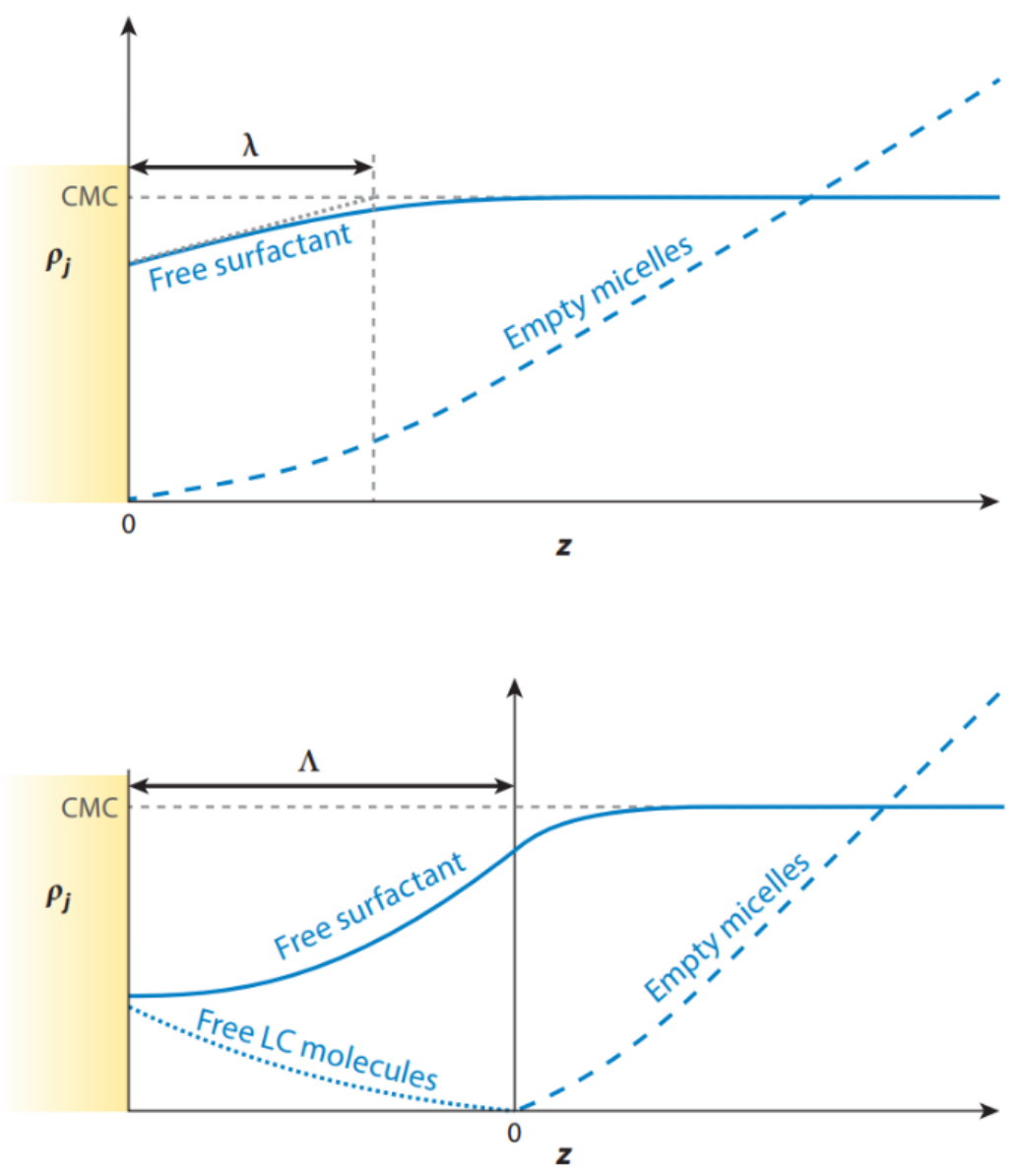

Figure 1.8 - Depiction of the concentration profiles of the free surfactant molecules, diffused oil (LC) molecules, and empty micelles. $\rho_{j}$ is the number density of a particular species, and the $z$ denotes the distance from the droplet interface (located at $z=0 \mathrm{in}$ (a) and at some value $z<0$ in (b)). (a) Micellar pathway and (b) Molecular pathway. From [67] with permissions. 
The surfactant coverage at the interface is coupled to the background concentration field at the vicinity of the interface. Thus, the interfacial tension rises with the local ratio of filled to empty micelles. When experiencing an advective perturbation, the radial symmetry of the micelle distribution around the droplet is spontaneously broken generating a front-aft asymmetry. This asymmetry results in an interfacial tension gradient and thereby Marangoni stresses which drive the droplet forward Section 4.3. The details of the advection-diffusion model that predicts the interfacial instability can be found in $[13,54,73]$.

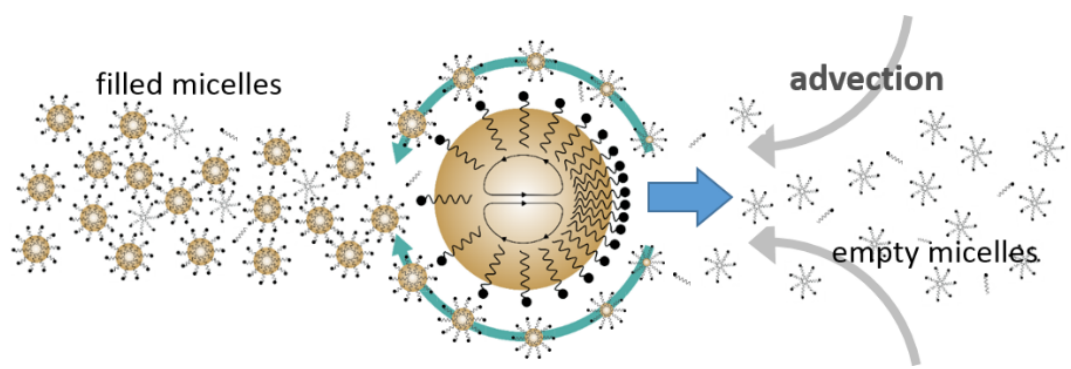

Figure 1.9 - The schematic illustration of the Marangoni-driven propulsion mechanism in solubilizing droplets.

\subsection{Collective dynamics in active matter}

The non-equilibrium nature of active matter can result in pattern formation behavior. Due to interactions of each constituent with other counterparts and with the environment, the fluctuations at the microscopic scale can get amplified and result in dynamical instabilities [10, 74]. Consequently, macroscopic coherent structures can emerge out of these mechanical instabilities. The spontaneous emergence of order due to local interactions between the constituents of an initially disordered system is known as self-organization or self-assembly. It requires a constant source of energy to overcome the propensity of the system to move towards disorder. In active matter, this energy is injected at an individual level [75].

Two ubiquitous features of collective dynamics in the active matter are phase separation and collective directed motion [10]. An example of the first one is motility-induced phase separation (MIPS). For instance, a suspension of active colloids can undergo a crystallization process that is driven by inter-particle attractive chemical interactions [76-78] (Fig. 1.10). Examples for the second one can be found in bacterial colonies such as suspensions of Bacillus subtilis exhibiting bacterial turbulence (Fig. 1.11a), 
algal suspensions like Euglena gracilis forming bio-convection patterns (Fig. 1.11 b), all the way to larger scales such as fish schools and bird flocks (Fig. 1.11c). In these systems, directional ordering results in long-range correlations in the movement of the neighboring constituents.

As discussed before, autophoretic colloids and droplets are chemically active. They locally generate a chemical gradient at their surface that induces a flow which in turn sets the surrounding fluid into motion. Depending on the relative inter-particle distance, the particles can interact chemically- by changing the concentration fields of one another- or hydrodynamically- through mutual entrainment. Such chemohydrodynamic interactions can result in states of nontrivial collective behavior [79-81]. For details, see [10]. In the following, we discuss different types of interactions between phoretic particles.

\subsubsection{Collective dynamics governed by short-range interac- tions}

For systems in equilibrium, the steady state is independent of the kinetic parameters. On the contrary, in a crowded suspension, the local concentration of active particles is higher at regions where their motility is slower [9]. They tend to slow down at high-density regions in the domain where short-range steric interactions between particles slow them down. This positive feedback loop results in motility induced phase separation between dense and dilute phases of the suspension. Buttinoni et al. [76] experimentally showed the dynamical cluster formation of active Janus particles governed mainly by steric interactions.

\subsubsection{Collective dynamics governed by long-range interactions}

Long-range inter-particle interactions in phoretic suspensions include [10]

(i) Chemical interactions. The chemical activity of these particles generates local gradients in the chemical species concentration field and in turn, influences the dynamics of the neighboring particles.

(ii) Hydrodynamic interactions. The flow generated by one moving particle can influence the dynamics of other counterparts and vice versa.

The hydrodynamic field induced by particles depends on the chemical field gradients and similarly, the flow of particles can alter the chemical field. Hence, these two fields are dynamically coupled, and the collective dynamics are dictated by their interplay. 

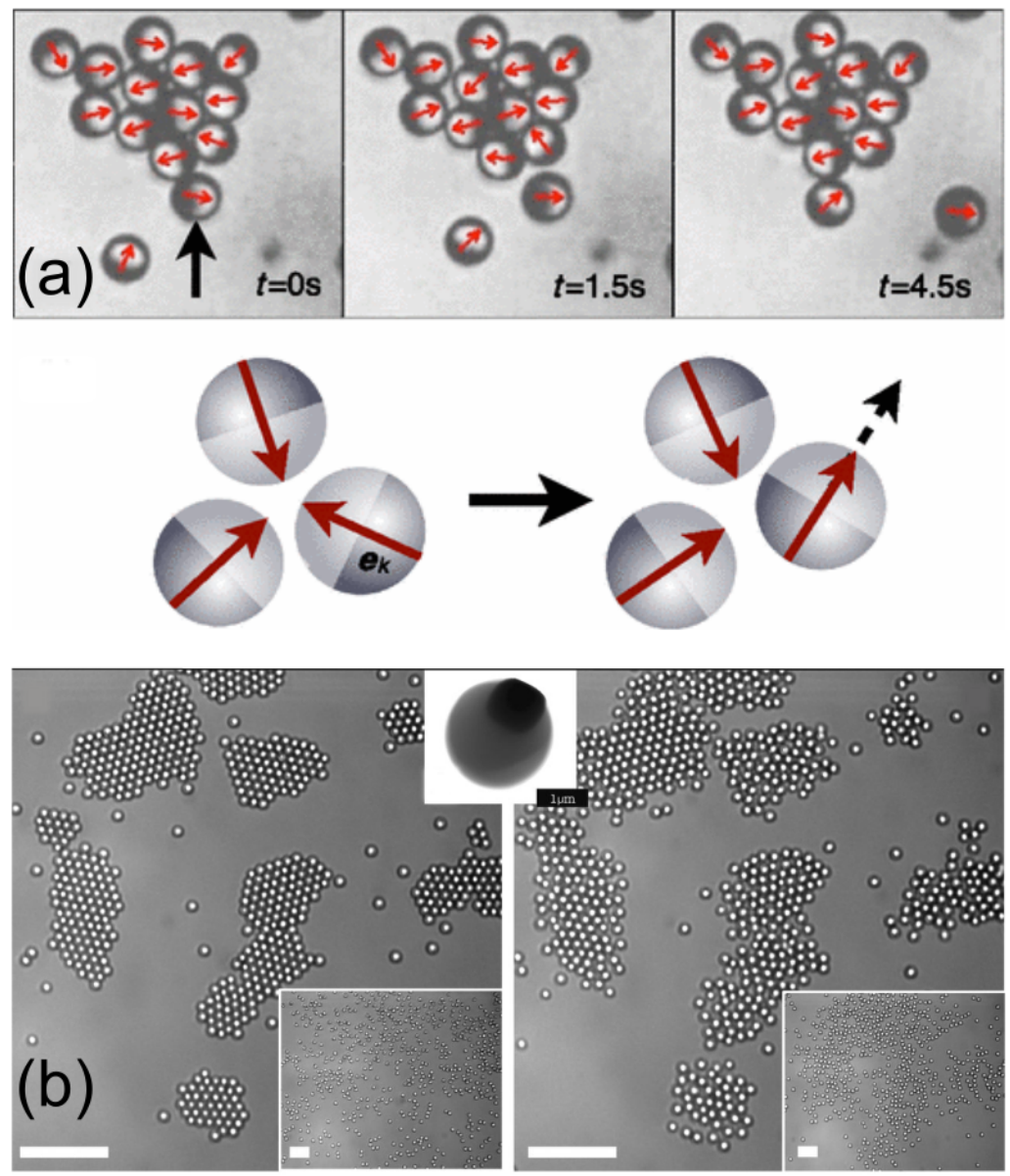

Figure 1.10 - Motility induced phase separation in suspensions of synthetic active particles. (a) Snapshots of a cluster of active carbon-coated Janus particles. Taken from [76], (b) Left: living crystals of photo-activated colloidal particles form from a homogeneous suspension (inset) when illuminated. Right: The crystals melt by thermal diffusion when light is turned off: the snapshot shows the system after $10 \mathrm{~s}$ (inset, after $100 \mathrm{~s})$. Reprinted from [78].

\subsubsection{Chemical interactions}

The phoretic particles move towards or away from concentration gradients depending on the type of chemical reaction. Assume a point source of a chemical species undergoing diffusion in the surrounding medium. It will generate a steady-state concentration field that decays as $\sim 1 / r$. Where $r$ is the distance from the source. A phoretic particle influenced by this gradient will experience a chemotactic drift velocity that is proportional to the strength of the gradient and consequently, decay as $\sim 1 / r^{2}$. Such long-range interactions, if attractive, can give rise to cluster formation even in dilute systems [84-86]. Driven by these interactions, Janus particles have shown clustering 
formation at a packing fraction smaller than $10 \%[84,85,87]$. In the absence of attractive chemical interactions, that number rises to more than 30\% [88]. Depending on the diffusive time scale of the chemical species, and the nature of the interactions, further non-trivial collective behavior might also emerge. Self-propelling droplets undergoing micellar solubilization have shown negative (repulsive) autochemotactic behavior in response to slowly-diffusing chemical trails of their counterparts [89]. While the trailmediated interactions in biological swimmers have been studied at individual [90] and collective [91] scales, the autochemotactic interactions and subsequently, collective dynamics in such active droplets is virtually unexplored.

\subsubsection{Hydrodynamic interactions}

The swimming medium plays a crucial role in inter-particle interactions. Microorganisms usually swim in semi-dilute or dense suspensions. The velocity field generated by a force-free swimmer, in the absence of boundaries, decays as $\sim 1 / r^{2}$ [30]. Once a swimmer is close enough to feel the flow field of a neighbor, it will (i) be carried away (entrained) by that flow and (ii) change its orientation in response to the gradients in the velocity field generated by the neighbor. In dense suspensions, these interactions can influence the dynamics at the entire population scale. For example, dense bacterial suspensions, show large-scale coherent flow patterns in the form of swirls and jets [30, 92-98] and Wood mouse spermatozoa swim faster by forming aggregates [99]. Two-dimensional simulations of active particles, taking into account the near-field hydrodynamic effects, have shown cluster formation. For active droplets, it has been shown that in the presence of a boundary (typically the bottom surface of a reservoir) swimmers attract each other and form multiple two-dimensional clusters that hover over the bottom surface [79]. It is indeed an example of flow-induced phase separation [80].

\subsection{Scope of this Thesis}

In this thesis, we aim to propose active droplets as a minimal paradigm and a testing bed to investigate a number of current problems in biomimetic swimmers and active matter. Previous works on active droplets have shown some features of the system that mimic the hallmarks of life. However, there is still a long way ahead. To develop a lifelike protocell, we have to provide routes for functionality beyond mere self-propulsion. 
In the framework of a droplet swimmer as a protocell, there are nontrivial challenges to be addressed:

Energy transduction. In Section 1.3.3, we discussed the possible route to use chemical reactions as a source of energy for activity-such as motility. Such selfsustaining reactions maintain the out-of-equilibrium state of the system.

Compartmentalization. While adding internal compartments to the protocell is critical in the context of biomimicry, from an applied science perspective, active droplets can be used as cargo carriers, sensors, or micro-reactors. In both cases, we need to incorporate internal structures that are stable and do not hamper the droplet motility.

Adaptability. As summarized in section 1.3.3, there are multiple studies that report motility for active droplets. However, due to the complex coupling between the fluid flow and chemical transport, there exist many questions about how a droplet would react to changes to its environment. Namely, response to changes in the viscosity, interactions with boundaries, and adapting their dynamics when interacting with their counterparts.

Information processing. Active droplets possess the capability to sense and respond to signals from the environment. Particularly, they have been shown to undergo chemotaxis as well as autochemotaxis [89]. However, we still lack the quantitative understanding of the autochemotactic interactions between droplets and the consequences within active emulsions.

Growth and division. Since droplets are not solid, they have the potential to deform and even divide. Growth and division through phase separation have been reported theoretically for droplets [100].

In this thesis, we address some of these challenges, aiming for a general experimental framework that is applicable to a broad range of autophoretic particles. In terms of fabrication, we have adapted and improved microfluidic techniques to generate compartmentalized droplets. To elucidate the complex interplay of hydrodynamics and transport of chemical species, we have introduced a visualization technique that significantly advances the state of the art of time-resolved imaging these fields. Based on our studies on the individual particle scale, we show first results on observing and analyzing the more complex emergent dynamics of collective systems. 


\subsection{Structure of the thesis}

The remainder of this thesis, including 4 publications and preprints, is structured as follows:

In Chapter 2 [101], we address the compartmentalization challenge in active droplets. We use microfluidics to incorporate an aqueous core inside a liquid crystal droplet; a composite design known as a double emulsion or a liquid shell. While inactive double emulsions are stable against bursting, the internal convective flow in motile shells can displace the core toward the interface and cause coalescence. We show how the nematoelasticity of the shell phase impedes this the coalescence, resulting in a stable motile liquid crystal shell. We further study the propulsion dynamics of the shells rationalizing a peculiar shark-fin like trajectory in 2D and a helical trajectory in 3D.

In Chapter 3 [102], we study how an active droplet adapts to the variation of viscosity of the swimming medium. We report that, as a response to increasing the viscosity, droplet dynamics transition from steady propulsion to bimodal chaotic swimming. To elucidate the non-linear coupling between the hydrodynamics and chemical fields, we developed a novel technique to simultaneously visualize the two fields around the droplet.

Chapter 4 [103] presents a brief review of studies on chemotactic interactions in our group, followed by experimental results that investigate complex interactions of droplets with external and self-generated chemical fields and complex environments, demonstrated by the peculiar interactions of droplets with microfluidic pillar arrays. This motivated us to go for a full quantitative study of the elementary case of two droplets interacting via their chemical signatures in a 2D space.

This quantitative analysis is presented in Chapter 5 [104]. We derive a phase diagram for the autochemotactic interactions in two dimensions, sorted by trail-crossing and reflecting interactions. In the second part of the study, we investigate the collective dynamics, governed by autochemotactic interactions, in 2D and 3D. We report a novel state of dynamical arrest in active emulsions: 'autochemotactic caging', where swimmers are temporarily trapped in an evolving network of repulsive chemical trails.

Chapter 6 presents our work on the emergence of spontaneously-rotating clusters of active droplets. We explain the origins of rotation for single isotropic droplets and extend our understanding to these clusters that have formed due to hydrodynamic interactions. 
Finally, Chapter 7 summarizes and contextualizes the findings and implications of the previous chapters, highlighting active droplets as a promising model for autophoretic active matter. We present a brief summary of ideas for continuing projects based on the studies outlined above, some of which have already been initialized. 

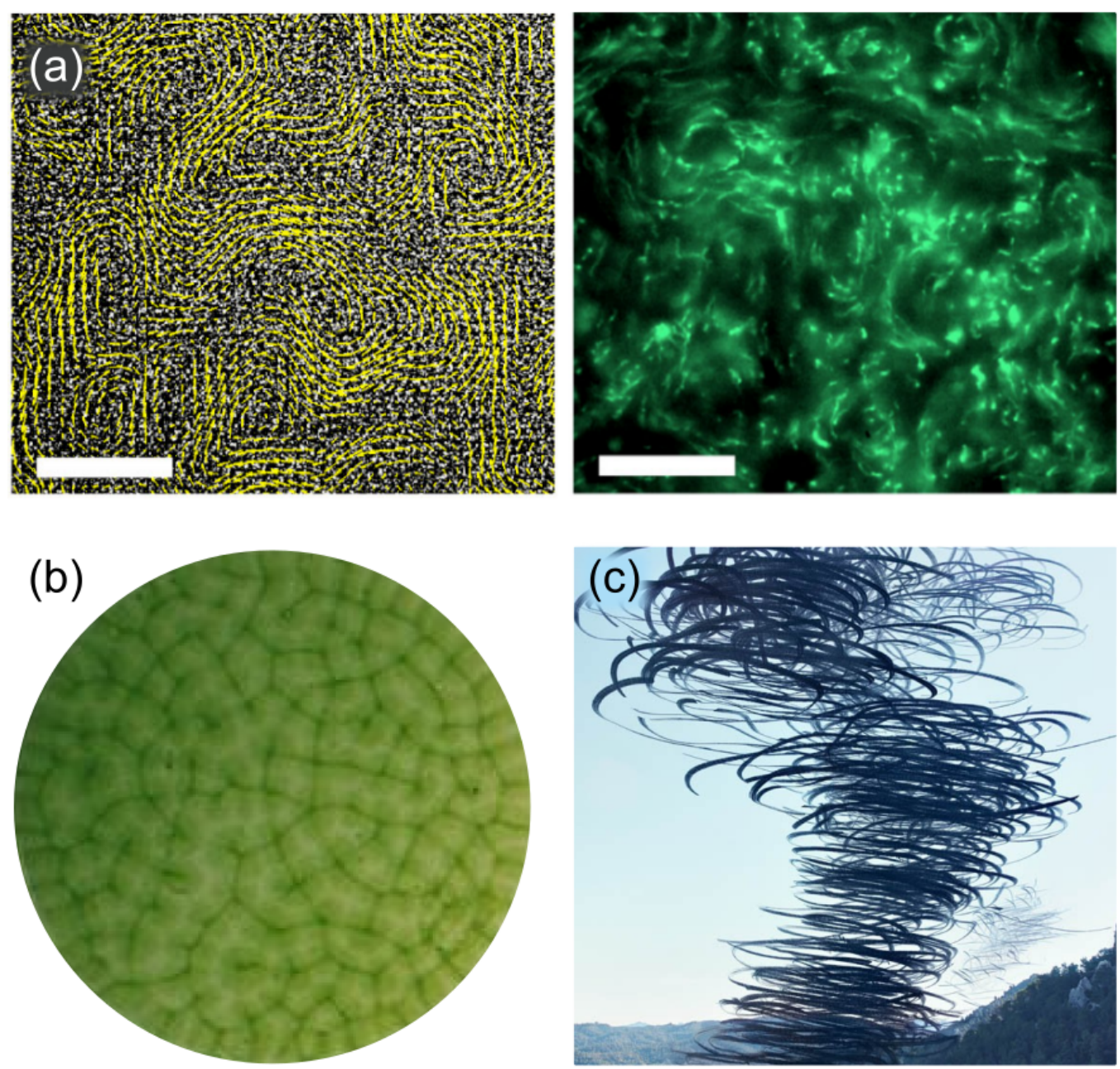

Figure 1.11 - Examples of collective directed motion in nature. (a) Bacterial turbulence within a dense suspension of Bacillus subtilis; yellow lines show the flow field of bacterial migration. Taken from [4], (b) Bioconvection pattern inside a suspension of Euglena gracilis. Reprinted from [82], (c) Trajectories of birds flying in a flock. The Photo is the courtesy of Xavi Bou. Taken from [83]. 



\title{
Chapter 2
}

\section{Topological stabilization and dynamics of self-propelling nematic shells ${ }^{1}$}

\begin{abstract}
Liquid shells (e.g. double emulsions, vesicles etc.) are susceptible to interfacial instability and rupturing when driven out of mechanical equilibrium. This poses a significant challenge for the design of liquid shell based micro-machines, where the goal is to maintain stability and dynamical control in combination with motility. Here, we present our solution to this problem with controllable self-propelling liquid shells, which we have stabilized using the soft topological constraints imposed by a nematogen oil. We demonstrate, through experiments and simulations, that anisotropic elasticity can counterbalance the destabilizing effect of viscous drag induced by shell motility, and inhibit rupturing. We analyze their propulsion dynamics, and identify a peculiar meandering behavior driven by a combination of topological and chemical spontaneously broken symmetries. Based on our understanding of these symmetry breaking mechanisms, we provide routes to control shell motion via topology, chemical signaling and hydrodynamic interactions.
\end{abstract}

\footnotetext{
${ }^{1}$ This chapter is published in Hokmabad, B. V., Baldwin, K. A., Krüger, C., Bahr, C., \& Maass, C. C. (2019). Topological stabilization and dynamics of self-propelling nematic shells. Physical review letters, 123(17), 178003. B. V. H. designed the research, performed experiments and data analyses and wrote the manuscript. K. A. B. performed experiments and data analyses and wrote the manuscript. C. K. designed the research and performed experiments. C. B. performed the numerical simulation and wrote the manuscript. C. C. M. designed the research, performed data analyses and wrote the manuscript.
} 


\section{$2.1 \quad$ Introduction}

The capability to produce controllable, actively self-propelling microcapsules would present a leap forward in the development of artificial cells, microreactors, and microsensors. Inactive microcapsules have been developed in the form of double emulsions (droplet shells), which have been applied as, e.g., reactive microcontainers [105], synthetic cell membranes [106], food and drug capsules [107, 108], optical devices [109-111], and biotic sensors [112]. However, these highly structured compound droplets are usually non-motile, and any actuation that displaces their liquid cores makes them susceptible to shell rupture if the interfaces of the nested compartments can coalesce. Alternative compartmentalized structures such as vesicles, capsids or polymersomes typically possess immobile interfaces which impede self-actuation. Hence, engineering such motile systems requires further complexities in design and fabrication [113-115]. In contrast, to survive motility, any liquid shell with mobile interfaces requires a stabilizing force to counter the destabilizing swimming dynamics.

In this paper, we present a new approach to the problem of combining encapsulation with autonomous motility, by using nematic active double emulsions, where anisotropic micellar solubilization induces motility, and the nemato-elasticity of the shell provides stability without requiring further complexities in the design. Through experiments and simulation of the elastic energy in the liquid crystal shell, we show that active shells are stable only in the nematic state. We demonstrate that the shell dynamics are dictated by anisotropic self-generated chemical fields, broken topological symmetries, and hydrodynamic interactions, and that by tuning these factors we can control and direct their motion, providing avenues for applications in transport, guidance and targeted release. Our framework provides a bottom-up approach for developing functional micro-machines using established physicochemical mechanisms.

\subsection{Results and discussion}

Our active double emulsion system is comprised of water-in-oil-in-water droplet shells. Shells self-propel while slowly dissolving in a micellar surfactant solution. Micelles swell while filling with oil, which depletes the surfactant coverage of the shell's posterior. This induces a self-sustaining tension gradient in the external oil-water interface that 


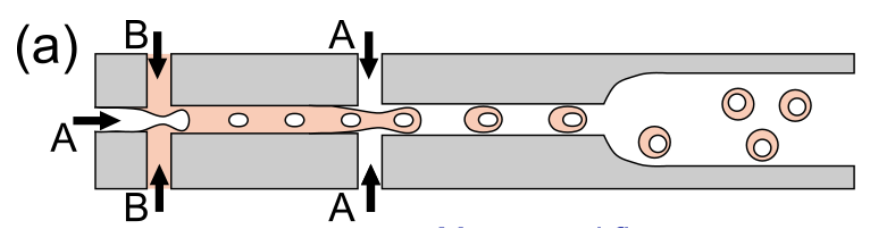

droplet production:

A: water/surfactant $(c<\mathrm{CMC})$

B: oil
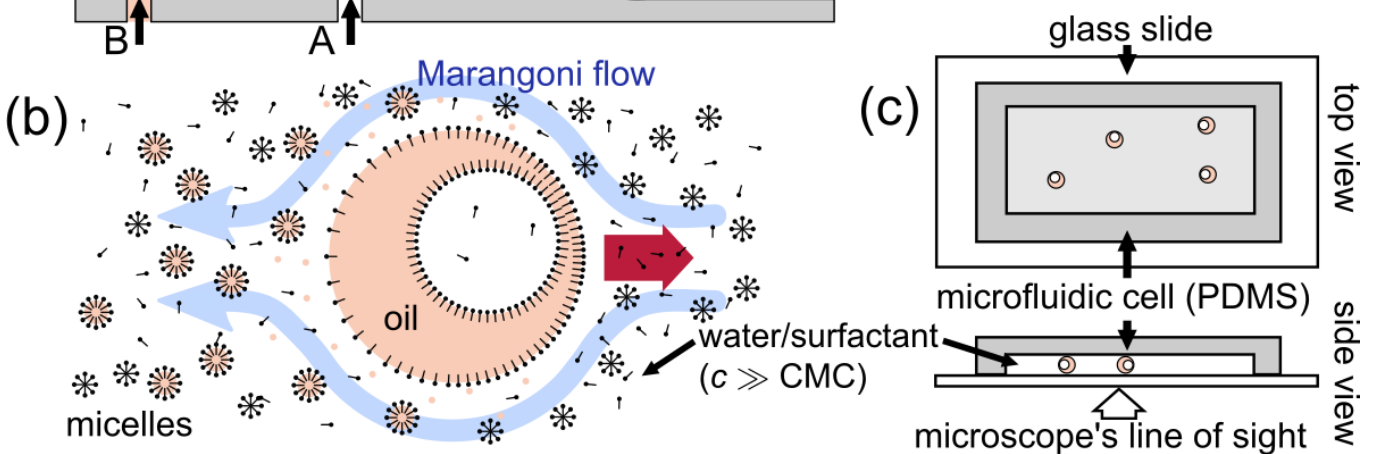

Figure 2.1 - Droplet production and setup schematics. (a) Double emulsion production via microfluidic flow junctions. (b) Droplet propulsion via a self-sustaining Marangoni gradient in the interface. (c) Microfluidic cell and inverted microscope setup for quasi-2D observation. All schematics are not to scale, spherical shells and micelles are represented by their $2 D$ cross-section.

drives the droplet motion [13, 58, 67] (Fig. $2.1 \mathrm{~b}$ ). Swimming droplets shed persistent trails of oil-filled micelles, from which they are subsequently repelled [89].

We use the nematogen $5 \mathrm{CB}$ as the oil phase, and solutions of the anionic surfactant TTAB as the aqueous phases, where the internal core droplet is submicellar $(c=$ $0.75 \mathrm{CMC}$ ), and the external swimming medium is supramicellar $(c>30 \mathrm{CMC})$. We mass-produce highly monodisperse oil droplet shells using consecutive microfluidic cross-junctions in flow-focusing configuration [106], (Fig. 2.1 a, also see section 2.4.1) and observe them in quasi-2D microfluidic cells under videomicroscopy (Fig. 2.1 c).

Despite the displacement of the aqueous core towards the shell boundary (Fig. $2.2 \mathrm{a}$ ), the shells self-propel stably and reproducibly for long times, dissolving down to thin shells with a minimum stable shell/core radii fraction of $R_{s} / R_{c} \approx 1.05$. The life stages of these self-propelling shells (Fig. $2.2 \mathrm{~b}$ and c) fall into three regimes: (I) 'Shark-fin' meandering. At early times, the core is small compared to the shell diameter (Fig. $2.2 \mathrm{~b}$, top) and is deflected considerably from the polar axis of the shell, resulting in a meandering instability. (II) Thin shells. As the shell thins, eventually there is little room for significant asymmetry in the shell-core arrangement (Fig. 2.2 b, bottom). During this stage, the motion grows noisy while the speed decreases, until propulsion stops. (III) Single Emulsion. On reaching a critical minimum thickness, the shell bursts, reconstituting into a single oil droplet. From a comparison of pre- and post-burst radii, we estimate that the average shell thickness at this point is less than $1 \mu \mathrm{m}$ 
(a)

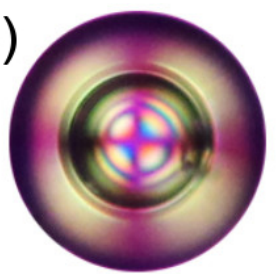

resting

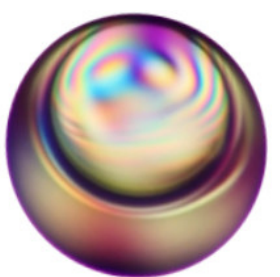

swimming

(c)

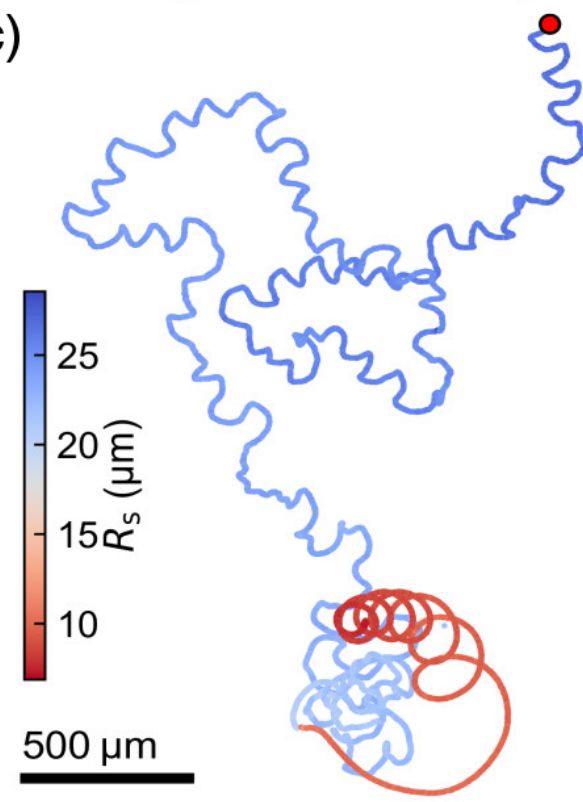

(b)

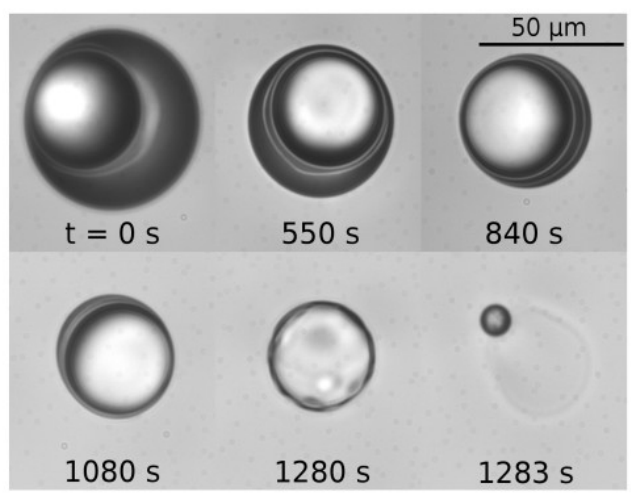

(d)

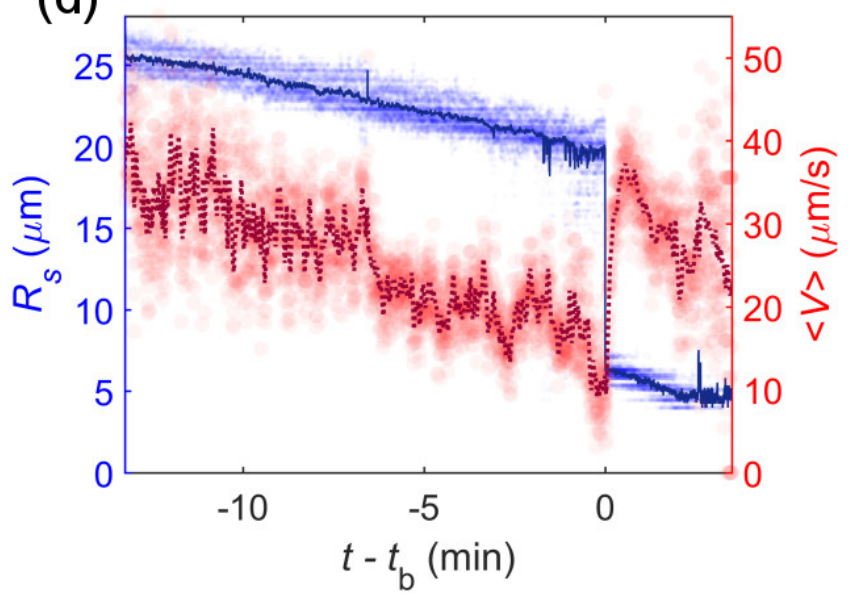

Figure 2.2 - Life stages of active shells. (a) Polarized images of resting and swimming shell. (b) Video stills of droplet life stages. (c) Example trajectory colored by shell radius $R_{s}$, recorded over $40 \mathrm{~min}$. The sudden change of color from blue to red corresponds to the burst moment. (d) Average speed $V$ (dotted, red) and radius $R_{s}$ (solid, blue) for 13 shells, time $t$ relative to bursting time $t_{b}$ (scatter plots: values for all experiments).

(Fig. $2.2 \mathrm{~d}$ ). The droplet then propels with an undisturbed internal convection, leading to a sudden increase in speed (Fig. $2.2 \mathrm{~d}$ ), and a curling motion as observed in nematic single emulsions [116](Fig. 2.2 c).

In contrast to these reproducible stages in nematic shells, we find that under otherwise identical conditions, shells made from isotropic oils (CB15 or 5CB/BPD, see section 2.4.1) burst significantly earlier. Fig. 2.3 a shows burst statistics for $5 \mathrm{CB}$ shells, where below the clearing point $\left(T<34.5^{\circ} \mathrm{C}\right.$, nematic) shells survive for long times, whereas above the clearing point $\left(T>34.5^{\circ} \mathrm{C}\right.$, isotropic), most droplets do not reach the thin shell stage.

We attribute the shell stability to a nemato-elastic energy barrier: 5CB molecules arrange to minimize the elastic energy associated with the deviations from a uniform 

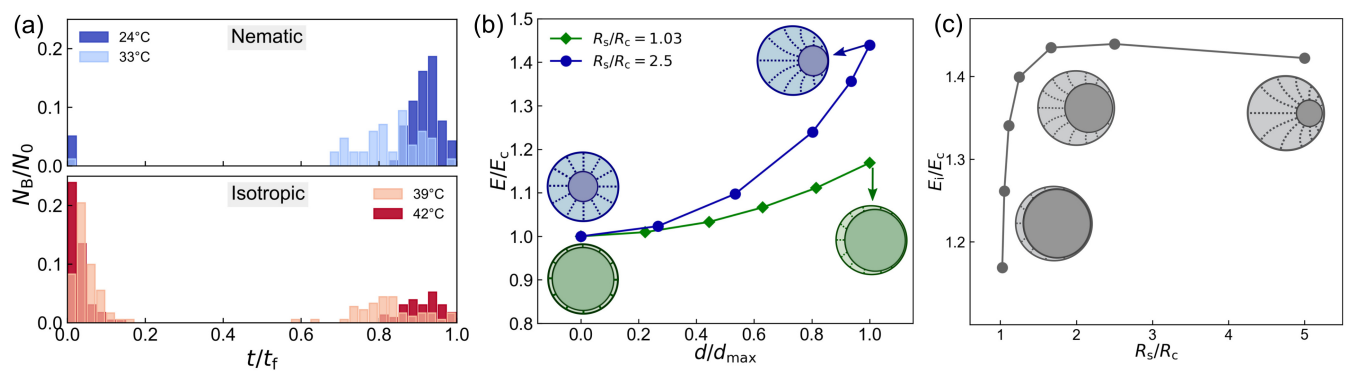

Figure 2.3 - Stabilization of the active shells. (a) Burst statistics (number of bursts $N_{B}$ normalized to initial number of shells $N_{0}$ ) for shells below and above the clearing point, plotted against time $t$ normalized to final time $t_{f}$ with no remaining shells $\left(t_{f}\left(24^{\circ} \mathrm{C}\right) \approx 45 \mathrm{~min} ., t_{f}\left(39^{\circ} \mathrm{C}\right) \approx 10 \mathrm{~min}\right.$. , initial $\left.R_{s}=30 \mu \mathrm{m}, R_{c}=18 \mu \mathrm{m}\right)$ (b) Elastic energy $E / E_{c}$ ( $E_{c}$ : core at center of droplet) as a function of core displacement $d / d_{\text {max }}$, with $d_{\max }=R_{s}-R_{c}-100 \mathrm{~nm}$, for two different ratios $R_{s} / R_{c}$. (c) Ratio of elastic energies $E_{i} / E_{c}$ ( $E_{i}$ : core droplet close to outer shell interface) against ratio of radii $R_{s} / R_{c}$, using $R_{c}=25 \mu \mathrm{m}$ and $R_{s}$ shrinking from $125 \mu \mathrm{m}$ to $25.6 \mu \mathrm{m}$. Schematics are illustrative, illustrated radius values do not directly correspond to the simulation parameters.

director field imposed by the boundary conditions (here homeotropic anchoring [117]). In a resting shell, this causes a radially symmetric arrangement of the director field [118] with the aqueous core at the center. In a moving shell the internal flow drives the core off-center: the director field is therefore distorted both by the displacement of the core and the flow field, such that the stored elastic energy is increased.

To estimate the competing forces we numerically simulated the director field inside the shell and calculated the elastic energy $E$ stored in a resting shell with a core displaced by a distance $d$. We applied a common numeric minimization technique [119, 120] based on the $\mathbf{Q}$ tensor representation [121] of the nematic director field (see section 2.4.3). The tensor elements of a uniaxial nematic with scalar order parameter $S$ and local director $\mathbf{n}$ are given by:

$$
Q_{j k}=\frac{S}{2}\left(3 \mathbf{n}_{j} \mathbf{n}_{k}-\delta_{j k}\right)
$$

Since topological defects are not present in the director field of our shells, we neglected a variation of the magnitude of $S$ and assumed a constant value $S=1$. For the calculation of the elastic energy density $f_{e}$ we used the one-constant approximation of the nematic elasticity, i.e., $K_{\text {splay }}=K_{\text {twist }}=K_{\text {bend }}=K$. Then, $f_{e}$ is obtained as:

$$
f_{e}=\frac{K}{9} Q_{j k, l} Q_{j k, l}
$$


where, $Q_{j k, l}=\partial_{l} Q_{j k}$. The total elastic energy $E$ is then calculated by integration over the shell volume $\Omega$ :

$$
E=\int_{\Omega} f_{e} \mathrm{~d} \Omega .
$$

As shown in (Fig. $2.3 \mathrm{~b}$ ), we find that $E$ increases by a factor of $E_{i} / E_{c} \approx 1.4$ when the core droplet is located at the outer interface $\left(E=E_{i}, d=d_{\max }\right)$, as compared to the centered configuration $\left(E=E_{c}\right)$. Remarkably, we find only a minor dependence on the thickness of the nematic shell. $E_{i} / E_{c}$ drops significantly towards unity only for $R_{s} / R_{c}<1.1$ (Fig. $2.3 \mathrm{c}$ ), i.e., the elastic energy barrier vanishes only in the limit of zero shell thickness.

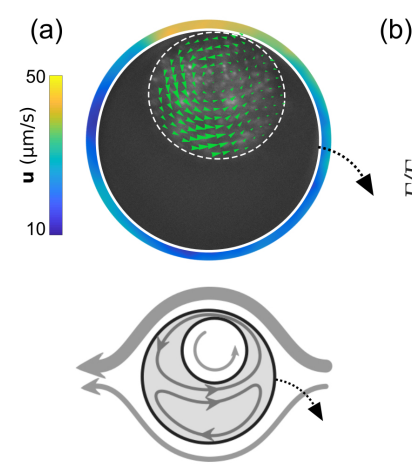

(d) No core; Radial hedgehog defect

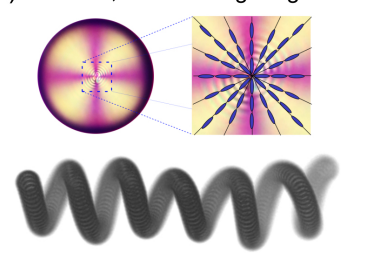

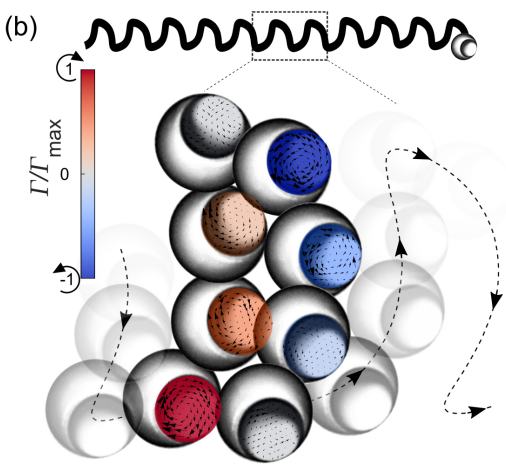

(e) One core; No defect

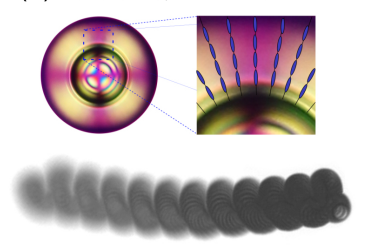

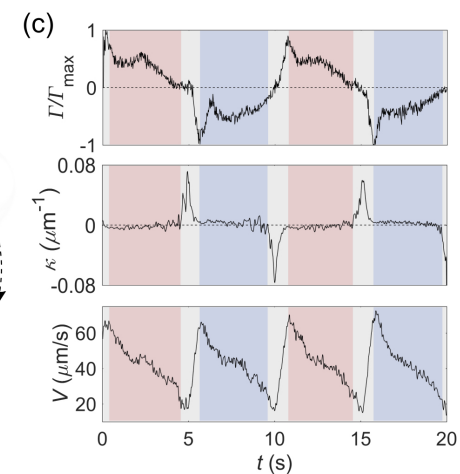

(f) Two cores; Hyperbolic hedgehog defect

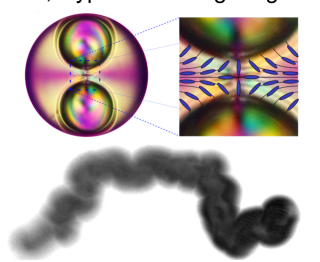

Figure 2.4 - Swimming behavior: (a) Top: PIV data for flow at the outer interface and in the core. Bottom: schematic of core and flow arrangement. (b) Top: 'shark-fin' meandering of swimming trajectory over 2 minutes $\left(R_{s}=36 \mu \mathrm{m}\right)$; the shell switches periodically between clockwise and anticlockwise turns. Bottom: zoomed in view of the shell-core alignment with the swimming trajectory, with superimposed core flow fields and color-coded circulation. (c) Core circulation $\Gamma / \Gamma_{\max }$, local curvature $\kappa$, and shell speed $V$ versus time over four meandering periods. (d), (e), and (f) show the nematic structure (top, polarized images of the droplets at rest with overlaid director field schematic) and $3 D$ swimming trajectories (bottom, multiple exposure micrograph captured over $60 \mathrm{~s}$ ) for a no core droplet (d), a single core droplet (e) and a two core droplet (f). See supporting movie $S 8$.

We calculate the elastic force $F_{e}=\partial E / \partial d$ acting on a core displaced to the boundary of a $5 \mathrm{CB}$ shell $[122,123]$ to be of the order of $\approx 100 \mathrm{pN}$. This is equivalent to the 
Stokes drag [13],

$$
F_{S}=2 \pi R_{c} v \frac{2 \eta_{5 \mathrm{CB}}+3 \eta_{a q}}{1+\eta_{a q} / \eta_{5 \mathrm{CB}}}
$$

acting on an aqueous core moving through bulk $5 \mathrm{CB}$ at $v \approx 6 \mu \mathrm{m} / \mathrm{s}$, which is comparable to the velocity of the convective flow in our shells. We propose that the nematoelastic repulsion provides a significant, although not insurmountable, barrier against coalescence.

We have analyzed the meandering dynamics by simultaneously tracking the circulation of the flow inside the core $\Gamma(t)$, local trajectory curvature $\kappa(t)$, and propulsion speed $V(t)$ (Fig. $2.4 \mathrm{a}-\mathrm{c}$ ). In quasi-2D confinement, the core is trapped off-axis inside the convective torus, where it co-rotates with the convective flow, as shown by the core flow and color coded $\Gamma$ values in Fig. $2.4 \mathrm{a}$, b. In this arrangement, there is less viscous resistance to the driving interfacial flows in the part of the shell containing the core, resulting in asymmetric flow with respect to the direction of motion (shown by the color bar in Fig. 2.4 a), and a curved trajectory. This eventually curves the shell back towards its own trail, where chemotactic repulsion causes $V$ and $\Gamma$ to slowly decay and then abruptly reverse - the tip of the 'shark-fin' motion (Fig. 2.4 b, c). This abrupt reorientation corresponds to a spike in the local curvature and is followed by a sharp acceleration (Fig. 2.4c), caused by repulsion from the local gradient of filled micelles. Due to the flow reversal, in the co-moving reference frame the core has now switched sides and the shell curves in the opposite direction, once again towards its own trail. We distinguish three time scales: a short timescale $(\approx 1 \mathrm{~s})$ for autochemotactically driven abrupt reorientation, an intermediate timescale $(\approx 5 \mathrm{~s})$ for the curved motion between two shark-fin tips, and a long timescale (>100s) corresponding to the persistent motion imposed by the chemical field in the trail of the shell (cf. Appendix, Fig. 2.7).

To further investigate the role of the core in breaking the flow symmetry, we have additionally experimented in 3D bulk media, using deep microfluidic wells and matching the density of oil and swimming media by substituting a fraction of water with deuterated water in the surfactant solution. We compared the dynamics of droplets with zero, one and two cores (Fig. $2.4 \mathrm{~d}-\mathrm{f}$ ). With no core, we reproduce previous findings [116], where the displacement of the radial 'hedgehog' defect induces a torque on the droplet. Given the freedom of a third dimension, the droplet is not arrested by its own trail and does not reverse its direction, resulting in helical trajectories. With one core, we observe similar behavior, with the core precessing around the axis of motion. Shells propel in more tightly wound helices than single emulsions, which can 
be understood in terms of the torque applied by the respective viscous anisotropy: for shells, it is the viscosity ratio of oil and water, $\eta_{2}(5 \mathrm{CB}) / \eta\left(\mathrm{H}_{2} \mathrm{O}\right) \approx 50$; in contrast, for single emulsions [116], it refers to the intrinsic viscous anisotropy of a nematic liquid crystal $\eta_{2}(5 \mathrm{CB}) / \eta_{\text {iso }}(5 \mathrm{CB}) \approx 3$ [123]. With two cores, this broken symmetry argument does not hold, and thus we are able to rectify the meandering motion.

While a single-core nematic shell is defect free and spherically symmetric at rest, double core shells have a fixed axis set by the two cores, with a topological charge of +1 resolved by a hyperbolic hedgehog defect, or a defect loop [124-126]. This defect provides a barrier against core coalescence [127]. Hence, as in the single-core case, the shell thickness shrinks to $\approx 1 \mu \mathrm{m}$ until the shell bursts (Fig. 2.5 a). The most likely flow field configuration inside a moving double-core shell is with both cores trapped on opposite sides of the convection torus and no symmetry breaking mechanism or curling. Instead, the shell moves perpendicularly to the core alignment, with some rotational fluctuations (demonstrated in quasi-2D, Fig. 2.5 b).

Changing the topology of the liquid crystal, e.g. by controlling the number of cores, provides one method to rectify the propulsion dynamics. However, based on our work on single emulsions $[89,103]$, we have further options to guide self-propelling shells and improve their utility as cargo carrying vessels and sensors, by exploiting microfluidic topography $[128,129]$ and chemical gradients. First, topographical guidance: Fig. $2.5 \mathrm{c}$ shows a shell swimming along a wall, turning both convex corners without detachment and concave corners without arrest (further examples in Fig. 2.9). Second, chemotactic guidance: In Fig. 2.5 d, crystalline surfactant ('attractant') is allowed to dissolve into a quasi- $2 \mathrm{D}$ cell. The resulting gradient extends $\approx 1 \mathrm{~mm}$ into the cell, attracting the shells, doubling their speed and rectifying the meandering instability.

\subsection{Conclusion}

In conclusion, we have developed a versatile platform for microscopic cargo delivery: self-propelling droplet shells. While motility induces convection that acts to destabilize these cargo vessels, we have demonstrated through experiments and simulations that nemato-elasticity can be employed as a topologically stabilizing agent, a fact we anticipate will be utilized in novel designs of microreactors and artificial cells. We have also provided pathways for guiding the trajectories of these droplets, through both chemical signaling and topography. Finally, we have analyzed the interesting swimming behavior of these self-propelling shells, and anticipate that the understanding of the 
(a)
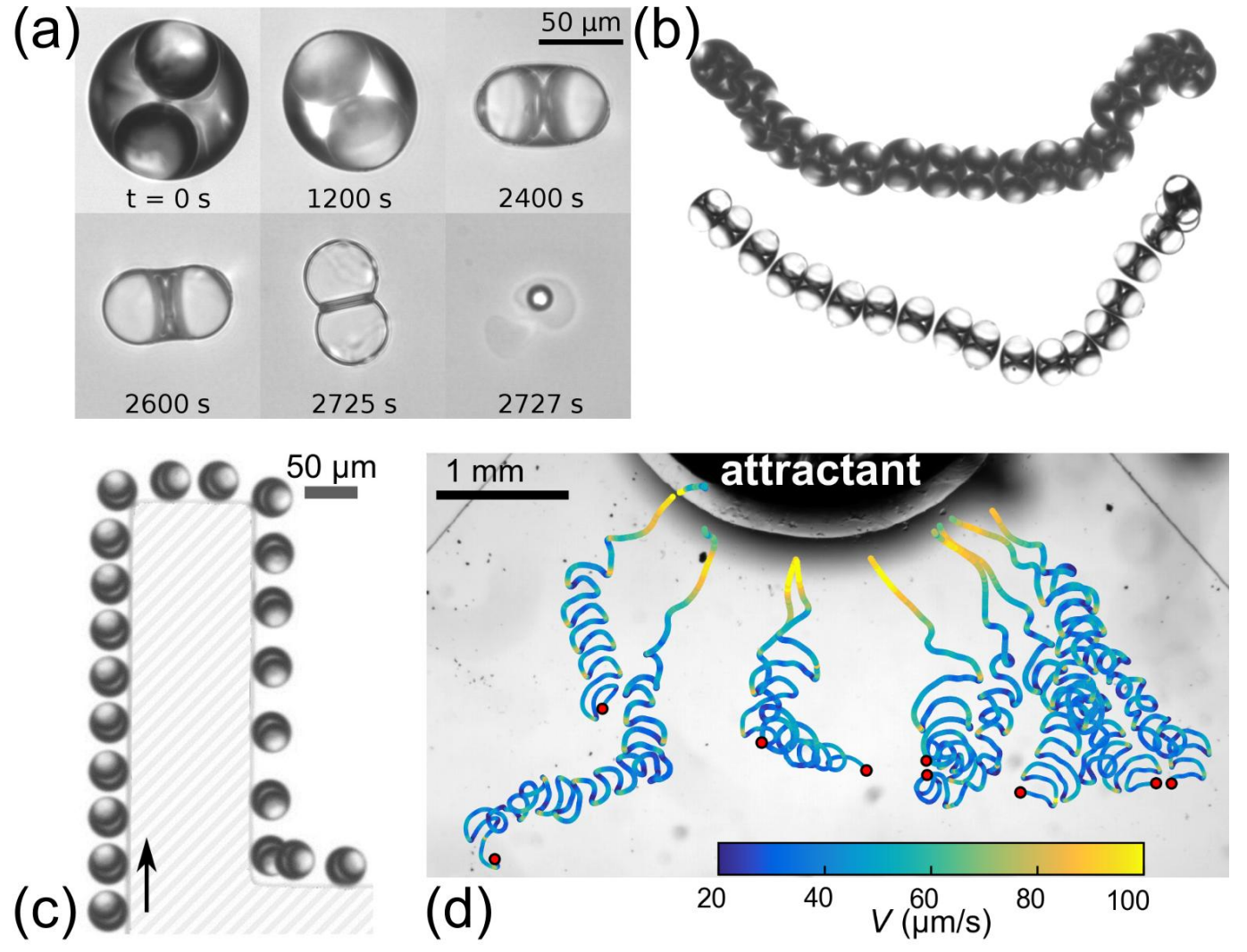

Figure 2.5 - Control of shell dynamics. Micrographs of shells with two cores, showing (a) long-time stability and (b) no meandering in $2 D$ confinement (multiple exposure micrographs at two shell thicknesses). Due to the larger initial volume of the oil phase, survival times are increased compared to Fig. $2.2 \mathrm{~b}$. (c) Topographical guidance by walls. Multiple exposure micrograph taken over 65 seconds at 3 second intervals. (d) Chemotaxis: diffusing surfactant guides shells to the left (trajectories colored by shell speed $V$ ).

rich 'shark-fin' meandering dynamics will impact the design of artificial microswimmers, where swimming behavior can be tweaked by tuning the routes for spontaneous symmetry breaking.

Financial support from the Deutsche Forschungsgemeinschaft (SPP1726 "Microswimmers") is gratefully acknowledged. We thank Stephan Herminghaus for stimulating discussions and Julien Petit for invaluable experimental advice. 


\subsection{Appendix}

\subsubsection{Double emulsion fabrication}

Our emulsions comprise the nematic liquid crystal 4-pentyl-4'-cyano-biphenyl (5CB) and an aqueous solution of the surfactant tetradecyltrimethylammonium bromide (TTAB). Where required, we match the densities between the oil $\left(\rho_{5 C B}=1.022 \mathrm{~g} \mathrm{ml}^{-1}\right.$ at $24^{\circ} \mathrm{C}$ ) and surfactant solution phases by $\mathrm{D}_{2} \mathrm{O}\left(\rho_{D_{2} \mathrm{O}}=1.107 \mathrm{~g} \mathrm{ml}^{-1}\right)$ admixture, with adjustments for TTAB content. In separate experiments, we created isotropic double emulsions, using: the branched 5CB isomer CB15 ((S)-4-Cyano-4'-(2-methylbutyl)biphenyl, Synthon Chemicals); or a 10:1 volumetric ratio of 5CB and BPD (Bromopentadecane, Sigma-Aldrich). These shells were highly unstable when active, and were not examined further.

We generate and observe double emulsion droplets in microfluidic PDMS chips on glass slides; fabricated in-house using standard soft lithography methods [130]. We follow the recipe of Petit et al. [106], for creating monodisperse water-in-oil-in-water emulsions, where the chips consist of two sequential cross-shaped flow junctions, for water-in-oil followed by oil-in-water droplet pinch-off (Fig. 2.6). The number of cores, as well as the core and shell diameters, are set by the flow rates [131]. Shell and core radii were produced in the range of $R_{s}=20-130 \mu \mathrm{m}$ and $R_{c}=10-50 \mu \mathrm{m}$ respectively. Typical flow rates were in the ranges 10-30, 60-150, and 200-500 $\mu \mathrm{hr}^{-1}$ for the core aqueous phase, oil phase, and external aqueous phase respectively.

To ensure the oil phase did not wet the initially hydrophobic PDMS walls, the outer flow channels were hydrophilised prior to droplet production. This was achieved by drawing a sequence of liquids through the outermost channel via a vacuum pump induced pressure gradient. The sequence was as follows: a 1:1 volumetric ratio of hydrochloric acid ( $\mathrm{HCl}$ at 37 wt.\%, Sigma-Aldrich) and hydrogen peroxide $\left(\mathrm{H}_{2} \mathrm{O}_{2}\right.$ at 30 wt.\%, Sigma-Aldrich) for 2 minutes; Milli-Q water for 30 seconds; 5 wt.\% aqueous solution of poly(diallyldimethylammonium chloride) (PDADMAC, average molecular weight $M_{w} \approx 100,000-200,000 \mathrm{~g} \mathrm{~mol}^{-1}$, Sigma-Aldrich), for 2 minutes; 2 wt.\% aqueous solution of poly(sodium 4-styrenesulphonate) (PSS, $M_{w} \approx 1,000,000 \mathrm{~g} \mathrm{~mol}^{-1}$, SigmaAldrich) for 2 minutes.

During production and in storage, both the internal and external aqueous phases contain a submicellar concentration of TTAB (0.1 wt.\%): sufficient to stabilize the interface, but insufficient to induce solubilisation $(\mathrm{CMC}=0.13$ wt.\%). 5CB double emulsion droplets at room temperature $\left(T=24^{\circ} \mathrm{C}\right)$ are stable against coalescence for 


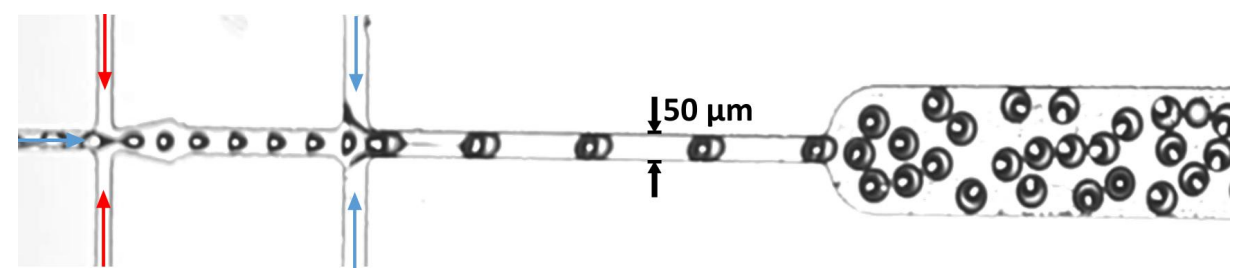

Figure 2.6 - Screenshot of a microfluidic chip producing double emulsion droplets $\left(R_{s} \approx 30 \mu \mathrm{m}\right)$. Blue and red arrows indicate flow of aqueous and oil phases respectively. The channel height is $50 \mu \mathrm{m}$.

several months.

\subsubsection{Observation and analysis}

We performed experiments in two geometries, quasi-2D PDMS reservoirs with typical dimensions of $7.5 \mathrm{~mm} \times 5 \mathrm{~mm} \times 50 \mu \mathrm{m}$, and deeper wells of size $10 \mathrm{~mm} \times 6 \mathrm{~mm} \times 100$ $\mu \mathrm{m}$. We used bright field microscopy to record shell trajectories, polarized microscopy for nematic order and higher resolution fluorescence microscopy to measure flow fields using $0.5 \mu \mathrm{m}$ fluorescent tracer colloids. For polarised microscopy we used a Nikon Eclipse LV100 microscope equipped with a digital camera (EOS 600d, Canon). Crossed polarisers with an added $\lambda$ retardation plate were used to visualise the nematic director field inside the liquid crystal phase. We used two inverted microscopes (Olympus IX-73 and IX-81) with either a Grasshopper (GS3-U3-41C6M-C) greyscale camera $(2048 \times 2048$ pixels $)$ or a Canon (EOS 600d) digital camera $(1920 \times 1080$ pixels $)$ for regular visible light microscopy recording images at 4-24 fps and 4-40× magnification for tracking the swimmers. The fluorescent imaging was done on an IX-73 inverted microscope at 24 fps and $20 \times$ magnification for PIV measurements around the shells and at 48 fps and $40 \times$ magnification for PIV inside the core. We extracted shell positions from video microscopy data using standard Python libraries for numpy, PIL and opencv (Scripts available on request). Essential steps are background correction, binarisation, blob detection by contour analysis and minimum enclosing circle fits. We calculated trajectories and speeds using a simple next neighbour algorithm [132]. We identified bursting times by frame-by-frame inspection. We estimated core position and orientation angles from high resolution video data by semi-automatically determining circular shell and core outlines and comparing the line of centers to the trajectory normal. The mean squared displacement of the shell trajectory was calculated as

$$
\left\langle(\Delta r)^{2}\right\rangle_{t}=\left\langle\left[\mathbf{r}\left(t_{0}+t\right)-\mathbf{r}\left(t_{0}\right)\right]^{2}\right\rangle_{t_{0}},
$$


where $\mathbf{r}$ is the position vector of the shell. The angular or velocity autocorrelation function was calculated as

$$
C(t)=\left\langle\frac{\mathbf{V}\left(t_{0}+t\right) \cdot \mathbf{V}\left(t_{0}\right)}{\left|\mathbf{V}\left(t_{0}+t\right)\right|\left|\mathbf{V}\left(t_{0}\right)\right|}\right\rangle_{t_{0}},
$$

and the (signed) local curvature of the trajectory was defined as

$$
\kappa=\frac{x^{\prime} y^{\prime \prime}-y^{\prime} x^{\prime \prime}}{\left(x^{\prime 2}+y^{\prime 2}\right)^{3 / 2}},
$$

where the prime denotes derivation with respect to time.

For illustration, we have included speed, orientation, mean squared displacement $\left\langle(\Delta r)^{2}\right\rangle_{t}$ and velocity autocorrelation $C(t)$ data in Fig. 2.7 for one trajectory (see also Fig. 3 in the main text). The periodic meandering reflects in a dip in the $\left\langle(\Delta r)^{2}\right\rangle_{t}$, which is $\propto t^{2}$ for long times, approaching ballistic motion. The absence of rotational noise reflects in a periodic, non-decaying character of $C(t)$.
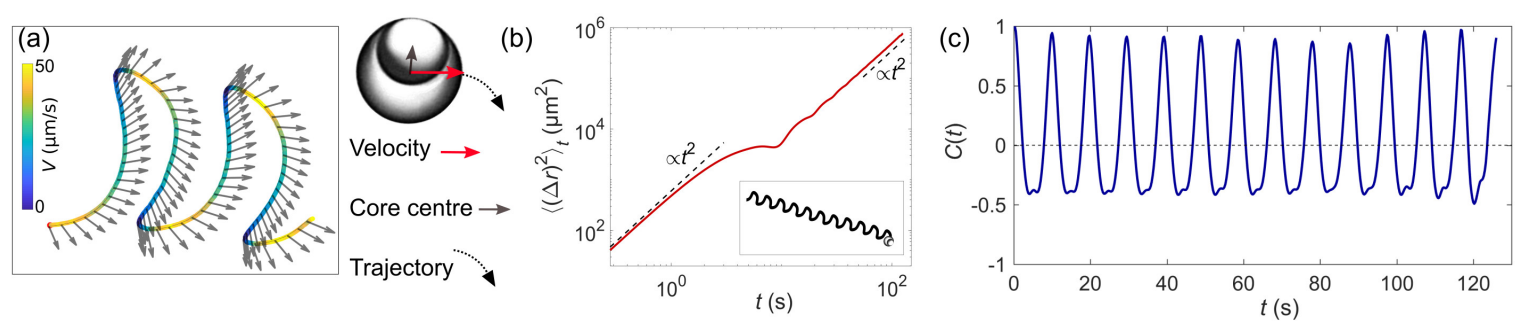

Figure 2.7 - Characterisation of the swimming behaviour. (a) The magnitude of the swimming velocity. The vectors show the location of the core at each time. The sudden acceleration right after the abrupt reorientation corresponds to the autochemotactic repulsion from the trail of filled micelles. (b) Mean squared displacement of an individual swimmer with the trajectory as the inset. The dip corresponds to the meandering period.

(c) Velocity auto-correlation function of the same swimmer.

For the PIV analyses in Fig. $3 \mathrm{a}$ and Fig. $4 \mathrm{~b}$. the shell was pinned in a shallow cell and the aqueous phase was seeded with $0.5 \mu \mathrm{m}$ fluorescent tracer colloids (Thermo Fisher Scientific). For PIV inside the core, the core was seeded with the same tracers, the core position was tracked manually and the time-resolved PIV images were collected. All PIV analyses and flow calculations were performed via the MATLAB based PIVlab interface [133]. Additional custom MATLAB scripts were written to calculate the circulation of the flow inside the internal aqueous droplet [134]:

$$
\Gamma_{i}(t)=\hat{\mathbf{z}} \cdot\left[\sum_{(x, y)_{i}} \boldsymbol{r}_{i}(x, y) \times \mathbf{u}(x, y, t)\right],
$$


where $\boldsymbol{r}_{i}$ is the vector from the core center to $(x, y)$ inside the projected area of the core on the $\mathrm{x}-\mathrm{y}$ plane and $\mathbf{u}$ is the velocity vector field inside the core.

\subsubsection{Numerical simulation}

The estimated elastic energies $(E)$ plotted in Fig. 2 b, c were calculated by applying a common numeric minimisation technique $[119,120]$ based on the $\mathbf{Q}$ tensor representation [121] of the nematic director field, where we have assumed that the 3 nematic elasticity constants are equal, and the scalar order parameter is equal to 1 in a defect-free shell. More details of these calculations have been included in the main manuscript.

In order to determine the structure possessing the minimal value of $E$, the shell was mapped onto a cubic grid with $256^{3}$ nodes. The tensor components at each node were assumed to relax to their equilibrium values according to a simplified equation of motion, governed only by the rotational viscosity $\gamma_{1}$. In a discretised form with time steps $\Delta t$ we have:

$$
\gamma_{1} \frac{\Delta Q_{j k}}{\Delta t}=-\frac{\delta f_{e}}{\delta Q_{j k}} .
$$

The right-hand side of the above equation represents the functional derivative of $f_{e}$ with respect to tensor component $Q_{j k}$. Details of the numerical procedures and explicit expressions for the various functional derivatives are given in [119]. For the boundary conditions we assumed strict homeotropic anchoring of the director at all interfaces, i.e., for the nodes located at the outer or inner surface of the shell, the orientation of the nematic director at the interface is set perpendicular to the interface and held constant (so-called strong anchoring conditions). We varied the shell size and the position of the inner aqueous droplet over a wide range and determined the corresponding equilibrium structures and values of $E$. Fig. 2.8 illustrates some examples. We note that we considered here solely the interplay between the shell size, the position of the internal core, and the elastic energy of the nematic director field. We neglected the internal convectional flow which is present in our self-propelling shells, which certainly leads to additional effects on the structure of the director field. 
(a)

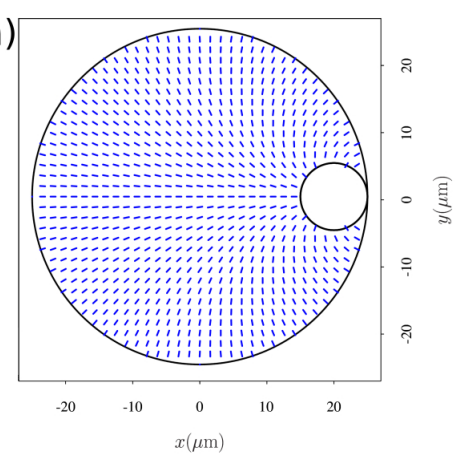

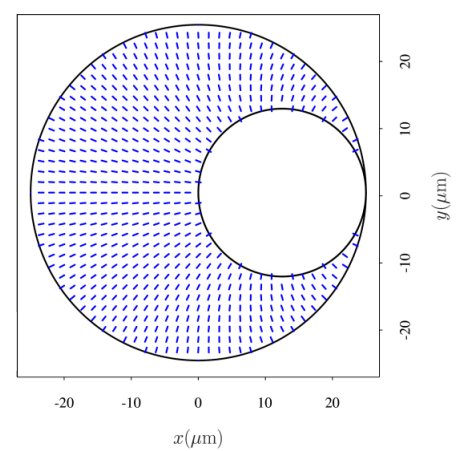

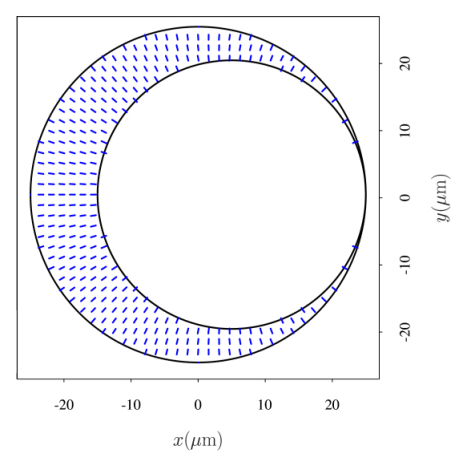

(b)
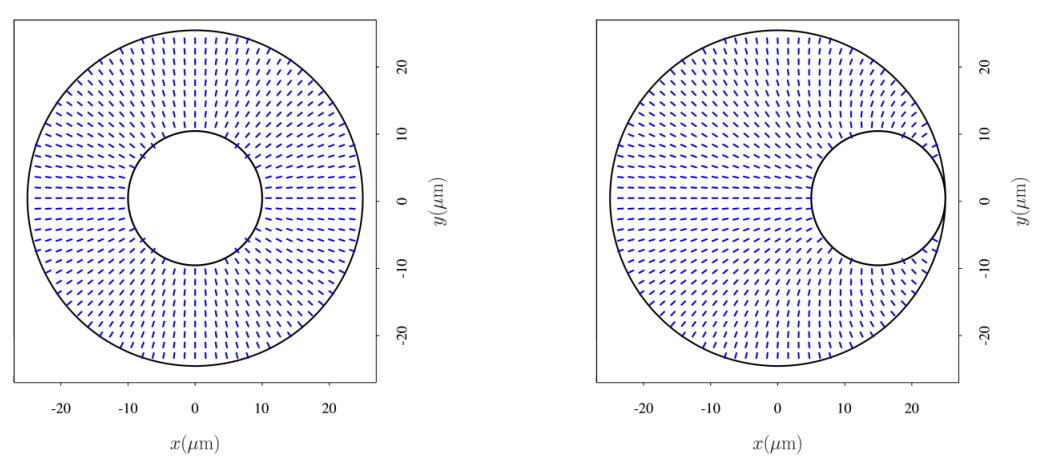

Figure 2.8 - Simulation of the director field. Row (a) shows the effect of size ratio for the maximum displacement of the core from the center, row (b) shows the uniform (left) and distorted director fields (right), indicating the effect of core displacement on the deviation of the director field.
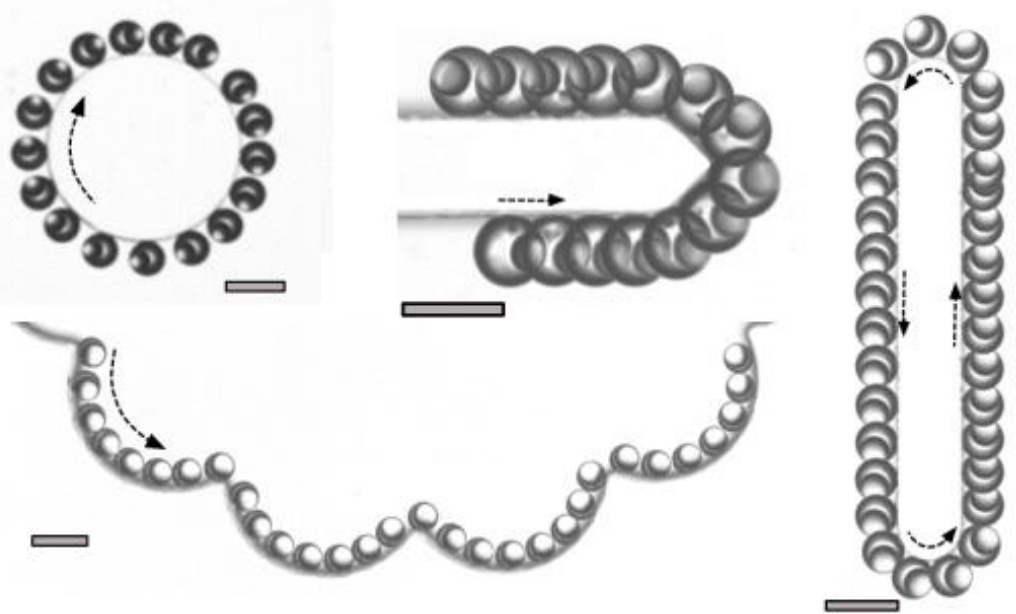

Figure 2.9 - Further examples of topographical guidance of the shells, including around edges and corners, cf. Fig. 4, main text. The scale bar is $100 \mu \mathrm{m}$. 
Supplementary movies are available for viewing under http://asm.ds.mpg.de/index.php/media/.

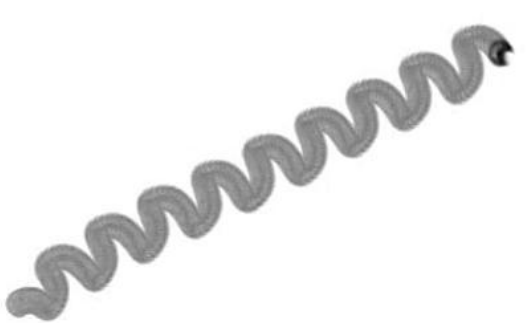

Figure 2.10 - Movie S5 showing one shell $\left(R_{s} \approx 30 \mu \mathrm{m}, 131 \mathrm{~s}\right.$, speed $\times 6$ at $24 \mathrm{fps}$ ) 'shark-fin' meandering, with high persistence and periodicity, as well as a 'curling' single emulsion droplet, in a uniform bulk environment.

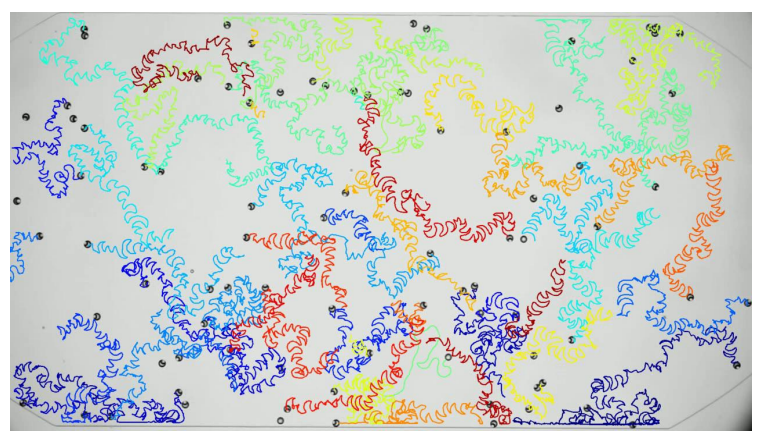

Figure 2.11 - Movie S6 showing selected trajectories for an ensemble of 'shark-fin' meandering shells $(500 \mathrm{~s}$, speed $\times 25$ at $10 \mathrm{fps}$, field of view $5.4 \times 3 \mathrm{~mm})$. Reorientation on long timescales is caused by the filled micelles in the wake of previously passing shells.

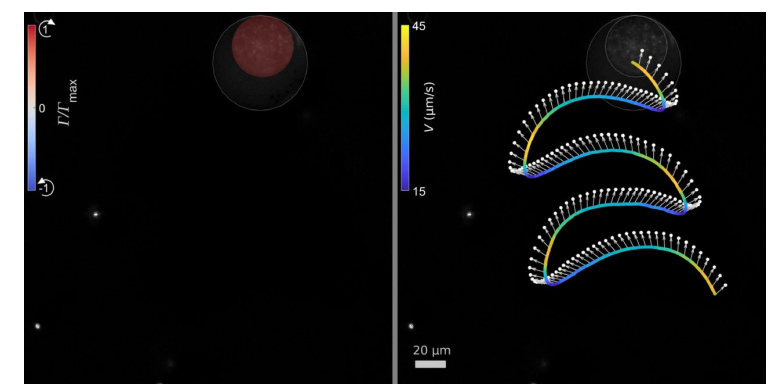

Figure 2.12 - Movie $S 7$ (255 s, speed x 1 at $24 \mathrm{fps}$ ) tracking circulation, speed and core orientation for a meandering shell, data corresponding to Fig. $3 \mathrm{~b}, \mathrm{c}$ in the main text.

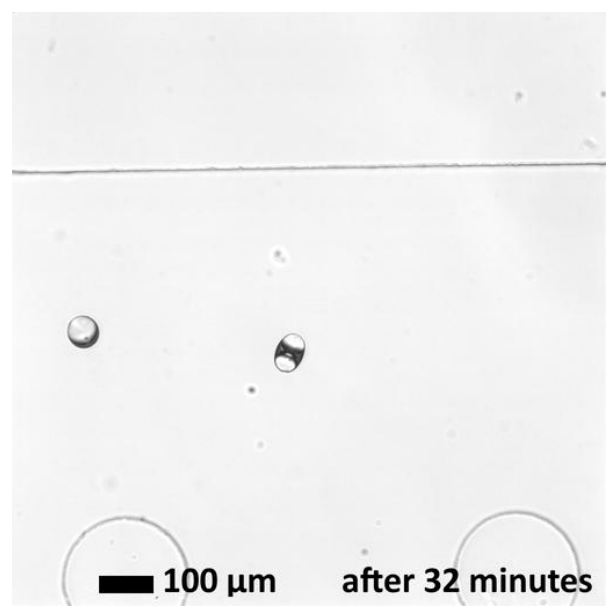

Figure 2.13 - Movie S8 (speed x 12 at $24 \mathrm{fps}$ ) showing the dynamics and life stages of a double core shell (see Fig. 4 in the main text). 



\title{
Chapter 3
}

\section{Emergence of bimodal motility in active droplets ${ }^{1}$}

\begin{abstract}
Artificial model swimmers offer a platform to explore the physical principles enabling biological complexity, for example, multi-gait motility: a strategy employed by many bio-microswimmers to explore and react to changes in their environment. Here, we report bimodal motility in autophoretic droplet swimmers, driven by characteristic interfacial flow patterns for each propulsive mode. We demonstrate a dynamical transition from quasi-ballistic to bimodal chaotic propulsion by controlling the viscosity of the environment. To elucidate the physical mechanism of this transition, we simultaneously visualize hydrodynamic and chemical fields and interpret these observations by quantitative comparison to established advection-diffusion models. We show that, with increasing viscosity, higher hydrodynamic modes become excitable and the droplet recurrently switches between two dominant modes due to interactions with the self-generated chemical gradients. This type of self-interaction promotes self-avoiding walks mimicking examples of efficient spatial exploration strategies observed in nature.
\end{abstract}

\footnotetext{
${ }^{1}$ This chapter is published in Hokmabad, B. V., Dey, R., Jalaal, M., Mohanty, D., Almukambetova, M., Baldwin, K. A., Lohse, D. and Maass, C. C. (2020). Stop-and-go droplet swimmers. arXiv preprint arXiv:2005.12721 and currently under review. B. V. H. designed the research, performed experiments and data analyses and wrote the manuscript. R. D. performed the theoretical analyses and wrote the manuscript. M. J. designed the research, performed experiments and wrote the Manuscript. D. M. and M. A. and K. A. B. performed experiments. D. L. and C. C. M. supervised the project and wrote the manuscript.
} 


\subsection{Introduction}

In response to physical constraints in nature, microorganisms have adapted and developed various locomotion strategies. Depending on cues from the environment, these strategies range from the more commonplace helical swimming $[135,136]$, run-andtumble, and switch-and-flick motility [137], to more sophisticated transient behaviours, e.g. peritrichous bacteria switching poles in response to a steric stress [138], octoflagellate microalgae exhibiting run-stop-shock motility with enhanced mechanosensitivity [139], and starfish larvae maximising fluid mixing, and thereby nutrition uptake, through rapid changes of ciliary beating patterns [140]. Such intricate gait-switching dynamics [141, 142] enable organisms to navigate in external flows [26, 143], to follow gradients [144] or to efficiently explore their environment $[145,146]$. Recent efforts in the development of synthetic swimmers have led to synthesis of systems that are capable of mimicking some of the aforementioned features of their natural counterparts such as rheotaxis [147, 148], chemotaxis [89, 149], and gravitaxis [150]. However, dynamic multi-modal motility in the absence of external actuation has not been explored before in artificial swimmers, and the mechanisms underlying unsteady behaviour in self-actuating systems are not well understood, particularly with respect to distinguishing states with increased random fluctuation from ones featuring true multimodal behavior[151, 152].

Paradigms for biomimetic artificial swimmers include autophoretic microswimmers, powered by chemical activity at their interface, which are able to generate long-living chemical gradients in the environment [149]. In this regard, droplet microswimmers driven by micellar solubilization [67], provide a sophisticated experimental realisation. Unlike most synthetic swimmers which are inherently asymmetric, active droplets are isotropic. Interfacial activity spontaneously breaks the symmetry, allowing for emergence of different flow patterns depending on the environmental parameters. Here we use such active droplets as model systems to demonstrate the physical principles guiding the emergence of multi-modal motility in response to changes in environmental conditions.

We show that active droplets adapt to an increase in the viscosity of the swimming medium by exhibiting increasingly chaotic motion- a counter-intuitive response given that increasing viscous stress generally tends to stabilise non-inertial dynamics. Using time-resolved in situ visualisation of the chemical and the hydrodynamic fields around the droplet interface, we found that the emergence of the chaotic dynamics correlates with the onset of higher hydrodynamic modes at increasing Péclet number $P e$. Once 
these higher modes prevail, the droplet exhibits an unsteady bimodal exploration of space triggered by its interaction with a self-generated, slowly-decaying chemical gradient. The conditions for the onset of this dynamical transition are quantitatively predicted by an advection-diffusion model for the transport of the chemical species, which takes into account the nonlinear coupling between the hydrodynamic and chemical fields. The visualisation technique and the findings presented here lay the groundwork for future investigations of emergent dynamics in active phoretic matter.

\subsection{Droplets propelled by micellar solubilisation}

Oil droplets that are slowly dissolving in supramicellar aqueous surfactant solutions can spontaneously develop self-sustaining gradients in interfacial surfactant coverage, resulting in Marangoni stresses which lead to self-propulsion [13]. This interfacial instability may be understood as follows (Fig. 3.1 a,b): During the solubilisation of the droplet, oil molecules migrate into surfactant micelles in a boundary layer around the droplet interface, causing the micelles to swell and take up additional surfactant molecules from the aqueous phase. This depletes the interfacial surfactant concentration, unless there are empty micelles present to replenish it by disintegration. The interfacial tension therefore increases with the local ratio of filled to empty micelles. Following an advective perturbation in the vicinity of the droplet, the radial symmetry of the filled micelle distribution is spontaneously broken; the resulting fore-aft asymmetry generates a surface tension gradient which drives the droplet towards more empty micelles leading to sustained self-propulsion, while leaving behind a trail of swollen micelles. Such spontaneous self-propulsion stemming from the advection-diffusion driven interfacial instability arises only if the Péclet number, $P e$, which characterises the ratio of advective to diffusive transport, exceeds a critical threshold [54, 57, 58, 73]. For the active droplet system, $P e$ can be shown to be a monotonically increasing function of the swimming medium (outer) viscosity $\mu^{o}$, here non-dimensionalised as $\mu=\mu^{o} / \mu^{i}$ using the constant inner viscosity $\mu^{i}$ :

$$
P e=\frac{V_{t} R_{d}}{D} \approx \frac{18 \pi^{2}}{k_{B} T} q_{s} r_{s}^{2} \zeta R_{d}^{2} \mu^{i}\left[\mu\left(\frac{2 \mu+3 \zeta / R_{d}}{2 \mu+3}\right)\right]
$$

where $V_{t}$ is the theoretical terminal droplet velocity in an external surfactant gradient $[43,54], R_{d}=30 \mu \mathrm{m}$ the droplet radius, $D=\frac{k_{B} T}{6 \pi r_{s} \mu^{\circ}}$ the diffusion coefficient for the surfactant monomer (length scale $r_{s} \sim 10^{-10} \mathrm{~m}$ ), $q_{s}$ the isotropic interfacial surfactant 
consumption rate per area, and $\zeta \sim 10 \mathrm{~nm}$ the characteristic length scale over which the surfactants interact with the droplet $[43,58]$ (see Appendix 3.9 for the derivation of eqn. (3.1)). In experiments, we controlled $\mu^{o}$ by using water/glycerol mixtures as the swimming medium (viscosity values in Appendix 3.9, Fig. 3.7). Increasing $\mu^{o}$ corresponds to an increase in $P e$, besides the increase in viscous stresses. Henceforth, we represent an increase in $\mu^{o}$ by this corresponding increase in $P e$, as tabulated by the colour map in Fig. 3.2. We note that in view of the necessary simplifications in the derivation of eqn. (3.1), all experimental $P e$ values should be regarded as approximate.
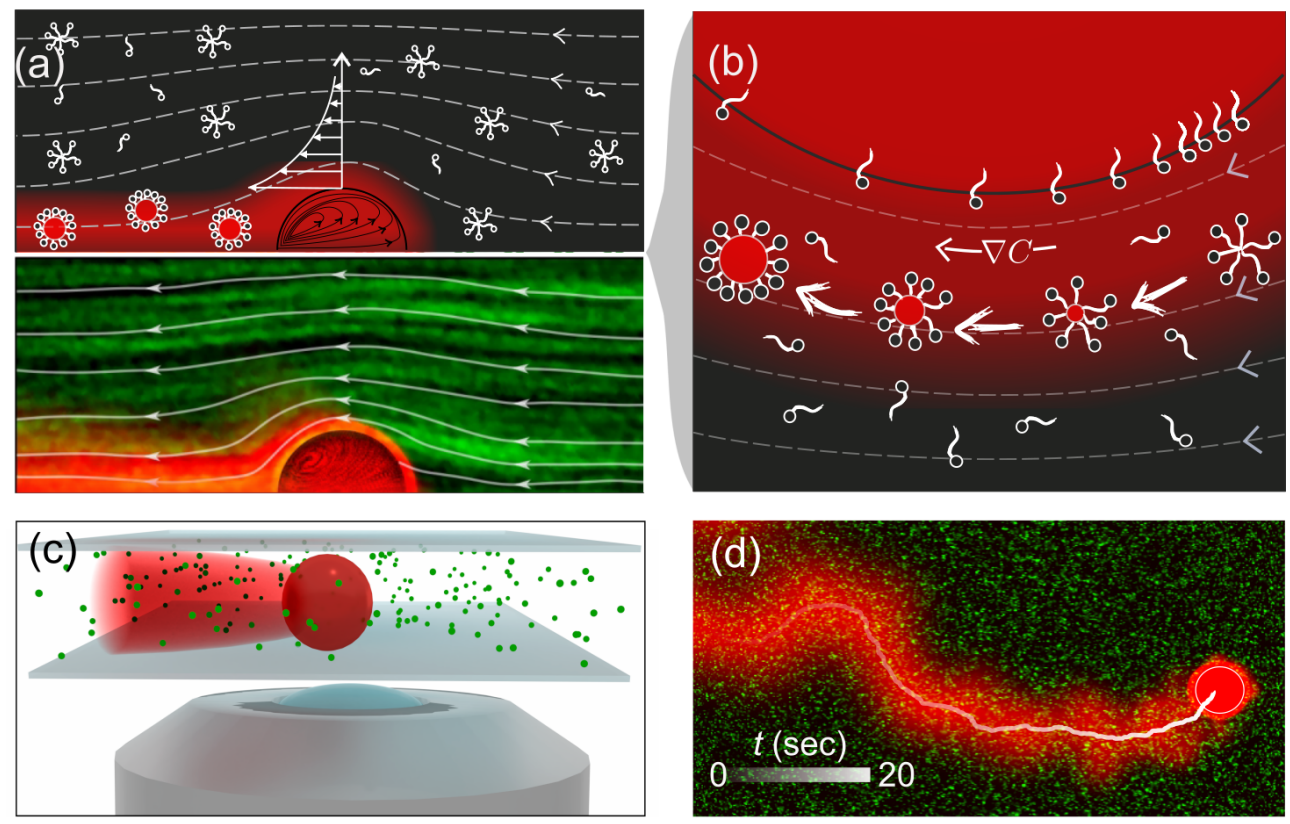

Figure 3.1 - Droplet propulsion mechanism and visualisation technique. (a) Top: Schematic illustration of the micellar solubilization of oil at the droplet interface leading to self-propulsion. Bottom: Streaks of tracers following the flow inside and outside of the droplet during 2 seconds, with streamlines of the external flow from PIV analysis (droplet reference frame). Data from double channel fluorescence microscopy, with illumination at $561 \mathrm{~nm}$ (Nile Red doped oil, red emission) and $488 \mathrm{~nm}$ (tracer colloids, green emission). (b) Sketch of the filling and growth of micelles travelling in a boundary layer along the interface, causing a propulsive Marangoni flow. (c) Microscopy set-up schematic with the droplet (radius $30 \mu \mathrm{m}$ ) swimming in a Hele-Shaw cell (height $60 \mu \mathrm{m}$ ). (d) Sample micrograph, with the droplet's centroid trajectory traced in white. 


\subsection{Simultaneous visualisation of chemical and hy- drodynamic fields}

To visualise the chemical and hydrodynamic fields involved in the droplet activity, we directly imaged the chemical field of swollen micelles by adding the hydrophobic dye Nile Red to the oil phase (Fig. 3.1 c,d, see also Appendix 3.9 and Video S1). The dye co-migrates with the oil molecules into the filled micelles, which fluoresce when illuminated. We seeded the surrounding medium, a supramicellar aqueous surfactant solution, with green fluorescent tracer colloids and measured the flow field using particle image velocimetry (PIV). The emission spectra of dye and colloids are sufficiently non-overlapping to be separately detected in dual channel fluorescence microscopy. Consequently, both fields can be simultaneously observed and analysed; we provide an example micrograph with an overlay of the extracted droplet trajectory in Fig. $3.1 \mathrm{~d}$. Due to the large size $(\sim 5 \mathrm{~nm})$ of the filled micelles, the time scale of their diffusive relaxation exceeds that of the droplet motion; thus, there is a persistent fluorescent trail in the wake of the droplet.

\subsection{Destabilised motion with increasing Péclet num- ber}

We begin, however, with an overview of the droplet dynamics using trajectory plots and statistical analyses of speed and orientational persistence taken from bright-field microscopy (Fig. 3.2). With increasing Pe, the droplet propulsion changes from uniform speeds and persistent motion to unsteady motion with abrupt reorientations (Fig. 3.2 a-d). We define $P(|\delta \theta(t)|)$ as the distribution of the reorientation angle $\delta \theta$ of the 2D droplet velocity $\boldsymbol{V}(t)$ during a fixed time step $\delta t$ [153],

$$
\delta \theta(t)=\arctan \left(\frac{\boldsymbol{V}(t) \times \boldsymbol{V}(t+\delta t)}{\boldsymbol{V}(t) \cdot \boldsymbol{V}(t+\delta t)}\right) .
$$

$P(|\delta \theta(t)|)$ broadens significantly, corresponding to more frequent and sharper reorientation events (Fig. 3.2 e). The faster decay of the angular velocity autocorrelation function,

$$
C_{V V}(t)=\left\langle\frac{\boldsymbol{V}\left(t_{0}+t\right) \cdot \boldsymbol{V}\left(t_{0}\right)}{\left|\boldsymbol{V}\left(t_{0}+t\right)\right|\left|\boldsymbol{V}\left(t_{0}\right)\right|}\right\rangle_{t_{0}},
$$




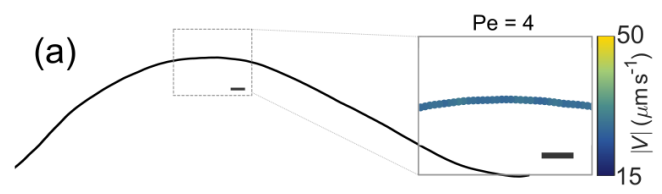

(b)

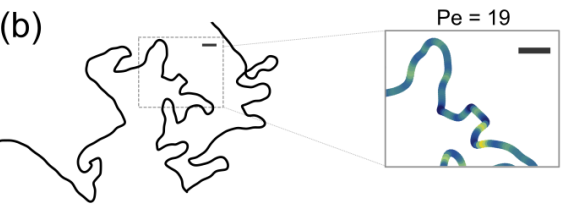

(c)

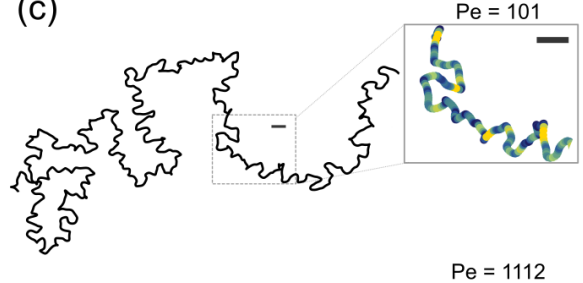

(d)

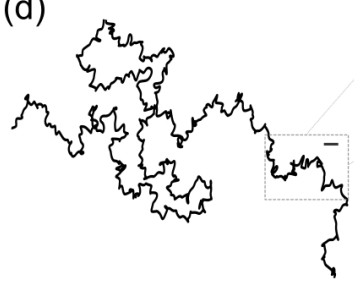

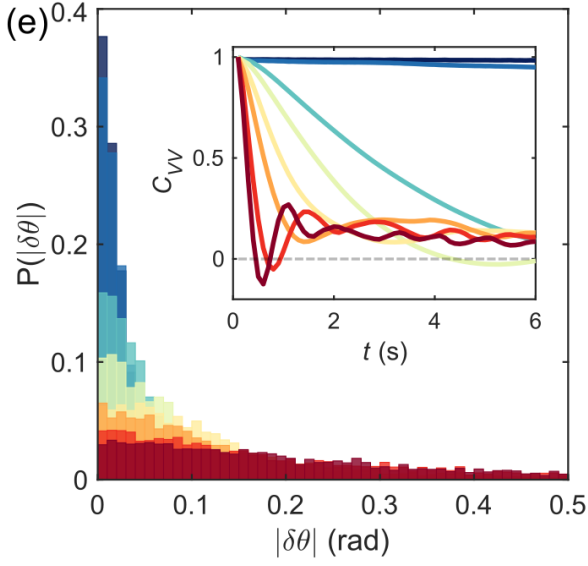

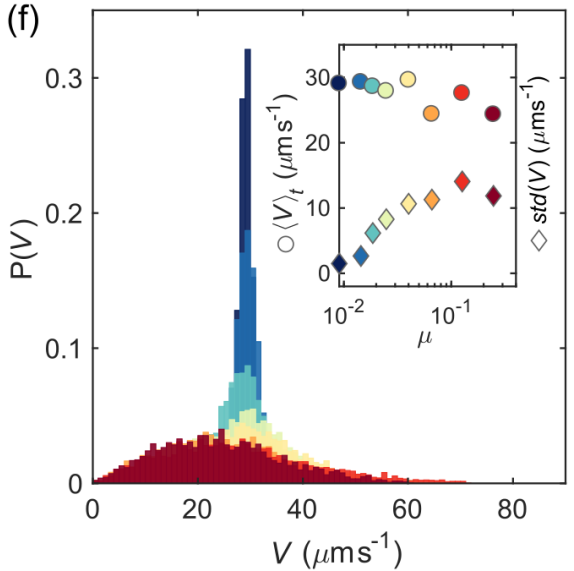

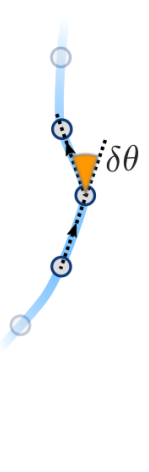

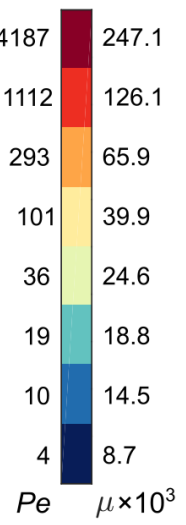

Figure 3.2 - Destabilized droplet motion with increasing Péclet number Pe. (a-d) Example trajectories of droplets for $P e \in\{4,19,101,1112\}$, with zoomed-in insets colourcoded by propulsion speed $V$. All scale bars are $50 \mu \mathrm{m}$. (e) Distribution of the velocity reorientation angle, $|\delta \theta|$ for increasing $P e$, measured during a time step $\delta t=0.1 \mathrm{~s}$ set by the video recording rate of $10 \mathrm{~Hz}$. Profiles of the velocity auto-correlation function, $C_{V V}$, in the inset, show the loss of directionality in swimming. (f) Distribution of propulsion speeds $V$ for increasing $P e$, with mean and standard deviation of speeds in the inset. See also Supporting Videos S2-S5. The color bar relating experimental Pe estimates to the viscosity ratio $\mu=\mu^{o} / \mu^{i}$ applies to all subsequent figures. 
illustrates the loss of directionality with increasing $P e$ (Fig. 3.2 e, inset). Fig. $3.2 \mathrm{f}$ shows that at sufficiently large $P e$, the speed distribution $P(V)$ includes values as small as zero (stopping events) and, surprisingly, as large as $70 \mu \mathrm{m} / \mathrm{s}$, much greater than the uniform speed of $30 \mu \mathrm{m} / \mathrm{s}$ observed for low $P e \approx 4$. While the mean speed barely changes with $P e$, the standard deviation of $V$ grows by over one order of magnitude (Fig. $3.2 \mathrm{f}$, inset). Hence, both the rotational and the translational motion of the swimmer are destabilised with increasing $P e$, similar to recent numerical studies of solid phoretic particles [154]. Note that the thermal fluctuations $\left(O\left(k_{b} T / 2 R_{d}\right) \sim 10^{-16} \mathrm{~N}\right)$ are negligible compared to the hydrodynamic drag force $\left(O\left(6 \pi \mu^{o} R_{d} V\right) \gtrsim 10^{-10} \mathrm{~N}\right)$, such that thermal noise is an unlikely cause for the unsteady swimming.
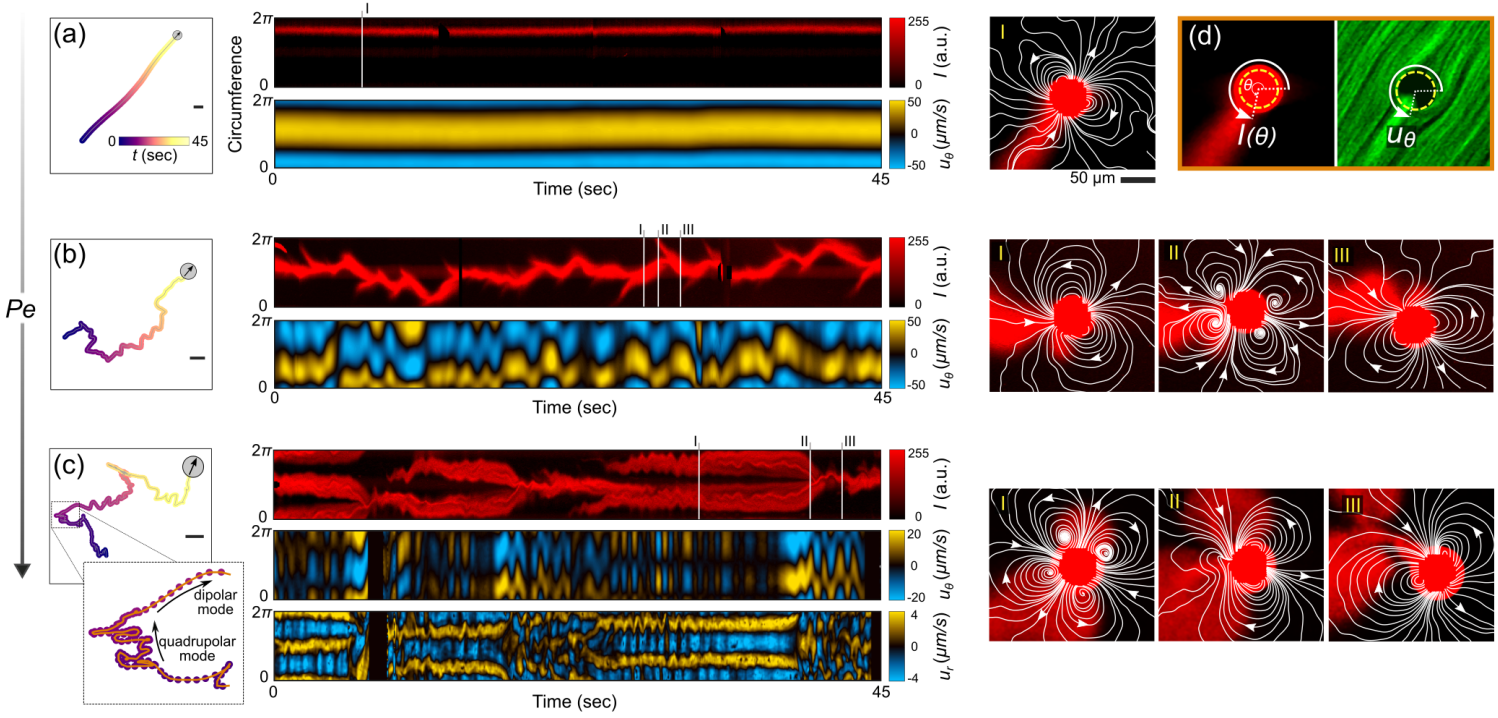

Figure 3.3 - Signatures of unsteady dynamics in the time evolution of chemical and hydrodynamic fields. Rows (a), (b) and (c) correspond to $P e \approx 4, P e \approx 36$ and $P e \approx 293$, respectively; Left column, trajectories colour coded by time; middle column, kymographs of $I$ and $u_{\theta}$ during 45 seconds of propulsion; right column, selected red channel images, overlaid by the flow streamlines at the laboratory reference frame. Each frame corresponds to the point in time indicated on the kymographs by I, II or III. Panel (d) defines the mapping of the profiles of red light intensity I (filled micelle concentration) and tangential velocity, $u_{\theta}$, around the droplet circumference onto the $y$ axis of the kymographs in the middle column. All $u_{\theta}$ profiles are in the translational droplet reference frame, but with $\theta=0$ fixed at the laboratory $x$ direction to visualize the reorientation dynamics. In (c), the third kymograph corresponds to the radial velocity $u_{r}$ in the laboratory reference frame to better depict the quadrupolar symmetry of the flow field. The second hydrodynamic mode starts to appear at intermediate Pe and dominates the dynamics for high Pe. See also Appendix 3.9, Fig. 3.11 for additional flow field examples. All scale bars are $50 \mu \mathrm{m}$. 


\subsection{Signatures of unsteady dynamics in the time evolution of chemical and hydrodynamic fields}

To investigate the origin of this unsteady behaviour, we studied the evolution of chemical and hydrodynamic fields around the droplet. We extracted the tangential flow velocity $u_{\theta}(\theta)$ and the red fluorescence intensity $I(\theta)$ of the chemical field close to the interface (Fig. $3.3 \mathrm{~d}$, Appendix 3.9), and mapped them in kymographs $I(\theta, t)$ and $u_{\theta}(\theta, t)$.

For low $P e \approx 4$, at persistent propulsion, $I(\theta, t)$ shows a single fixed-orientation band marking the origin of the filled micelle trail at the rear stagnation point of the droplet (Fig. 3.3 a and Video S6). The two bands in $u_{\theta}(\theta, t)$ correspond to a steady flow field with dipolar symmetry that is consistent with the $I(\theta, t)$ profile. On the right side of Fig. 3.3 a we have superimposed the streamlines of this dipolar flow field on the corresponding chemical micrograph at the time marked by I in the $I(\theta, t)$ kymograph.

For intermediate $P e \approx 36$ (Fig. $3.3 \mathrm{~b}$, Video $\mathrm{S} 7$ ), $I(\theta, t)$ shows secondary branches forming at the anterior stagnation point of the droplet and subsequently merging with the main filled micelle trail. This coincides with a transient second hydrodynamic mode with quadrupolar symmetry (Fig. $3.3 \mathrm{~b}, \mathrm{II}$ ), causing the accumulation of an additional aggregate of filled micelles at the droplet anterior (see also Appendix 3.9, Fig. 3.11 for additional flow field examples).

The ratio of the diffusive $\left(R_{d}^{2} / D_{f m}\right)$ to advective $\left(R_{d} / V\right)$ time scales for the migration of filled micelles is $\frac{V R_{d}}{D_{f m}} \gg 1$ for all experiments, assuming a diffusion coefficient $D_{f m}=k_{B} T / 6 \pi \mu^{o} r_{f m}$, with a micellar radius of $O\left(r_{f m}\right) \sim 2.5 \mathrm{~nm}$. Therefore, the aggregate is unlikely to dissipate by diffusion, and will continue to grow as long as the quadrupolar mode exists. However, this mode is not stable. Eventually, the dipolar mode dominates and advects the secondary aggregate towards the main trail (Fig. $3.3 \mathrm{~b}, \mathrm{III}$ ). The transport of the aggregate along one side of the droplet locally disturbs the interfacial flow, leading to an abrupt reorientation of the swimming direction (Fig. 3.3 b,I-III). As shown in the trajectories in Fig. $3.2 \mathrm{~b}$ and c, these reorientation events become more frequent with increasing $P e$; accordingly, $u_{\theta}$ in Fig. $3.3 \mathrm{~b}$ exhibits quasi-periodic reorientation patterns.

For high $P e \approx 293$ (Fig. 3.3 c, Video S8), the quadrupolar mode eventually prevails, resulting in a predominantly symmetric extensile flow around the droplet (Fig. $3.3 \mathrm{c}, \mathrm{I}$ ), as shown by a pronounced fourfold pattern in the additional kymograph $u_{r}(\theta, t)$ of the radial velocity. Due to the non-propelling quadrupolar mode the droplet is trapped in 
3.5 Signatures of unsteady dynamics in the time evolution of chemical and hydrodynamic fields
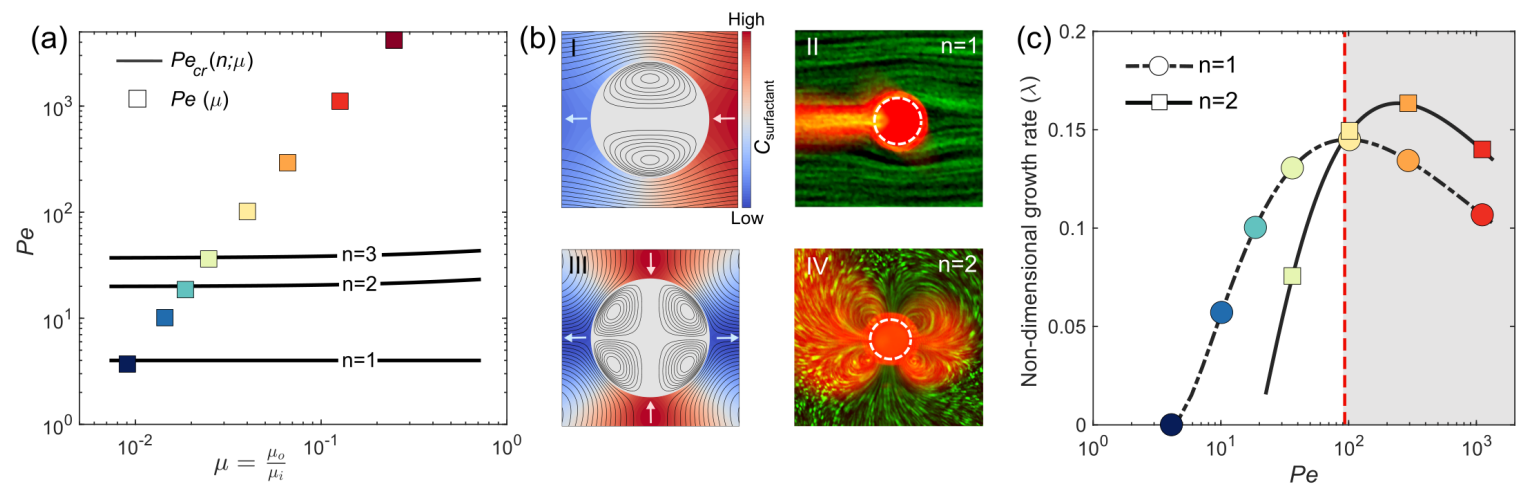

Figure 3.4 - Dependence of hydrodynamic modes on the Péclet number. (a) Critical Péclet $P e_{c r}$ values (black lines), necessary for the onset of different hydrodynamic modes $(n)$, with varying $\mu$; The markers $(\square)$ show the Péclet number Pe (eqn. (3.1)) which increases with $\mu$. The colour code is taken from Fig. 3.2 (b) (top) Steady self-propulsion of the active droplet; theoretical solution for $n=1$ mode (left) and experimental streak image for low Pe (right); (bottom) the extensile flow corresponding to $n=2$ mode (left) and the experimental image for higher Pe (right). The theoretical and the experimental flow fields are in the swimmer reference frame. (c) Instability growth rates corresponding to the first two hydrodynamic modes as a function of Pe. Beyond the dashed vertical line (grey region) $\lambda_{n=2}>\lambda_{n=1}$ and thus the $n=2$ mode is dominant.

place. The gradual accumulation of filled micelles at the two stagnation points with radially outward flow manifests in two stable branches in the chemical kymograph (marked by I in Fig. 3.3c). The growth of the two micellar aggregates locally generates a lateral chemical gradient, which eventually pushes the droplet out of its self-made trap. Concomitantly, the two points of filled micelle emission move along the droplet interface and merge on the new rear side of the droplet into a single filled micelle trail (Fig. 3.3 c,II and III). The chemorepulsion from the local field micelle gradient induces an apparent dipolar mode which gradually decays as the droplet leaves the self-made trap. Now, the quadrupolar mode re-saturates, with an aggregate growing at the droplet anterior, until the droplet is trapped again and a new bimodal 'stopand-go' cycle begins. Since the escape direction is always lateral, consecutive runs are approximately perpendicular, resulting in the sharp reorientation events apparent in the trajectories in Fig. $3.3 \mathrm{c}$ and Fig. $3.2 \mathrm{~d}$, as well as the broadening $|\delta \theta|$ distribution in Fig. 3.2 e. 


\subsection{Dependence of hydrodynamic modes on the Pé- clet number}

In order to understand the dependence of the emergence of bimodal motility on $P e$, we analysed the underlying advection-diffusion problem for the active droplet within the framework of an axisymmetric Stokes flow following refs. [54, 57, 73, 155] (see Fig. 3.4, and Appendix 3.9). At the smallest value of $\mu, P e$ is approximately equal to the critical value of 4 necessary for the onset of the first hydrodynamic mode $(n=1)$, i.e. the mode with dipolar flow symmetry [54, 57, 73]. With increasing $\mu, P e$ (markers in Fig. 3.4a) eventually exceeds the critical values necessary for the onset of the higher hydrodynamic modes (lines in Fig. $3.4 \mathrm{a}$ ), specifically the second hydrodynamic mode $(n=2)$, i.e. the mode with quadrupolar symmetry. A linear stability analysis around an isotropic, quiescent base state (see Appendix 3.9 and [57, 73]), which is the idealized starting point for each experiment, shows that for small to moderate $P e$, the non-dimensionalised instability growth rate $\lambda$ for $n=1$ exceeds that for $n=2$ (Fig. 3.4c). Accordingly, for lower $P e, n=1$ dominates, resulting in steady self-propulsion stemming from the fore-aft asymmetry of the surfactant distribution (Fig. 3.4 b,I). Consequently, the active droplet exhibits persistent steady translation (trajectories in Fig. $3.2 \mathrm{a}, \mathrm{b}$ ) with a dominant dipolar flow field (Fig. 3.4 b,II and Fig. $3.3 \mathrm{a}$ ). However, for $P e \gtrsim 92, n=2$ (Fig. 3.4 b,III) has a faster instability growth rate (Fig. 3.4c), thereby becoming the dominant mode when evolving from the quiescent base state. Accordingly, the droplet is initially stuck in a non-propelling mode with a quadrupolar flow field (similar to Fig. 3.4 b,IV). Such quadrupolar flow field gives rise to the filled micelle field with the two points of outflux. The synergy between the $n=2$ mode and the transiently-growing filled micelle field subsequently results in the onset of the bimodal 'stop-and-go' motion of the droplet for moderate to higher $P e$ (trajectories in Fig. 3.2 c,d). Since we observe in experiments with $P e \gtrsim 100$ that the active droplet experiences sustained periods of dynamical arrest during which it remains stationary with a surrounding extensile flow (Fig. $3.3 \mathrm{c}$ ), it appears that the $n=2$ mode can also evolve from a non-quiescent state and prevail in a similar Péclet regime as derived from the performed stability analysis. Note that we restrict our analysis to the first two hydrodynamic modes since these two are solely responsible for the droplet propulsion and the associated far-field hydrodynamic disturbance. 

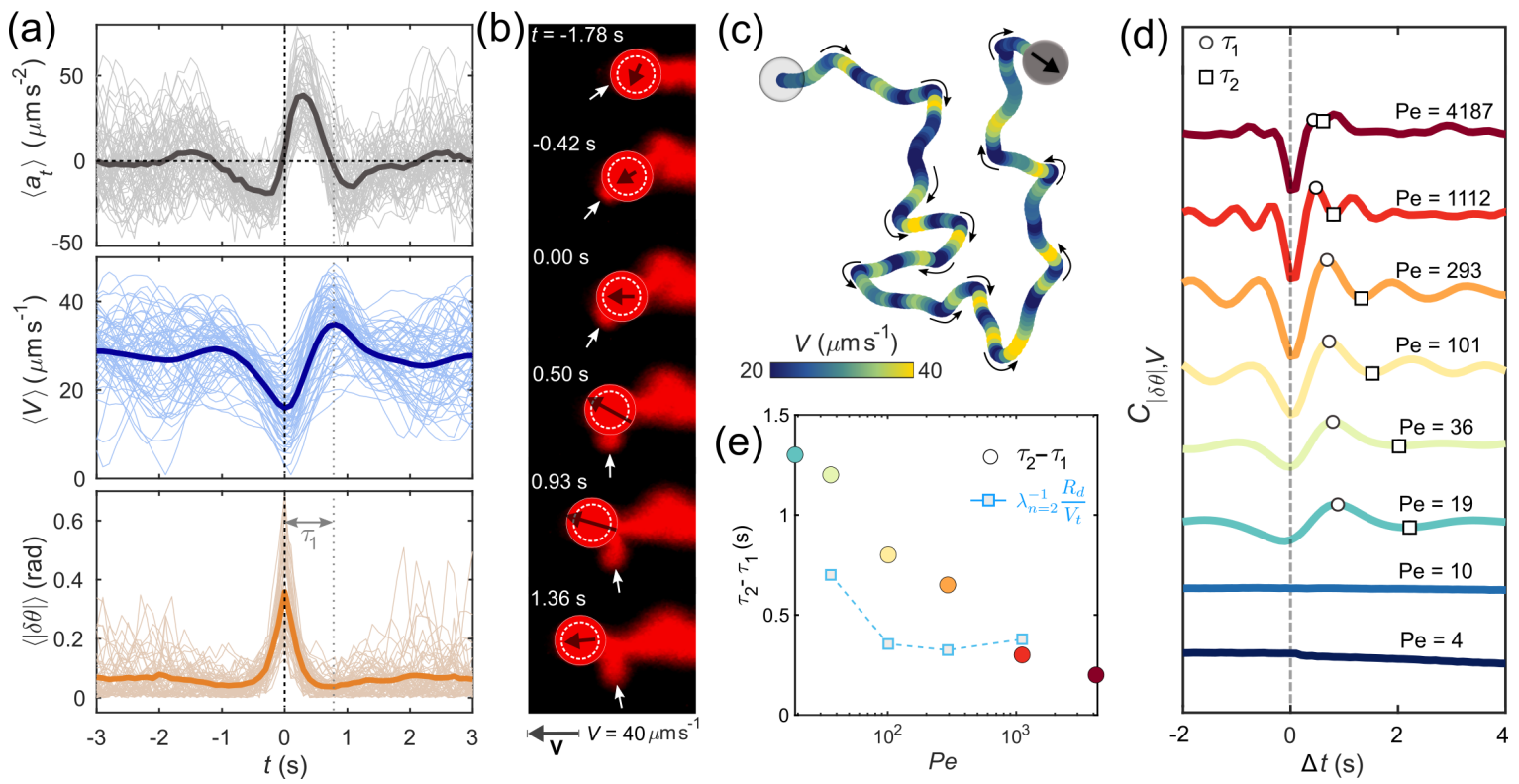

Figure 3.5 - Interactions with self-generated chemical gradients cause speed bursts at reorientation events. (a) Conditional averaging of tangential acceleration, $a_{t}$, speed, $V$, and reorientation angle, $|\delta \theta|$, for abrupt reorientation events at $P e \approx 36$ (see Appendix 3.9, Fig. 3.12, for an illustration of the identification criteria). The dotted line marks the maximum speed at $t=\tau_{1}$ after reorientation. (b) Video stills of the chemical field for one such event with $t=0 \mathrm{~s}$ set to the point of minimum speed; white arrows track the accumulation of the secondary filled micelle aggregate at the anterior stagnation point and its advection along the interface, black arrows correspond to the droplet velocity vector. The droplet speed is maximal when the secondary aggregate and the trail merge at $t=0.93 \mathrm{~s}$. See also Videos S9 and S10. (c) An example trajectory for $P e \approx 36$. Any reorientation event (curved arrows) is preceded by a deceleration and followed by an acceleration. The lowest speed occurs at the point with the highest curvature. (d) Correlation function between reorientation angle and speed, $C_{|\delta \theta|, V}(\Delta t)$ for increasing $P e$. Times $\tau_{1}$ and $\tau_{2}$ (next reorientation event) are identified by the respective peak and dip in $C_{|\delta \theta|, V}$. (e) Time scale for the growth of the $n=2$ mode vs. corresponding $P e$ : experimentally obtained, $\tau_{2}-\tau_{1}$ (o), compared to values from stability analysis, $\lambda_{n=2}^{-1} R_{d} / V_{t}(\square)$. 


\subsection{Interactions with self-generated chemical gra- dients cause speed bursts}

It remains to explain the broadening of $P(V)$ with increasing $P e$ (Fig. 3.2 e), particularly the remarkable bursts in speed for high $P e$. While the dipolar mode is propulsive, the quadrupolar mode is not. Hence, the growth and decay of the respective modes will affect the droplet speed. As shown in Fig. 3.3, recurrent transitions between the two hydrodynamic modes lead to abrupt reorientation events; we therefore investigated the correlation between changes in speed and reorientation angle $|\delta \theta|$.

In a typical trajectory for intermediate $P e \approx 36$, each sharp turn is preceded by a deceleration and followed by an acceleration, as shown in the plot of the positional data colour-coded by speed in Fig. 3.5 c. Signatures of these correlations in the droplet dynamics appear in the conditional averages

$$
\left\langle X\left(t-t_{i}\right)|| \delta \theta\left(t_{i}\right) \mid>0.2, \frac{\mathrm{d} \delta \theta\left(t_{i}\right)}{\mathrm{d} t}=0\right\rangle_{i}
$$

of $|\delta \theta|, V$ and tangential acceleration $a_{t}$ as quantities $X$ for all sharp reorientation events $i$ in the trajectory, centered at $t=t_{i}$ of maximum $|\delta \theta|$ (Fig. $3.5 \mathrm{a}$ ); the events were identified by choosing a threshold value of $|\delta \theta|>0.2$ (see Appendix 3.9, Fig. 3.12 ).

We can now directly compare these dynamics to the higher resolution fluorescence data taken at $P e \approx 36$ presented in the kymographs in Fig. 3.3 b. Fig. 3.5 b shows a series of micrographs of the chemical field, with arrows marking the droplet velocity vector (black) and the position of the secondary filled micelle aggregate (white). The aggregate accumulates, is then entrained and finally merges with the posterior trail, corresponding to the creation and merging of a secondary chemical branch in the kymograph.

For $t<0$ the droplet decelerates while the secondary aggregate is accumulating. $t=0$ marks the point in time where $V$ is minimal and the aggregate is on the cusp of leaving the anterior stagnation point. For $t>0$, the aggregate is advected to the droplet posterior and the droplet accelerates due to the re-saturation of the dipolar mode. $V$ peaks once the aggregate has merged with the main trail — creating an amplified fore-aft gradient - at $t \approx 1 \mathrm{~s}$, which is comparable to the advective timescale $R_{d} / V \approx 1 \mathrm{~s}$. In the wide-field data analysis in Fig. $3.5 \mathrm{a}$, this is the time $\tau_{1}$ it takes the droplet to reach maximum speed after a reorientation. 


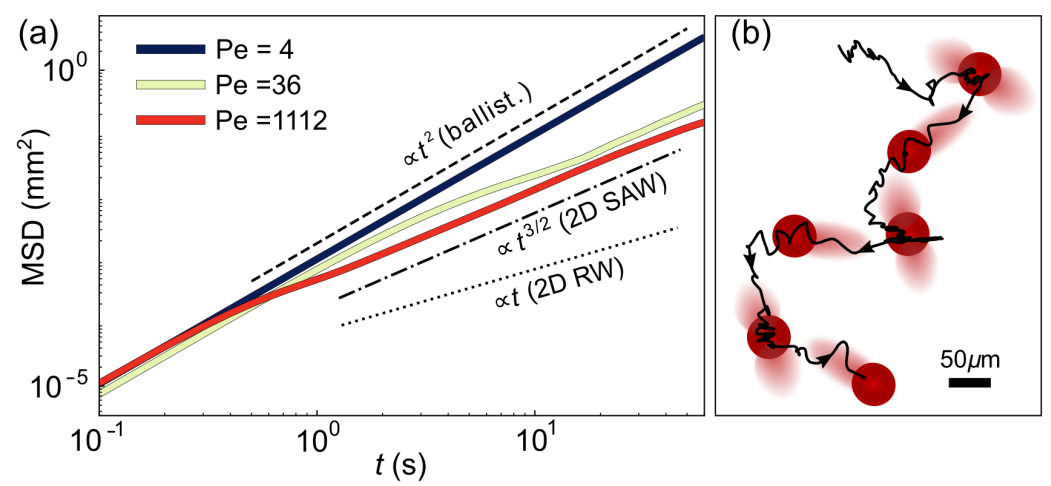

Figure 3.6 - Anomalous diffusive swimming. (a) Mean squared displacement profiles of experimental trajectories for different Pe. Dashed lines mark the predicted scaling for ballistic motion, $\propto t^{2}, 2 D$ self-avoiding walk $(S A W), \propto t^{3 / 2}$, and random walk $(R W)$, $\propto t$. For higher Pe, there is a transition from ballistic to $2 D S A W$. (b) A segment of the trajectory associated with the $S A W$ and schematics of the droplet exhibiting bimodal swimming causing the SAW. See also Appendix 3.9, Fig. 3.13.

We now use the correlation function between $V$ and $|\delta \theta|$,

$$
C_{|\delta \theta|, V}(\Delta t)=\langle|\delta \theta(t)| \cdot V(t+\Delta t)\rangle_{t}
$$

plotted in Fig. 3.5 d, to estimate the growth times of the second mode from our data for $P e>10$. Since $V$ is minimal at maximum $|\delta \theta(t)|$ (Fig. $3.5 \mathrm{~d}), C_{|\delta \theta|, V}(\Delta t)$ dips at $\Delta t=0$. It subsequently peaks at the point of maximum $V$ with a time delay $\Delta t=\tau_{1}$, when the contribution of the propulsive dipolar flow is maximal. The next dip at a time $\tau_{2}>\tau_{1}$ marks the next reorientation event; based on the discussion pertaining to Fig. 3.3 and Fig. $3.4 \mathrm{c}$, for moderate to high $P e, \tau_{2}-\tau_{1}$ approximately corresponds to the time scale for the growth and re-saturation of the $n=2$ mode during the bimodal motility (i.e. starting from a non-quiescent base state). Nevertheless, we compare this experimentally obtained $\tau_{2}-\tau_{1}$ with the theoretical growth times for the $n=2$ mode starting from the isotropic base state, $\lambda_{n=2}^{-1} R_{d} / V_{t}$ (Fig. 3.4c), for different values of $P e$. Fig. 3.5 e shows that these two time scales, which are strictly speaking different, still are of the same order of magnitude and show similar decreasing trend with increasing $P e$. We note that the growth time of the dipolar flow above $P e \approx 100$ cannot be used for comparison to $\lambda_{n=1}$, since this flow is imposed by the lateral chemical gradient. However, we can assume that this gradient increases with $P e$, resulting in faster acceleration, markedly higher swimming speeds, and hence, reduced $\tau_{1}$, as observed experimentally (Fig. $3.5 \mathrm{~d}$ ). 


\subsection{Consequences for spatial exploration}

Reminiscent of gait switching dynamics in biological locomotion, we have demonstrated the emergence of complex swimming behaviour in a minimal active droplet system by tuning the Péclet number. We found a transition from persistent swimming at low $P e$ to chaotic bimodal swimming at high $P e$ - the latter results from the excitation of higher hydrodynamic modes beyond critical $P e$ values, while the continuous switching between them is caused by the self-generated chemical gradient in the environment.

This gradient sensitivity causes trail avoidance [89], which in turn affects the way these droplet swimmers explore their environment. With increasing reorientation frequency, we find a transition from quasi-ballistic propulsion to a $2 \mathrm{D}$ self-avoiding walk (2D SAW). This effect is illustrated by the trajectories in Fig. $3.2 \mathrm{a}-\mathrm{d}$, and also by the fact that $C_{V V}$ in Fig. $3.2 \mathrm{e}$ does not decay to zero. For a statistical analysis we have plotted mean squared displacements for selected $P e$ values in Fig. 3.6 a, which reproduce the expected scaling with $t^{2}$ (ballistic) for $P e \approx 4$ and a transition to $t^{3 / 2}$ (2D SAW, [156]) for $P e \gtrsim 36$, with the crossover time decreasing with increasing $P e$. While transitions to random walks governed by run-and-tumble gait switching are common in bioswimmers [157], self-avoidance requires chemical self-interaction [158].

Examples of anomalous diffusion driven by repulsive biochemical signalling have been found in the spreading of slime molds [159, 160] — active droplets can show analogous behaviour based on purely physicochemical mechanisms.

\section{Conclusion}

In this work, we demonstrated that the manner in which hydrodynamic and selfgenerated chemical fields are coupled determines the nonlinear dynamics of autophoretic micro-swimmers. The fluorescence-based visualisation technique used to simultaneously probe this coupling can provide insight into many recent autophoretic models [57, $58,67,101,152,161,162]$. For example, extensive theoretical studies [163-166] have demonstrated the importance of quantifying far-field and near-field contributions, coupling to chemical fields and the effects of confinement to understand how swimmers approach each other or form bound states, which is vital to nutrient entrainment, food uptake and mating in bioswimmers.

While many micro-swimmer models incorporate unsteady dynamics via stochastic fluctuations, we have shown that the interplay of nonlinear dynamics and interaction 
with the history of motion also allows for the emergence of memory-driven chaotic behaviour. An appealing example from a different field are droplet walkers on a vibrated bath [167], which show a transition from persistent to a bimodal, stop-and-go motion based on an effective 'system memory' parameter [168, 169]. The corresponding theoretical framework [168] is general enough to also apply to bimodal chaotic motion in droplet swimmers.

We acknowledge fruitful discussions with Stephan Herminghaus, Arnold Mathijssen and Prashanth Ramesh, as well as financial and organisational support from the DFG SPP1726 "Microswimmers" (CCM, RD, BVH), the ERC-Advanced Grant "DDD" (DL, $\mathrm{MJ})$, and the Max Planck Center for Complex Fluid Dynamics. 


\subsection{Appendix}

\section{Materials and Methodology}

\section{Materials and characterisation}

Our samples consisted of droplets of (S)-4-Cyano-4'-(2-methylbutyl)biphenyl (CB15) doped with the fluorescent dye Nile Red in an aqueous solution of the anionic surfactant tetradecyltrimethylammonium bromide (TTAB) corresponding to $5 \mathrm{wt} . \%$ in pure water, with a critical micelle concentration of $C M C=0.13 \mathrm{wt} . \%$ (50 $\mathrm{mg}$ in $1 \mathrm{ml}$ of solution). We purchased CB15, TTAB, and Nile Red from commercial suppliers (Synthon Chemicals and Sigma-Aldrich) and used them as is. We controlled the viscosity of the swimming medium, $\mu_{o}$, by adding glycerol to the aqueous TTAB solution.

We used an Anton Paar MCR 502 rotational rheometer to characterise the shear viscosity of water-glycerol-surfactant solutions (Fig. 3.7). Experiments were carried out using a cone-plate geometry, to find shear-rate versus shear-stress curves at a fixed temperature, and viscosity versus temperature a fixed shear rate. To limit effects of solution evaporation, the cone-plate geometry was surrounded by a water bath and covered by a Peltier hood. Over the shear rate range $0.01 \mathrm{~s}^{-1}<\dot{\gamma}<100 \mathrm{~s}^{-1}$, viscosity was found to be constant, such that our solutions are well-described as Newtonian, as should be expected: Water/glycerol mixtures are used as Newtonian standard media throughout the existing literature.

To estimate the surfactant consumption rate $q_{s}$ in (3.1), we extracted the droplet shrinking rate $\mathrm{d} R_{d} / \mathrm{d} t$ from the bright field microvideography data presented in Fig. 3.2 . We found a moderate dependence on the glycerol fraction (Fig. 3.8), which we included as a first order approximation, via linear regression (blue line), to evaluate $q_{s}$ in the $P e$ estimates in the main manuscript.

\section{PDMS soft lithography for droplet generation}

For the production of monodisperse oil droplets, we fabricated microfluidic channels inhouse, using standard soft lithography techniques. First, 2D photomasks were designed in AutoCad, and then printed onto an emulsion film in high-resolution (128,000 dpi) by a commercial supplier (JD Photo-Tools). Next, the photoresist SU-8 3025 (MicroChem) was spin-coated onto a 4 inch diameter silicon wafer (Si-Mat), where spin-speed and duration were adjusted to give a controllable uniform thickness. A negative mold was cured in the SU-8 through the photomask by UV light exposure. After further 


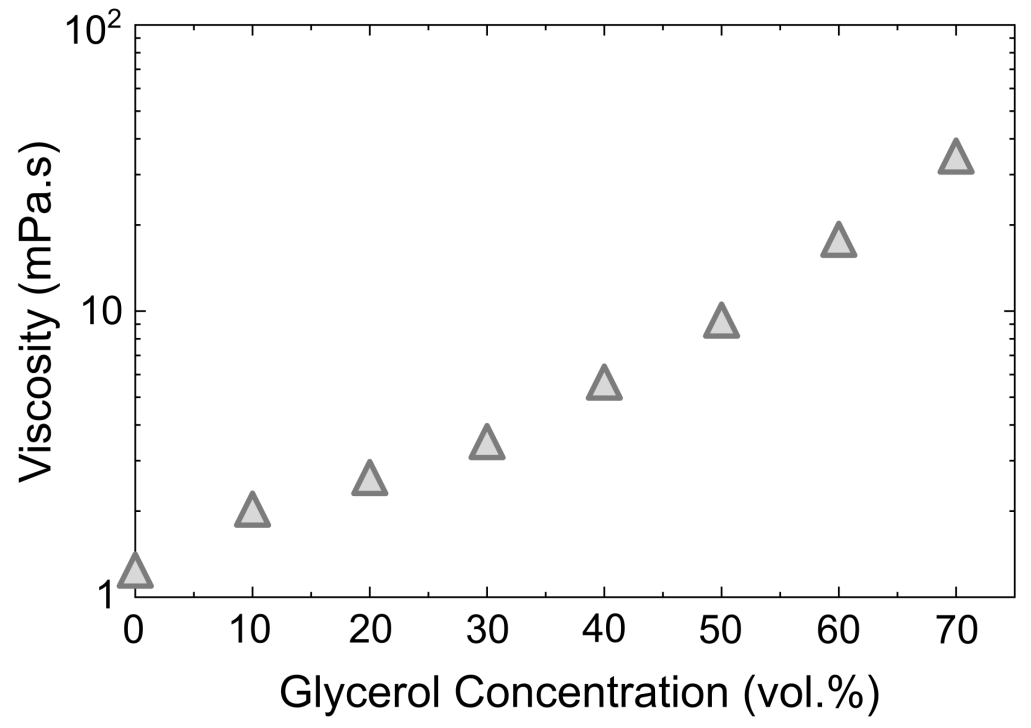

Figure 3.7 - Viscosity of the swimming medium, a mixture of water, glycerol and TTAB surfactant, for increasing glycerol/water ratios. Surfactant concentration is $50 \mathrm{mg}$ in $1 \mathrm{ml}$ of solution.

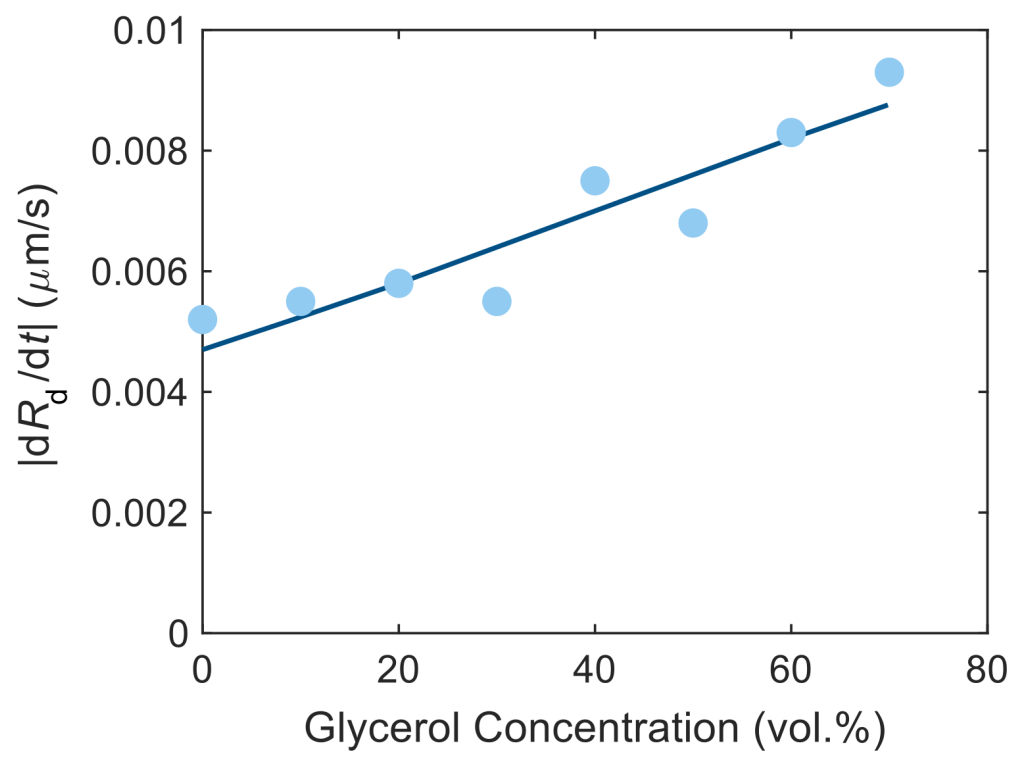

Figure 3.8 - Solubilisation rate $\left|\mathrm{d} R_{d} / \mathrm{d} t\right|$, for increasing glycerol/water ratios, blue line marks a linear regression fit to $y=0.000058 x+0.0047$. Surfactant concentration is $50 \mathrm{mg}$ in $1 \mathrm{ml}$ of solution. 


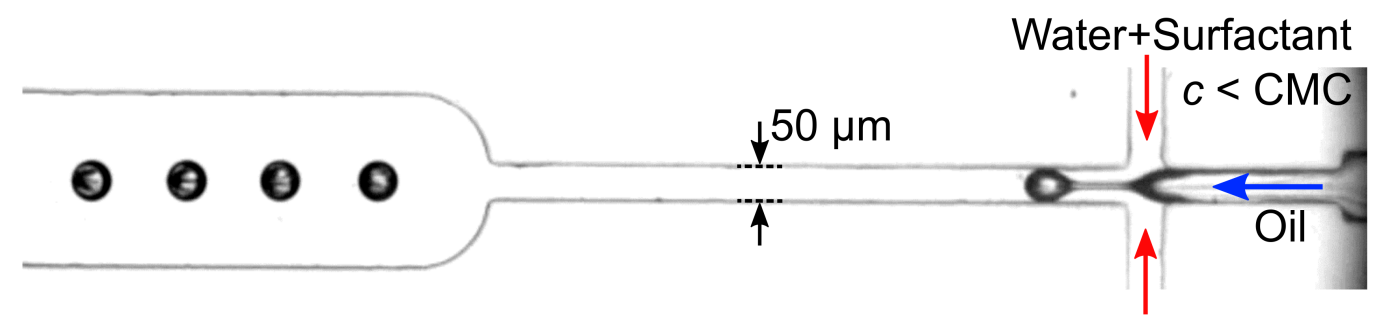

Figure 3.9 - The microfluidic chip used to produce mono-dispersed oil droplets in a surfactant solution.

chemical treatment with photoresist developer, uncured SU-8 was removed, leaving behind cured SU-8 microstructures on the silicon wafer.

We then poured a poly(dimethyl siloxane) (PDMS, Sylgard 184, Dow Corning) mixture of 10:1 volumetric ratio of base to cross-linker over the wafer, and baked for 2 hours at $80^{\circ} \mathrm{C}$, producing a solid PDMS layer with microstructured indentations. We peeled the indented PDMS from the wafer, and punched holes through it to create liquid inlets/outlets at opposing ends of the channels. The structured PDMS surface, as well as a glass coverslip, were cleaned and treated with partial pressure air-plasma (Pico P100-8; Diener Electronic GmbH + Co. KG) for 30 seconds, and then pressed together, bonding the two surfaces. Fig. 3.9 shows a micrograph of such a PDMS chip during droplet production.

The walls of these microfluidic chips were selectively treated to hydrophilise the channels where surfactant solution will flow. This prevents oil from wetting the walls during droplet production. We followed the technique of Petit et al.[106]: First, the channel walls were oxidised by a 1:1 mixture of hydrogen peroxide solution (H2O2 at 30 wt.\%, Sigma-Aldrich) and hydrochloric acid ( $\mathrm{HCl}$ at 37 wt.\%, Sigma-Aldrich). This mixture was flushed through the channels for approximately 2 minutes by using a vacuum pump system. After the oxidation, the channel was rinsed by flushing double distilled water for 30 seconds. Next, a 5 wt. $\%$ solution of the positive polyelectrolyte poly(diallyldimethylammonium chloride) (PDADMAC, Sigma-Aldrich) was flushed for 2 minutes through the oxidised channel of the device. The PDADMAC binds to the activated channel walls by ionic interactions. Finally, a $2 \mathrm{wt} . \%$ solution of the negative polyelectrolyte poly(sodium 4- styrenesulfonate) (PSS, Sigma-Aldrich) was flushed for 2 minutes. 


\section{Droplet generation}

Once the chips had been treated, we mounted syringes of oil and $0.1 \mathrm{wt} . \%$ aqueous TTAB solution to a microprecision syringe pump (NEM-B101-02B; Cetoni GmbH), and connected these to the two inlets of the microfluidic chip via Teflon tubing (39241; Novodirect $\mathrm{GmbH}$ ), and tuned the flow speed through the chip until the desired droplet size was reached. Once droplet production was monodisperse (after approximately 5 minutes) and at a steady state, these droplets were collected in a bath of $0.1 \mathrm{wt} . \%$ TTAB solution. This solution is of a high enough concentration to stabilize the droplets against coalescence, but not high enough to induce solubilization.

\section{Fabrication of the observation Hele-Shaw cell}

The swimming behaviour of the droplets was observed in a quasi-2D Hele-Shaw reservoir, which we fabricated directly from SU-8 photoresist without PDMS casting. To fabricate the reservoirs we therefore used a photo-mask with inverted polarity. We spin-coated the photoresist directly onto a glass slide $\left(50 \times 75 \mathrm{~mm}^{2}\right)$ and followed the same procedure for photo-lithography as outlined in Section 3.9. This resulted in a layer of crosslinked SU-8 (thickness $\approx 60 \mu \mathrm{m}$ ) with reservoirs of the dimensions $8 \times 13 \mathrm{~mm}$. These reservoirs were filled with the samples, sealed with a glass cover slip and put under a microscope.

\section{Double-channel fluorescent microscopy technique}

We used double-channel fluorescent microscopy for simultaneous imaging of the chemical and hydrodynamic fields. A schematic of the setup is shown in Fig. 3.10. Two laser units excite the test section. The Nile Red dye (Thermo Fisher Scientific), which visualises the oil phase, is excited with a $561 \mathrm{~nm}$ laser and emits light at a maximum of $\sim 630 \mathrm{~nm}$. The green fluorescent particles (FluoSpheres ${ }^{\mathrm{TM}}$, yellow-green fluorescent, $500 \mathrm{~nm}$ in diameter), which visualise the fluid flow around the droplet, are excited with a $488 \mathrm{~nm}$ laser and emit light at a maximum of $\sim 510 \mathrm{~nm}$. The emitted light was separated using a beam splitter and appropriate filters for each emission maximum. We also used a spatial pinhole (confocal microscopy) to enhance image quality. Examples of snapshots recorded on each channel are shown in Fig. 3.10 b,c.

\section{Image processing and data analysis}

To observe the long time statistical behaviour of the active droplets, as in Fig. 3.2, we observed their motion in a glass-bounded Hele-Shaw cell (quasi-two dimensional 
Emergence of bimodal motility in active droplets
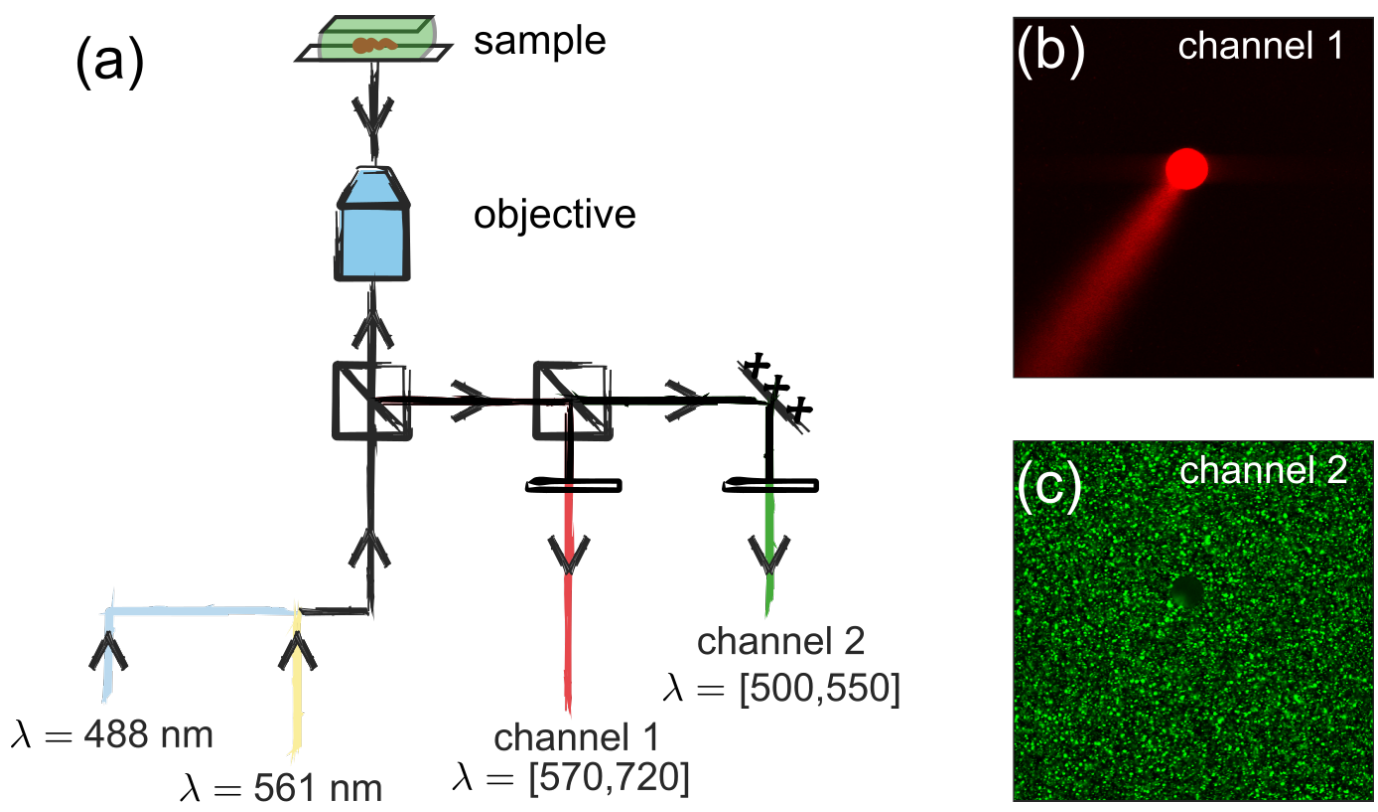

Figure 3.10 - Dual-channel fluorescent microscopy. (a) Light path schematic with excitation laser lines. $(b, c)$ Example micrographs showing the separated emission from filled micelles (b) and fluorescent tracers (c).

reservoir, $13 \times 8 \mathrm{~mm}$ and height $h \approx 60 \mu \mathrm{m}$ ) under a bright field microscope (Leica DM4000 B) at low magnification $(5 \times)$ compared to the double-channel fluorescence microscopy setup. Videos were recorded at a frame rate of $10 \mathrm{fps}$ using a Canon $($ EOS $600 d)$ digital camera $(1920 \times 1080 \mathrm{px})$. The droplet coordinates in each frame were extracted from video frames using the common Python libraries numpy, PIL and openCV (scripts available on request). Steps include background correction, binarisation, blob detection by contour analysis and minimum enclosing circle fits. Swimming trajectories were obtained using a frame-by-frame nearest-neighbour analysis.

To acquire the kymographs of the chemical field and tangential and radial velocities around the droplet interface, we observed the droplet behaviour by double-channel fluorescent microscopy as described in Section 3.9. We used a $512 \times 512$ pixels camera at a frame rate of $14 \mathrm{fps}$ connected to a $20 \times$ objective. First we split the red (NileRed, filled micelles) and green (tracer particles) channels. Then, the red frames were used to extract the droplet coordinates via the blob detection algorithm described above. We developed a MATLAB script that centred the droplet and recorded the red light intensity value along the interface at a distance $15.6 \mu \mathrm{m}$ for $P e \approx 4$ and 36 and $20.4 \mu \mathrm{m}$ for $P e \approx 293$. We note that it was not possible to record the intensity closer to the interface because the strong fluorescence from the large amounts of dye inside the droplet created a very bright region extending several micrometres beyond the actual 
interface. We plotted the extracted profiles versus time to generate spatiotemporal kymographs.

For a quantitative analysis of the flow field around the droplet we performed particle image velocimetry (PIV) on the tracer particles images (green channel) using the MATLAB-based PIVlab interface [133]. The objective was focused on the mid-plane of the Hele-Shaw cell. We defined a moving mask for the area covered by the droplet. We performed the analysis in $16 \times 16$ pixel interrogation windows with $75 \%$ overlap. The spatial resolution is $1.2 \mu \mathrm{m} / \mathrm{s}$. After obtaining the velocity vector field, we centered the droplet and read the velocity vectors at a certain distance from the droplet interface (3.6 $\mu \mathrm{m}$ for $P e \approx 4$ and 36 and $8.4 \mu m$ for $P e \approx 293)$. The tangential $\left(u_{\theta}\right.$, in the droplet reference frame) and radial ( $u_{r}$, only for $P e \approx 293$, in the lab reference frame) velocity components were then calculated and plotted in the kymographs. Due to the impermeability boundary condition, the radial component of the velocity directly at the interface is supposed to be zero; however, since we read the values at a certain distance from the interface there was an inward and outward radial contribution to the flow. We used this observation in particular at $P e \approx 293$ to show the quadrupolar symmetry of the flow field at the stopping moment.

In Fig. $3.1 \mathrm{a}$ and supplementary video S1, we tracked the droplet and centred it in the image. To obtain the pathlines of the tracer particles in the video we used FlowTrace [170] to convolve a superposition of 10 frames for each image. For Fig. 3.1 a we superimposed 30 frames. To visualise the motion of the tracer particles in Fig. 3.4 b,IV and the supplementary videos S6-S9, we processed the green channel of the input video ( 8 bit RGB) as follows: for each pixel coordinate, the intensity was replaced by its standard deviation within a 20 frame window around the current frame. Each frame was subsequently contrast maximised within a $[0,255]$ intensity range. The red and blue channels were not modified. This procedure was inspired by ImageJ's Z projection algorithm; the respective Python code is available on request.

\section{Viscosity dependence of hydrodynamic modes}

In this appendix we describe the mathematical framework for the coupled hydrodynamic and advection-diffusion problems pertaining to the active droplet system. Note that we have followed the solution methodology of refs. [54, 57, 73], and have reworked each step of the analysis for the present system. The appendix shows the origins of all expressions and equations (including the scaling analyses necessary for simplifications) needed to understand the theoretical framework, and importantly, the origin of Fig. 4. 
Emergence of bimodal motility in active droplets

We especially show each step of the linear stability analysis so that the derivation of the equations governing the instability growth rates for the hydrodynamic modes are clear.

\section{Governing equations and boundary conditions for the active droplet system}

Considering an axisymmetric Stokes flow (Reynolds no. for the swimming of the active droplet $R e \sim 10^{-4}$ ), and the impermeability of the droplet interface, the flow field around and inside the spherical active droplet (capillary number $\mathrm{Ca}<<1$ ) can be expressed in terms of the non-dimensional stream function $\psi$, in $(r, \theta)$ co-ordinate system, as $[54,73,155]$ :

$$
\begin{gathered}
\psi^{o}=a_{1}\left(\frac{1}{r}-r^{2}\right)\left(1-\eta^{2}\right) P_{1}^{\prime}(\eta)+\sum_{n=2}^{\infty} a_{n}\left(\frac{1-r^{2}}{r^{n}}\right)\left(1-\eta^{2}\right) P_{n}^{\prime}(\eta) \\
\psi^{i}=\sum_{n=1}^{\infty} b_{n}\left(r^{n+1}-r^{n+3}\right)\left(1-\eta^{2}\right) P_{n}^{\prime}(\eta)
\end{gathered}
$$

Here, and in the subsequent discussions, superscripts ' $O$ ' and ' $i$ ' refer to quantities outside and inside the active droplet respectively, $r$ is the radial coordinate nondimensionalised by droplet radius $R_{d}, \eta=\cos \theta$, and $P_{n}(\eta)$ is the Legendre polynomial of degree $n$ with the prime denoting its derivative; $n$ here physically represents the $n^{\text {th }}$ hydrodynamic mode. The non-dimensional radial and tangential flow velocity components around and inside the droplet are related to $\psi$ as $u_{r}=-\frac{1}{r^{2}} \frac{\partial \psi}{\partial \eta}$ and $u_{\theta}=-\frac{1}{r\left(1-\eta^{2}\right)^{1 / 2}} \frac{\partial \psi}{\partial r}$. The coefficients $a_{n}$ and $b_{n}$ in eqn. 3.4 and eqn. 3.5 are constrained by the following boundary conditions $[54,155]$ :

(i) tangential velocity $\left(u_{\theta}\right)$ condition at the droplet interface $(r=1)$ :

$$
u_{\theta}^{o}-u_{\theta}^{i}=m\left(\frac{2 \mu+3}{1+3 m}\right)\left(1-\eta^{2}\right)^{1 / 2}\left(\frac{\partial c}{\partial \eta}\right)_{r=1}
$$

(ii) tangential stress $\left(\tau_{r \theta}\right)$ condition at the droplet interface $(r=1)$ (Marangoni effect):

$$
\tau_{r \theta}^{o}-\tau_{r \theta}^{i}=-\frac{1}{\mu}\left(\frac{2 \mu+3}{1+3 m}\right)\left(1-\eta^{2}\right)^{1 / 2}\left(\frac{\partial c}{\partial \eta}\right)_{r=1}
$$

The coefficients on the right hand side of eqn. 3.6 and eqn. 3.7 essentially stem from the non-dimensionalization of the classical boundary conditions. Note that the flow velocity is non-dimensionalized using $V_{t}=\frac{q_{s}\left(\gamma_{c} R_{d}+3 \mu^{i} M\right)}{D\left(2 \mu^{\circ}+3 \mu^{i}\right)}$, which is a theoretical estimate for the terminal velocity of the active droplet considering the contributions of both 
the Marangoni and the diffusiophoretic effects [43, 54]. Furthermore, $\mu=\mu^{o} / \mu^{i}$ is the ratio of the swimming medium viscosity $\mu^{o}$ to the droplet viscosity $\mu^{i}$, and the non-dimensional parameter $m$ represents the relative strengths of diffusiophoretic to Marangoni effects [54]. Essentially, $m$ can be considered as a ratio of the diffusiophoretic velocity scale to the viscocapillary velocity scale representing the Marangoni effect. Accordingly, $m=\frac{\mu^{i} M}{\gamma_{c} R_{d}} \approx \frac{\zeta}{2 R_{d} \mu}$, where $M \approx \frac{k_{B} T}{2 \mu^{o}} \zeta^{2}$ is the diffusiophoretic mobility $[43,58], \gamma_{c} \approx k_{B} T \zeta$ is the leading order change in the interfacial surface tension $\gamma$ with surfactant concentration $c$ (alternatively, $\gamma_{c}=\frac{d \gamma}{d c}$ can be considered to be a measure of the change in $\gamma$ with $c$ assuming a linear variation) $[54,58]$, and $\zeta \sim 10 \mathrm{~nm}$ is the characteristic length scale over which the surfactants interact with the droplet in the interfacial region. For the active droplet system, $O(m) \sim 10^{-3}-10^{-2}$ for the entire range of experiments; hence, for the present physical problem the diffusiophoretic effect is much weaker as compared to the Marangoni effect. However, the former is considered in the analysis here for the sake of generality. In the definition of $V_{t}, q_{s}$ is an isotropic and constant interfacial surfactant consumption rate per unit area necessary for the droplet activity, and $D=\frac{k_{B} T}{6 \pi r_{s} \mu^{\circ}}$ is the diffusion coefficient for the surfactant monomer (length scale for surfactant monomer $r_{s} \sim 10^{-10} \mathrm{~m}$ ).

Eqn. 3.6 and eqn. 3.7 delineate the dependence of the swimming hydrodynamics on the distribution of the non-dimensional surfactant concentration $c$ in the vicinity of the droplet. Naturally, $c$ is governed by an advection-diffusion relation $[54,73,155]$ :

$$
P e\left[u_{r}^{o} \frac{\partial c}{\partial r}-\frac{u_{\theta}^{o}}{r}\left(1-\eta^{2}\right)^{1 / 2} \frac{\partial c}{\partial \eta}\right]=\frac{1}{r^{2}} \frac{\partial}{\partial r}\left(r^{2} \frac{\partial c}{\partial r}\right)+\frac{1}{r^{2}} \frac{\partial}{\partial \eta}\left(\left(1-\eta^{2}\right) \frac{\partial c}{\partial \eta}\right)
$$

The distribution of $c$ is subject to the following boundary conditions:

(i) isotropic and constant surfactant consumption at the droplet interface $(r=1)$

$$
\left(\frac{\partial c}{\partial r}\right)_{r=1}=1
$$

(ii) the bulk condition

$$
c(r \rightarrow \infty) \rightarrow c_{\infty}
$$

Note that eqn. 3.9 addresses the depletion of the interfacial surfactant monomers due to the creation of the filled micelles by considering the isotropic and constant interfacial surfactant adsorption rate per unit area of $q_{s}$, corresponding to a flux with unit of 
number per area per time (in dimensional form: $D \nabla c^{*} \cdot \hat{n}=q_{s}$; this gives a scale for the surfactant concentration as $\left.\sim \frac{q_{s} R_{d}}{D}\right) \quad[54,73]$. Pe in eqn. 3.8 is the system Péclet number- the details of which are discussed in the following sub-section. The above system of equations (eqn. 3.4-eqn. 3.10) can be solved for $\psi$ (therefore $u_{r}, u_{\theta}$ ), and $c$ using the singular perturbation technique for certain limiting cases $[54,73]$. The solvability condition clearly shows that the actuations of different hydrodynamic modes depend on certain threshold values of $P e$ (Fig. 4a in the main text) [54]. Furthermore, the asymptotic analysis also provides a physical understanding of the hydrodynamic and surfactant concentration fields corresponding to the different modes, specifically $n=1$ and $n=2$ (Fig. $4 \mathrm{~b}$ in the main text).

\section{The system Péclet number}

The important thing to understand now is the dependence of $P e$ on $\mu$. Classically, $P e$ can be written as $P e=\frac{V_{t} R_{d}}{D}$, where $V_{t}=\frac{q_{s}\left(\gamma_{c} R_{d}+3 \mu^{i} M\right)}{D\left(2 \mu^{o}+3 \mu^{i}\right)}$ is the theoretical estimate for the terminal velocity of the active droplet considering the contributions of both the Marangoni and diffusiophoretic effects, as mentioned in the preceding sub-section [43, 54]. Utilizing the aforementioned definition of $V_{t}$, and following some simple algebraic manipulations, $P e$ can be expressed in terms of system constants and the parameter $\mu$ as:

$$
\begin{aligned}
& P e=\frac{V_{t} R_{d}}{D}=\frac{q_{s}\left(\gamma_{c} R_{d}+3 \mu^{i} M\right)}{D\left(2 \mu^{o}+3 \mu^{i}\right)} \frac{R_{d}}{D} \\
& \Rightarrow P e=\frac{q_{s} M}{D m} \frac{(1+3 m)}{(2 \mu+3)} \frac{R_{d}}{D} \\
& \Rightarrow P e \approx \frac{18 \pi^{2}}{k_{B} T} q_{s} r_{s}^{2} \zeta R_{d}^{2} \mu^{i}\left[\mu\left(\frac{2 \mu+3 \zeta / R_{d}}{2 \mu+3}\right)\right]
\end{aligned}
$$

In the last step of eqn. 3.11, the approximate expressions for $M$ and $m$ (see subsection 3.9), and the definition of $D$ (see sub-section 3.9) are utilized to derive the final expression for Pe. Eqn. 3.11 expresses $P e$ as a monotonically increasing function of the viscosity ratio $\mu$ (markers in Fig. 4a in the main text). Note that $q_{s}$ is approximately estimated by relating the dissolution rate of the active droplet to the isotropic and constant surfactant consumption at the droplet interface [58]; the dissolution rate of the active droplet is dependent on the glycerol concentration (Fig. 3.8) which effectively 
makes $q_{s}$ dependent on $\mu_{o}$. We further note that the second term in the numerator within parenthesis $O\left(\frac{\zeta}{R_{d}}\right) \sim 10^{-4}$; this further substantiates the fact that the diffusiophoretic effect is much weaker compared to the Marangoni effect for the present system.

\section{Linear stability analysis about a motionless (isotropic) base state}

For the linear stability analysis (also see $[57,73]$ ), the time-dependent form of the advection-diffusion equation (eqn. 3.8) is used:

$$
P e\left[\frac{\partial c}{\partial t}-\frac{1}{r^{2}} \frac{\partial \psi^{o}}{\partial \eta} \frac{\partial c}{\partial r}+\frac{1}{r^{2}} \frac{\partial \psi^{o}}{\partial r} \frac{\partial c}{\partial \eta}\right]=\frac{1}{r^{2}} \frac{\partial}{\partial r}\left(r^{2} \frac{\partial c}{\partial r}\right)+\frac{1}{r^{2}} \frac{\partial}{\partial \eta}\left(\left(1-\eta^{2}\right) \frac{\partial c}{\partial \eta}\right)
$$

Next, the desired quantities are expressed in terms of the unsteady (instability) modes$\psi=e^{\lambda t} \sum_{n} \tilde{\psi}_{n}(r) P_{n}(\eta)$ and $c=-\frac{1}{r}+e^{\lambda t} \sum_{n} \tilde{c}_{n}(r) P_{n}(\eta)$, where $\lambda(>0)$ is the nondimensional growth rate for the instability modes. Using the aforementioned expressions for $\psi$ and $c$, and linearizing eqn. 3.12, the governing equations for the first two modes can be obtained as:

$$
\begin{aligned}
& \frac{d}{d r}\left(r^{2} \frac{d \tilde{c}_{1}}{d r}\right)-\left(2+\lambda_{s}^{2} r^{2}\right) \tilde{c}_{1}=2 \text { Pe } a_{1} \frac{1-r^{3}}{r^{3}} \\
& \frac{d}{d r}\left(r^{2} \frac{d \tilde{c}_{2}}{d r}\right)-\left(6+\lambda_{s}^{2} r^{2}\right) \tilde{c}_{2}=6 \text { Pe } a_{2} \frac{1-r^{2}}{r^{4}}
\end{aligned}
$$

where $\lambda_{s}=\sqrt{\lambda P e}$, and $a_{1}$ and $a_{2}$ are the coefficients of the first and second modes respectively of the outer stream function (as in eqn. 3.4). Eqn. 3.13 and eqn. 3.14 are solved to evaluate $\tilde{c}_{1}$ and $\tilde{c}_{2}$, respectively:

$$
\begin{aligned}
\tilde{c}_{1} & =\operatorname{Pe} a_{1}\left(\frac{2}{x^{2}}+\frac{\lambda_{s}^{3}}{2 x^{3}}\right)+\alpha_{1}\left(\frac{1+x}{2 x^{2}}\right) e^{-x}-\operatorname{Pe} a_{1} \frac{\lambda_{s}^{3}}{4 x^{2}}\left[\left(\frac{1+x}{2}\right)(\operatorname{Chi}(x)+\operatorname{Shi}(x)) e^{-x}\right. \\
& \left.-\left(\frac{1-x}{2}\right)(\operatorname{Chi}(x)-\operatorname{Shi}(x)) e^{x}\right]
\end{aligned}
$$




$$
\begin{aligned}
\tilde{c}_{2} & =P e \frac{a_{2}}{8}\left(\frac{8 \lambda_{s}^{4}}{x^{4}}+\frac{\lambda_{s}^{4}}{x^{2}}-\frac{6 \lambda_{s}^{2}}{x^{2}}\right)+\alpha_{2}\left(\frac{x^{2}+3 x+3}{2 x^{3}}\right) e^{-x} \\
& +P e a_{2} \frac{\lambda_{s}^{2}\left(6-\lambda_{s}^{2}\right)}{16 x^{3}}\left[\left(x^{2}+3 x+3\right)(\operatorname{Chi}(x)+\operatorname{Shi}(x)) e^{-x}\right. \\
& \left.-\left(x^{2}-3 x+3\right)(\operatorname{Chi}(x)-\operatorname{Shi}(x)) e^{x}\right]
\end{aligned}
$$

Here, $x=r \lambda_{s}$ is a rescaled spatial variable, $C h i(x)$ and $S h i(x)$ are the hyperbolic cosine integral and hyperbolic sine integral functions, and $\alpha_{1}$ and $\alpha_{2}$ are the constants of integration. Note that eqn. 3.15 and eqn. 3.16 are evaluated in a manner which satisfies the bulk condition for the surfactant distribution (eqn. 3.10) i.e. as $r \rightarrow \infty$, $c-c_{\infty} \rightarrow 0$. Furthermore, considering the expression for $c$, the interfacial surfactant consumption condition (eqn. 3.9) reduces to the form:

$$
\left(\frac{\partial c}{\partial r}\right)_{r=1}=1 \Rightarrow\left(\frac{d \tilde{c}_{1}}{d x}\right)_{x=\lambda_{s}}=0 ;\left(\frac{d \tilde{c}_{2}}{d x}\right)_{x=\lambda_{s}}=0
$$

Using eqn. 3.15 and eqn. 3.17, $\alpha_{1}$ can be evaluated as:

$$
\begin{aligned}
\alpha_{1} & =-\operatorname{Pe} a_{1} \frac{e^{\lambda_{s}}}{4\left(\lambda_{s}^{2}+2 \lambda_{s}+2\right)}\left[2\left(\lambda_{s}^{4}+6 \lambda_{s}^{2}+16\right)\right. \\
& +\lambda_{s}^{3}\left(\lambda_{s}^{2}-2 \lambda_{s}+2\right)\left(\operatorname{Chi}\left(\lambda_{s}\right)-\operatorname{Shi}\left(\lambda_{s}\right)\right) e^{\lambda_{s}} \\
& \left.-\lambda_{s}^{3}\left(\lambda_{s}^{2}+2 \lambda_{s}+2\right)\left(\operatorname{Chi}\left(\lambda_{s}\right)+\operatorname{Shi}\left(\lambda_{s}\right)\right) e^{-\lambda_{s}}\right]
\end{aligned}
$$

Similarly, using eqn. 3.16 and eqn. $3.17, \alpha_{2}$ can be evaluated as:

$$
\begin{aligned}
\alpha_{2} & =\text { Pe } a_{2} \frac{\lambda_{s}^{2} e^{\lambda_{s}}}{8\left(\lambda_{s}^{3}+4 \lambda_{s}^{2}+9 \lambda_{s}+9\right)}\left[-2 \lambda_{s}\left(5 \lambda_{s}^{2}+2\right)\right. \\
& +\left(\lambda_{s}^{2}-6\right)\left(\lambda_{s}^{3}-4 \lambda_{s}^{2}+9 \lambda_{s}-9\right)\left(\operatorname{Chi}\left(\lambda_{s}\right)-\operatorname{Shi}\left(\lambda_{s}\right)\right) e^{\lambda_{s}} \\
& \left.+\left(\lambda_{s}^{2}-6\right)\left(\lambda_{s}^{3}+4 \lambda_{s}^{2}+9 \lambda_{s}+9\right)\left(\operatorname{Chi}\left(\lambda_{s}\right)+\operatorname{Shi}\left(\lambda_{s}\right)\right) e^{-\lambda_{s}}\right]
\end{aligned}
$$

Eqn. 3.15 and eqn. 3.16, along with eqn. 3.18 and eqn. 3.19, give closed form expressions for $\tilde{c}_{1}$ and $\tilde{c}_{2}$.

Considering the hydrodynamic boundary conditions (eqn. 3.6 and eqn. 3.7), and using the orthogonality condition for Legendre polynomials, a set of two simple algebraic equations for the coefficients $a_{n}$, and $b_{n}$ for each of the first two modes can be written 
as:

(i) first mode $(n=1)$

$$
\begin{aligned}
3 a_{1}-2 b_{1} & =m\left(\frac{2 \mu+3}{1+3 m}\right) \tilde{c}_{1} \\
\mu a_{1}+b_{1} & =\frac{1}{6}\left(\frac{2 \mu+3}{1+3 m}\right) \tilde{c}_{1}
\end{aligned}
$$

(ii) second mode $(n=2)$

$$
\begin{aligned}
a_{2}-b_{2} & =\frac{m}{2}\left(\frac{2 \mu+3}{1+3 m}\right) \tilde{c}_{2} \\
\mu a_{2}+b_{2} & =\frac{1}{10}\left(\frac{2 \mu+3}{1+3 m}\right) \tilde{c}_{2}
\end{aligned}
$$

Note that $\tilde{c}_{n}$ in the above equations is explicitly dependent on $a_{n}$ (see eqn. 3.15, eqn. 3.18, and eqn. 3.16, eqn. 3.19). Considering the closed form expression for $\tilde{c_{1}}$ (eqn. 3.15 and eqn. 3.18), the solvability condition for eqn. 3.20 and eqn. 3.21 gives:

$$
\frac{-\left(\operatorname{Chi}\left(\lambda_{s}\right)-S h i\left(\lambda_{s}\right)\right) e^{\lambda_{s}} \lambda_{s}^{4}-\lambda_{s}^{3}+\lambda_{s}^{2}-2 \lambda_{s}+6}{12\left(\lambda_{s}^{2}+2 \lambda_{s}+2\right)}=\frac{1}{P e}
$$

Similarly, considering the closed form expression for $\tilde{c_{2}}$ (eqn. 3.16 and eqn. 3.19), the solvability condition for eqn. 3.22 and eqn. 3.23 gives:

$$
\begin{aligned}
& \frac{-\left(6-\lambda_{s}^{2}\right)\left(C h i\left(\lambda_{s}\right)-S h i\left(\lambda_{s}\right)\right) e^{\lambda_{s}} \lambda_{s}^{4}}{8\left(\lambda_{s}^{3}+4 \lambda_{s}^{2}+9 \lambda_{s}+9\right)}+\frac{\left(\lambda_{s}^{2}+2 \lambda_{s}+2\right)\left(\lambda_{s}^{3}-3 \lambda_{s}^{2}+6\right)}{8\left(\lambda_{s}^{3}+4 \lambda_{s}^{2}+9 \lambda_{s}+9\right)} \\
= & \frac{10}{P e} \frac{(1+\mu)(1+3 m)}{(2 \mu+3)(1+5 m)}
\end{aligned}
$$

eqn. 3.24 and eqn. 3.25 are solved numerically to evaluate the variations of the nondimensional growth rates $\left(\lambda=\frac{\lambda_{s}^{2}}{P e}\right)$ with $P e$ for the first and second instability modes respectively (Fig. 4c in the main text). Note that eqn. 3.24 is identical to that derived for the spontaneous motion of an autophoretic isotropic particle [57]. Furthermore, it is important to note here that the inverse of the time scale used for non-dimensionalizing the growth rate is $\frac{V_{t}}{R_{d}}$, which is consistent with the entire analysis.

\section{Supplementary figures}

Extending Fig. 3.3, Fig. 3.11 provides additional flow field snapshots to illustrate transient flow modes, with the chemical field kymographs plotted for a longer period of 60 seconds. Supporting Videos S6-8 respectively correspond to the kymographs in Fig. $3.11(\mathrm{a}-\mathrm{c})$. 
In Fig. 3.12, we have plotted the long-time tangential acceleration, speed and the reorientation angle for $P e=36$. This data set was used to identify the abrupt reorientation events. We identified these events based on a cutoff criterion for the reorientation between video frames $|\delta \theta|=0.2 \mathrm{rad}$ (Fig. 3.12, c \& d), aligned and overlaid the profiles of all events with the turning point $\left(\left|\delta \theta_{\max }\right|\right)$ set as $t=0$, and calculated the time-dependent average $(\langle\rangle$ represents ensemble averaging over all events).

In Fig. 3.13, we plotted the the long time acceleration signal for $P e=293$ to demonstrate signatures of bimodal swimming. Such events can be identified by intermittent strong fluctuations in the acceleration profile. The zoomed-in view further demonstrates the difference between stopping $(n=2)$ and swimming modes $(n=1)$. Constant transitions beween these modes result in the anomalous diffusive behaviour shown in Fig. 3.6 in the main text. 


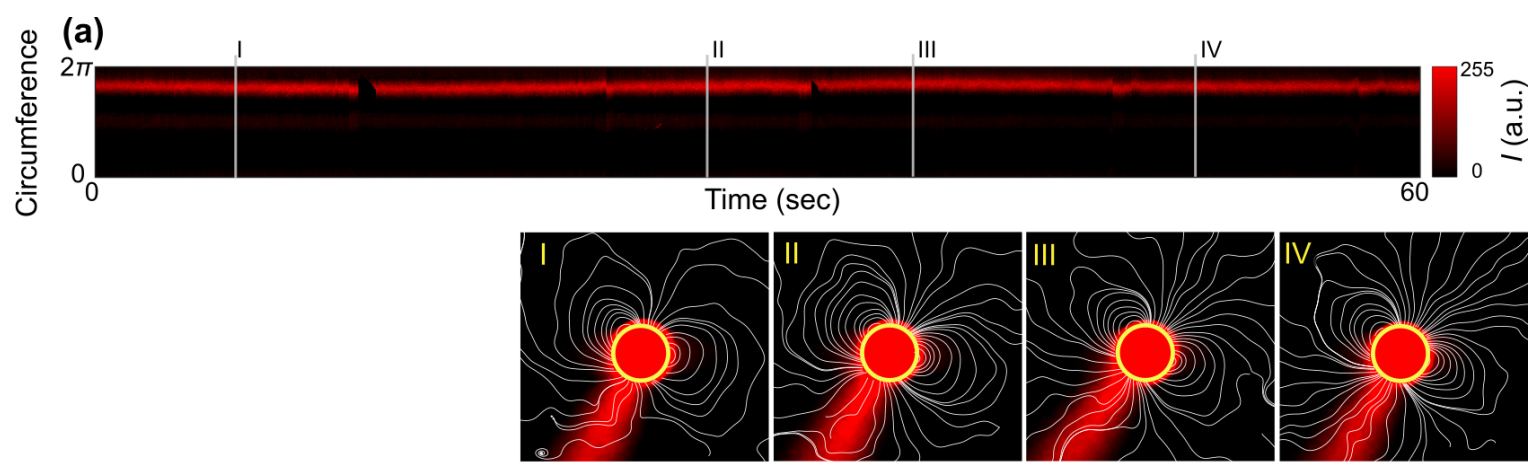

(b)
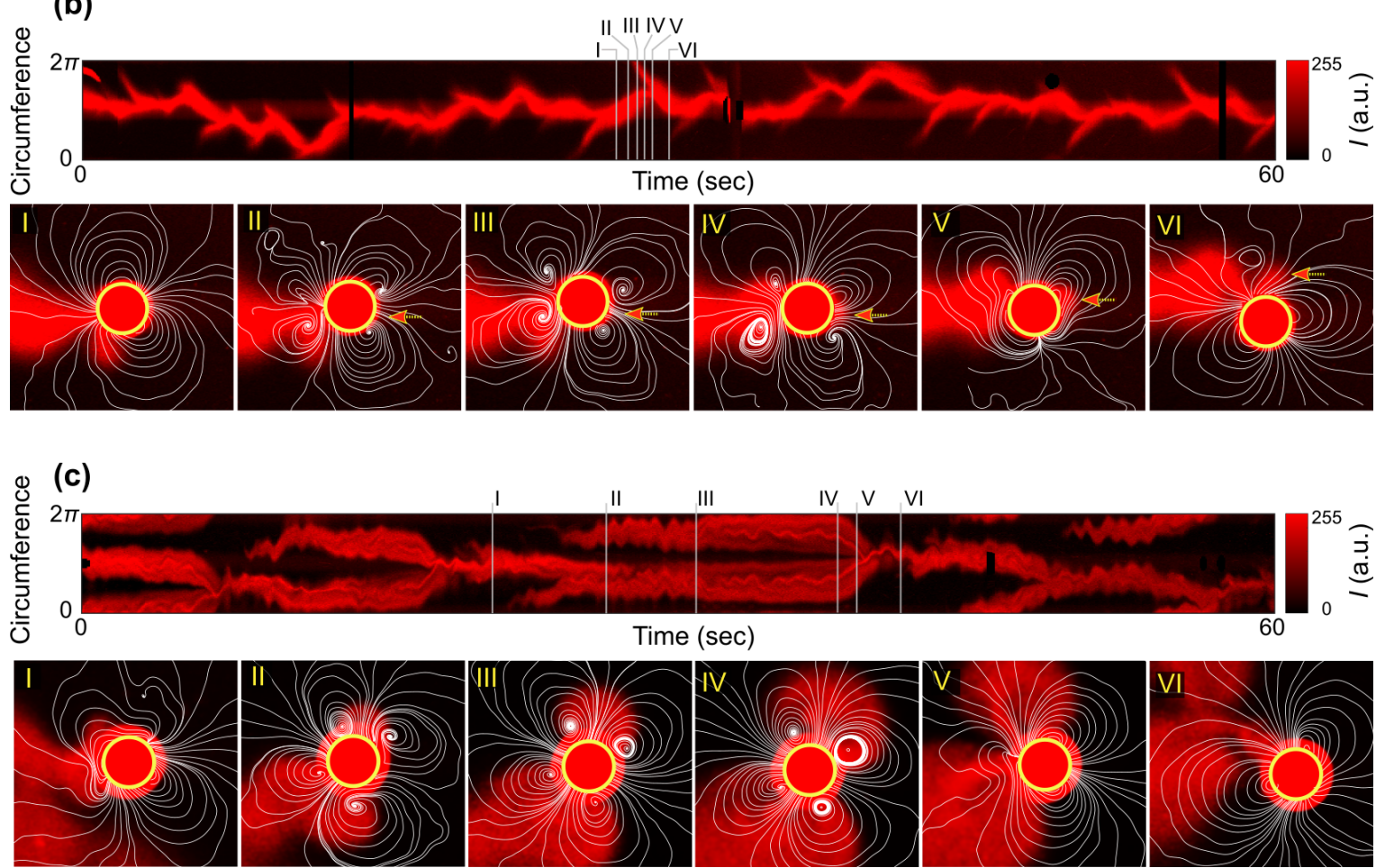

Figure 3.11 - Additional data for Fig. 3.3: Kymographs of the chemical field with selected instantaneous frames. Rows (a), (b) and (c) respectively correspond to $P e=4$, $P e=36$ and $P e=293$. In (b), the red arrow shows the location of the growing filled micelle blob. 
(a)

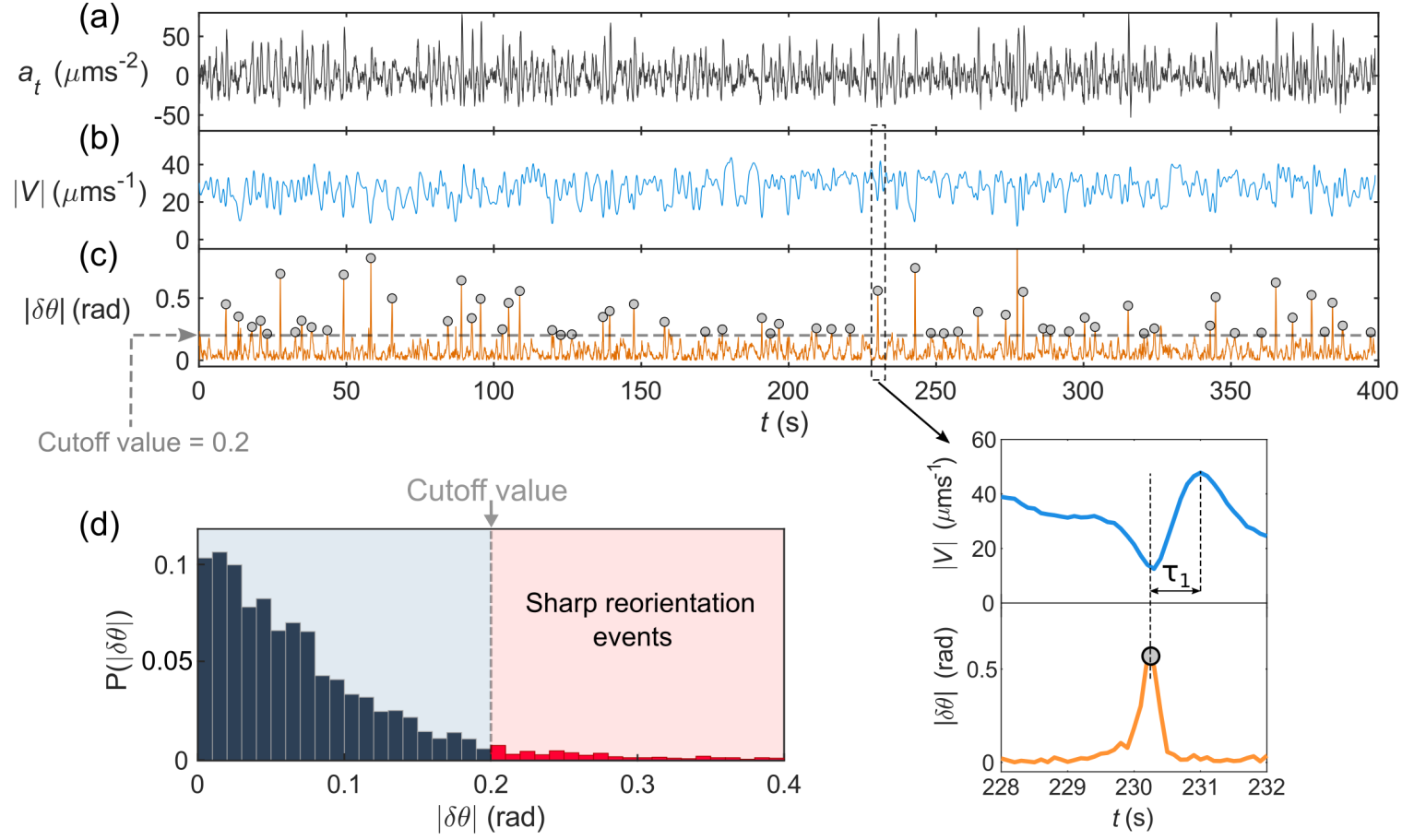

Figure 3.12 - Conditional averaging over reorientation events. (a), (b) and (c) are tangential acceleration, speed and the reorientation angle magnitude, respectively. In (c), the identified sharp reorientation events are shown by grey (o) symbols. The zoomed-in view is one example event that shows the general trend, a delay, $\tau_{1}$, between $|\delta \theta|$ and $|V|$. (d) The distribution of $|\delta \theta|$ and the cutoff value. The sharp turning events are coloured in red.

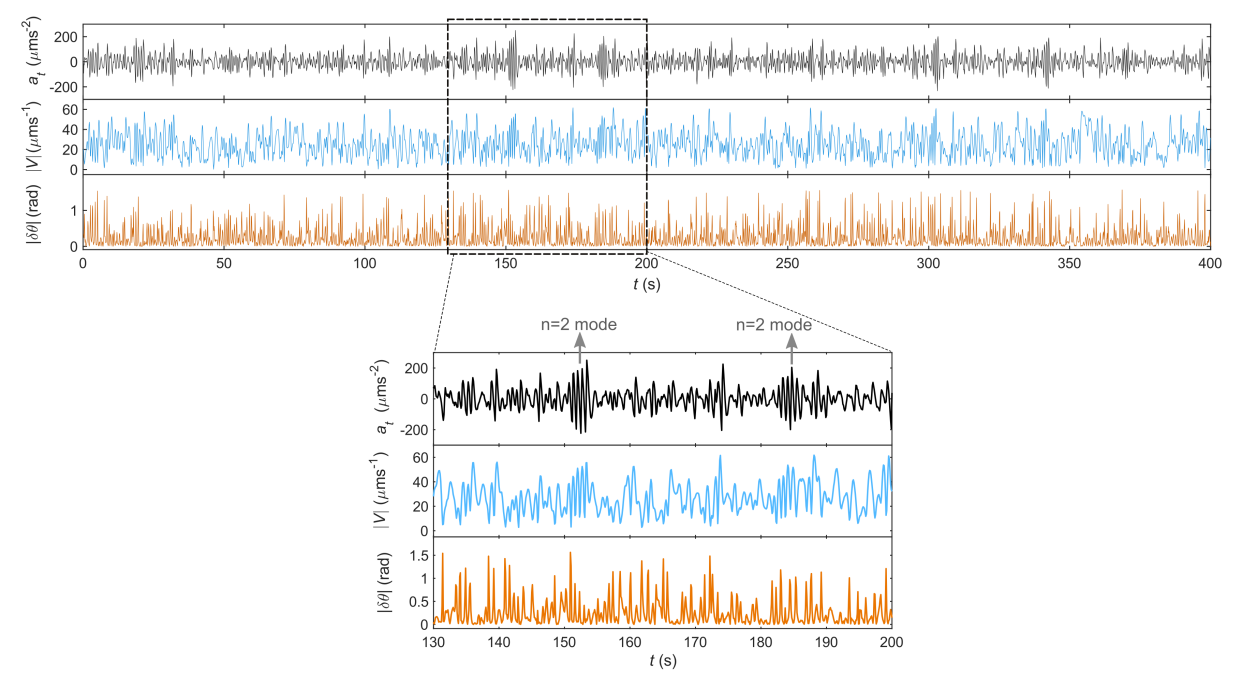

Figure 3.13 - Signatures of bimodal space exploration in the long-time tangential acceleration signal. The corresponding $P e$ is 293. 


\title{
Chapter 4
}

\section{Chemotactic droplet swimmers in complex geometries ${ }^{1}$}

\begin{abstract}
Chemotaxis $^{2}$ and auto-chemotaxis are key mechanisms in the dynamics of microorganisms, e.g. in the acquisition of nutrients and in the communication between individuals, influencing the collective behaviour. However, chemical signalling and the natural environment of biological swimmers are generally complex, making them hard to access analytically. We present a well-controlled, tunable artificial model to study chemotaxis and autochemotaxis in complex geometries, using microfluidic assays of self-propelling oil droplets in an aqueous surfactant solution $[13,116]$.Droplets propel via interfacial Marangoni stresses powered by micellar solubilisation. Moreover, filled micelles act as a chemical repellent by diffusive phoretic gradient forces. We have studied these chemotactic effects in a series of microfluidic geometries, as published in [89]: First, droplets are guided along the shortest path through a maze by surfactant diffusing into the maze from the exit. Second, we let auto-chemotactic droplet swimmers pass through bifurcating microfluidic channels and record anticorrelations between the branch choices of consecutive droplets. We present an analytical Langevin model matching the experimental data. In a previously unpublished experiment, pillar arrays

\footnotetext{
${ }^{1}$ This chapter is published in Jin, C., Hokmabad, B. V., Baldwin, K. A., and Maass, C. C. (2018). Chemotactic droplet swimmers in complex geometries. Journal of Physics: Condensed Matter, 30(5), 054003. Stop-and-go droplet swimmers. arXiv preprint arXiv:2005.12721. C. J. designed the research, performed experiments and data analyses and wrote the manuscript. B. V. H. performed experiments and data analyses and wrote the manuscript. K. A. B. performed experiments and wrote the manuscript. C. C. M. supervised the project and wrote the manuscript.

${ }^{2}$ Extended write-up of a talk given by C. Maass at Liquids 2017, presenting new data in the context of a review of our previous work on the subject.
} 
of variable sizes and shapes provide a convex wall interacting with the swimmer and, in the case of attachment, bending its trajectory and forcing it to revert to its own trail. We observe different behaviours based on the interplay of wall curvature and negative autochemotaxis, $i$. e., no attachment for highly curved interfaces, stable trapping at large pillars, and a narrow transition region where negative autochemotaxis makes the swimmers detach after a single orbit.

\subsection{Introduction}

One need only to look to nature to conclude that swimming techniques must be tailored to the swimmer's size. For a blue whale, for example, their enormity means their momentum dominates over viscous drag, and long drift times are the result. In contrast, for microscopic swimmers, such as algae or artificial microrobots, viscous forces will dominate, drift times become negligible, and the swimmer must sustain a continuous asymmetric flow pattern to achieve net motion, which in a Newtonian fluid is only possible via a time asymmetric swim-stroke, as shown by Purcell's infamous Scallop Theorem [171].

For microorganisms, propulsion is more than simply a means of random autonomous motion, it must also be responsive to the local environment, to reorient and propel the organism toward their various attractants. A few examples of this are chemical gradients, such as toward a food source (chemotaxis), flow gradients, such as towards a quiescent medium (rheotaxis), or light gradients, such as towards the Sun (phototaxis). Moreover, many organisms produce their own chemical gradients, to communicate their history with either neighbouring cells or their own future-selves (autochemotaxis). This latter example - a cell's ability to produce and respond to microbial chemical signals - is crucial for individual cell survival and colony formation.

Indeed, microbial life happens in complex bounded spaces - bacteria often live in soil, or are confined to biological tissues or fluid interfaces - which affects both the behaviour of the swimmers as well as the distribution of chemoattractants.

For the design of artificial microswimmers used as reactant vessels or chemical sensors, similar signalling pathways must also be considered if the swimmer is to reach a predetermined target, despite the many chemical, rheological and biological obstacles in its specific environment.

Taking lessons from biology for understanding and designing simple microscopic self-propulsion and signalling techniques is, however, complicated by the many other 
features of single-celled life: self-replication, irregular structure, metabolism, colony formation, mutation, and cell-death. To this end, various methods of artificial and biohybrid self-propulsion have been developed based on more rudimentary physical mechanisms. [41, 42, 58, 65, 172-177]

Active emulsions are a class of artificial swimmers that are particularly attractive as an ideal microswimmer as they are self-propelling in homogeneous fluids, are environment-sensitive, exhibit swimmer-to-swimmer chemical signalling, and are computationally simple due to their spherical symmetry. In this manuscript, based on a presentation given at Liquids 2017 in Ljubljana, we discuss our work on a specific type of active emulsion: liquid crystal droplets suspended in a cationic surfactant solution. These droplets display a rich variety of swimming behaviours, reminiscent of the stunning array of behaviours that micro-organisms exhibit. After a short review of current literature relating to the problem of chemoresponsive swimmers near interfaces in section 4.2 , sections 4.3 to 4.5 provide a review of the features of our specific system as previously published in $[13,79,89,116]$. In section 4.6 , we present an experiment on the interaction of droplets and circular pillars exhibiting a complex interplay of interfacial topology and phoretic effects.

\subsection{Interfacial interactions and chemotaxis in mi- croswimmers}

As argued above, the accurate physical modelling of biological systems in both theory and experiment is of high practical interest. Experimental and theoretical studies in this field evolve from the interplay of chemical gradients, interfaces and collective effects. The following review of current research should at least give a flavour of the complex task involved in bringing these concepts together. Doing the field of active swimmers fully justice would provide material for an entire monograph; we therefore restrict ourselves to a selection illustrating our specific problem and recommend to the reader extensive review articles like [178] and [179] and references therein.

A point much discussed in theoretical studies is that a swimmer is force and torque free by virtue of its self propulsion $[178,180]$. Setting up the required overdamped equations of motion is a delicate task. In the active Brownian particle model (ABP) [181], the forces and torques associated with self propulsion and the back action in the flow field are substituted with effective quantitites and balanced against external forces in 
the system, as in noise terms, steric effects, phoretic forces and taxes. This computationally efficient phenomenological approach has been successfully used in a number of studies to capture aspects of interfacial and collective interactions too complex for a full hydrodynamic treatment and is applicable to all classes of microswimmers ranging from bioswimmers over phoretic colloids to active emulsions. [182-185]

However, hydrodynamic flow fields can crucially determine the nature of interactions with obstacles, as e.g. observed in experimental studies on bioswimmer navigation at boundaries [186, 187]. Realistic studies need to test the validity of far field pusher/puller type and near field approximations, and quantitatively estimate the relative importance of hydrodynamic effects against steric or phoretic influences. Particularly in the case of interactions at curved interfaces, this force balance can determine the difference between scattering and entrapment [179, 188-193]. In our own experiments, we have observed large scale convective phase separation governed by hydrodynamic cutoff lengths and gravity [79].

A precise estimation of the force balance is specifically relevant if a phenomenological approach like an ABP model needs to be extended with respect to external influences like chemotaxis. Biological chemotaxis is usually not directly coupled to the chemoactive gradient. Small organisms like prokaryotes, due to their small size, have to sense gradients by time integration adapted to their typical propulsion strategies [194] run and tumble [195, 196], reverse and flick [197] or helical swimming [198]. Larger organisms able to probe gradients over their body length like amoeboids, leucocytes or dictyostelium [199, 200], can couple directly into spatial gradients, but in such cases comparative theoretical and artificial models have to accommodate intricate biomechanisms and related delay times, as has recently been demonstrated for a robotic system [201]. Delayed reactions are especially relevant for autochemotaxis [90, 202], where the gradients are produced by the active agents themselves and expected to change rapidly.

Chemotactic swimmers in complex environments have shown fascinating effects in a number of experiments on bioswimmers, [203-207] but these assays are quite often too complex for a full and quantitatively reliable analysis. Thus, there is a clear need for well controlled and tunable systems accessible to analytical modelling, and we believe active emulsions to be a promising candidate. 


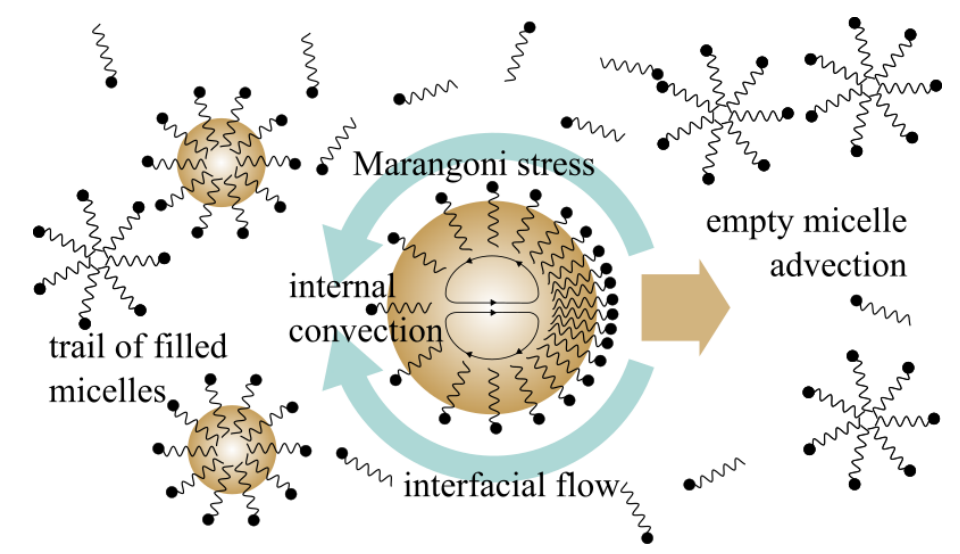

Figure 4.1 - Schematic of the surfactant coverage on the droplet interface, the distributions of empty and filled micelles and the flows involved in self propulsion.

\subsection{Active liquid crystal emulsions}

We begin with a review of the general properties of the system as previously published in $[13,116]$. We consider an emulsion of mesoscopic oil droplets in a micellar aqueous solution of an ionic surfactant. The oil disperses gradually into a micellar nanoemulsion, and the droplets self propel while dissolving, a motion that is caused by a self-sustaining gradient between the anterior and posterior poles of the droplet.

We model this interfacial instability as follows [13]: due to their charge, micelles are repelled from the interface, such that they are primarily filled in a boundary layer close to the interface by oil molecules diffusing in the water. This process requires the oil to be sparingly soluble in water. Micelles swell to accommodate the oil, thereby locally depleting the background of free surfactant to a concentration below the critical micelle concentration (CMC). Under these conditions, empty micelles must disintegrate and replenish both the $\mathrm{CMC}$ and the equilibrium interfacial coverage, but filled micelles are stabilized by the incorporation of oil molecules, and do not disintegrate. By this mechanism, the interfacial surfactant coverage is coupled to the local density of empty micelles outside the boundary layer, as it depends directly on the density of free surfactant in the aqueous phase at the interface. For a droplet at rest, all distributions are radially symmetric.

If a droplet is displaced by a random jolt, the initially spatially symmetric cloud of filled micelles around it will shift away from its front, and therefore the interfacial surfactant coverage at the anterior side of the droplet will increase, causing a gradient in interfacial tension towards the posterior (Fig. 4.1).

The resulting flow along the interface is coupled to both the aqueous and oil phases. 


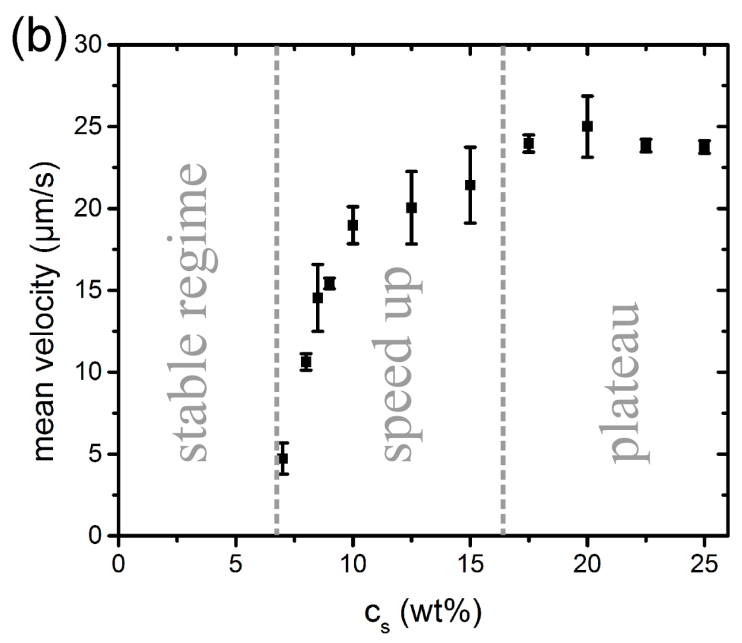

Figure 4.2 - Average speed of 5CB droplet swimmers in a Hele-Shaw cell, depending on the TTAB surfactant concentration in weight percentage. Figure adapted from [13], modified with permission of the Royal Society of Chemistry

In the aqueous phase, empty micelles are continually advected to the anterior interface, sustaining the surfactant coverage gradient, and thereby constant forward propulsion, while inside the droplet, there is a toroidal convective flow.

A qualitative analytical model of the advection-diffusion dynamics of micelles and molecules $[13,67]$ at the interface predicts a global threshold concentration of surfactant exceeding the $\mathrm{CMC}$, below which the interfacial coverage is stable against positional fluctuations, a range of increasing concentration during which the droplet will speed up, and a plateau in speed for high concentrations. We have confirmed this experimentally (Fig. 4.2).

Internal and external advective and convective flows affect two characteristic features of our self-propelling droplets. First, due to the direct coupling of the interfacial surfactant coverage to the local density of empty micelles, the droplets swim in the direction of positive surfactant gradients, i.e. exhibit chemotaxis. Since regions where droplets have previously traversed contain filled micelles (and therefore fewer empty micelles), droplets will avoid their own and other droplets' trails, i.e. exhibit negative autochemotaxis.

Second, droplets can be elastically anisotropic, if we use a nematic liquid crystalline oil phase. In this case, the internal toroidal flow will interact with the elastic field inside the droplet, causing broken symmetries and torques that lead the droplets on helical or looped trajectories [116]. We show a polarised micrograph of the defect structure in a droplet confined to a straight capillary and looped trajectories in a Hele-Shaw cell in 


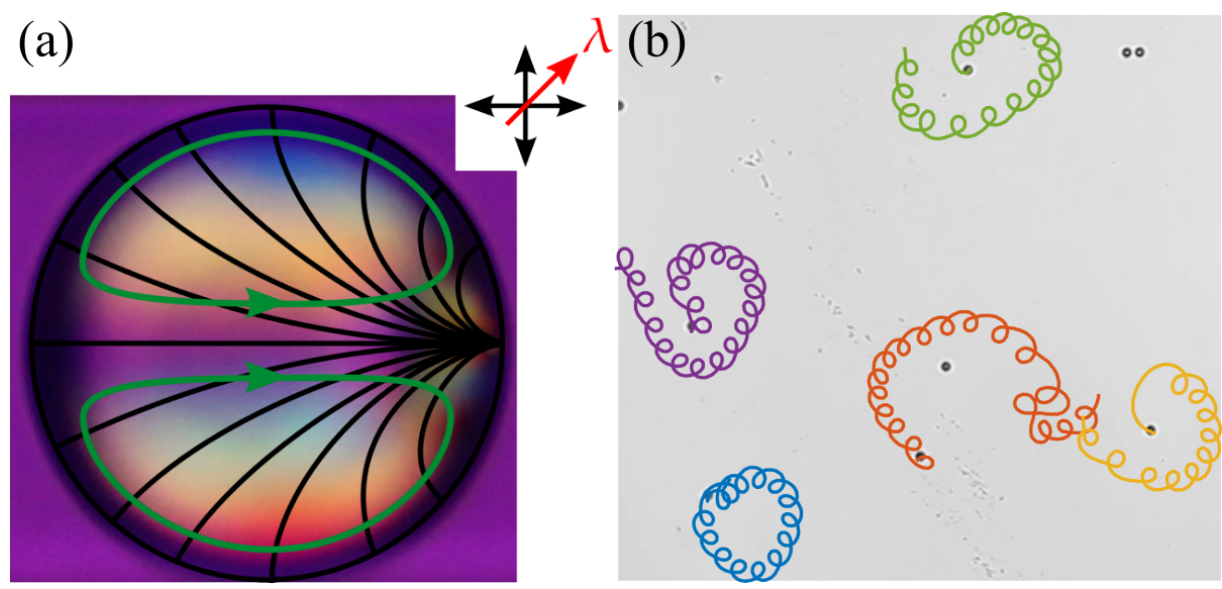

Figure 4.3 - (a) Polarised micrograph of a self propelling droplet with superimposed sketches of the nematic director field (black lines) and the internal convective flow (green lines). The central defect is pulled towards the anterior pole. (b) This type of elastic deformation causes helical swimming. Figure adapted from [116], with permission of the American Physical Society. Colour online.

Fig. 4.3. This behaviour can be reliably suppressed by using isotropic oils, in which case the swimming reverts to persistent random motion with a rather large persistence length around $10^{1}$ to $10^{2}$ droplet radii [116]. All data presented in the remainder of this paper are taken from analyses of isotropic swimmers.

\subsection{Methods}

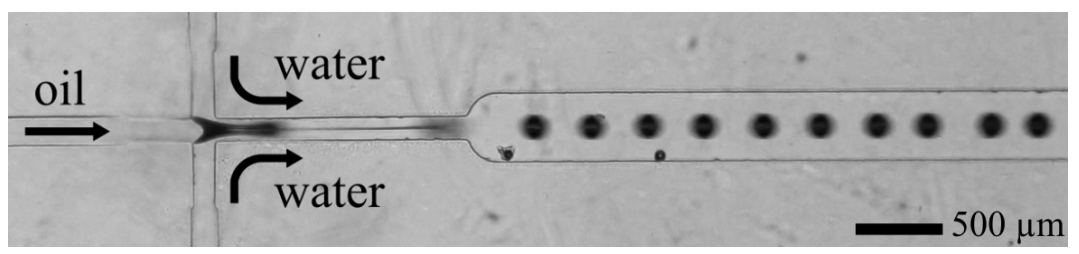

Figure 4.4-Monodisperse oil droplets (radius $50 \mu \mathrm{m}$ ) are mass produced in a microfluidic flow junction. The water phase contains surfactant at $c<c_{\mathrm{cmc}}$ to suppress coalescence.

All experiments presented in this paper have been conducted in quasi 2D microfluidic cells. Microfluidic devices are fabricated with standard soft lithography techniques [208], generating a Polydimethylsiloxane (PDMS) imprint of a SU8 photoresist stamp. The latter is cured in house using UV lithography and externally printed photomasks. The imprints are bonded to glass slides, connected to microfluidic pumps with Teflon tubing and mounted on microscope stages. These PDMS cells were used for both droplet production and experimental observations. 
Oil-in-water droplets are produced at high rates (10/s to $30 / \mathrm{s})$ and monodispersity with radii a ranging from $15 \mu \mathrm{m}$ to $50 \mu \mathrm{m}$ in cross-shaped flow junctions [209], as illustrated in Fig. 4.4. The oil phase consists of either: the nematogen 5CB, with a density of $1.04 \mathrm{~g} / \mathrm{cm}^{3}$ at room temperature and a nematic-isotropic phase transition at $35{ }^{\circ} \mathrm{C}$; an isotropic mixture of $5 \mathrm{CB}$ with $10 \%$ bromopentadecane, an insoluble oil of comparable density (5CB:BPD); or of the branched 5CB isomer CB15, commonly used as a chiral dopant, which in its pure form is isotropic at room temperature.

The continuous phase of our emulsions is an aqueous solution of the ionic surfactant TTAB, with a CMC of $c_{\mathrm{cmc}}=0.13$ wt.\%. For droplet production, we use a concentration of $c=0.1$ wt. $\%<c_{\mathrm{cmc}}$, sufficient to inhibit coalescence, but not high enough to support oil solubilisation or droplet swimming. Thus, stock emulsions are stable for months.

To initiate swimming, $\approx 0.3 \mu \mathrm{l}$ of stock solution is added to a $5 \mu \mathrm{l}$ to $10 \mu \mathrm{l}$ concentrated $c>30 c_{\mathrm{cmc}}$ surfactant solution, and the mixture is pipetted into the experimental container. Depending on their size and solubilisation rate, the droplets propel over a time scale between 20 min up to several hours, with a cruising range of several thousand droplet radii [13]. We observe them with bright field video microscopy and polarised video microscopy to image birefringent nematic liquid crystal phases. Numerical image analysis from video data provides reproducible dynamical and morphological data in statistically robust quantities.

\subsection{Chemotaxis and autochemotaxis}

The evolution of empty micelle distribution shapes the dynamics of our droplet swimmers, as demonstrated by the following three experiments in quasi $1 \mathrm{D}$ and $2 \mathrm{D}$ microfluidic geometries: droplet swimmers orienting towards a surfactant source, being guided through a maze by chemotaxis, and anticorrelated directional decisions guided by autochemotaxis. The latter two have been recently published in [89] and will be presented as a review.

As a consequence of the coupling between the density of empty micelles and the interfacial surfactant coverage described in sec. 4.3, swimmers will align their axis of motion with the gradient direction and swim towards higher surfactant concentrations, accelerating in the process. We first illustrate this in a simple experiment reminiscent of Adler's E. coli demonstrations, [196] where we placed a concentrated TTAB source at one end of a quasi 2D cell containing swimmers, and measured their speed and 

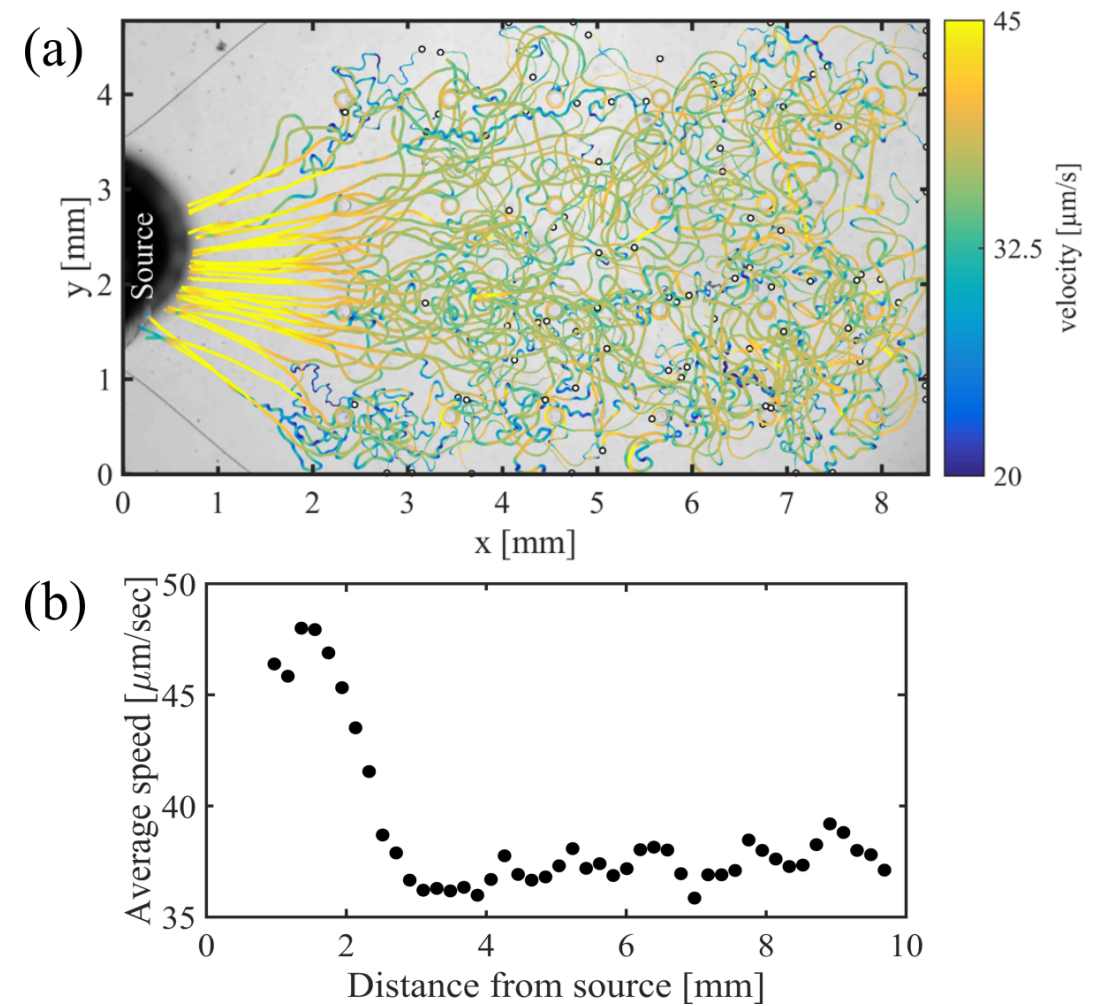

Figure 4.5 - TTAB gradient chemotaxis. (a) Trajectories of droplet swimmers after TTAB source is added to the left side of the microfluidic cell, colour coded by droplet speed. (b) Average speed as a function of distance for the data set in (a). Colour online.

distance from the surfactant source over time.

$50 \mu \mathrm{m}$ radius CB15 droplet swimmers were added to 5wt.\% TTAB solution, and the mixture was pipetted into one of our $10 \mathrm{~mm} \times 6 \mathrm{~mm}$ microfluidic cells, where the swimmers began persistent swimming immediately. After waiting for approximately 1 min to allow the transient flows initiated by pipetting to dissipate, a small quantity on the order of $10 \mathrm{mg}$ of pure TTAB powder was added to one of the two entry ports for this cell, which were then sealed to prevent evaporative flux. Over time, TTAB micelles diffused into the chamber from the pure powder, and the swimmers responded by reorienting their swimming towards the surfactant source, $i$. e. to the left in Fig. 4.5 (a), initially accelerating, and then reaching a steady maximum speed of $v \approx 45 \mu \mathrm{m} / \mathrm{s}$. This behaviour is evident in Fig. 4.5 from both the trajectories colour coded by speed (dark blue to light yellow, corresponding to slower to faster) in panel (a) and the average speed mapped to the distance from the source in panel (b). 

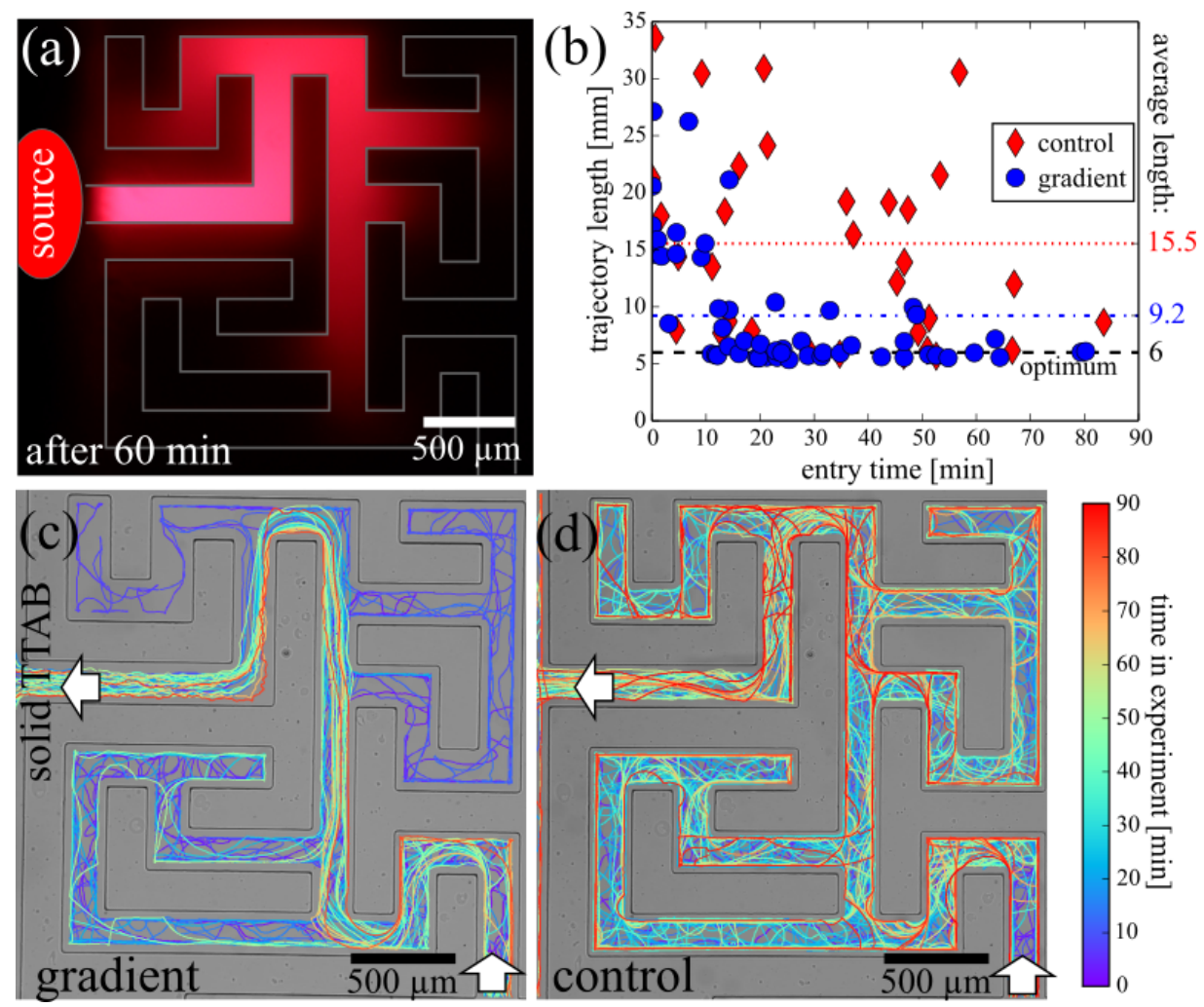

Figure 4.6 - (a) Fluorescently labelled surfactant spreading in a maze. (c) and (d) spreading surfactant guides swimmers along the shortest path (b) trajectory lengths from panels (c) and (d) vs. time of maze entry. Figure adapted from [89], with permission of the National Academy of Sciences (USA). Colour online.

\subsubsection{Maze solving}

We can exploit this tendency to prefer regions of higher surfactant density, and therefore reorientation in the direction of surfactant gradients, for guidance in complex geometries. Fig. 4.6, from [89], shows this for droplets swimmers in a microfluidic maze structure connecting an entry and an exit reservoir. The maze was prefilled with a surfactant solution enabling self propulsion (5wt.\% TTAB); we then simultaneously added droplet stock solution to the entry and TTAB powder to the exit reservoir, cut off external flow and observed how droplet swimmer trajectories progressively aligned along the shortest path through the maze while the added surfactant spread through the structure.

Accompanying time lapse observations of the spread of surfactant dyed with Nile Red inside the same maze without swimmers (panel (a)) showed that the additional surfactant develops a gradient along the shortest path, guiding the swimmers.

Panel (b) shows the length of individual trajectories inside the maze versus the time at which the swimmer passes the entry point for a maze with ("gradient") and without 

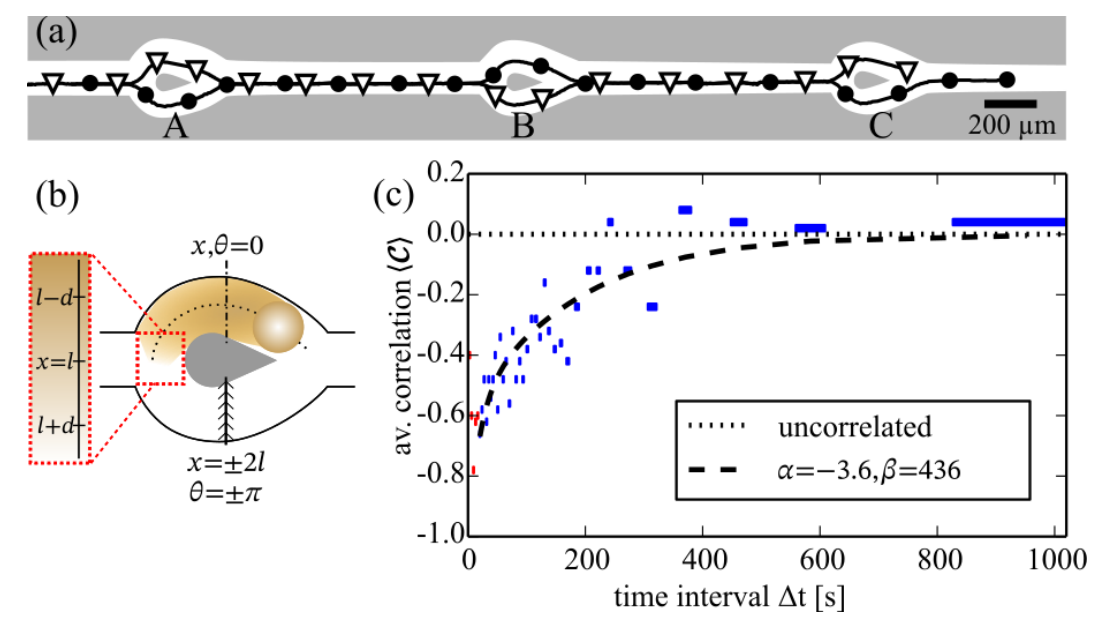

Figure 4.7 - (a) a pair of swimmers choosing alternating branches around pillars in a channel. (b) Pillar schematic with length definitions for eqs. 4.1 to 4.3. (c) anticorrelation data from experiments, with a fit to eqn. 4.3. Figure adapted from [89], with permission of the National Academy of Sciences (USA)

("control") added solid surfactant. For the gradient experiment, the trajectories revert to the shortest path when the surfactant has spread through the maze, while no such effect is present in the control experiment. Panels (c) and (d), using the same data, illustrate this by mapping the trajectories, colour coded by the time in the experiment, onto a micrograph of the maze structure.

\subsubsection{Diffusion controlled autochemotaxis}

To study autochemotactic effects, we designed an experiment where a series of swimmers passes through a channel containing a series of tear-drop shaped bifurcations, where the flow splits at the rounded side, and recombines at the tip.

We observed that consecutive swimmers prefer alternating branches of the same bifurcation and that this anticorrelation decays with time $\Delta t$ between passages. Fig. 4.7 (a) shows two swimmer trajectories plotted over a channel with 3 bifurcations. In this example, the swimmers passed in very close sequence, $\Delta t<10 \mathrm{~s}$, and all branch choices are anticorrelated.

We now give a summary of a simple model of the anticorrelation decay over time, based on micellar diffusion. A full derivation can be found in [89].

We consider the passage of two consecutive swimmers through a bifurcation, where the first swimmer randomly chooses a branch path (in this case the upper path), and our analysis begins at time $\Delta t=0$, as this swimmer leaves the junction. 
We assume that the fluid in the lower branch is unaffected by the swimmer's passage prior to $\Delta t=0$, whereas the upper branch is filled with an initially constant concentration of swollen micelles, which then diffuse around the pillar. Thus, the second swimmer will encounter a gradient in the concentration of filled micelles $\partial_{x} c(\Delta t)$, corresponding to an autochemotactic drift force downwards, with a coupling constant $\kappa$. Adding a Gaussian noise term $\Gamma$ to model a random branch choice of a swimmer with diffusion coefficient $D_{s}$, we set up the Langevin equation of motion for the second swimmer entering the junction at $x=l$ (see Fig. 4.7 for length definitions):

$$
\left.\frac{d x}{d \tau}\right|_{x=l}=\kappa \partial_{x} c+\sqrt{2 D_{s}} \Gamma(\tau) .
$$

Calculating the probability at the two absorbing boundaries at $l \pm d$ yields an expression for the average correlation depending on the time evolution of the surfactant gradient:

$$
\begin{aligned}
\langle\mathcal{C}\rangle & =\tanh (\xi(\Delta t)) \\
\xi & =-\frac{\kappa \cdot d}{2 D_{s}} \partial_{x} c(\Delta t) \\
& =-\frac{\alpha}{\sqrt{t}}\left(2 \exp \frac{-\beta}{t}-1-2 \exp \frac{-4 \beta}{t}+\exp \frac{-9 \beta}{t}\right)
\end{aligned}
$$

with two parameters, a prefactor $\alpha$ and a time constant $\beta$ :

$$
\alpha=\gamma \frac{\kappa}{D_{s} \sqrt{D_{m}}}, \beta=\frac{l^{2}}{D_{m}} .
$$

Here, $\gamma$ is a prefactor combining experimental parameters (see eq. 7 in [89]). We can interpret $\alpha$ as a measure of chemotactic strength versus Brownian noise, while $\beta$ corresponds to the decay time based on micellar diffusion. We have plotted the average correlation for $\approx 4000$ passage pairs and a fit derived from eq. 4.3 in fig. 4.7 (c). There is no absolute value for $\alpha$ as the diffusion constant of a confined active droplet is ill defined; however, our measured value of $\beta$ is consistent with the known values for the micellar diffusion constant $D_{m}$ and the size of the bifurcation $l$.

This model provides reasonable evidence that the autochemotactic force is linearly coupled to the gradient in filled micelles, which can be modelled reliably by micellar diffusion. 


\subsection{Chemotactic effects at pillars}
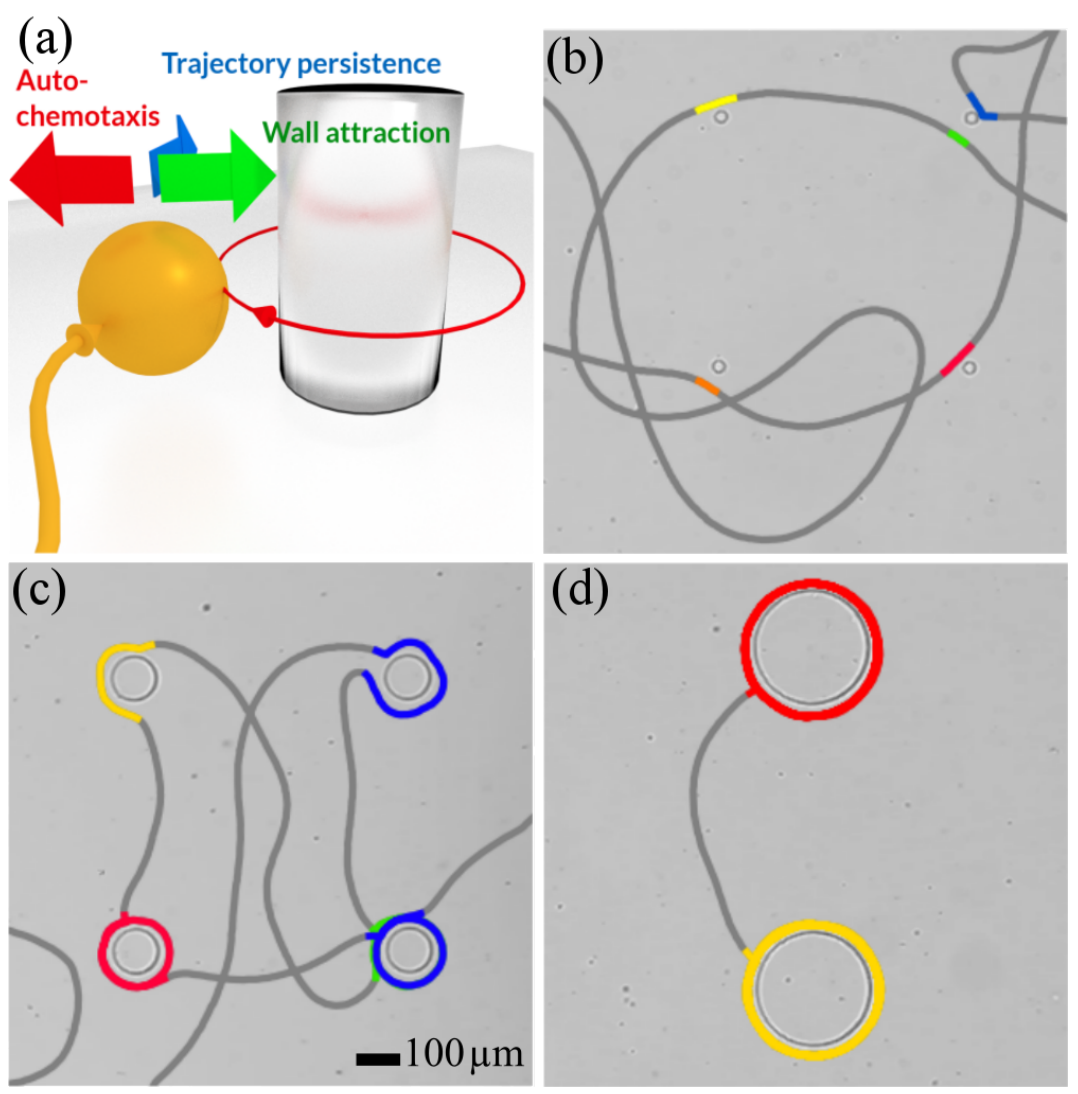

Figure 4.8 - (a) Schematic of attractive and repulsive forces involved in the swimmerpillar interaction. (b)-(d) Example trajectories of swimmers at $30 \mu \mathrm{m}, 100 \mu \mathrm{m}$ and $250 \mu \mathrm{m}$ pillars, with sections identified as attached by numerical filtering marked in colour. We observe a transition from bouncing to trapping with an intermediate regime of one-orbit trapping. Movies for panels (b)-(d) are included in the supplementary information.

We demonstrate in a simple model system how chemotactic signalling can shape the interaction of swimmers and interfaces to the point of switching from attraction to repulsion, using quasi-2D PDMS cells containing arrays of pillars of variable sizes. As outlined in sec. 4.2, previous experimental studies and theoretical models of biological and artificial swimmers at pillars have shown a transition from scattering to trapping for increasing pillar sizes [190, 192, 210]. However, in our swimmers we have observed an additional intermediate regime where droplets leave after circling the pillar only once due to chemotactic repulsion (Fig. 4.8). Attachment statistics were derived from the video tracking of droplets in regular microfluidic pillar arrays (Fig. 4.9).

We have observed that droplet swimmers are generally attracted to interfaces; the hydrodynamic and phoretic contributions to this attraction are yet to be determined. 


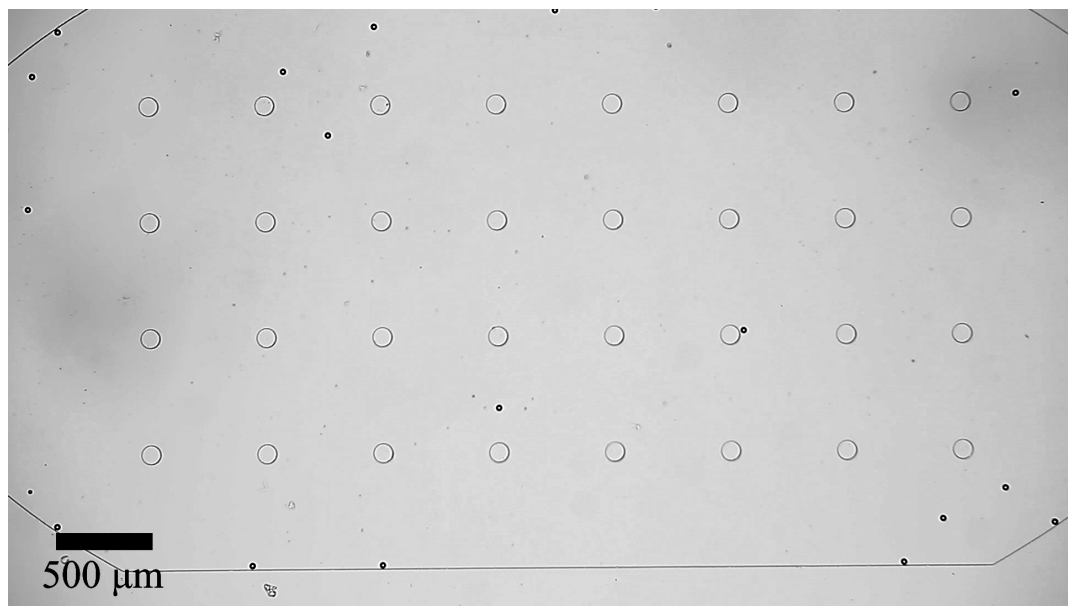

Figure 4.9 - Experimental screenshot of a PDMS cell with a $100 \mu \mathrm{m}$ pillar array. The contrast of this image has been modified for clarity.

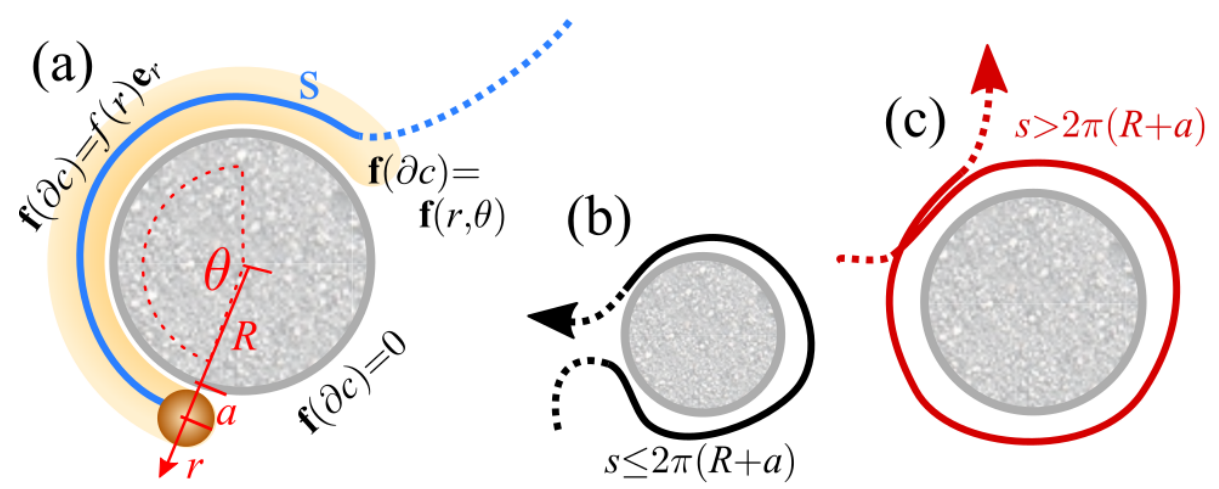

Figure 4.10 - (a) Schematic of the filled micelle gradients leading to a radial chemotactic force at a pillar (b) a non-crossing trajectory (c) a self crossing trajectory

In continuous quasi $2 \mathrm{D}$ and $3 \mathrm{D}$ media, they move in a very persistent manner with little rotational fluctuation. Attachment to a pillar of radius $R$ much smaller than the persistence length of the swimmer would require a sudden reorientation with very little interfacial area to mediate attraction. Thus, the swimmer is likely to scatter off the pillar, or to detach very easily, resulting in a distribution $P(s)$ of attachment lengths $s$ tailing off well below a pillar orbit, $s=2 \pi(R+a)$, for a swimmer of radius $a$. For pillars larger than the persistence length, little reorientation is required and the wall attraction leads to near indefinite trapping, $s \rightarrow \infty$. This behaviour is comparable to the aforementioned studies.

However, for intermediate pillar sizes, we need to consider that a swimmer circling a pillar will after one orbit encounter its own trail of filled micelles, which corresponds to an additional chemotactic gradient force $\boldsymbol{f}(\partial c)$ pointing away from the pillar. We have sketched the micelle distribution around a pillar in Fig. 4.10 (a), using polar 
coordinates $(r, \theta)$ with the origin at the pillar centre. During the first orbit of the pillar, $r \approx(R+a), \theta<2 \pi$, there are no filled micelles in the path of the swimmer and $\boldsymbol{f}(\partial c)$ is zero. When the swimmer encounters the rear end of its own trail, the filled micelle density will increase with $\theta: \boldsymbol{f}(\partial c)=\boldsymbol{f}(r, \theta), \partial_{\theta}|\boldsymbol{f}|>0$. After that, for a swimmer traveling on its trail at constant speed, the force will be radial and constant in $\theta, \boldsymbol{f}(\partial c)=f(r) \boldsymbol{e}_{r}$, during each orbit, $2 \pi n<\theta<2 \pi(n+1)$. Since filled micelles diffuse away from the pillar over time, $f(r)$ decreases with the increasing orbiting period of the swimmer, leading to weaker repulsion for larger pillars or slower swimmers.

Combining the effects of persistence, wall attraction and chemotactic repulsion, there is an intermediate pillar size where the first two are of the same order, but the chemotactic force will shift this balance towards repulsion. This shift can be so pronounced that the swimmer is already repelled when it encounters the increase in filled micelles at the rear of its own trail, resulting in a non-crossing pillar orbit (Fig. $4.10 \mathrm{~b}$ ). If the chemotactic repulsion is sufficiently small to enable the swimmer to travel on its own trail (Fig. $4.10 \mathrm{c}$ ), the swimmer will detach stochastically, depending on the strength of $f(r)$, such that the peak of $P(s)$ at one orbit develops a tail that increases for larger pillar sizes and respectively weakening $f(r)$. Eventually, in the limit of very large pillars, $P(s)$ will show an increase for very long trapping times.

We have observed a transition from scattering to one orbit trapping to indefinite trapping in experiments, using 5CB:BPD droplet swimmers of radius $a=15 \mu \mathrm{m}$ in regular pillar arrays with radii $R$ varying from $15 \mu \mathrm{m}$ to $125 \mu \mathrm{m}$.

We filled pillar arrays with a 7.5wt.\% TTAB solution containing small quantities of swimmers $(n<5 / \mu l)$ and recorded the swimmer dynamics on a bright field microscope under $4 \mathrm{x}$ magnification. For one pillar size, we typically have data from more than 20 experiments of $5 \mathrm{~min}$ to $10 \mathrm{~min}$ with 5 to 20 swimmers and 1 to 200 individual pillar interactions per experiment. We numerically extracted the swimmer trajectories, identified all trajectory segments corresponding to pillar attachment and binned them by segment length $s$, normalised to the pillar circumference, to collect statistics for the attachment length distribution $P(s)$.

The experimental screenshot in Fig. 4.9 shows a typical cell geometry with pillar radius $R=50 \mu \mathrm{m}$. Fig. 4.8 illustrates the transition with example trajectories taken from experiments using swimmers of radius $a=15 \mu \mathrm{m}$, with the numerically extracted interaction segments $s$ marked in colour. We see scattering off $15 \mu \mathrm{m}$ pillars, both self crossing and non-crossing one orbit trapping at $50 \mu \mathrm{m}$ pillars and indefinite trapping at $125 \mu \mathrm{m}$ pillars. 

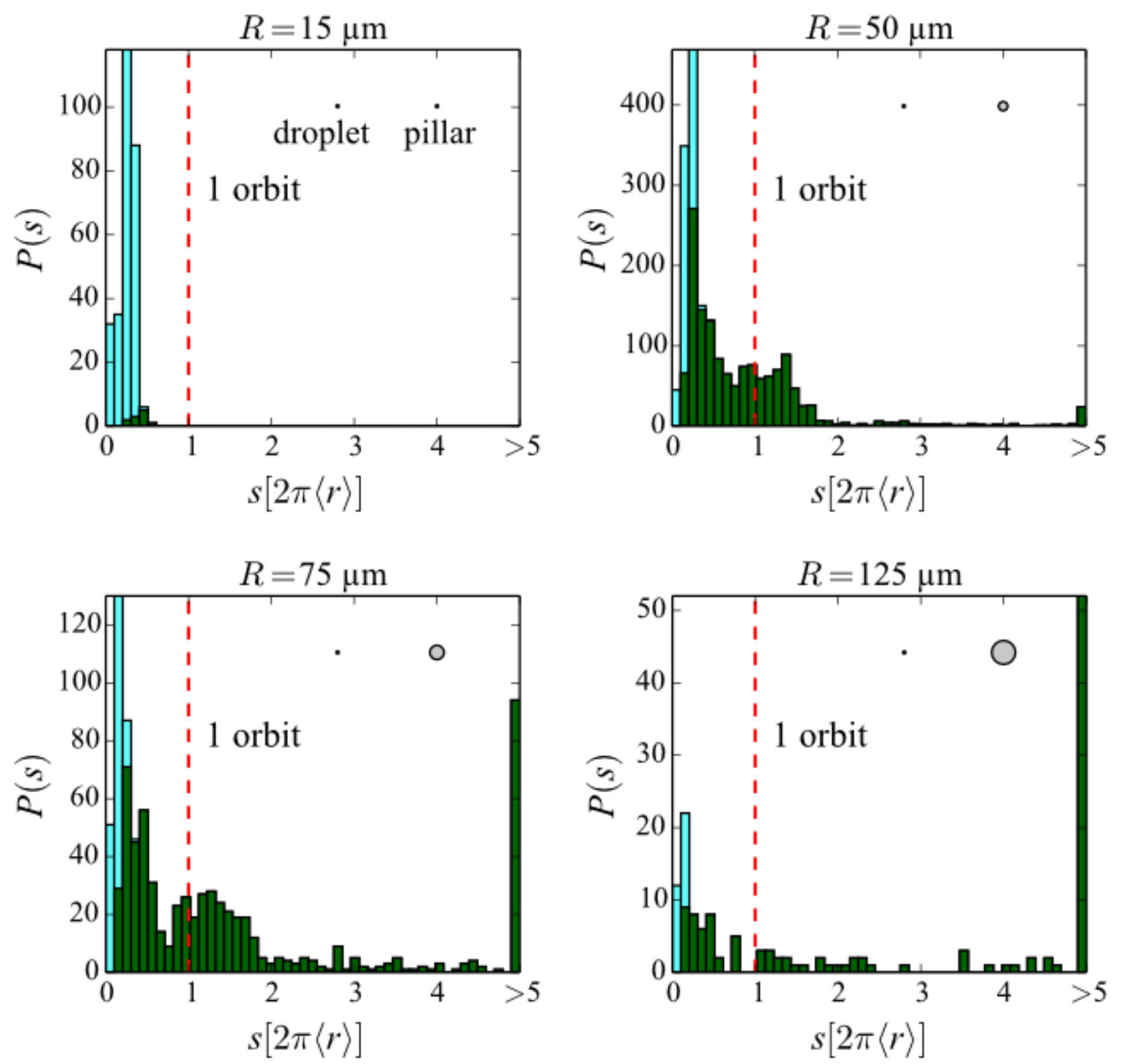

Figure $4.11-a=15 \mu \mathrm{m}$ swimmers: Scattering for $R=15 \mu \mathrm{m}$ pillars, onset of one orbit trapping at $R=50 \mu \mathrm{m}$ pillars and transition to indefinite trapping for $R=125 \mu \mathrm{m}$ pillars. We plot data from scattering interactions in light cyan and data from attachment interaction in dark green. 

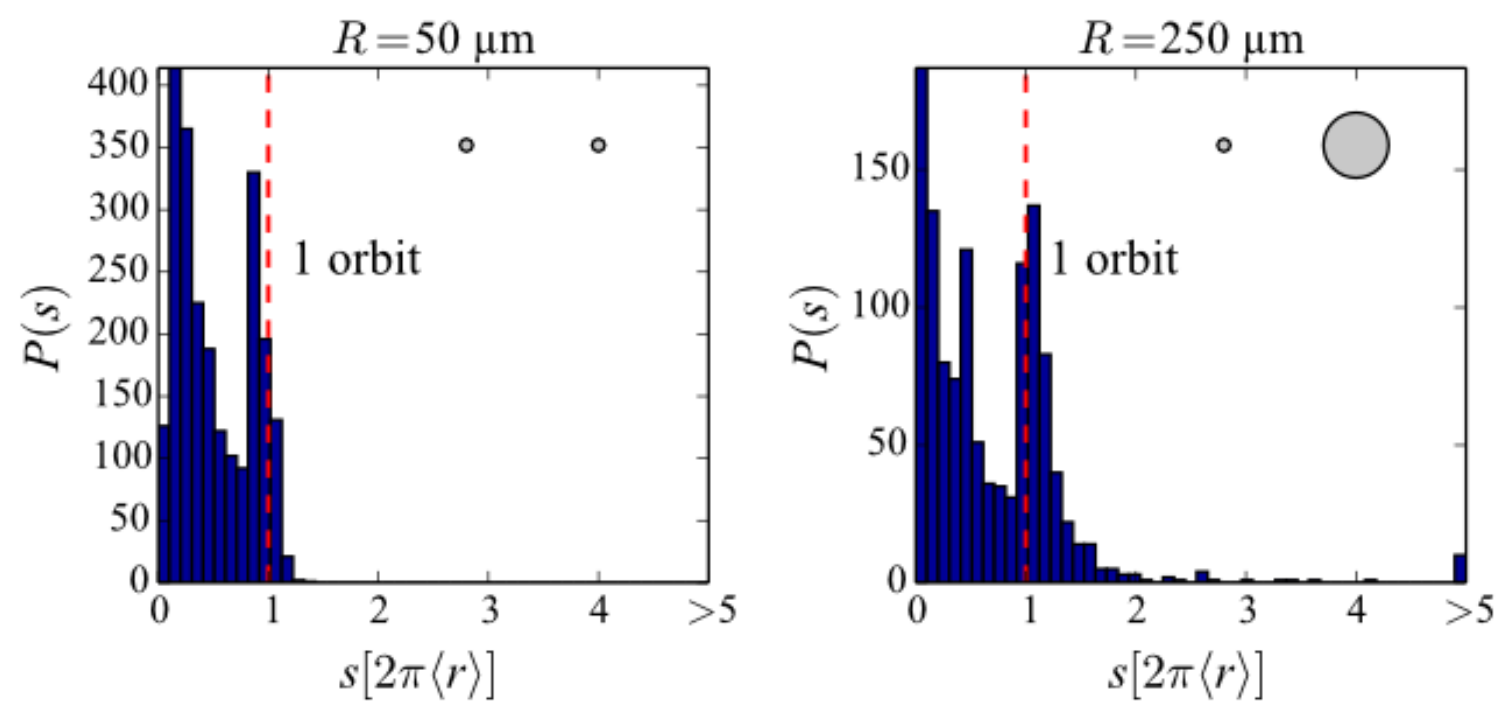

Figure $4.12-a=50 \mu \mathrm{m}$ swimmers: Onset of one orbit trapping at $R=50 \mu \mathrm{m}$ pillars and transition to indefinite trapping for $R=250 \mu \mathrm{m}$ pillars. This range is studied in more detail in Fig. 4.13 to resolve the transition from self crossing to non-crossing trajectories.

We begin with a statistical analysis of the full range of pillar interactions from scattering to trapping using swimmers of radius $a=15 \mu \mathrm{m}$. We consider a swimmer to be attached if its direction of motion calculated from the trajectory's tangent is changed by pillar attraction, in all other cases we consider it to have scattered off the pillar. Histograms of $P(s)$ vs. $s$ for 4 pillar radii, $15 \mu \mathrm{m}, 50 \mu \mathrm{m}, 75 \mu \mathrm{m}$ and $125 \mu \mathrm{m}$, are plotted in Fig. 4.11, showing a pronounced peak corresponding to one orbit trapping around pillars of $R=50 \mu \mathrm{m}$ between regimes of scattering for small pillars and trapping for large pillars.

Since we are able to reliably identify self intersecting trajectories, we have studied the transition from non-crossing to self-crossing pillar interactions in more detail for $a=50 \mu \mathrm{m}$ swimmers at pillars in a radius range from $50 \mu \mathrm{m}$ to $250 \mu \mathrm{m}$ between the onset of one orbit trapping and the transition to indefinite trapping. Here, the one orbit peak is more pronounced, as scattering is weak for larger swimmers. We note in the comparison of $a=15 \mu \mathrm{m}$ and $50 \mu \mathrm{m}$ data that $P(s)$ cannot be rescaled easily to the relation of swimmer and pillar radii, as the swimmer speed, persistence length and solubilisation rates depend nonlinearly on the swimmer size as well.

The histograms in Fig. 4.12 represent the approximate boundaries of the regime where one orbit trapping is dominant, i.e. pillar radii of $50 \mu \mathrm{m}$ and $250 \mu \mathrm{m}$. This data range is resolved in more detail in Fig. 4.13, with pillar radii of $50 \mu \mathrm{m}, 75 \mu \mathrm{m}, 100 \mu \mathrm{m}$ and 

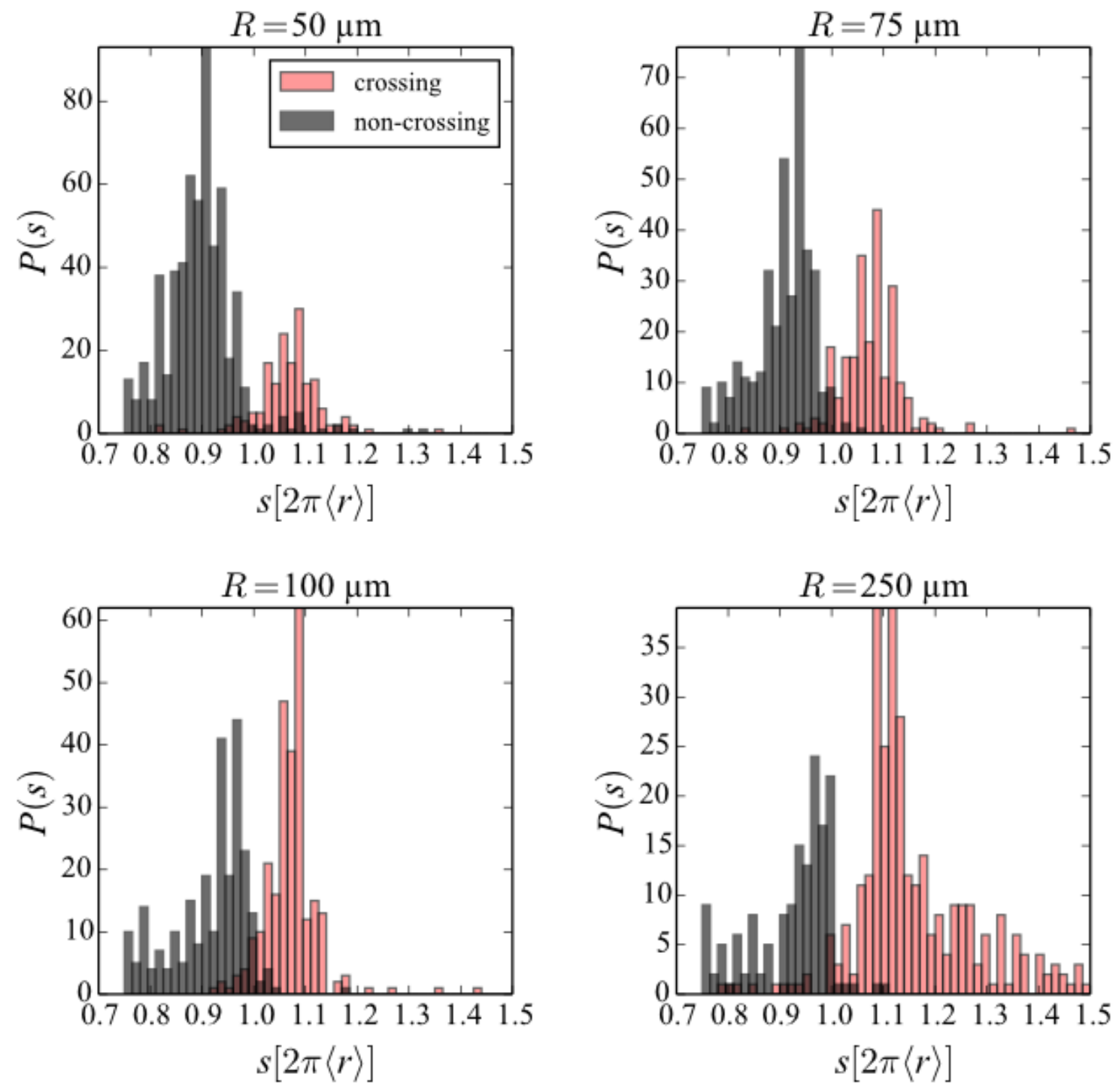

Figure 4.13 $-a=50 \mu \mathrm{m}$ swimmers: For increasing pillar radii, attachment events in the one orbit range shift from non-crossing (dark grey) to self-crossing (light red) trajectories. 
$250 \mu \mathrm{m}$ and a length range focusing on the one orbit peak, $0.75(2 \pi\langle r\rangle)<s<1.5(2 \pi\langle r\rangle)$. We furthermore separate the recorded events into self crossing (red) and non-crossing trajectories (grey) and observe a clear shift towards the self crossing regime for increasing pillar sizes, as well as the emergence of a tail towards more stable attachment. In future work, we will fit these statistics with an analytical stochastic model analogous to the one outlined in Sec. 4.5.2.

\subsection{Conclusion}

In a straightforward demonstration experiment combining the effects of complex boundaries and chemotactic forces on droplet swimmers at complex interfaces, we have reproduced established phenomena of pillar scattering and trapping and observed a new one orbit trapping regime directly caused by chemotactic effects. We note that this effect is observable due to the slow diffusion of the chemorepellent. We have furthermore sketched an avenue towards quantitatively evaluating our data in the context of an active particle model.

One of the advantages of the active emulsion model is the wide range of controllable parameters and inspection methods. While we have not yet included hydrodynamic effects in the present study, it is both feasible experimentally via micro-PIV and interferometry to map flow fields and lubrication effects. Moreover, by virtue of the high symmetry of the system, active droplets are suitable for very detailed numerical and analytical analysis. Focussing all these approaches on the same experimental parameters should offer important insight on the cooperation of hydrodynamic, phoretic and steric effects in active media.

\section{Acknowledgments}

We thank Stephan Herminghaus, Christian Bahr, Marco Mazza, Fabian Schwarzendahl and Rebekka Breier for valuable discussions. This work was supported by the DFG's SPP 1726 "Microswimmers" and the MPG's MaxSynBio initiative. We also thank the organisers of Liquids 2017. 



\title{
Chapter 5
}

\section{Quantitative characterization of chemorepulsive alignment-induced interactions in active emulsions ${ }^{1}$}

\begin{abstract}
The constituent elements of active matter in nature often communicate with their counterparts or the environment by chemical signaling which is central to many biological processes. Examples range from bacteria or sperm that bias their motion in response to an external chemical gradient, to collective cell migration in response to a self-generated gradient. Here, in a purely physicochemical system based on selfpropelling oil droplets, we report a novel mechanism of dynamical arrest in active emulsions: swimmers are caged between each other's trails of secreted chemicals. We explore this mechanism quantitatively both on the scale of individual agent-trail collisions as well as on the collective scale where the transition to caging happens as a result of autochemotactic interactions.
\end{abstract}

\footnotetext{
${ }^{1}$ This chapter is published in Hokmabad, B. V., Saha, S., Agudo-Canalejo, J., Golestanian, R. and Maass, C. C. (2020). Quantitative characterization of chemorepulsive alignment-induced interactions in active emulsions. arXiv preprint arXiv:2012.05170. B. V. H. designed the research, performed experiments and data analyses and wrote the manuscript. S. S. performed theoretical modeling, data analyses and wrote the manuscript. J. A. performed theoretical modeling, data analyses and wrote the manuscript. R. G. supervised the project and wrote the manuscript. C. C. M. designed the research, performed data analyses, supervised the project, and wrote the manuscript.
} 
Chemorepulsive alignment-induced interactions in active emulsions

\subsection{Introduction}

Motile micro-organisms have evolved to sense their environment and react to it, e.g. by reorientation. They sense the gradients of an external stimulus or a field in their surroundings and migrate up or down that gradient; a behavioral response called taxis. If the gradient is formed in the concentration field of a chemical species, and the organism responds to it in the form of a change in their propulsion directionality/strategy, they have shown chemotaxis.

In single cellular organisms, chemotaxis guides many processes like colony migration [211] or biofilm formation (cf. Dictyostelium or Physarum). The response of an organism to a chemical gradient that is produced by the microorganisms themselves, is called autochemotaxis. Whether the chemical species is a chemoattractant or a chemorepellent the system displays positive or negative autochemotaxis, respectively. Existing studies are usually based on attractive signaling; however, repulsive signaling is also of practical importance, e.g. if a colony wants to explore space efficiently by mutual avoidance.

Autochemotaxis in colonies of living organisms causes complex behavior governed by an interplay between physical effects and biological processes $[212,213]$. One way to understand the underlying mechanism for the emergence of these complex behaviors is to untangle the biological effects and the physical mechanisms. To this end, the rapidly growing field of artificial active matter is attempting to design and develop synthetic micro-swimmers that can mimic for example chemotaxis strategies using principles of non-equilibrium physics. Self-phoretic particles are the broadly-studied example of artificial micro-swimmers that locally generate a chemical gradient and drift in this self-made gradient. It has been shown that suspensions of these particles exhibit non-trivial dynamics influenced by autochemotaxis [84, 86, 87, 214, 215]. We have previously demonstrated that self-propelling droplets [67] can be used as a model system for repulsive chemical signalling $[89,216]$. The droplets leave behind a trace of 'used fuel' which acts as a chemorepellent to other droplets, where the the motion of such a droplet is affected by the previous passage of another droplet. The phenomenology of the system has similarities to the collective chemotaxis of trail-following bacteria [90, 217 , expect that the interactions with the slow chemicals are repulsive rather than attractive.

In this study, we present a quantitative analysis of individual 'delayed collisions' and compare it to an analytical model based on time lag, angle of incidence and chemical 

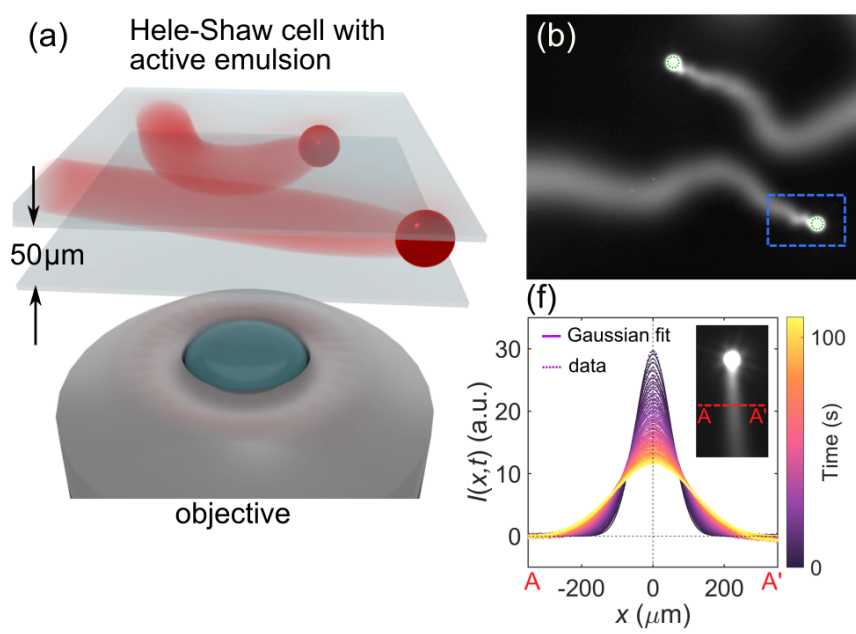

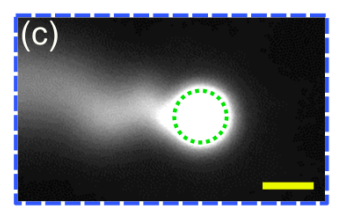

(d)
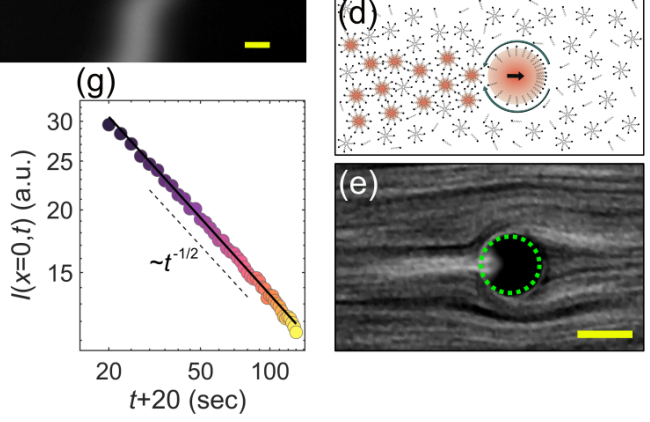

Figure 5.1 - Visualization of the chemical trail. (a) Schematic of the experimental setup for fluorescent microscopy of the filled micelle trail. (b) A micrograph of the chemical trails made by droplets under fluorescent microscopy. We used a higher surfactant concentration (15 wt.\%) to increase the solubilization rate and thereby the trail fluorescence. (c) Zoomed view of the panel (b). A $A^{\prime}$ is the cross-section at which the intensity profile was quantified. (d) The schematic for the propulsion mechanism of the droplet. The black arrow shows the direction of motion. (e) The flow field generated by Marangoni flow at the droplet interface visualized via streak lines of $0.5 \mu \mathrm{m}$ tracer particles. (f) The time evolution of light intensity profiles along $A A^{\prime}$ superimposed with the Gaussian fits. (g) The peak values of intensity profiles versus time. To account for the initial shape of the trail intensity profile, We model it with a linear chemical source that was released and started diffusing 20 seconds (obtained from fitting) before the passage of the droplet (see Methods). Scale bars show $50 \mu \mathrm{m}$.

coupling strength. We show that these parameters determine whether a droplet can go through the trail laid out by another active droplet or bounce back from it. In the second part of the study, we demonstrate how individual binary collisions lead to a novel state of autochemoctactic arrest in swimmer ensembles, a kind of 'history caging', where droplets are temporarily trapped in an evolving network of repulsive trails.

\subsection{Methods and general swimming behaviour}

Unless noted otherwise, all experiments were done in microfluidic cells using a quasi 2D Hele-Shaw geometry, with a typical cell area of $75 \times 50 \mathrm{~mm}^{2}$ and height of $50 \mu \mathrm{m}$ and observed using bright field or fluorescent microscopy. The active emulsions we used consisted of microdroplets of the oil CB15 with a diameter $a_{\text {drop }}=50 \mu \mathrm{m}$ placed, at low number densities, in an aqueous, supramicellar solution of the surfactant TTAB (at 5 wt.\%, unless otherwise stated, with $\mathrm{CMC}=0.13 \mathrm{wt} . \%$ ). If the surfactant concentration 
in the bulk medium exceeds a threshold value the droplet spontaneously undergoes a dynamical instability which breaks the symmetry and propels the droplet at a speed $V \approx 25 \mu \mathrm{ms}^{-1}$. The underlying propulsion mechanism [13,67] can be summarized as follows: The oil droplets gradually solubilize, with oil molecules migrating into surfactant micelles Since the interfacial surfactant density is coupled to the respective local densities of oil-filled and empty micelles, the trail of oil-filled micelles in the wake of a moving droplet locally increases the interfacial tension (Fig. 5.1 d), thus creating a self-sustaining Marangoni stress at the interface that pushes the droplet forward.

To visualize the chemical trail we added the hydrophobic fluorescent dye NileRed to the oil phase [102]. As the dye co-migrates with the oil into the micelles, we can obtain and quantify the distribution of oil field micelles from fluorescent microscopy (Fig. $5.1 \mathrm{a}, \mathrm{b}$ ). Fig. $5.1 \mathrm{c}$, d and e respectively, present the zoomed-in view of a fluorescent image, the schematic of the propulsion mechanism, and the streak lines of tracer particles that visualize the flow generated by the droplet (in droplet reference frame). We note that, for illustration purposes, the micrographs in Fig. 5.1 b,c and Fig. 5.5 a were recorded at a higher surfactant concentration of 15 wt. $\%$ to increase the solubilization rate and thereby the trail fluorescence. However, a lower concentration yields very ballistic droplet motion and long cruising ranges and is therefore preferable in quantitative experiments. To model the trail diffusion, we approximate the droplet as a moving point source emitting chemorepellent at a constant rate. Accordingly, the chemorepellent profile along a line perpendicular to the trail (e.g. $\mathrm{AA}^{\prime}$ in Fig. 5.1 f,inset), represented by the fluorescence intensity, should be Gaussian with a peak height scaling with $t^{-1 / 2}$. We have plotted Gaussian fits of the time dependent recorded intensity along $\mathrm{AA}^{\prime}$, as well as the respective amplitudes from the fits in Fig. 5.1 e,f, with excellent agreement, validating both the Gaussian distribution and $t^{-1 / 2}$ decay, and yielding a diffusion constant for filled micelles $D_{\mathrm{fm}}=52.5 \mu \mathrm{m}^{2} / \mathrm{s}$. This is consistent with our observations from previous studies [89] and common literature values for micellar diffusion (for details of the fitting please see Supp. Mat.). Based on this highly regular behaviour, we can in the following extract a chemical gradient map for the entire experiment by tracking the motion of all droplets in the system and treating them as chemorepellent point sources, even without recording micellar fluorescence. Using the experimentally-obtained quantities we can estimate the diffusive time scale of the trail decay: $\tau_{\text {diff. }}=a_{\text {drop }}^{2} / D_{\mathrm{fm}} \approx 24 \mathrm{~s}$, which is quite long due to the large size of the filled micelles $(\approx 5 \mathrm{~nm})$, and remarkably longer than the droplet's advective time scale $a_{\mathrm{drop}} / V \approx 2$ s. Such long-living chemical gradients can change the chemical potential 
landscape and influence the propulsion dynamics of other droplets even without direct hydrodynamic interaction. Beyond the advective timescale, we can therefore model droplet-trail interactions by a Chemically Active Polar Particle model approach [218], as follows.

\subsection{Chemically Active Polar Particle model}

The fore-aft flow at the interface is determined by the gradient in the local concentration of the empty micelles. We assume that, in the steady swimming state, the fore-aft asymmetry of the droplet is stably maintained and thus the swimming droplet can be modelled as a chemically-active polar particle [218, 219]. Thus, when a droplet encounters a trail, it experiences an effective torque governed by a coupling constant $\Omega$, and an effective force with coupling $\alpha$. We note that the general theory of chemicallyactive polar particles also allows for an effective force projected along the axis of the particle, with a third coupling constant, but we will neglect this possibility here as the data can be explained within the more minimal model with two coupling constants.

We define droplets to have a position $\boldsymbol{r}$ and orientation $\boldsymbol{n}$ and to move in the $(x, y)$ plane. The Langevin dynamics for $\boldsymbol{r}$ and $\boldsymbol{n}$ are as follows [218]:

$$
\begin{aligned}
\dot{\boldsymbol{r}} & =V_{0} \boldsymbol{n}-\alpha \boldsymbol{\nabla} c+\sqrt{2 D_{t}} \boldsymbol{\xi}_{t}, \\
\dot{\boldsymbol{n}} & =\Omega \boldsymbol{n} \times(\boldsymbol{n} \times \boldsymbol{\nabla} c)+\sqrt{2 D_{r}} \boldsymbol{n} \times \boldsymbol{\xi}_{r},
\end{aligned}
$$

where $\boldsymbol{\xi}_{t}$ and $\boldsymbol{\xi}_{r}$ represent Gaussian distributed translational and rotational noise with unit strength, and $D_{t}$ and $D_{r}$ are the corresponding translational and rotational diffusion coefficients of the droplet. Analysis of the mean squared displacement of the droplets in dilute conditions reveals ballistic trajectories over periods larger than $100 \mathrm{~s}$ (see Fig. 5.5(b)), suggesting that Brownian translational diffusion is negligible, and that the rotational diffusion coefficient is also small, with an upper bound of $D_{r}=0.01$ $\mathrm{rad}^{2} / \mathrm{s}$ corresponding to persistence lengths larger than $V_{0} / D_{r} \approx 2.5 \mathrm{~mm}$.

We model the micelle trail as a chemical field $c$ with Gaussian profile perpendicular to the direction of motion, with a width that depends on the time elapsed since the preceding droplet moved away from the point of interest. Without loss of generality we assume the preceding droplet to propel along $\boldsymbol{x}$, so that the chemical field is given by

$$
c(y)=\frac{c_{0}}{\sqrt{4 \pi D_{\mathrm{fm}} \Delta t}} \exp \left(-\frac{y^{2}}{4 D_{\mathrm{fm}} \Delta t}\right),
$$


where $D_{\text {fm }}$ is the diffusion coefficient of the (filled) micelles. Because the diffusion of the micelles is slow compared to the propulsion velocity of the droplets $\left(D_{\mathrm{fm}} /\left(\Delta t V_{0}^{2}\right) \ll 1\right)$, we can neglect the $x$-dependence of the concentration profile, as well as its timedependence, so that we can use (5.3) to describe the interaction of the incoming droplet with the trail.

We thus solve the following deterministic equations in 2D for the incoming droplet's position $(x, y)$ and orientation $\theta$, which is defined as the angle between $\boldsymbol{n}$ and the $x$-axis (see Fig. $5.2 \mathrm{~d}$ ):

$$
\begin{aligned}
\dot{x} & =V_{0} \cos \theta \\
\dot{y} & =V_{0} \sin \theta+\frac{\alpha c_{0} y}{\sqrt{2 \pi}\left(2 D_{\mathrm{fm}} \Delta t\right)^{3 / 2}} \exp \left(-\frac{y^{2}}{4 D_{\mathrm{fm}} \Delta t}\right) \\
\dot{\theta} & =-\frac{\Omega c_{0} y}{\sqrt{2 \pi}\left(2 D_{\mathrm{fm}} \Delta t\right)^{3 / 2}} \exp \left(-\frac{y^{2}}{4 D_{\mathrm{fm}} \Delta t}\right) \cos \theta
\end{aligned}
$$

\subsection{Experimental quantitative analysis of droplet- trail interactions in $2 \mathrm{D}$}

We experimentally studied individual droplet-trail interactions by placing droplets at low number densities in aqueous surfactant solutions in shallow Hele-Shaw microfluidic reservoirs. We recorded and analysed the droplet trajectories via video microscopy and mined the data for trail interactions (see Supp. Mat., section 5.10.3 for criteria).

Fig. 5.2 shows examples of crossing and reflecting interactions and the extracted data for the following droplet's signed trail distance $d$, speed $V$ and orientation $\theta$ (as shown in panels e and $\mathrm{f}$ for the interactions in $\mathrm{b}$ and $\mathrm{c}$ ).

Since our model assumes the preceding droplet to move in a pristine medium with isotropic chemical field, we selected interactions where its motion that can locally be well approximated by a straight line (red trajectories in Fig. $5.2 \mathrm{a}, \mathrm{b}$ ), and where we assume the chemical gradient in the trail to evolve according to Eqn. 5.3.

The droplet approaches the trail at an incident angle $\theta_{\text {inc }}$ with respect to the first trajectory (the blue trajectory in Fig. 5.2 a). An interaction starts and ends when the distance $|d|$ between the droplet and the trail falls below a threshold value $d_{\max }=220 \mu \mathrm{m}$. We identify the points of intersection (green points in a and b), or, for reflection, closest approach on each trajectory, and define the time lag $\Delta t$ as the interval between each droplet passing these points, and the time origin $t_{0}$ as the respective 

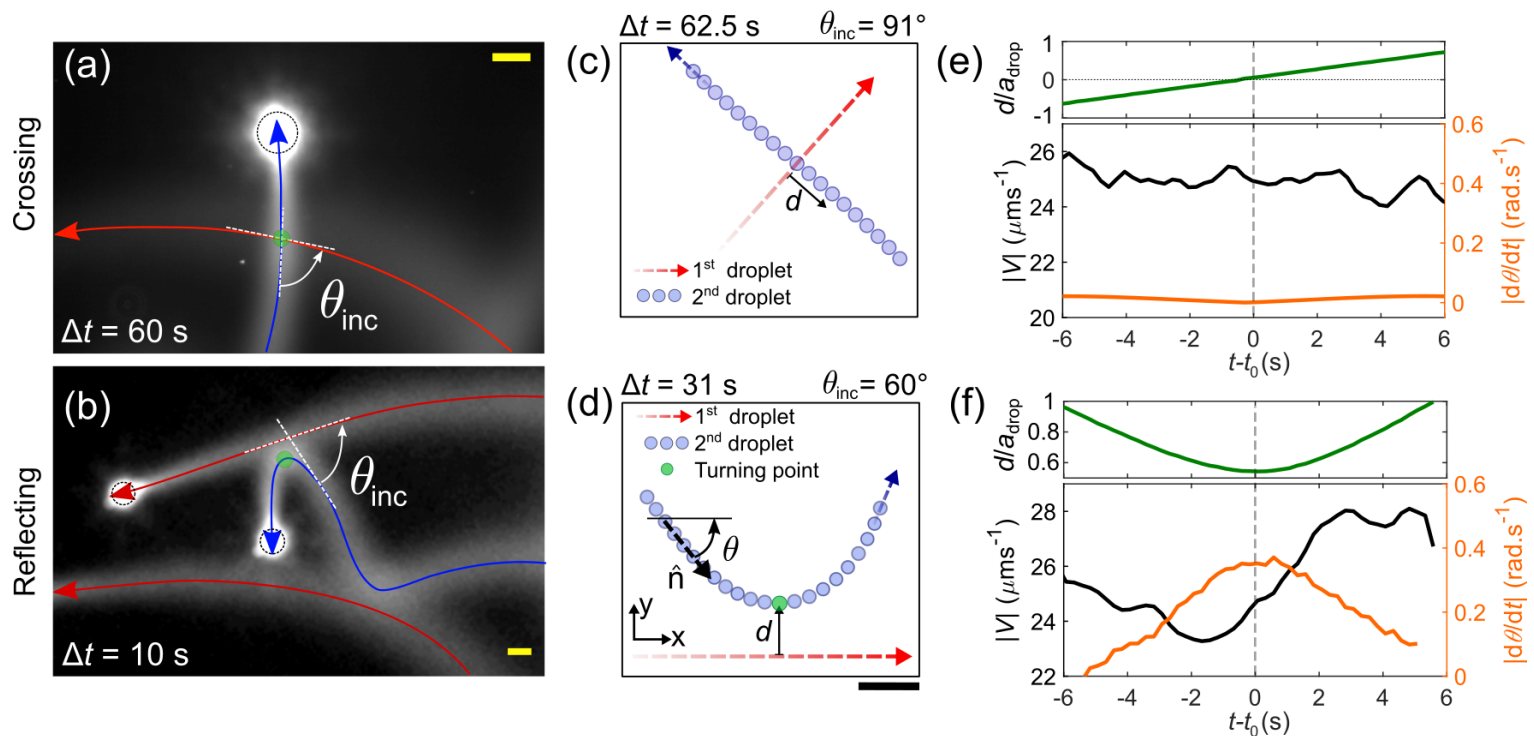

Figure 5.2 - Autochemotactic interaction between droplets. (a) The fluorescent micrographs of crossing and reflecting interactions. The red trajectory corresponds to the first passing droplet (secreting the trail) and the blue trajectory corresponds to the second droplet. (c) and (d) data from bright field microscopy, typical trajectories of crossing and reflecting interactions. (e) and (f) Plots of distance, swimming speed and rotation rate (angular velocity) for the interactions in (c) and (e), respectively. All scale bars are $50 \mu \mathrm{m}$.

point in time for the following droplet. We note that for non-specular reflections with $\alpha_{0} \neq 0$ (Eqn. 5.2) the time of maximum rotation rate, $t_{\mathrm{turn}}=|\mathrm{d} \theta / \mathrm{dt}|_{\max }$, should be slightly delayed with respect to the time of closest approach.

We observe that the probability of crossing versus reflection depends both on $\theta_{\text {inc }}$ - for a shallow angle of incidence, the required turning rate for reflection is lower and on $\Delta t$, which determines the gradient strength (Eqn. 5.3). We illustrate this in Fig. 5.2 a,b: we observe, for similar $\theta_{\text {inc }}$, a transition from reflection to crossing with increasing $\Delta t$. As shown in panels (d) and (f), there is also evidence of non-specular reflection, as well as the following droplet slowing down and speeding up around $t_{0}$, suggesting a negative $\alpha_{0}$.

To derive a phase diagram for crossing and reflection in $\left(\Delta t, \theta_{\text {inc }}\right)$ space, we will now proceed to a quantitative estimate of the model's parameters by numerical fits to our recorded data. 


\subsection{Fits of model and experiment droplet-trail in- teractions}

We fit the theoretical trajectories obtained from numerical solution of (5.3-5.6) to the experimental trajectories in order to obtain estimates for the two unknown coupling constants $\Omega c_{0}$ and $\alpha c_{0}$, which were used as fitting parameters. For the diffusion coefficient of the micelles, we used the measured value $D_{\mathrm{fm}}=52.5 \mu \mathrm{m}^{2} / \mathrm{s}$. The droplet velocity $v$ was estimated as the average velocity in the given trajectory, and the experimentally-measured time lag $\Delta t$ was used as an input. Initial conditions for the time evolution of $(x(t), y(t), \theta(t))$ were obtained from the data as described in the previous section: $y(0) \approx 200 \mu \mathrm{m}$ corresponds to the initial value of the signed distance $d_{\max }, \theta(0)$ to the incidence angle, and $x(0)=0$ without loss of generality.

For our analysis of the fits, we focused on sharp reflection events with $\theta_{\text {inc }}>60^{\circ}$, for which our minimal model of the trail as a static Gaussian with constant width is best justified (for low incidence angles, interactions occur over longer times and a wider range of $x$-values, which may affect the validity of our approximation and introduce differences between parallel and antiparallel reflection events). The 54 reflection events were fitted and ordered from best to worst fit according to the fit error. By analyzing the median values of $\Omega c_{0}$ and $\alpha c_{0}$ calculated from the $n$ best fits as a function of $n$, we found that the median values stabilize at $\Omega c_{0} \approx 7 \cdot 10^{3} \mu \mathrm{m}^{2} / \mathrm{s}$ and $\alpha c_{0} \approx 3 \cdot 10^{4}$ $\mu \mathrm{m}^{3} / \mathrm{s}$ between $n \approx 20$ and $n \approx 40$. Smaller $n$ values are susceptible to the noise due to small number statistics, whereas for larger $n$ we would include bad fits that skew the distribution, presumably corresponding to non-ideal trajectories (e.g. interactions with curved trails or measurement artifacts due to global drift in the chamber). Details can be found in Fig. 5.10 of the Supplement.

Examples of the fits are shown in Fig. 5.3 for three experimental trajectories. Here, blue lines correspond to the actual fit to the given trajectory, whereas red lines correspond to the theoretical prediction using the values $\Omega c_{0}=7 \cdot 10^{3} \mu \mathrm{m}^{2} / \mathrm{s}$ and $\alpha c_{0}=3 \cdot 10^{4} \mu \mathrm{m}^{3} / \mathrm{s}$. The characteristic features of the evolution of the position, velocity, and angular velocity with time are well recapitulated by the model, both for each particular fit and when using the median values. Importantly, $\Omega>0$ implies that the droplet reorients to point away from the trail, whereas $\alpha>0$ implies that the droplet is also directly repelled by the trail. While we found that the trajectory shapes are most sensitive to changes in $\Omega$, which is the key parameter governing the interaction, the presence of a positive $\alpha$ is essential in order to correctly capture the time evolution 

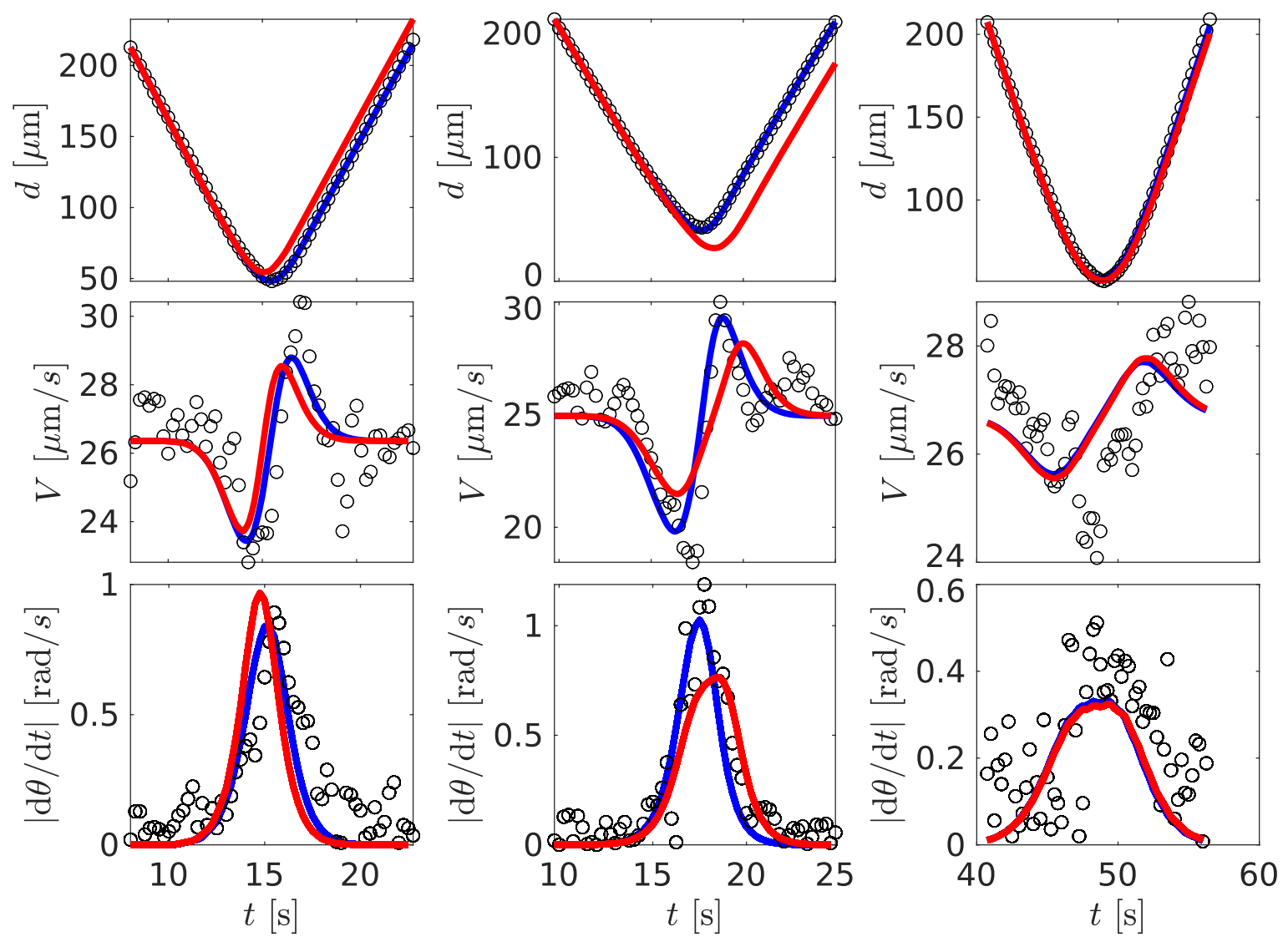

Figure 5.3 - Three example fits of the chemically-active polar particle theory to experimental trajectories, where each column corresponds to a different trajectory. Two fit parameters $\left(\Omega c_{0}\right.$ and $\left.\alpha c_{0}\right)$ were adjusted. The blue lines correspond to the resulting best fit for the given trajectory, whereas the red lines are the theoretically predicted trajectories using the median values of all fits analyzed, $\Omega c_{0}=7 \cdot 10^{3} \mu \mathrm{m}^{2} / \mathrm{s}$ and $\alpha c_{0}=3 \cdot 10^{4} \mu \mathrm{m}^{3} / \mathrm{s}$. 


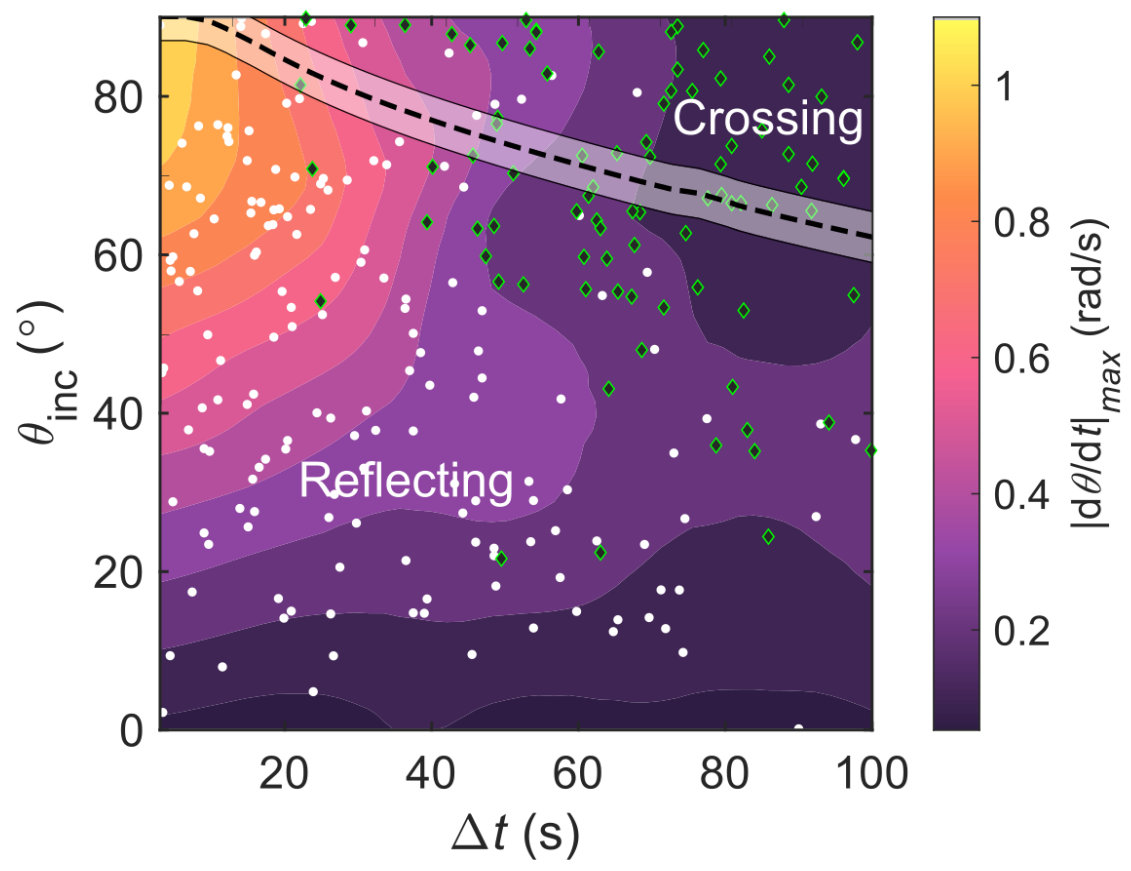

Figure 5.4 - Phase diagram of autochemotactic interactions. The colormap represents the maximum rotation rate during the particle-trail interaction $|\mathrm{d} \theta / \mathrm{dt}|_{\max }$ as obtained by fitting to the white (reflection events) and green (crossing events) points. The dashed line is the boundary between the crossing and reflecting events predicted by the theoretical model (including the uncertainty caused by rotational diffusion).

of the droplet velocity (second row in Fig. 5.3), which decreases before the turning point and increases after it.

\subsection{Phase diagram using results of the fit}

We determined $\theta_{\text {inc }}$ and $\Delta t$ for all selected interactions and plotted them in the phase diagram in Fig. 5.4 a, with reflections marked by white and crossings by black data points (164 reflection and 90 crossing events). We note that we do not distinguish between parallel and antiparallel interactions - for $\theta_{\text {inc }}>90^{\circ}$, we convert to $180^{\circ}-\theta_{\text {inc }}$. The background colour map interpolates the maximum turning rate measured for reflections. We can confirm two key features: For one, crossing is more likely for decreasing $\nabla c$, i.e. increasing $\Delta t$. Second, for increasing $\theta_{\text {inc }}$, i.e. sharper reorientations, reflection requires a higher turning rate, mediated by $\Omega|\nabla c|$, such that crossing is also more likely for increasing $\theta_{\text {inc }}$.

We can construct a theoretical phase diagram by using the median values of $\Omega c_{0}$ 
and $\alpha c_{0}$ obtained in the previous section. The separatrix (dashed white line) between crossing and reflecting trajectories, which coincides with those trajectories for which the turning point is exactly at the center of the trail $(y=0)$, reproduces the salient features of the experimental observations. The small amount of rotational noise present in the system can cause some uncertainty in the location of the separatrix, which can be estimated by considering the standard deviation of the orientation while the incoming droplet approaches the trail, which is $\delta \theta \approx \sqrt{2 D_{r} T}$, where the approach time is $T \approx d_{\max } /\left(V_{0} \sin \theta_{\text {inc }}\right)$ and the upper bound of the rotational diffusion coefficient $D_{r} \approx 0.01 \mathrm{rad}^{2} / \mathrm{s}$ is used. The strongest deviations between the theoretical prediction and experimental results are observed at low $\theta_{\text {inc }}$ and large $\Delta t$, in which case both crossing and reflecting trajectories are seen to coexist experimentally. We expect that in this region higher order effects, such as the time-dependence and space-dependence of the trail width (which breaks the symmetry between parallel and antiparallel trajectories) will play a role.

\subsection{Collective dynamics governed by autochemo- tactic interactions: history caging}

In a more crowded system, moving droplets trace out a network of diffusing trails, leading to frequent reorientations, and causing a dynamically evolving chemical potential landscape. This causes droplets to get transiently trapped in the interstitial spaces of the trail network.

To study the collective dynamics in $2 \mathrm{D}$, we placed suspensions of swimmers at number densities between $n \approx 0.025$ and $8.58 / \mathrm{mm}^{2}$ in a quasi-2D Hele-Shaw cell and recorded their trajectories for long times $(\approx 5 \mathrm{~min})$. We first illustrate the trapping behaviour by snapshots from a fluorescently dyed sample in Fig. 5.5 a. We follow one swimmer by marking its trajectory in cyan. The first image was chosen shortly after the addition of the droplets, hence, the experiment starts with almost no secreted trails. Initially, all droplets move persistently, and their first reorientation is a result of the first encounter with a trail. As they travel the secreted trails gradually form a network of trails. A potential landscape based on $\nabla c$, evolves, with local minima (dark regions) between the trails. The swimmers get dynamically arrested by multiple reflections at the walls of these transient cages - escape is possible only when $\nabla c$ has decreased sufficiently at the boundaries or when the chemical buildup caused by the 
droplet itself forces it out of the cage.

Fig. $5.5 \mathrm{~b}$ shows, for increasing number densities, the mean squared displacement profiles obtained by ensemble averaging over the trajectories,

$$
\operatorname{MSD}(t)=\frac{1}{N} \sum_{i=0}^{N}\left(\mathbf{r}_{i}(t)-\mathbf{r}_{i}\left(t_{0}\right)\right)^{2}
$$

where $t_{0}$ is the starting moment of the experiment and $N$ the number of droplets. For any number density, droplets initially undergo ballistic propulsion (MSD $\sim \mathrm{t}^{2}$ ). For a single droplet (no interactions), we do not see a transition to diffusive scaling. For intermediate number densities, we observe a change in the slope of the MSD profiles, which is associated with the reorientations caused by the autochemotactic interactions. At large number densities (150 droplets and more), after a short ballistic period, the MSD reaches a plateau. This plateau is reminiscent of the caging signature of in the MSD for colloidal glasses [220], however, here the caging is caused by trail-droplet instead of direct droplet-droplet collisions and is therefore observed at much lower volume fractions $\left(\phi_{\text {droplets }} \approx 10^{-2}\right.$, cf. [221]). For more crowded systems, the crossover to caging happens earlier, the lifetime of the cage is longer, and the cage size is smaller. The plateau is followed by a crossover to a third, subballistic regime caused be consecutive caging events. We have illustrated this for an example trajectory in Fig. $5.5 \mathrm{c}$, for a droplet that undergoes three caging events in a very crowded system (300 droplets). The droplet trajectory is mapped by circles colour coded by time in the experiment. To highlight the cage formation by spatiotemporal density fluctuations, we plotted in the image background, using the same colour code, all droplet positions recorded within a window of $d<220 \mu \mathrm{m}$ and $0<\Delta t<50$ s around the current droplet position. Entering an area with increased density, the droplet reorients frequently, staying in place until it is ejected by the chemical buildup into a less populated space, where it proceeds ballistically until it encounters the next high-density location ('cage escape'). Since ballistic runs are uncorrelated, the long-time dynamics presumably correspond to a Gaussian random walk, however, it is not feasible to reliably quantify the respective exponent in the MSD due to the limited lifetime of the droplets and the finite size of the experimental cell. 

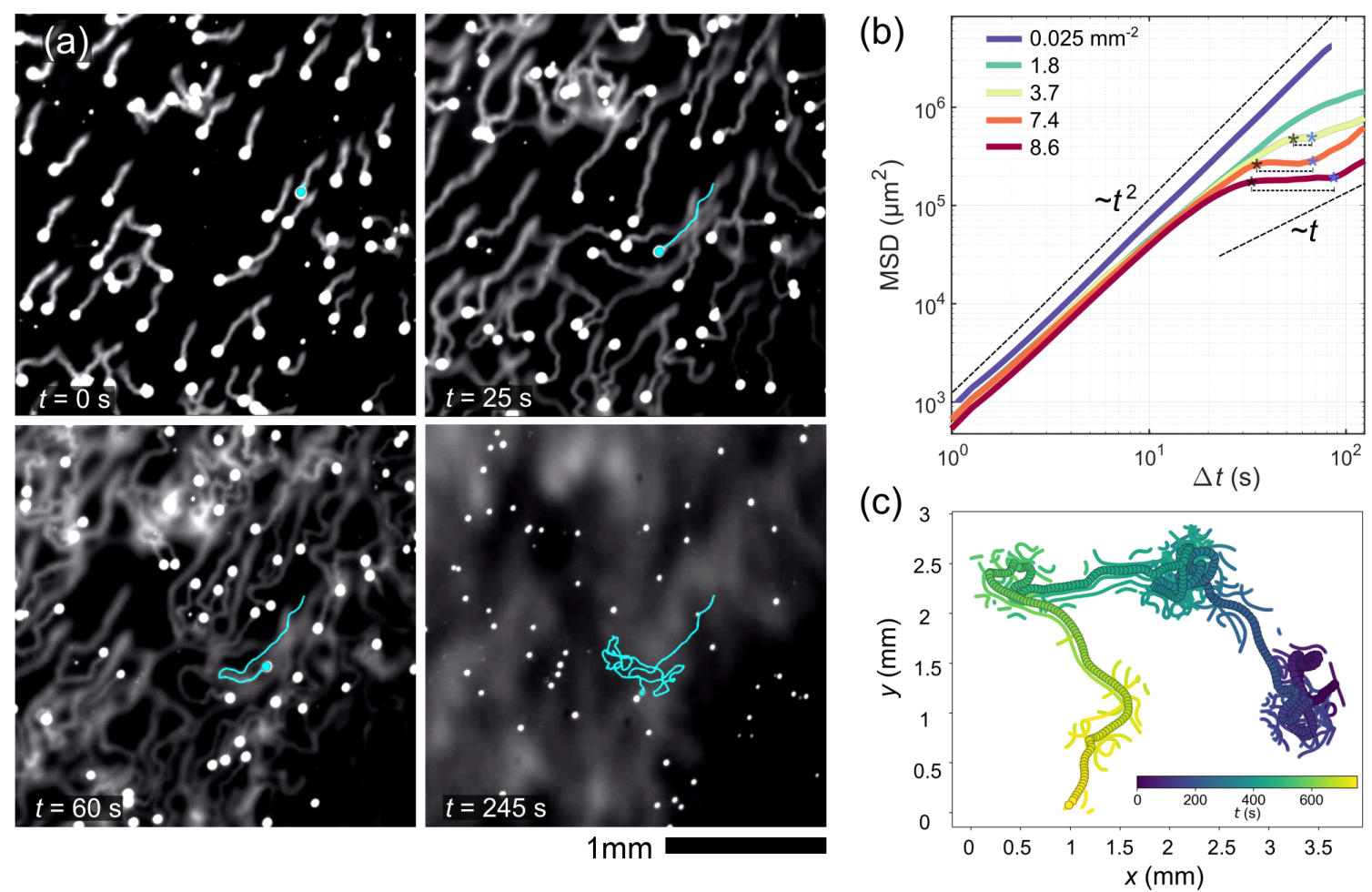

Figure 5.5 - 2D caging. (a) Snapshots from fluorescent microscopy of an active emulsion in a Hele-Shaw cell (droplet size $50 \mathrm{\mu m}$ ). The experiment was conducted at high droplet solubilization rate to increase trail visibility. One sample trajectory is plotted to show the evolution from ballistic propulsion to a caging event. The scale bar is $1 \mathrm{~mm}$. (b) Mean squared displacement profiles for emulsions with different number densities. The $\star$ denotes the cross-over to the caging regime. The $\star$ denotes the cross-over to the third regime (cage-escape). (c) A typical trajectory $s(t)$ undergoing several caging and cage-escape events (thick symbols). Thin symbols: other droplets approaching within a spatiotemporal window of $d<220 \mu \mathrm{m}$ and $0<t_{0}-t<50 \mathrm{~s}$ around the current trajectory point $s\left(t_{0}\right)$ The colormap represents the time.

\subsection{Extending the system to 3D}

One intriguing feature to study is how far dimensionality matters in caging effects. While, in two dimensions, fully confining cages exist even for point-like particles and one-dimensionally parameterized trajectories, in three dimensions the cage would always have holes the droplet can escape through. Caging in $3 \mathrm{D}$ is therefore only possible owing to the finite volume of the diffusing trail.

To investigate caging in 3D, we studied droplet ensembles in unconfined, force free bulk media. We eliminated the effect of gravity, matching the density of the swimming droplets with the aqueous surfactant solution by adding heavy water, $\mathrm{D}_{2} \mathrm{O}$. We observed dyed droplets using a scanning light sheet fluorescence microscope over a 
Chemorepulsive alignment-induced interactions in active emulsions

volume of $3 \times 3 \times 3 \mathrm{~mm}^{3}$.

To validate the diffusive spreading of the trail in $3 \mathrm{D}$, we first captured the timedependent fluorescence in the trail of a droplet sedimenting under gravity along the light sheet normal, taken at a fixed sheet position in $z$ (Fig. 5.6 a). We extracted the fluorescence intensity profile $I(x, t)$ of a cross section of the trail along the line $\mathrm{AA}^{\prime}$ (Fig. 5.6 c), as shown in the false-colour sample image in Fig. $5.6 \mathrm{~b}$. The peak values $I_{\text {peak }}$ scale with $t^{-1 / 2}$ (inset), similar to the 2D behaviour analyzed in Fig. 5.1.

Next, to study the collective dynamics in 3D, we recorded the $3 \mathrm{D}$ trajectories of droplets in active emulsions over long times. Fig. $5.6 \mathrm{~d}$ illustrates the simultaneous tracking for an experiment at intermediate number density, $n=8 / \mathrm{mm}^{3}$, showing a $3 \mathrm{D}$ reconstruction of all droplet positions at an arbitrary time in the experiment, superimposed with trajectories for the droplets we were able to track for sufficiently long times $(\approx 5 \mathrm{~min})$. We have plotted typical trajectories for systems with different number densities in Fig. $5.6 \mathrm{e}-\mathrm{g}$. In the dilute case $\left(n=2 \mathrm{~mm}^{-3}\right)$, the trajectory is quite straight, while in the system with intermediate number density, the droplet experiences a few reorientation events. The trajectory for the dense system shows alternating quasi-ballistic and caged sections similar to the dynamics in 2D (Fig. 5.5 c).

In Fig. $5.6 \mathrm{~h}$, we plotted the mean squared displacement of a set of 3D trajectories of sufficient length extracted from the data sets used for Fig. 5.6 e-g. The signatures of caging can be observed in the form of plateaus in the MSD profiles (in particular for the $n=22 \mathrm{~mm}^{-3}$ case). These observations suggest that even in $3 \mathrm{D}$, the droplet can get trapped in an evolving chemical potential landscape created by the trails of other swimmers.

\subsection{Conclusion and outlook}

In conclusion, we used dilute active emulsions as artificial systems to explore the collective dynamics of active particles governed by negative autochemotactic interactions. Based on fluorescent imaging, we visualized and measured the diffusion coefficient of the filled micelle trails left in the wake of the droplets; a quantity necessary for theoretical modeling of the interactions. We quantified these autochemotactic interactions and showed that whether a droplet crosses or is reflected by a trail can be predicted by the time lag between droplet passages and the angle of incident.

We further explored the collective dynamics in active emulsions and observed a novel dynamical arrest mechanism: transient autochemotactic caging. We extended 
(a)

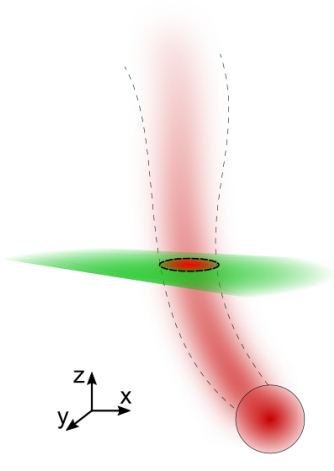

(b)

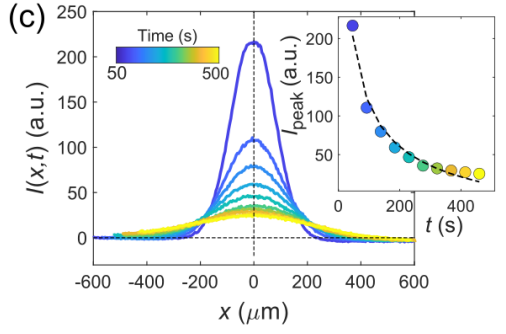

(e)

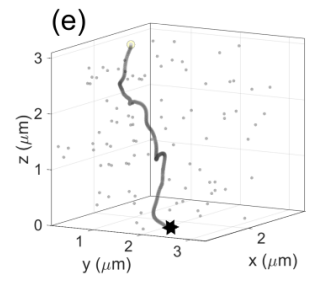

(f)

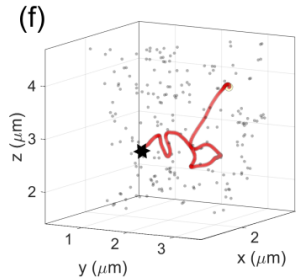

(g)

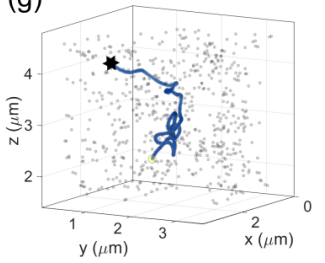

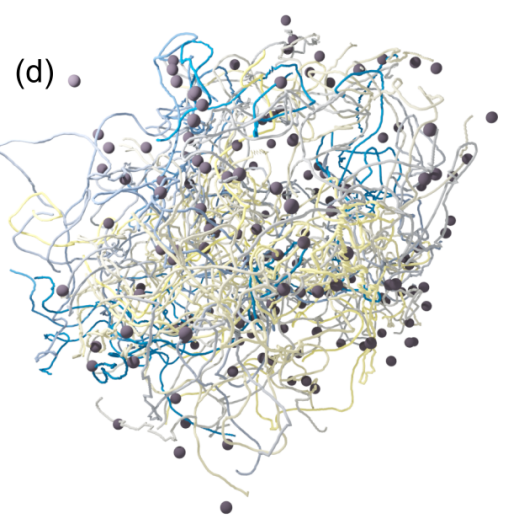

(h)

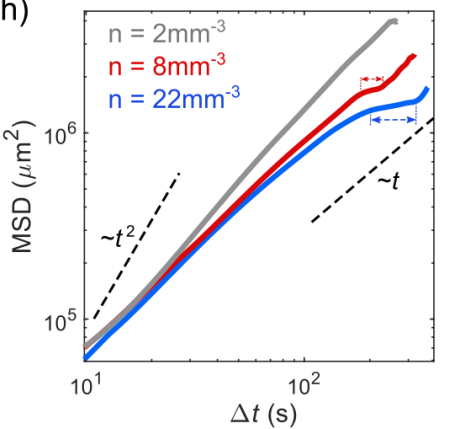

Figure 5.6 - Extending the system to 3D. (a) The schematic of trail visualization in 3D. The green sheet represents the laser light which scans the trail at different heights obtaining images like (b). (c) The temporal evolution of intensity profiles obtained by laser sheet measurements. The inset shows the peak values versus time. The dashed line is a fit to the data to show the scaling with $t^{-1 / 2}$. (d) The $3 D$ distribution of the droplets obtained by reconstruction of the scanned images superimposed with the trajectories. (e-g) Typical 3D trajectories of a droplet in emulsions with different number densities (2, 8 , and $22 / \mathrm{mm}^{3}$, respectively). The background shows the $3 D$ distribution of the droplets in the emulsion at an arbitrary time during the experiment. The ending points of the trajectories are denoted by the star symbol. (h) Mean squared displacement profiles for emulsions with different number densities.

our system to 3D and showed that caging also happens in unconfined suspensions of active droplets. Interestingly, caging already happens at significantly lower volume fractions $\left(\phi \approx 10^{-4}\right)$, compared to the $2 \mathrm{D}$ case. Apart from dimensional effects, one contributing factor might be that the droplet motion in 3D is not rectified by the cell boundaries and that reflection is therefore already effected by weaker gradients. This question, however, cannot be resolved yet without further quantitative modelling of the coupling parameters.

While it is in principle feasible to treat individual droplet-trail interactions including hydrodynamic feedback [222], the chemically-active polar particle model does a very good job in capturing essential features of the collision behaviour: Due to the slow 
(a)

Chemorepulsive alignment-induced interactions in active emulsions
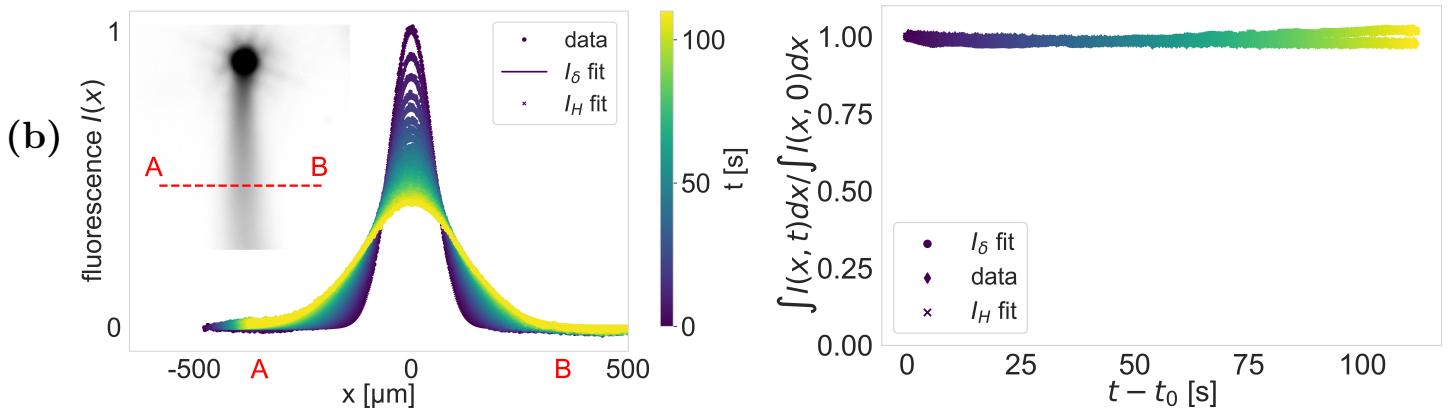

Figure 5.7 - Fluorescent emission in the trail of a Nile Red doped droplet. (a) Data vs. $I_{\delta}(x, t)((5.7))$ and $I_{H}(x, t)((5.9))$ fit models (b) The integrated fluorescence does not decay over time.

trail diffusion, our experiments show that reflection is still frequent after the flows caused by the leading droplet have decayed, and statistically the majority of observed interactions would happen when there is no advective contribution from the leading droplet. This makes the collective behaviour far more accessible to numerical and analytical modelling by using a similar simplified paradigm.

\subsection{Materials and Methods}

\subsubsection{Materials and characterisation}

Our oil phase consists of CB-15, an isotropic isomer of the common nematic liquid crystal oil 5CB. Monodisperse droplets (size approx. $50 \mu \mathrm{m}$, exact values to be added) are mass produced in microfluidic flow junctions. For the purpose of chemical quantification, we dissolved small amounts of the fluorescenct dye Nile Red in the oil phase. Nile Red does not fluoresce in water, such that we can assume the fluorescent intensity to stem from micelles filled with oil and co-migrating Nile Red. This assumption is supported by the diffusive spread of fluorescence in the trail mapped in Fig. 5.7. The swimming medium is $5 \mathrm{wt} \%$ aqueous solution of TTAB surfactant. For force free bulk measurements, the density of $5 \mathrm{CB}\left(\rho_{5 \mathrm{CB}}=1.05 \mathrm{~g} / \mathrm{cm}^{3}\right)$ was matched by appropriate heavy water substitution $\left(\rho_{\mathrm{D} 2 \mathrm{O}}=1.2 \mathrm{~g} / \mathrm{cm}^{3}\right)$. Microfluidic Hele-Shaw cells were made from the photoresist polymer SU8 by photolithography. 


\subsubsection{Micellar diffusion quantification by fluorescence}

We recorded fluorescent emission by videomicroscopy $\left(\lambda_{e x .}=545 \mathrm{~nm}, \lambda_{e m}=572\right)$ on an Olympus IX-73 at 4x magnification and 4 fps, using a $4 \mathrm{Mpx}$ Grasshopper camera. After rotation and rectification of the extracted image series, we extract profiles along a $y=$ const. image slice (line A-B in Fig. $5.7 \mathrm{a}$ ). We fit the profiles to the time and space dependent decay from a fluorescence quantity $c\left(x, t=t_{0}\right)=M_{0} \delta(0)$. $t_{0}$ is earlier than the actual moment of droplet passage, since the droplet is not a point source:

$$
\begin{aligned}
I_{\delta}(x, t) & =\frac{M_{0}}{\sqrt{4 \pi D_{\mathrm{fm}}\left(t-t_{0}\right)}} \exp \left(\frac{-x^{2}}{4 D_{\mathrm{fm}}\left(t-t_{0}\right)}\right), \\
M_{0} & =\int_{-\infty}^{\infty} I(x, 0) \mathrm{d} x
\end{aligned}
$$

We calculate the micellar diffusion coefficient via $\sigma^{2}=2 D_{\mathrm{fm}}\left(t-t_{0}\right)$. We note that a more precise model can be based on a decay from two step functions at a distance $d=50 \mu \mathrm{m}$, with $c(x, t=0)=c_{0}[H(x+d / 2)-H(x-d / 2)]$.

$$
I_{H}(x, t)=\frac{I_{0}}{2}\left[\operatorname{erf}\left(\frac{x+d / 2}{\sqrt{4 D_{\mathrm{fm}} t}}\right)-\operatorname{erf}\left(\frac{x-d / 2}{\sqrt{4 D_{\mathrm{fm}} t}}\right)\right]
$$

However, for our times of interest beyond the advective time scale the difference between the models is negligible (Fig. 5.7).

We note that, since the integrated fluorescence does not decay over time in Fig. 5.7 b, we do not have to account for possible bleaching effects in our analysis.

\subsubsection{Binary collisions: Image processing and data analysis}

After region of interest selection, background correction and binarisation, we extracted droplet coordinates via a contour search algorithm [223] combined with a blob size filter for each video frame. To rule out interactions by hydrodynamic entrainment, or direct droplet to droplet interactions, we filtered the resulting coordinate set to exclude droplets whose distance within the same frame are below a set threshold, as well as droplets too close to the cell boundaries. Droplet trajectories were extracted from the filtered set via a Crocker-Grier type algorithm, providing droplet coordinates $x, y$ and speed vectors $\mathbf{v}$ for each recorded timestep. We identified and analysed interactions as follows: for each pair of trajectories, we identified matching sections where the trajectory distance fell below a set threshold. Interactions that include a trajectory 
endpoint were excluded as we cannot guarantee these to be complete. Segment pairs $s_{1}, s_{2}$ were sorted by time. We assume the droplet creating $s_{1}$ to move freely, such that $s_{1}$ can be safely approximated by a straight line. We note that our swimmers' dynamics are persistent Brownian rather than strictly ballistic, however, the trajectory persistence length, as seen in Fig. 5.8, clearly exceeds the typical interaction length, so that our assumption of straight segments is reasonable. We identified the orientation $\hat{\mathbf{e}}_{1}$ of $s_{1}$ via a linear regression fit. If the standard deviation of this fit exceeded a certain value, the segment was considered to be too crooked, i.e. not relating to free motion, and the interaction was discarded. Fig. 5.8 shows trajectories from one experimental run with the numerically identified interactions marked in colour. We further discarded, by visual inspection, any interactions that were disturbed by multiple trail collisions. For segment $s_{2}$, we extracted the following quantities:

1. the time $\Delta t=t_{2}-t_{1}$ elapsed between the two points of closest trajectory approach, which we chose as the time delay of the interaction.

2. for each coordinate in $s_{2}$, the distance to the closest point in $s_{1}$, i.e. distance $d(t)$ of swimmer to trail over time. To mark crossing events, by convention, $d$ is signed via $\operatorname{sgn}(d)=\operatorname{sgn}\left(\mathbf{n}_{2} \times \hat{\mathbf{e}}_{1}\right)$, with $\mathbf{n}_{2}$ denoting the $s_{2}$ trajectory normal and $\times$ the $2 \mathrm{D}$ cross product.

3. from a projection of $\mathbf{v}_{2}$ on $\hat{\mathbf{e}}_{1}$, droplet speeds $v_{\|}$parallel and $v_{\perp}$ perpendicular to the trail, as well as the angle $\theta$ between $\mathbf{v}_{2}$ and $\hat{\mathbf{e}}_{1}$. To avoid discontinuities in $\theta$ due to $2 \pi$ periodicities, $\theta$ was calculated with respect to $-\hat{\mathbf{e}}_{1}$ if $\left\langle v_{\|}\right\rangle>0$.

\subsubsection{Laser sheet fluorescent microscopy}

The setup (Fig. 5.9) consists of an illumination unit (producing the thin laser sheet) and a detection unit (the camera and the objective) that are synchronized and translate vertically (in z-direction) capturing images at a frame size of $1 \mathrm{MP}$ in the $\mathrm{x}-\mathrm{y}$ plane at $4.15 \mu \mathrm{m} / \mathrm{px}$ resolution. Images are recorded at 150 frames per second, while the z-stages are driven by a sawtooth signal with an amplitude of $3 \mathrm{~mm}$ and a frequency of $0.7 \mathrm{~Hz}$, yielding a voxel size of $4.2 \times 4.2 \times 27 \mu^{3}$. The laser sheet's beam waist is $\approx 40 \mu \mathrm{m}$ thick. The scanned volume in the square cuvette we use is $3 \times 3 \times 3 \mathrm{~mm}^{3}$. Our samples consist of Nile Red doped droplets with a diameter of $50 \mu \mathrm{m}$ in density matched mixtures of $\mathrm{TTAB} / \mathrm{H}_{2} \mathrm{O} / \mathrm{D}_{2} \mathrm{O}$.

$3 \mathrm{D}$ droplet positions are reconstructed from binarized stacks of $z$-slices for each half period of the sawtooth signal. We extract fluorescent droplet contours for each 

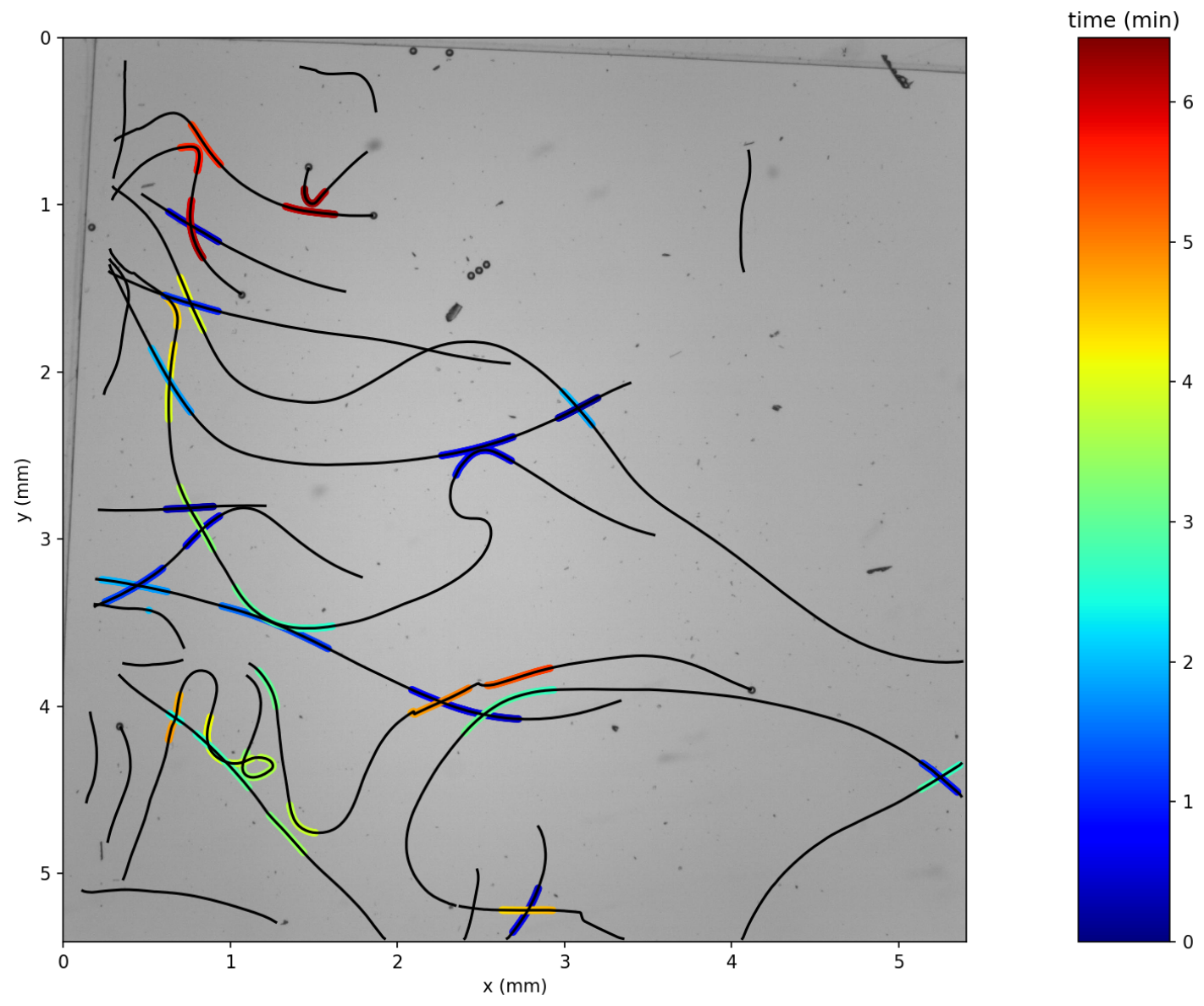

Figure 5.8 - Identification of collision events. 


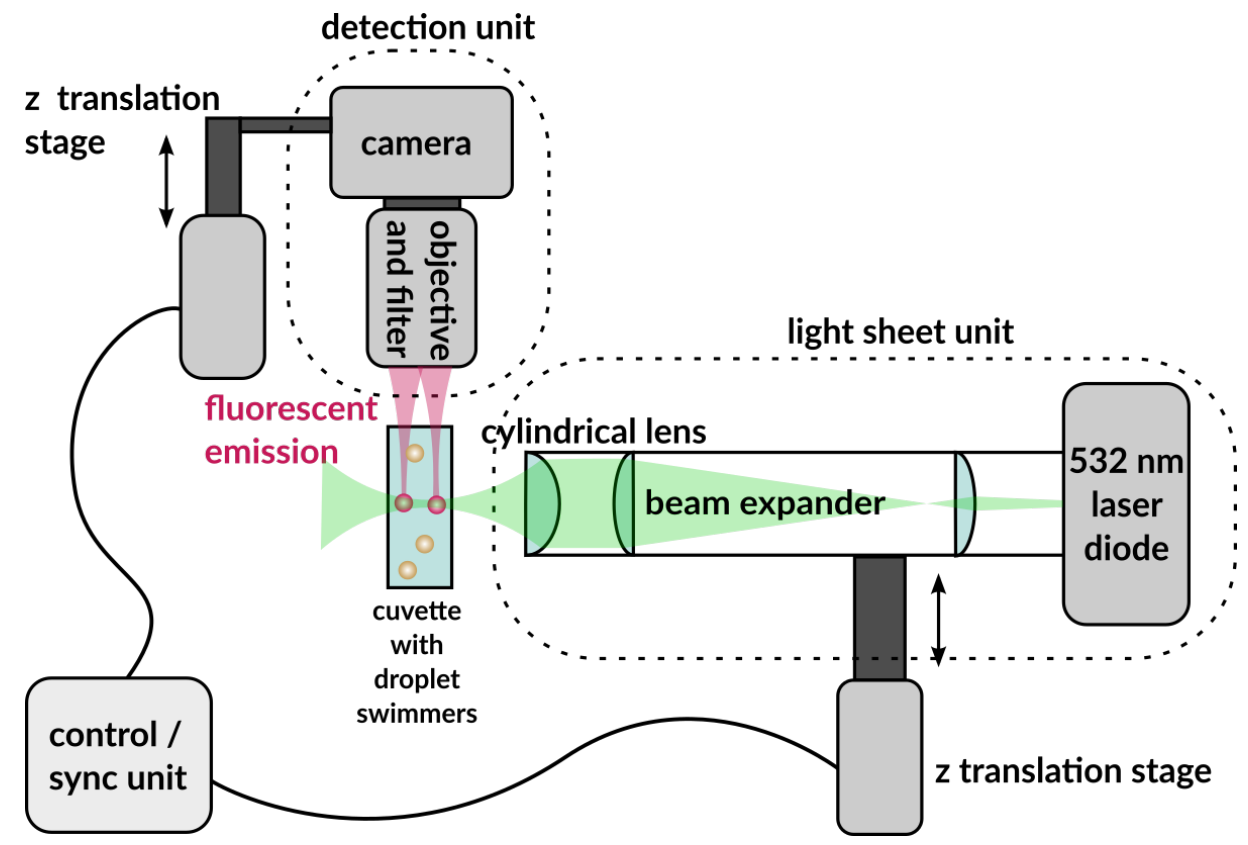

Figure 5.9 - Fluorescent light sheet microscope, schematic.

slice [223] and grouped contours associated with the same droplet within consecutive slices using a mean shift clustering algorithm [224, 225]. Time and $z$ position for each slice are calculated using timestamps provided by the camera and translation stage software interfaces, resulting in full $x y z t$ datasets. 

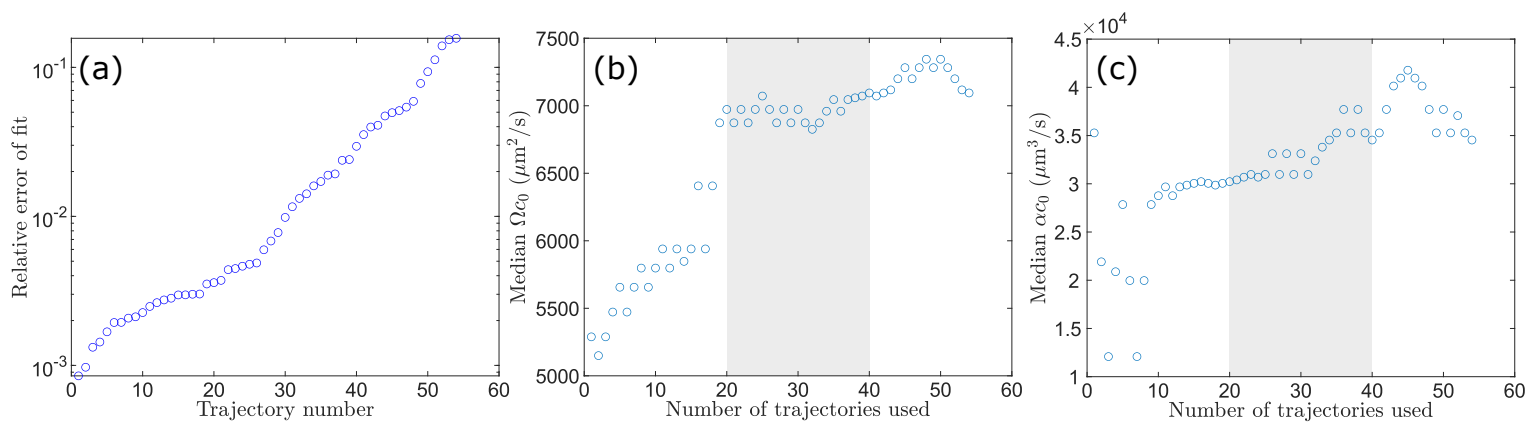

(d)

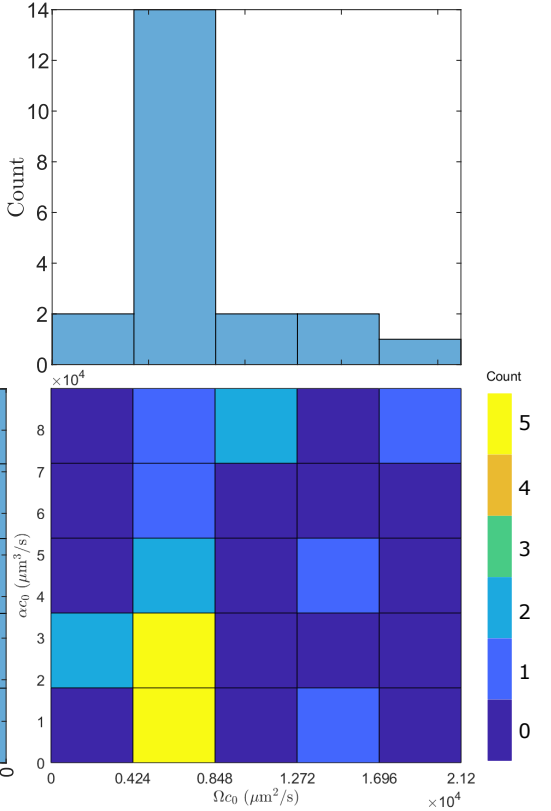

Figure 5.10 - Analysis of the fits to the 54 experimental reflection events with large incidence angle $\theta_{\text {inc }}>60^{\circ}$, using two fitting parameters $\Omega c_{0}$ and $\alpha c_{0}$. (a) The fits are ordered from best to worst according to the error of the fit. $(b, c)$ Median values of (b) $\Omega c_{0}$ and (c) $\alpha c_{0}$ as a function of the the number $n$ of trajectories used in the calculation of the median value. We observe a plateau at $\Omega c_{0} \approx 7 \cdot 10^{3} \mu \mathrm{m}^{2} / \mathrm{s}$ and $\alpha c_{0} \approx 3 \cdot 10^{4}$ $\mu \mathrm{m}^{3} / \mathrm{s}$ (grey band), when a sufficient number of trajectories is considered ( $n \gtrsim 20$ ) but the worst fits are left out $(n \lesssim 40)$. (d) As an example, we show the histogram for the values of $\Omega c_{0}$ and $\alpha c_{0}$ (two-dimensional, as well as projected along each dimension) when using the $n=21$ best fits, which corresponds to using only the fits with relative error $<4 \cdot 10^{-3}$. 



\title{
Chapter 6
}

\section{Spontaneously rotating clusters of active droplets: Collective dynamics governed by hydrodynamic interactions ${ }^{1}$}

\begin{abstract}
We report on the emergence of spontaneously rotating clusters within an active emulsion. The constituent self-propelling droplets sediment, self-organize into ordered planar clusters, and then hover over the bottom boundary exhibiting regular rotational dynamics. We trace back the origin of this rotation to the nonlinear dynamics of the individual droplets and demonstrate a novel symmetry breaking mechanism: helical propulsion dynamics for an isotropic active particle. The helical swimming regime is limited to a very narrow range of droplet activity between the straight and chaotic regimes. However, we show that by forming an ordered cluster, the droplets cooperatively suppress their chaotic dynamics, turning it into a steady rotational motion. We study the collective dynamics as a function of droplet activity and cluster size.
\end{abstract}

\footnotetext{
${ }^{1}$ This chapter is under preparation for publication. B. V. H. designed the research, performed experiments and data analyses and wrote the manuscript. Akinori Nishide, from Center for Exploratory Research, R\&D group, Hitachi Ltd., performed experiments and data analyses. Corinna Maass designed the research, performed data analyses, supervised the project, and wrote the manuscript.
} 
Spontaneously rotating clusters of active droplets

\subsection{Introduction}

Suspensions of colloidal particles have been shown to demonstrate a variety of collective behaviors when they are driven out of equilibrium. Whether driven externally [176, 226, 227 ] or internally $[78,79]$, the initially disordered suspensions tend to spontaneously form flocks and vortices or self-assemble into ordered crystal-like structures in the vicinity of a solid boundary. The majority of the studies on collective dynamics have focused on motility-induced phase separation (MIPS) where the particle velocity depends on the local concentration of its counterparts $[9,76,78]$. In these studies, mutual hydrodynamic interactions have been neglected. On the other hand, recently, a growing body of research has focused on collective behavior in active matter driven mainly by hydrodynamic interactions [228-231]. In particular, flow-induced selfassembly into ordered two-dimensional crystals has been reported in suspensions of Thiovulum majus bacteria [232] which attract their neighbors by generating a tornadolike flow field. Flow-induced phase separation has also been observed in suspensions of artificial active particles such as bottom-heavy Janus colloids [78] and active droplets [79, $80]$.

In this chapter, we study flow-induced self-assembly in active emulsions and report on the collective dynamics of swimming droplets that lead to a novel emergent behavior: close to a boundary, active droplets form ordered two-dimensional clusters that spontaneously undergo rotational motion. We show that the stability and dynamics of the clusters can be controlled by droplet activity and cluster size. To elucidate the underlying mechanism behind the rotational dynamics, we studied the individual swimming behavior in three dimensions and observed helical swimming which is a novel mechanism of spontaneous symmetry breaking for an isotropic active particle. Our experiments show that this scenario is limited to a very narrow activity range before the propulsion dynamics become chaotic. We discuss how forming an ordered cluster might stabilize the chaotic dynamics of individual droplets, thereby resulting in emergent rotational dynamics.

\subsection{Attractive hydrodynamic interaction between droplets}

We study active emulsions of oil droplets (CB15, radius $\left.R_{\mathrm{d}}=25 \mu \mathrm{m}\right)$ submerged in an aqueous supramicellar solution of surfactant TTAB $\left(c_{\mathrm{TTAB}}=6-20 \mathrm{wt}\right.$. $\%$, where 
$\mathrm{CMC}=0.13 \mathrm{wt} . \%)$. Due to the presence of the micelles, the oil droplets slowly dissolve by micellar solubilization. Hence, the system is out of equilibrium. Above a critical $c_{\text {TTAB }}$ the droplets undergo a dynamical instability and start to self-propel with a speed of $V \approx 25 \mu \mathrm{m} / \mathrm{s}$ (see Section 1.3 .3 and references $[13,58]$ ).

We used particle image velocimetry (PIV) to measure the external flow field generated by a droplet in a Hele-Shaw cell (height $\approx 2 R_{\mathrm{d}}$ ). Fig. 6.1 a shows that the magnitude of the interfacial velocity does not peak at the equator. Instead, the droplet generates an asymmetric flow that is stronger at its rear side, thereby showing a weak "pusher" flow in the far-field. This has also been observed in the numerical simulations of active droplets [58] as well as solid phoretic particles [57]. Thutupalli et al. [80] attributed this asymmetry to the balance between viscous and nematic stresses within the, in their case, liquid crystal droplet. Our experiments on isotropic droplets, however, show that this asymmetry originates from the complex transport of surfactant molecules in the external phase rather than the internal structure of the droplet.

To understand the mutual hydrodynamic interactions between droplets, we start from the simplest case: two droplets in a Hele-Shaw cell. As shown in Fig. 6.1 b,c, the two droplets attract each other, form a stable configuration, and continue swimming sideby-side for a long time. This attraction is associated with the long-range hydrodynamic interaction between the two droplets. The pusher swimmers induce a flow field that is directed toward the body, along their sides. Once two pushers swim on a converging course, they experience an attractive force [80] and reorient each other, until parallel side-by-side swimming is achieved [30]. It is also noteworthy that the particles never come in contact and the stable configuration is reached at a finite interparticle distance. This might be associated with the solute-mediated interactions where the two clouds of solutes around the droplets overlap, leading to a repulsive force [233]. Hence, the final distance may be determined by the interplay of the hydrodynamic attraction and the phoretic repulsion.

\subsection{Formation of spontaneously-rotating clusters}

The attractive hydrodynamic interactions discussed in the previous section can result in flow-induced self-assembly and phase separation in a crowded system $[79,80]$. To study the formation of such clusters, we filled a deep closed reservoir $(6 \mathrm{~mm}$ in diameter and $5 \mathrm{~mm}$ in depth) with an active emulsion of monodisperse droplets. Due to their higher mass density, the droplets gradually sediment to the bottom of the reservoir. 

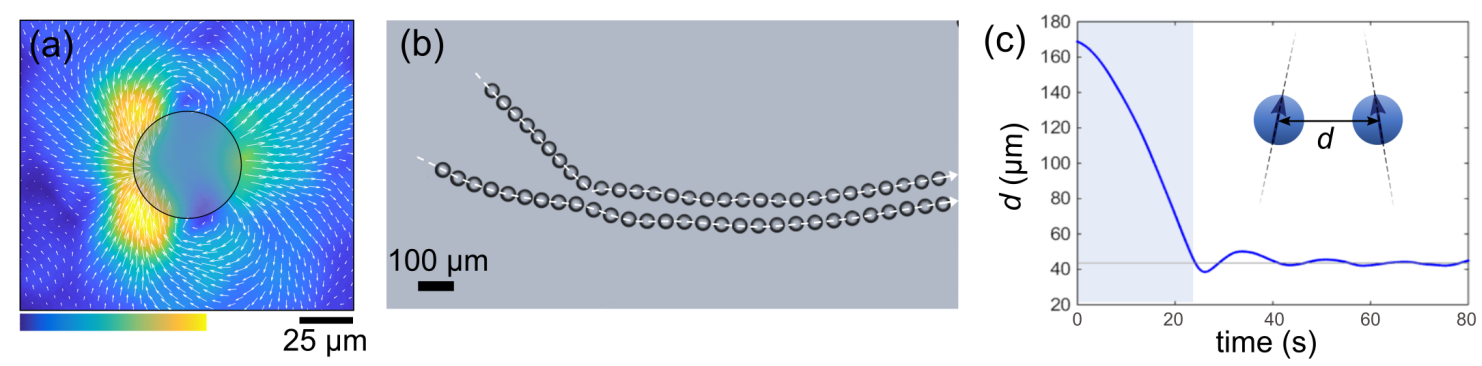

Figure 6.1 - (a) Mean flow field generated by a single freely swimming droplet inside a Hele-Shaw cell. The colormap shows the speed. (b) The interaction between two active droplets. We have overlaid the frames recorded during an $80 \mathrm{~s}$ period. In this particular data set, the droplets had shrunk somewhat due to the solubilization, before starting the recording. that is why their diameter is smaller than $50 \mu \mathrm{m}$. (c) Variation of the separation distance $d$ between the two droplets in time. The shaded region corresponds to the long-range hydrodynamic interactions leading to the formation of a droplet pair.

There, they start to interact with each other and autonomously assemble into planar clusters. This out-of-equilibrium process takes place in four stages (Fig. $6.2 \mathrm{a}-\mathrm{c}$ ): (i) Sedimentation, due to gravity, (ii) Aggregation, due to the mutual hydrodynamic attractive forces, (iii) Cluster formation, during which, the droplets form hexagonallyordered crystal-like structures and all droplet reorient to swim vertically against gravity, and (iv) Rising and Rotation, where the droplets cooperatively start to hover and rise, accompanied by a rotational motion. Since the system has no inherent broken symmetry - the droplets themselves are isotropic and rotation occurs even in symmetric heptamer structures like the one in Fig. $6.2 \mathrm{c}-$ the emergence of this rotational motion was unexpected and the symmetry needs to be broken by the nonlinear dynamics of the system. In this chapter we present experimental evidence of possible mechanisms.

We tracked seven individual droplets that eventually form a cluster. We have plotted the individual droplet trajectories centered around the coordinate of the middle droplet. As shown in Fig. 6.2 d, initially, droplets exhibit a random disordered motion which can be due to the chaotic propulsion dynamics (as discussed in Chapter 3) as well as multiple particle-particle and particle-boundary interactions. However, after the cluster is formed, the dynamics spontaneously change into an ordered state and steady rotational motion that is stable for a very long time.

All of the individual droplets in a cluster reorient to swim upwards against gravity, thereby generating a global convective flow which makes the cluster hover (Fig. 6.2 b) [79]. The planar arrangement of the cluster is stabilized by the interaction with the cell bottom [234]. Using the calibrated z-drive of the stage of an Olympus 
IX-83 microscope at $40 \times$ magnification, we measured the hovering height of clusters with different sizes. Fig. $6.2 \mathrm{e}$ demonstrates the variation of cluster height in time. Regardless of the cluster size (number of droplets $N$ ), they all tend to gradually rise with a speed on the order of $1 \mu \mathrm{m} / \mathrm{s}$. This continuous rise continues up to a height of several hundred $\mu \mathrm{m}$. Here, apparently, the stabilizing effect of the cell bottom has become so weak that the cluster disintegrates. We attribute the cluster's rise to the fact that the droplets dissolve and their mass decreases accordingly.

\subsection{Phase diagram for the cluster dynamics}

We now study the phase behavior of the clusters and characterize their dynamics. The two parameters that we choose to study are cluster size $N$ and Péclet number $(P e)$. We choose $N$ as a simple measure of collectivity in the cluster, while, as shown in Chapter 3, Pe characterizes the droplet dynamics at the individual scale.

For an active droplet, $P e$ is defined as [102]

$$
P e=\frac{V_{t} R_{d}}{D} \approx \frac{18 \pi^{2}}{k_{B} T} q_{s} r_{s}^{2} \zeta R_{d}^{2} \mu_{i}\left[\mu\left(\frac{2 \mu+3 \zeta / R_{d}}{2 \mu+3}\right)\right],
$$

where $V_{t}$ is the theoretical terminal droplet velocity in an external surfactant gradient $[43,54], R_{\mathrm{d}}=25 \mu \mathrm{m}$ the droplet radius, $D=\frac{k_{B} T}{6 \pi r_{s} \mu_{o}}$ the diffusion coefficient for the surfactant monomer (length scale $r_{s} \sim 10^{-10} \mathrm{~m}$ ), $q_{s}$ the isotropic interfacial surfactant consumption rate per area, $\zeta \sim 10 \mathrm{~nm}$ the characteristic length scale over which the surfactants interact with the droplet $[43,58], \mu_{o}$ and $\mu_{i}$ the viscosities of the outer and inner phases, and $\mu=\mu_{o} / \mu_{i}$. Pe represents the relative strength of the advective transport of surfactant molecules to their diffusion. It increases monotonically with droplet activity which here is proportional to $q_{s}$. We control this quantity by varying $c_{\text {TTAB }}$ - higher $c_{\text {TTAB }}$ results in higher $q_{s}$ (cf. Appendix 6.7).

In a space spanned by droplet activity $(P e)$ and cluster size $(N)$, we observe four regimes (Fig. 6.3a): (i) No clustering due to insufficient droplet activity, (ii) Non-rotating clusters, (iii) Rotating clusters, and (iv) unstable clusters due to high activity. At low $P e$, droplets generate weaker flow and only small clusters form $(N=3-5$ at $P e=5.2)$. By increasing $P e$, the droplet activity increases and in turn the hydrodynamic interactions are strong enough to form larger clusters. At $P e=6.8$ to 8.7 , the dynamics of smaller clusters $(N<8)$ transition from non-rotating to rotating. At sufficiently large $P e$, we observe unstable clusters that rearrange their 

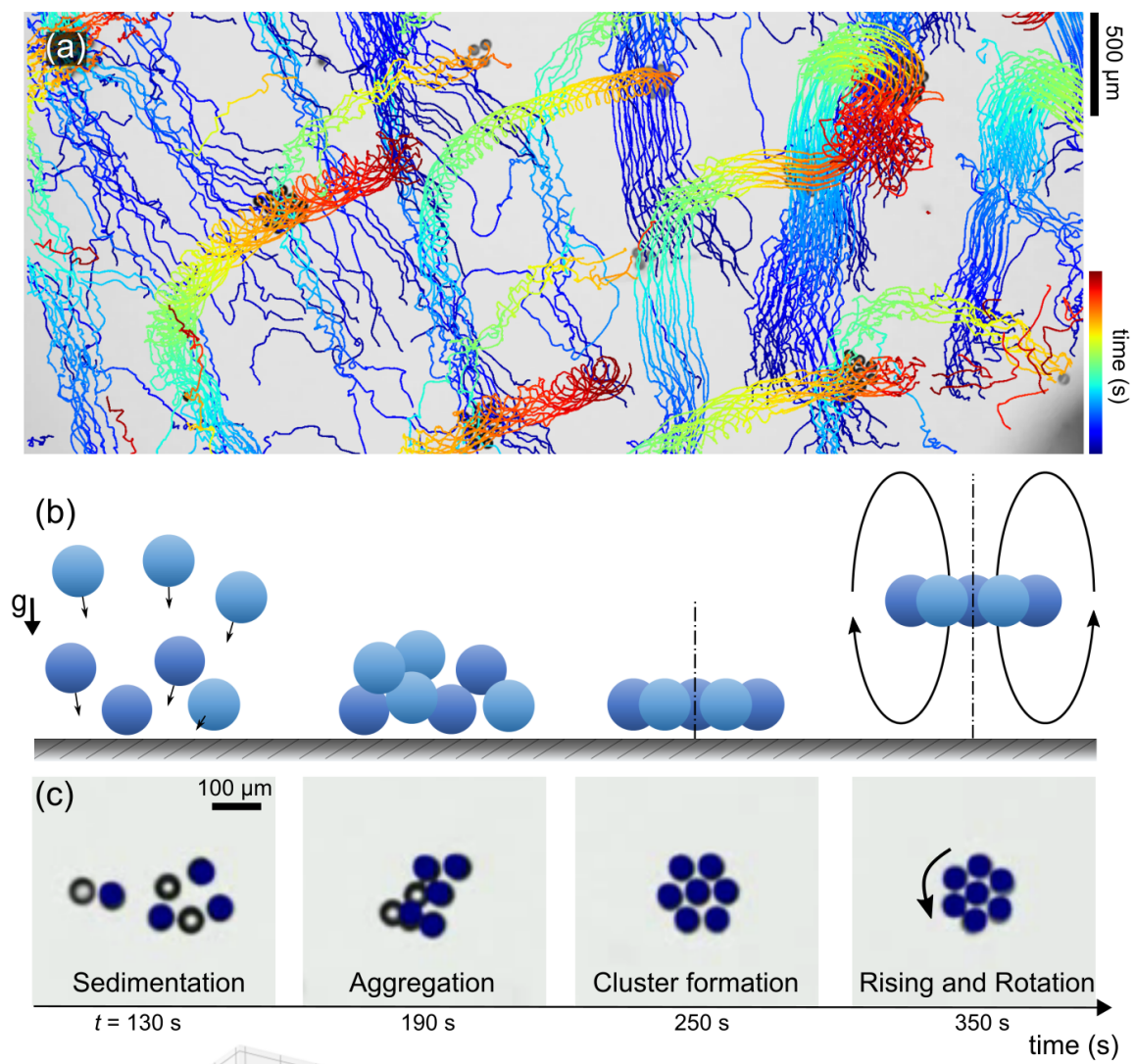

(d)

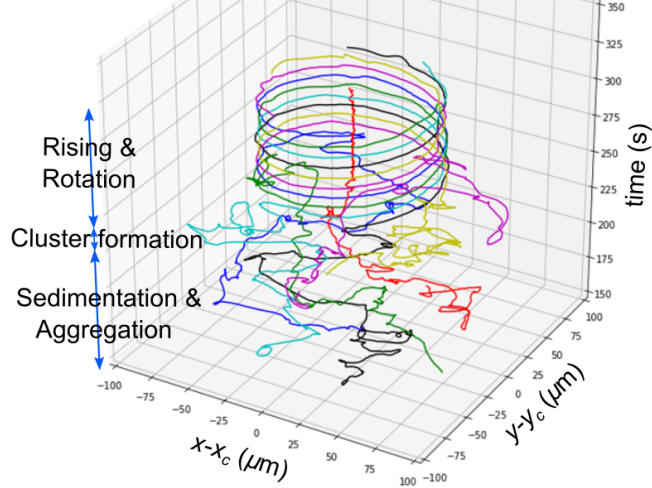

(e)

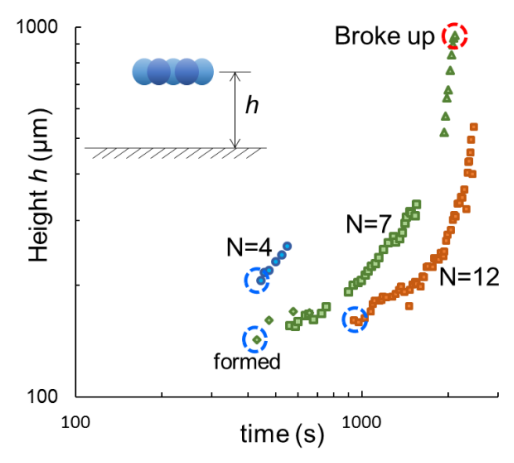

Figure 6.2 - (a) Trajectories of the individual droplets undergoing the four stages of flow-induced self-assembly resulting in spontaneously-rotating clusters. The micrograph is one snapshot from the experiment. Panel (b) schematically and panel (c) experimentally demonstrate the four different stages that a group of seven droplets undergo to form a rotating cluster. The colored droplets in (c) reside in the focal plane of the objective. (d) Trajectories of droplets forming one cluster. The z-axis represents the elapsed time. (e) Variation of cluster hovering height with time. 
structure frequently. In this regime, during the very short periods when the cluster possesses an ordered structure, it shows rotational dynamics before melting again into a disordered aggregate.

While increasing $P e$ results in the formation of larger clusters, the cluster size also influences the collective dynamics. As shown in Fig. 6.3 a, at a given $P e$, the cluster behavior changes from rotating to non-rotating with $N$. For instance, at $P e=10.9$, very large clusters of size $N>20$ barely rotate, while smaller clusters $(N<10)$ rotate steadily. This is reflected in Fig. $6.3 \mathrm{~b}$, in which we have plotted the measured rotational period of clusters with different $N$ at $P e=13.2$. The rotational velocity of a cluster linearly decreases with $N$. A similar trend was reported for Thiovulum majus bacteria that transition to downwards oriented bound states near surfaces [235] and self-organize into 2D clusters rotating on a solid boundary [232]. The clusters only rotate counter-clockwise since each individual, flagellated bacterium generates flow fields with counter-clockwise chirality. In contrast, droplet clusters, show both clockwise and counter-clockwise rotation and can spontaneously switch between the two. Hence, their rotation is not due to an internal chirality. We note, however, that chirality can be imposed on the cluster by using liquid crystalline droplets with a chiral dopant [79]. The significant dependence of the collective behavior on $P e$ suggests that the emergence of a rotating cluster can be attributed to the individual dynamics of the constituent droplets.

\subsection{Rotational broken symmetries in individual droplets}

To this end, we investigated helical states in the propulsion dynamics of individual droplets in response to variation of $P e$. By adding heavy water $\left(\mathrm{D}_{2} \mathrm{O}\right)$, we matched the density of the aqueous TTAB solution with that of the droplets. This allowed for observation of the individual droplet trajectories in a force-free, off-boundary bulk medium. In each experiment, we placed only a small number of droplets in the reservoir so that the inter-particle interactions are negligible. In Fig. 6.4, each image shows the overlaid trajectories of individual droplets at different $P e$. At low $P e$, the droplets show a persistent and directed motion. At intermediate values $(P e=6.8$ to 8.7 ), the symmetry is spontaneously broken, and the droplets swim in a helical trajectory with no global handedness. At $P e \geq 10.9$, droplets demonstrate increasingly chaotic swimming behavior. In our previous experiments in quasi-2D confinement (Chapter 3), we demonstrated that, due to the non-linearity of the surfactant advection, 

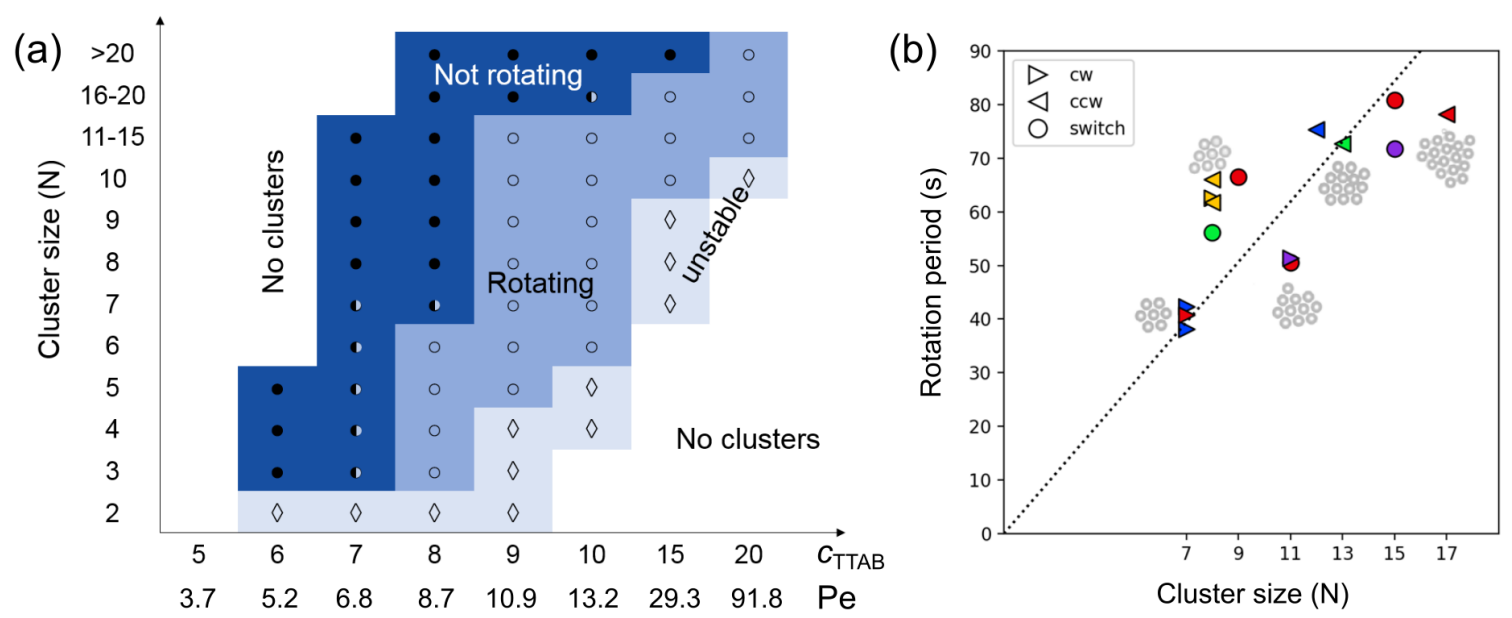

Figure 6.3 - (a) The phase diagram of the cluster dynamics and stability. In the horizontal axis, the $c_{\mathrm{TTAB}}$ values and the corresponding $P e$ values are presented. and $\circ$ denote non-rotating and rotating clusters, respectively. The half-full circle corresponds to the margin where both behaviors are observed. $\diamond$ corresponds to unstable clusters. (b) Rotational period of the clusters versus their size, labels denote clockwise, counterclockwise and switching rotation.

by increasing $P e$ the propulsion dynamics transition from steady and persistent to unsteady and chaotic. Now, in 3D, remarkably, there appears to be a far more ordered and steady helical swimming regime between straight and chaotic propulsion. While a full understanding of this intermediate helical regime appears unfeasible without extensive numerical modelling, we can at least speculate about possible origins based on our previous findings. As shown in Chapter 3, for intermediate $P e$ values in 2D, higher hydrodynamic modes are excited momentarily, generating a flow with quadrupolar symmetry. Such flow results in a accumulation of filled micelles ("spent fuel") at the front stagnation point of the droplet. This accumulation results in a torque imbalance, reorienting the droplet away from this self-made chemical trap. The droplet undergoes recurrent reorientations in a semi-periodic manner resulting in a quasi-meandering trajectory (Fig. $3.3 \mathrm{~b}$ and Fig. $3.5 \mathrm{~b}$ ). While the dynamics in a Hele-Shaw cell are confined to two dimensions, in a $3 \mathrm{D}$ bulk medium the droplet can now reorient within the entire angular space, allowing for more complex modes of symmetry breaking which can lead to helical states $[54,102,116]$. A similar dynamical instability is a possible mechanism causing the rotational motion of planar clusters. In future work, we will investigate these instabilities using our established methods of imaging chemical and flow fields around a rotating cluster (Chapter 3). 


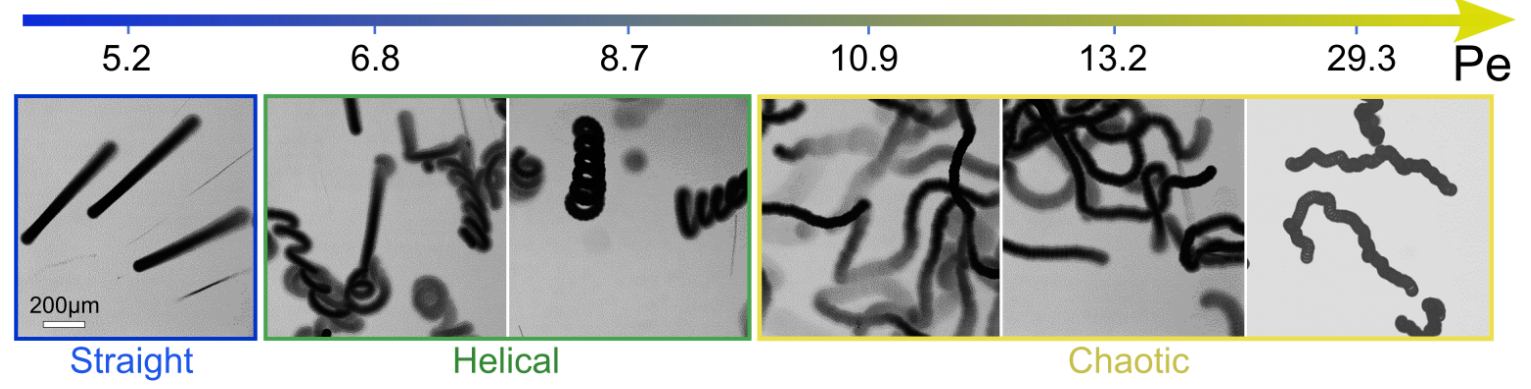

Figure 6.4 - Dynamical behavior of individual active droplets with varying $P e$. The Pe range for each regime is shown by a different color.

\subsection{Discussion and Conclusion}

We demonstrated that the dynamics of isotropic droplet swimmers transition from straight to helical and then to chaotic when the interfacial activity is increased (characterized via $P e$ ). This dynamical instability, which is solely due to the nonlinearity of surfactant transport, could explain the emergence of rotating clusters. However, this intermediate regime is limited to a very narrow range of $6.8 \leq P e \leq 8.7$. Beyond $P e=8.7$, the dynamics become increasingly chaotic. In contrast, the planar clusters exhibit rotational dynamics within the range of $6.8 \leq P e \leq 91.8$ which is remarkably broader compared to the range for the individual droplets. In other words, the clusters show ordered rotational motion, even at a $P e$ range corresponding to the chaotic motion of its constituent elements. This suggests that the cluster formation suppresses the chaotic dynamics of the individual droplets and integrates them into an ordered collective system which performs a synchronized rotational motion. This stability is achieved cooperatively through mutual hydrodynamic interactions between the constituent elements of the clusters. At very high $P e$, only very large clusters maintain their ordered structure (stability) and undergo rotation. While the rotational motion of the clusters is reminiscent of the collective dynamics in the suspensions of Thiovulum majus bacterium, it is essentially different since for the bacteria, the rotational flow arises from their chiral body shape, whereas for the isotropic droplets, the symmetry is spontaneously broken due to the nonlinearity of the surfactant advection.

In conclusion, we demonstrated that a simple emulsion of active droplets can use hydrodynamic interactions to self-organize into rotating planar clusters reminiscent of that in living systems. We attributed the emergence of this rotational dynamics to the complex coupling between the hydrodynamics and the chemical transport. We characterized the 3D dynamics of individual droplets based on $P e$ and identified three 
regimes of persistent, helical, and chaotic swimming. We observed that the cluster formation suppresses the chaotic fluctuations of individual droplets at high $P e$, resulting in the emergence of ordered rotating clusters of isotropic droplets. We showed that the collective behavior, in terms of rotation and stability, can be controlled by tuning the activity $(P e)$ and the cluster size.

\subsection{Appendix}

\section{Dissolution rate of active droplets}

To estimate the surfactant consumption rate $q_{s}$ to calculate $P e$, we measured the droplet shrinking rate $\mathrm{d} R_{d} / \mathrm{d} t$ (plotted in Fig. 6.5). We found a dependence on the $c_{\text {TTAB }}$. We used a first order approximation, via linear regression (black line), for $\mathrm{d} R_{d} / \mathrm{d} t$, to calculate $q_{s}$ for different $c_{\mathrm{TTAB}}$ values. $q_{s}$ is estimated by relating the dissolution rate of the active droplet to the isotropic surfactant consumption at the droplet interface [58]

\section{Viscosity measurements}

We measured the viscosity of the oil phase $\mu_{\mathrm{i}}=138 \mathrm{mPa} . \mathrm{s}$ and the surfactant solution. The measured values were $\mu_{\mathrm{i}}=1.3,1.6,1.9$, and $2.9 \mathrm{mPa} . \mathrm{s}$, corresponding to $c_{\mathrm{TTAB}}=$ 5, 10, 15, and $20 \mathrm{wt} . \%$, respectively. The estimated values, corresponding to other $c_{\mathrm{TTAB}}$, were extracted by interpolation.

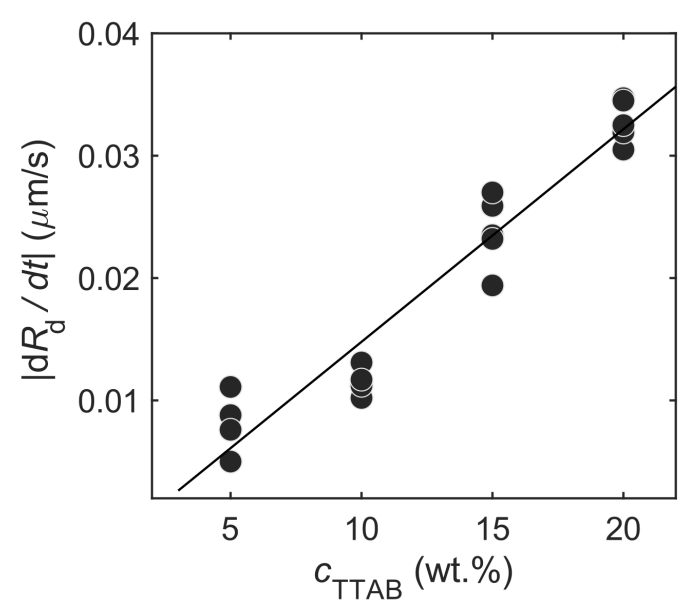

Figure 6.5 - Dissolution rate of active droplets $\mathrm{d} R_{d} / \mathrm{d} t$ versus different $c_{\text {TTAB }}$ values. 


\section{Chapter 7}

\section{Discussion and Outlook}

Non-equilibrium physics has paved the way for a new, exponentially growing research field known as active soft matter. It has provided us great insight into our efforts to understand the physics of living matter. The very appealing possibility, offered by active soft matter, is the promise of developing an artificial, physical system that mimics living matter. The construction of such biomimetic systems from soft components has two main benefits. From a fundamental perspective, it can provide ground to test the hypotheses about the underlying physics behind the biological functions. From a technological point of view, it offers the possibility of employing the same principles to create synthetic functional materials that possess some type of autonomy and can perform tasks that are currently beyond the state of the art.

The main objectives of this thesis were to explore the biomimetic features of active droplets and study the emergent collective behavior in active emulsions. In Chapter 1, we discussed the hallmarks of living systems and the challenges in developing a life-like protocell, in the framework of active droplets.

In Chapter 2, we addressed the challenge of compartmentalization. We used microfluidics to produce composite droplets known as double emulsions-where a water droplet is placed inside an oil droplet. Motility in such a design results in coalescence and the liquid shell breaks up. We addressed this challenge using a nematic liquid crystal as the shell. The nematoelasticity of the liquid crystal balances the hydrodynamic drag force on the water droplet and impede the coalescence. We studied the peculiar propulsion dynamics of the active shells in $2 \mathrm{D}$ and $3 \mathrm{D}$ and provided routes to control the motion via topology, chemical signaling and hydrodynamic interactions with the boundaries.

In chapter 3, we studied the adaptability of an active droplet to variation of 
viscosity in the outer environment. We developed a novel technique based on dualchannel fluorescent microscopy to visualize the hydrodynamics and chemical fields simultaneously and investigate the spatiotemporal dynamics of the system. We observed the emergence of chaotic propulsion with increasing the viscosity of the swimming medium. Using the visualization technique, we demonstrated the bimodal nature of this chaotic motion. Our results show that the slowly-decaying products of activity have a memory effect on the dynamics of the droplets. The recurrent switching between flow modes and the self-avoiding random walks are both caused by interactions of the droplet with the chemical products-a critical factor that has been overlooked so far.

Chapters 4 and 5 focus on the information processing aspect of the active droplets. In Chapter 4, we studied the dynamics, governed by the interplay of topography (pillars of different diameter) and negative autochemotaxis. We reported on how the curvature of the boundary and autochemotaxis can lead to different scenarios: no wall-attachment for highly curved interfaces, stable trapping at large pillars, and a narrow transition region where interaction with own chemical trail makes the droplet detach after a single orbit. In Chapter 5, we used fluorescent imaging of the filled micelle trail to measure their diffusion coefficient. We quantified the autochemotactic interactions between droplets and the trails of their counterparts and obtained a phase diagram based on delay time and incident angle. Next, we studied the collective dynamics, governed by autochemotactic interactions, in active emulsions. We report a novel dynamical arrest mechanism: transient autochemotactic caging where a droplet is trapped inside a cage made of the trails of other droplets. By extending the study to 3D, we showed caging in unconfined suspensions of active droplets.

A significant appeal of active particles is their ability to self-organize and exhibit phase separation. Artificial prototypes of active particles enabled us to study the underlying physics of such collective behavior in the absence of any biological complexity. In Chapter 6, we studied active emulsions in which droplets undergo flow-induced phase separation in the vicinity of a boundary $[79,80]$. We report spontaneous rotation in clusters of isotropic active droplets. We trace this symmetry breaking to the dynamics of the individual swimmers. At higher interfacial activity rates, the nonlinear coupling between the hydrodynamics and the chemical transport results in spontaneous helical swimming for a droplet. The helical swimming only happens in a very narrow transition region, before the droplet switches to chaotic motion. In a cluster, however, collective hydrodynamic interactions suppress the chaotic dynamics, changing it into an ordered rotational motion of a cluster. 
In conclusion, we explored active droplets as a paradigm for biomimetic synthetic microswimmers. They are driven by chemical activity and generate long-lived chemical gradients in the environment. Unlike most artificial microswimmers with built-in polarity, active droplets are radially isotropic. Thus, they can undergo multiple spontaneous symmetry breaking scenarios depending on the environmental cues. These characteristics make active droplets an elegant, versatile and responsive model system. They are indeed an experimental realization for the squirmer model introduced by Lighthill in 1952 [236] and thus can be used to validate the theoretical predictions in fundamental fluid dynamics.

To acquire insight into the emergence of collective behavior and phase transitions in out-of-equilibrium systems, active droplets can be used to understand pair interactions and build our knowledge from the bottom up to more complicated many-body systems. The framework and the techniques that we introduced advance the state of the art of the field enabling further quantitative studies of the complex dynamics, propulsion strategies, or more sophisticated composite designs.

During the course of this study, the spectrum of the research ideas broadened and new possibilities and challenges emerged.

To continue the work presented in Chapter 5, we are currently collaborating with Prof. Marco Mazza, from Loughborough University, and Dr. Fabian Schwarzendahl, from the University of California in Merced, on the theoretical modeling of the collective dynamics governed by autochemotactic interactions (caging in a two-dimensional system).

To demonstrate the generality of our framework, we collaborated with a chemistry group (Prof. David Officer, University of Wollongong, Australia) who developed pHresponsive active droplets. The Marangoni stress that propels the droplet is caused by a deprotonation reaction in an external alkaline gradient. The deprotonation of merocyanine salt $\mathrm{MCH}+\mathrm{DBS}-$ by base $(\mathrm{NaOH})$ liberates the surfactant sodium dodecylbenzene sulphonate (NaDBS) and give the free merocyanine MC. The MC molecules possess a red color. As an active droplet propels, it secrets MC molecules in its wake- similar to the secretion of filled micelles by a solubilizing droplet- in the form of one or two visible red plumes, depending on the propulsive mode. We used the same approach as introduced in Chapter 3, to identify the propulsive mode of the droplets, and studied how active droplets adapt their dynamics in response to a chemical gradient. We observed the suppression of higher modes of interfacial activity due to $\mathrm{pH}$ gradients resulting in a transition from chaotic to straight motion at stronger gradients. We are 
further collaborating on developing light-activated swimming droplets.

While the majority of studies, including the work in Chapter 3, assume a constant reaction rate at the interface, the validity of this assumption has not been proven yet. We are currently studying the effect of the local concentration of products of activity on the reaction rate at the interface. We have performed high-resolution flow measurement and chemical field visualization to tackle this problem. We have observed that the presence of filled micelles (spent fuel) inhibits the reaction at the interface and weakens the Marangoni stress.

Future research on the individual dynamics of active droplets should focus on the memory effects due to the presence of slowly-decaying products of activity. Understanding such effects can explain the emergence of helical swimming for isotropic droplets and provide us further insights on how to tune the physicochemical properties of the system to achieve more complex functionalities. The application of the joint visualization technique, introduced in this thesis, can be very informative if combined with direct numerical simulations of the system in three dimensions. Such simulations can facilitate the systematic study of the dynamics by producing phase diagrams based on many numerical experiments.

To continue the collective dynamics in active emulsions, now that we have the capability to perform time-resolved joint visualization of the involved fields, the starting point should be pair interactions. There are multiple theoretical studies [163-166] that can be used for comparison. A fascinating phenomenon in bacterial colonies is their wave-like collective migration due to a self-generated chemical gradient [211]. This phenomenon can also be observed in active emulsions since they leave behind chemo-repellents, generating a gradient that might cause a collective migration to the unexplored regions. Such behavior would be even more interesting to study in model porous media, such as artificial soil, where the swimming mechanism is influenced by the hydrodynamic interactions with the boundaries. Such measurements are now possible, even in $3 \mathrm{D}$, via refractive-index matching of the the bulk swimming medium. 


\section{References}

[1] K. Tunstrøm, Y. Katz, C. C. Ioannou, C. Huepe, M. J. Lutz, and I. D. Couzin. "Collective states, multistability and transitional behavior in schooling fish". In: PLoS Comput Biol 9.2 (2013), e1002915.

[2] A. Cavagna, D. Conti, C. Creato, L. Del Castello, I. Giardina, T. S. Grigera, S. Melillo, L. Parisi, and M. Viale. "Dynamic Scaling in Natural Swarms". In: Nature Physics 13.9 (2017), pp. 914-918.

[3] H. Chaté and M. A. Muñoz. "Insect Swarms Go Critical". In: Physics 7 (2014).

[4] J. Dunkel, S. Heidenreich, K. Drescher, H. H. Wensink, M. Bär, and R. E. Goldstein. "Fluid dynamics of bacterial turbulence." In: Physical review letters 110.22 (May 2013), p. 228102. DOI: 10.1103/PhysRevLett.110.228102.

[5] N. Bain and D. Bartolo. "Dynamic response and hydrodynamics of polarized crowds". In: Science 363.6422 (2019), pp. 46-49.

[6] V. Schaller, C. Weber, C. Semmrich, E. Frey, and A. R. Bausch. "Polar patterns of driven filaments". In: Nature 467.7311 (2010), pp. 73-77.

[7] Y. Sumino, K. H. Nagai, Y. Shitaka, D. Tanaka, K. Yoshikawa, H. Chaté, and K. Oiwa. "Large-scale vortex lattice emerging from collectively moving microtubules". In: Nature 483.7390 (2012), pp. 448-452.

[8] M. Ballerini, N. Cabibbo, R. Candelier, A. Cavagna, E. Cisbani, I. Giardina, V. Lecomte, A. Orlandi, G. Parisi, A. Procaccini, et al. "Interaction ruling animal collective behavior depends on topological rather than metric distance: Evidence from a field study". In: Proceedings of the national academy of sciences 105.4 (2008), pp. 1232-1237.

[9] M. E. Cates and J. Tailleur. "Motility-Induced Phase Separation". In: Annual Review of Condensed Matter Physics 6.1 (2015), pp. 219-244.

[10] A. Varma. "Active fluids-Interactions and collective dynamics in phoretic suspensions". PhD thesis. Université Paris-Saclay, 2019.

[11] J. Costerton, L. Montanaro, and C. R. Arciola. "Biofilm in implant infections: its production and regulation". In: The International journal of artificial organs 28.11 (2005), pp. 1062-1068.

[12] H.-C. Flemming. "Biofouling in water systems-cases, causes and countermeasures". In: Applied microbiology and biotechnology 59.6 (2002), pp. 629-640.

[13] S. Herminghaus, C. C. Maass, C. Krüger, S. Thutupalli, L. Goehring, and C. Bahr. "Interfacial Mechanisms in Active Emulsions". In: Soft Matter 10.36 (2014), pp. 7008-7022. 
[14] A. Sokolov, M. M. Apodaca, B. A. Grzybowski, and I. S. Aranson. "Swimming bacteria power microscopic gears". In: Proceedings of the National Academy of Sciences 107.3 (2010), pp. 969-974.

[15] A. Sokolov and I. S. Aranson. "Reduction of viscosity in suspension of swimming bacteria". In: Physical Review Letters 103.14 (2009), p. 148101.

[16] H. M. López, J. Gachelin, C. Douarche, H. Auradou, and E. Clément. "Turning bacteria suspensions into superfluids". In: Physical review letters 115.2 (2015), p. 028301.

[17] M. B. Miller and B. L. Bassler. "Quorum sensing in bacteria". In: Annual Reviews in Microbiology 55.1 (2001), pp. 165-199.

[18] N. A. Yewdall, A. F. Mason, and J. C. Van Hest. "The hallmarks of living systems: towards creating artificial cells". In: Interface Focus 8.5 (2018), p. 20180023.

[19] C. de Blois. "Swimming water droplets in complex environments, Confinement, gravity and collective effects." PhD thesis. PSL university, 2019.

[20] P. Bateson. "Adaptability and Evolution". In: Interface Focus 7.5 (2017), p. 20160126.

[21] G. Jékely, J. Colombelli, H. Hausen, K. Guy, E. Stelzer, F. Nédélec, and D. Arendt. "Mechanism of phototaxis in marine zooplankton". In: Nature 456.7220 (Nov. 2008), pp. 395-399. DOI: 10.1038/nature07590.

[22] C. T. T. Lefèvre, M. Bennet, L. Landau, P. J. Vach, D. Pignol, D. A. A. Bazylinski, R. B. B. Frankel, S. Klumpp, and D. Faivre. "Diversity of MagnetoAerotactic Behaviors and Oxygen Sensing Mechanisms in Cultured Magnetotactic Bacteria". In: Biophysical Journal 107.2 (July 2014), pp. 527-538. DOI: 10.1016/j.bpj.2014.05.043.

[23] J. A. Wondergem, M. Mytiliniou, F. C. de Wit, T. G. Reuvers, D. Holcman, and D. Heinrich. "Chemotaxis and topotaxis add vectorially for amoeboid cell migration". In: bioRxiv (2019), p. 735779.

[24] S. Klumpp and D. Faivre. "Magnetotactic bacteria". In: The European Physical Journal Special Topics 225.11-12 (2016), pp. 2173-2188.

[25] A. Roberts. "Mechanisms of gravitaxis in Chlamydomonas". In: The Biological Bulletin 210.2 (2006), pp. 78-80.

[26] A. J. T. M. Mathijssen, N. Figueroa-Morales, G. Junot, É. Clément, A. Lindner, and A. Zöttl. "Oscillatory Surface Rheotaxis of Swimming E. Coli Bacteria". In: Nature Communications 10.1 (2019), p. 3434.

[27] H. C. Fu, T. R. Powers, R. Stocker, et al. "Bacterial rheotaxis". In: Proceedings of the National Academy of Sciences 109.13 (2012), pp. 4780-4785.

[28] S. Ramaswamy. "The mechanics and statistics of active matter". In: (2010).

[29] D. Needleman and Z. Dogic. "Active matter at the interface between materials science and cell biology". In: Nature Reviews Materials 2.9 (2017), pp. 1-14.

[30] E. Lauga and T. R. Powers. "The Hydrodynamics of Swimming Microorganisms". In: Reports on Progress in Physics 72.9 (2009), p. 096601. 
[31] E. M. Purcell. "Life at low Reynolds number". In: American journal of physics 45.1 (1977), pp. 3-11.

[32] F. J. Schwarzendahl. "Collective Microswimmer Motility in Complex Environments." Dissertation. Georg-August-Universität Göttingen, 2018.

[33] M. T. Downton and H. Stark. "Simulation of a model microswimmer". In: Journal of Physics: Condensed Matter 21.20 (2009), p. 204101.

[34] K. Drescher, J. Dunkel, L. H. Cisneros, S. Ganguly, and R. E. Goldstein. "Fluid dynamics and noise in bacterial cell-cell and cell-surface scattering". In: Proceedings of the National Academy of Sciences 108.27 (2011), pp. 10940-10945.

[35] K. Drescher, R. E. Goldstein, N. Michel, M. Polin, and I. Tuval. "Direct measurement of the flow field around swimming microorganisms". In: arXiv (2010), pp. 19-22. DOI: 10.1103/PhysRevLett.105.168101.

[36] C. Bechinger, R. Di Leonardo, H. Löwen, C. Reichhardt, G. Volpe, and G. Volpe. "Active particles in complex and crowded environments". In: Reviews of Modern Physics 88.4 (2016), p. 045006.

[37] M. Driscoll, B. Delmotte, M. Youssef, S. Sacanna, A. Donev, and P. Chaikin. "Unstable fronts and motile structures formed by microrollers". In: Nature Physics 13.4 (2017), pp. 375-379.

[38] A. Kaiser, A. Snezhko, and I. S. Aranson. "Flocking ferromagnetic colloids". In: Science advances 3.2 (2017), e1601469.

[39] J. L. Moran and J. D. Posner. "Phoretic self-propulsion". In: Annual Review of Fluid Mechanics 49 (2017), pp. 511-540.

[40] P. Illien, R. Golestanian, and A. Sen. "Fuelled'motion: Phoretic motility and collective behaviour of active colloids". In: Chemical Society Reviews 46.18 (2017), pp. 5508-5518.

[41] J. R. Howse, R. A. Jones, A. J. Ryan, T. Gough, R. Vafabakhsh, and R. Golestanian. "Self-Motile Colloidal Particles: From Directed Propulsion to Random Walk". In: Physical review letters 99.4 (2007), p. 048102.

[42] R. Dreyfus, J. Baudry, M. L. Roper, M. Fermigier, H. a. Stone, and J. Bibette. "Microscopic artificial swimmers". In: Nature 437.7060 (Oct. 2005), pp. 862-865. DOI: $10.1038 /$ nature04090.

[43] J. L. Anderson. "Colloid Transport by Interfacial Forces". In: Annual Review of Fluid Mechanics 21.1969 (1989), pp. 61-99.

[44] N. Young, J. S. Goldstein, and M. J. Block. "The motion of bubbles in a vertical temperature gradient". In: Journal of Fluid Mechanics 6.3 (1959), pp. 350-356.

[45] M. M.-J. Lin and D. C. Prieve. "Electromigration of latex induced by a salt gradient". In: Journal of colloid and interface science 95.2 (1983), pp. 327-339.

[46] W. J. Lechnick and J. A. Shaeiwitz. "Measurement of diffusiophoresis in liquids". In: Journal of colloid and interface science 102.1 (1984), pp. 71-87.

[47] J. Ebel, J. L. Anderson, and D. Prieve. "Diffusiophoresis of latex particles in electrolyte gradients". In: Langmuir 4.2 (1988), pp. 396-406. 
[48] P. O. Staffeld and J. A. Quinn. "Diffusion-induced banding of colloid particles via diffusiophoresis: 2. Non-electrolytes". In: Journal of colloid and interface science 130.1 (1989), pp. 88-100.

[49] P. O. Staffeld and J. A. Quinn. "Diffusion-induced banding of colloid particles via diffusiophoresis: 1. Electrolytes". In: Journal of colloid and interface science 130.1 (1989), pp. 69-87.

[50] B. Derjaguin, G. Sidorenkov, E. Zubashchenkov, and E. Kiseleva. "Kinetic phenomena in boundary films of liquids". In: Kolloidn. zh 9.01 (1947).

[51] C. Marangoni. "Sull'espansione delle goccie d'un liquido galleggianti sulla superficie di altro liquido (On the expansion of a droplet of a liquid floating on the surface of another liquid)(Pavia, Italy: fratelli Fusi (Fusi brothers)". In: Pavia, Italy (1869).

[52] J. Thomson. "XLII. On certain curious motions observable at the surfaces of wine and other alcoholic liquors". In: The London, Edinburgh, and Dublin Philosophical Magazine and Journal of Science 10.67 (1855), pp. 330-333.

[53] C. De Blois de La Calande. "Goutte d'eau Nageuse En Environnement Complexe, Confinement, Gravité et Effets Collectifs." These de Doctorat. Paris Sciences et Lettres, 2019.

[54] M. Morozov and S. Michelin. "Nonlinear Dynamics of a Chemically-Active Drop: From Steady to Chaotic Self-Propulsion". In: The Journal of Chemical Physics 150.4 (2019), p. 044110.

[55] R. Golestanian, T. Liverpool, and A. Ajdari. "Designing phoretic micro-and nano-swimmers". In: New Journal of Physics 9.5 (2007), p. 126.

[56] S. Michelin and E. Lauga. "Autophoretic locomotion from geometric asymmetry". In: The European Physical Journal E 38.2 (2015), p. 7.

[57] S. Michelin, E. Lauga, and D. Bartolo. "Spontaneous Autophoretic Motion of Isotropic Particles". In: Physics of Fluids 25.6 (2013), p. 061701.

[58] Z. Izri, M. N. van der Linden, S. Michelin, and O. Dauchot. "Self-Propulsion of Pure Water Droplets by Spontaneous Marangoni-Stress-Driven Motion". In: Physical Review Letters 113.24 (2014), p. 248302.

[59] T. Toyota, H. Tsuha, K. Yamada, K. Takakura, T. Ikegami, and T. Sugawara. "Listeria-like motion of oil droplets". In: Chemistry letters 35.7 (2006), pp. 708709.

[60] S. Thutupalli and S. Herminghaus. "Tuning Active Emulsion Dynamics via Surfactants and Topology". In: European Physical Journal E 36.8 (2013), p. 91.

[61] H. Kitahata, N. Yoshinaga, K. H. Nagai, and Y. Sumino. "Spontaneous Motion of a Droplet Coupled with a Chemical Wave". In: Phys. Rev. E 84.1 (2011), p. 015101.

[62] H. Kitahata, R. Aihara, N. Magome, and K. Yoshikawa. "Convective and Periodic Motion Driven by a Chemical Wave". In: J. Chem. Phys. 116.13 (2002), pp. 5666-5672. 
[63] M. M. Hanczyc, T. Toyota, T. Ikegami, N. Packard, and T. Sugawara. "Fatty Acid Chemistry at the Oil-Water Interface: Self-Propelled Oil Droplets". In: J. Am. Chem. Soc. 129.30 (2007), pp. 9386-9391.

[64] T. Ban, T. Yamagami, H. Nakata, and Y. Okano. "pH-Dependent Motion of Self-Propelled Droplets due to Marangoni Effect at Neutral pH". In: Langmuir 29.8 (Feb. 2013), pp. 2554-2561. DOI: 10.1021/la3047164.

[65] S. Thutupalli, R. Seemann, and S. Herminghaus. "Swarming Behavior of Simple Model Squirmers". In: New J. Phys. 13.7 (2011), p. 073021.

[66] T. Toyota, H. Tsuha, K. Yamada, K. Takakura, T. Ikegami, and T. Sugawara. "Listeria-like Motion of Oil Droplets". In: Chemistry Letters 35.7 (2006), pp. 708 709. DOI: $10.1246 / \mathrm{cl} .2006 .708$.

[67] C. C. Maass, C. Krüger, S. Herminghaus, and C. Bahr. "Swimming Droplets". In: Annual Review of Condensed Matter Physics 7.1 (2016), pp. 171-193.

[68] M. Li, M. Hosseinzadeh, I. Pagonabarraga, R. Seemann, M. Brinkmann, and J.-B. Fleury. "Kinetics of Active Water/Ethanol Janus Droplets". In: Soft Matter 16.29 (2020), pp. 6803-6811.

[69] T. Ban, T. Yamada, A. Aoyama, Y. Takagi, and Y. Okano. "CompositionDependent Shape Changes of Self-Propelled Droplets in a Phase-Separating System". In: Soft Matter 8.14 (2012), pp. 3908-3916.

[70] T. Ban, T. Fukuyama, S. Makino, E. Nawa, and Y. Nagatsu. "Self-Propelled Vesicles Induced by the Mixing of Two Polymeric Aqueous Solutions through a Vesicle Membrane Far from Equilibrium". In: Langmuir 32.11 (2016), pp. 25742581.

[71] A. Peña and C. A. Miller. "Solubilization rates of oils in surfactant solutions and their relationship to mass transport in emulsions". In: Advances in colloid and interface science 123.0 (2006), pp. 241-257. DOI: 10.1016/j.cis.2006.05.005.

[72] S. Ariyaprakai and S. R. Dungan. "Contribution of molecular pathways in the Micellar solubilization of monodisperse emulsion droplets". In: Langmuir 24.7 (Apr. 2008), pp. 3061-3069. DOI: 10.1021/la703204c.

[73] M. Morozov and S. Michelin. "Self-Propulsion near the Onset of Marangoni Instability of Deformable Active Droplets". In: Journal of Fluid Mechanics 860 (2019), pp. 711-738. arXiv: 1810.03983.

[74] J. Meixner, S. de Groot, and P. Mazur. Non-Equilibrium Thermodynamics. 1962.

[75] J. Kennedy. "Swarm intelligence". In: Handbook of nature-inspired and innovative computing. Springer, 2006, pp. 187-219.

[76] I. Buttinoni, J. Bialké, F. Kümmel, H. Löwen, C. Bechinger, and T. Speck. "Dynamical clustering and phase separation in suspensions of self-propelled colloidal particles". In: Physical review letters 110.23 (2013), p. 238301.

[77] R. Singh and R. Adhikari. "Generalized Stokes laws for active colloids and their applications". In: Journal of Physics Communications 2.2 (2018), p. 025025. 
[78] J. Palacci, S. Sacanna, A. P. Steinberg, D. J. Pine, and P. M. Chaikin. "Living crystals of light-activated colloidal surfers". In: Science 339.6122 (2013), pp. 936940.

[79] C. Krueger, C. Bahr, S. Herminghaus, and C. C. Maass. "Dimensionality matters in the collective behaviour of active emulsions". In: The European Physical Journal E 39.6 (2016), p. 64.

[80] S. Thutupalli, D. Geyer, R. Singh, R. Adhikari, and H. A. Stone. "Flow-induced phase separation of active particles is controlled by boundary conditions". In: Proceedings of the National Academy of Sciences 115.21 (2018), pp. 5403-5408.

[81] A. Varma, T. D. Montenegro-Johnson, and S. Michelin. "Clustering-Induced Self-Propulsion of Isotropic Autophoretic Particles". In: Soft Matter 14.35 (2018), pp. $7155-7173$.

[82] A. Kamphuis. "Picture distributed under a cc by-sa 4.0 licence, bioconvection patterns of euglena gracilis in 5 petri dishes of different height and diameter, 2 time steps". In: https://de.wikipedia.org/wiki/Biokonvektion\#/media/File: Bioconvection_Euglena_5_Petri_Dishes_2_times_Colour.jpg (2015).

[83] X. Bou. "Interview with Xavi Bou". In: www.odhotels.com/de/magazine/interview-with-xavi-bou/ (also see http://xavibou.com/).

[84] B. Liebchen, D. Marenduzzo, I. Pagonabarraga, and M. E. Cates. "Clustering and Pattern Formation in Chemorepulsive Active Colloids". In: Physical Review Letters 115.25 (2015), p. 258301.

[85] E. Lushi, R. E. Goldstein, and M. J. Shelley. "Collective Chemotactic Dynamics in the Presence of Self-Generated Fluid Flows". In: Physical Review E 86.4 (2012), p. 040902.

[86] I. Theurkauff, C. Cottin-Bizonne, J. Palacci, C. Ybert, and L. Bocquet. "Dynamic Clustering in Active Colloidal Suspensions with Chemical Signaling". In: Physical Review Letters 108.26 (2012), p. 268303.

[87] O. Pohl and H. Stark. "Dynamic Clustering and Chemotactic Collapse of SelfPhoretic Active Particles". In: Physical Review Letters 112.23 (2014), p. 238303.

[88] T. Bickel, G. Zecua, and A. Würger. "Polarization of active Janus particles". In: Physical Review E 89.5 (May 2014), p. 050303. DOI: 10.1103/PhysRevE.89. 050303.

[89] C. Jin, C. Krüger, and C. C. Maass. "Chemotaxis and Autochemotaxis of Self-Propelling Droplet Swimmers". In: Proceedings of the National Academy of Sciences 114.20 (2017), pp. 5089-5094.

[90] W. T. Kranz, A. Gelimson, K. Zhao, G. C. L. Wong, and R. Golestanian. "Effective Dynamics of Microorganisms That Interact with Their Own Trail". In: Phys. Rev. Lett. 117 (3 2016), p. 038101.

[91] A. Gelimson, K. Zhao, C. K. Lee, W. T. Kranz, G. C. Wong, and R. Golestanian. "Multicellular self-organization of P. aeruginosa due to interactions with secreted trails". In: Physical Review Letters 117.17 (2016), p. 178102. 
[92] N. H. Mendelson, A. Bourque, K. Wilkening, K. R. Anderson, and J. C. Watkins. "Organized Cell Swimming Motions in Bacillus subtilis Colonies: Patterns of Short-Lived Whirls and Jets". In: Journal of Bacteriology 181.2 (1999), pp. 600609. DOI: 10.1128/JB.181.2.600-609.1999. eprint: https://jb.asm.org/content/ 181/2/600.full.pdf.

[93] X.-L. Wu and A. Libchaber. "Particle Diffusion in a Quasi-Two-Dimensional Bacterial Bath". In: Phys. Rev. Lett. 84 (13 Mar. 2000), pp. 3017-3020. DoI: 10.1103/PhysRevLett.84.3017.

[94] J. Kessler. "Collective behavior and dynamics of swimming bacteria". In: Bacteria as multicellular organisms (1997).

[95] C. Dombrowski, L. Cisneros, S. Chatkaew, R. E. Goldstein, and J. O. Kessler. "Self-Concentration and Large-Scale Coherence in Bacterial Dynamics". In: Phys. Rev. Lett. 93 (9 Aug. 2004), p. 098103. DOI: 10.1103/PhysRevLett.93.098103.

[96] I. Tuval, L. Cisneros, C. Dombrowski, C. W. Wolgemuth, J. O. Kessler, and R. E. Goldstein. "Bacterial swimming and oxygen transport near contact lines". In: Proceedings of the National Academy of Sciences 102.7 (2005), pp. 2277-2282. DOI: 10.1073/pnas.0406724102. eprint: https://www.pnas.org/content/102/7/ 2277.full.pdf.

[97] A. Sokolov, I. S. Aranson, J. O. Kessler, and R. E. Goldstein. "Concentration Dependence of the Collective Dynamics of Swimming Bacteria". In: Phys. Rev. Lett. 98 (15 Apr. 2007), p. 158102. DOI: 10.1103/PhysRevLett.98.158102.

[98] C. W. Wolgemuth. "Collective Swimming and the Dynamics of Bacterial Turbulence". In: Biophysical Journal 95.4 (2008), pp. 1564-1574. DOI: https://doi. org/10.1529/biophysj.107.118257.

[99] H. Moore, K. Dvorakova, N. Jenkins, and W. Breed. "Exceptional sperm cooperation in the wood mouse". In: Nature 418.6894 (2002), pp. 174-177.

[100] D. Zwicker, R. Seyboldt, C. A. Weber, A. A. Hyman, and F. Jülicher. "Growth and division of active droplets provides a model for protocells". In: Nature Physics 13.4 (2017), pp. 408-413.

[101] B. V. Hokmabad, K. A. Baldwin, C. Krüger, C. Bahr, and C. C. Maass. "Topological Stabilization and Dynamics of Self-Propelling Nematic Shells". In: Physical Review Letters 123.17 (2019), p. 178003.

[102] B. V. Hokmabad, R. Dey, M. Jalaal, D. Mohanty, M. Almukambetova, K. A. Baldwin, D. Lohse, and C. C. Maass. "Emergence of Bimodal Motility in Active Droplets". In: arXiv:2005.12721 [cond-mat, physics:physics] (2020). arXiv: 2005.12721 [cond-mat, physics:physics].

[103] C. Jin, B. Vajdi Hokmabad, K. A. Baldwin, and C. C. Maass. "Chemotactic Droplet Swimmers in Complex Geometries". In: J. Phys.: Condens. Mat. 30.5 (2018), p. 054003.

[104] B. V. Hokmabad, S. Saha, J. A. Canalejo, R. Golestanian, and C. C. Maass. "Quantitative characterization of chemorepulsive alignment-induced interactions in active emulsions". In: (2020). arXiv: 2012.05170 [cond-mat.soft]. 
[105] E. Lorenceau, A. S. Utada, D. R. Link, G. Cristobal, M. Joanicot, and D. A. Weitz. "Generation of Polymerosomes from Double-Emulsions". In: Langmuir 21.20 (2005), pp. 9183-9186.

[106] J. Petit, I. Polenz, J.-C. Baret, S. Herminghaus, and O. Bäumchen. "Vesicleson-a-Chip: A Universal Microfluidic Platform for the Assembly of Liposomes and Polymersomes". In: Eur. Phys. J. E. 39.6 (2016), p. 59.

[107] M. A. Augustin and Y. Hemar. "Nano- and Micro-Structured Assemblies for Encapsulation of Food Ingredients". In: Chem. Soc. Rev. 38.4 (2009), pp. 902912.

[108] S.-H. Kim, J. W. Kim, J.-C. Cho, and D. A. Weitz. "Double-Emulsion Drops with Ultra-Thin Shells for Capsule Templates". In: Lab Chip 11.18 (2011), pp. 3162-3166.

[109] L. Chen, Y. Li, J. Fan, H. K. Bisoyi, D. A. Weitz, and Q. Li. "Photoresponsive Monodisperse Cholesteric Liquid Crystalline Microshells for Tunable Omnidirectional Lasing Enabled by a Visible Light-Driven Chiral Molecular Switch". In: Adv. Opt. Mater. 2.9 (2014), pp. 845-848.

[110] S. Nagelberg et al. "Reconfigurable and Responsive Droplet-Based Compound Micro-Lenses". In: Nat. Commun. 8 (2017), p. 14673.

[111] L. D. Zarzar, V. Sresht, E. M. Sletten, J. A. Kalow, D. Blankschtein, and T. M. Swager. "Dynamically Reconfigurable Complex Emulsions via Tunable Interfacial Tensions". In: Nature 518.7540 (2015), pp. 520-524.

[112] Q. Zhang, S. Savagatrup, P. Kaplonek, P. H. Seeberger, and T. M. Swager. "Janus emulsions for the detection of bacteria". In: ACS central science 3.4 (2017), pp. 309-313.

[113] B. V. V. S. P. Kumar, A. J. Patil, and S. Mann. "Enzyme-Powered Motility in Buoyant Organoclay/DNA Protocells". In: Nat. Chem. 10.11 (2018), p. 1154.

[114] A. V. Singh, Z. Hosseinidoust, B.-W. Park, O. Yasa, and M. Sitti. "Microemulsion-Based Soft Bacteria-Driven Microswimmers for Active Cargo Delivery". In: ACS Nano 11.10 (2017), pp. 9759-9769.

[115] A. Joseph, C. Contini, D. Cecchin, S. Nyberg, L. Ruiz-Perez, J. Gaitzsch, G. Fullstone, X. Tian, J. Azizi, J. Preston, et al. "Chemotactic synthetic vesicles: Design and applications in blood-brain barrier crossing". In: Science Advances 3.8 (2017), e1700362.

[116] C. Krüger, G. Klös, C. Bahr, and C. C. Maass. "Curling Liquid Crystal Microswimmers: A Cascade of Spontaneous Symmetry Breaking". In: Physical Review Letters 117.4 (2016). DOI: 10.1103/PhysRevLett.117.048003.

[117] J. M. Brake, A. D. Mezera, and N. L. Abbott. "Effect of Surfactant Structure on the Orientation of Liquid Crystals at Aqueous-Liquid Crystal Interfaces". In: Langmuir 19.16 (2003), pp. 6436-6442.

[118] T. Lopez-Leon and A. Fernandez-Nieves. "Drops and Shells of Liquid Crystal". In: Colloid Polym. Sci. 289 (2011), pp. 345-359. 
[119] H. Mori, J. Eugene C. Gartland, J. R. Kelly, and P. J. Bos. "Multidimensional Director Modeling Using the Q Tensor Representation in a Liquid Crystal Cell and Its Application to the $\pi$ Cell with Patterned Electrodes". In: Jpn. J. Appl. Phys. 38.1R (1999), p. 135.

[120] M. Ravnik and S. Žumer. "Landau-de Gennes Modelling of Nematic Liquid Crystal Colloids". In: Liq. Cryst. 36.10-11 (2009), pp. 1201-1214.

[121] P.-G. De Gennes. "Short Range Order Effects in the Isotropic Phase of Nematics and Cholesterics". In: Mol. Cryst. Liq. Cryst. 12.3 (1971), pp. 193-214.

[122] P. P. Karat and N. V. Madhusudana. "Elasticity and Orientational Order in Some 4'-n-Alkyl-4-Cyanobiphenyls: Part II". In: Mol. Cryst. Liq. Cryst. 40.1 (1977), pp. 239-245.

[123] A. G. Chmielewski. "Viscosity Coefficients of Some Nematic Liquid Crystals". In: Mol. Cryst. Liq. Cryst. 132.3-4 (1986), pp. 339-352.

[124] N. D. Mermin. "The Topological Theory of Defects in Ordered Media". In: Rev. Mod. Phys. 51.3 (1979), pp. 591-648.

[125] T. C. Lubensky, D. Pettey, N. Currier, and H. Stark. "Topological Defects and Interactions in Nematic Emulsions". In: Phys. Rev. E. 57.1 (1998), pp. 610-625.

[126] P. Poulin and D. A. Weitz. "Inverted and Multiple Nematic Emulsions". In: Phys. Rev. E 57.1 (1998), pp. 626-637.

[127] P. Poulin, H. Stark, T. C. Lubensky, and D. A. Weitz. "Novel Colloidal Interactions in Anisotropic Fluids". In: Science 275.5307 (1997), p. 1770.

[128] S. Das, A. Garg, A. I. Campbell, J. Howse, A. Sen, D. Velegol, R. Golestanian, and S. J. Ebbens. "Boundaries Can Steer Active Janus Spheres". In: Nat. Commun. 6 (2015), p. 8999.

[129] J. Simmchen, J. Katuri, W. E. Uspal, M. N. Popescu, M. Tasinkevych, and S. Sánchez. "Topographical Pathways Guide Chemical Microswimmers". In: Nat. Commun. 7 (2016), p. 10598.

[130] D. Qin, Y. Xia, and G. M. Whitesides. "Soft Lithography for Micro- and Nanoscale Patterning". In: Nat. Protoc. 5.3 (2010), pp. 491-502.

[131] A. Schmit, L. Salkin, L. Courbin, and P. Panizza. "Commensurability-Driven Structural Defects in Double Emulsions Produced with Two-Step Microfluidic Techniques." In: Soft Matter 10.26 (2014), pp. 4743-8.

[132] J. C. Crocker and D. G. Grier. "Methods of Digital Video Microscopy for Colloidal Studies". In: J Colloid Interface Sci 179 (1996), pp. 298-310.

[133] W. Thielicke and E. Stamhuis. "PIVlab - Towards User-Friendly, Affordable and Accurate Digital Particle Image Velocimetry in MATLAB". In: Journal of Open Research Software 2.1 (2014), e30.

[134] H. Wioland, F. G. Woodhouse, J. Dunkel, and R. E. Goldstein. "Ferromagnetic and Antiferromagnetic Order in Bacterial Vortex Lattices". In: Nat Phys 12 (2016), pp. 341-345.

[135] R. N. Bearon. "Helical Swimming Can Provide Robust Upwards Transport for Gravitactic Single-Cell Algae; a Mechanistic Model". In: J. Math. Biol. 66.7 (2013), pp. 1341-1359. 
[136] M. Rossi, G. Cicconofri, A. Beran, G. Noselli, and A. DeSimone. "Kinematics of Flagellar Swimming in Euglena Gracilis: Helical Trajectories and Flagellar Shapes". In: Proceedings of the National Academy of Sciences 114.50 (2017), pp. 13085-13090.

[137] R. Stocker. "Reverse and Flick: Hybrid Locomotion in Bacteria." In: Proc. Natl. Acad. Sci. U. S. A. 108.7 (2011), pp. 2635-6.

[138] L. Cisneros, C. Dombrowski, R. E. Goldstein, and J. O. Kessler. "Reversal of Bacterial Locomotion at an Obstacle". In: Physical Review E 73.3 (2006), p. 030901.

[139] K. Y. Wan and R. E. Goldstein. "Time Irreversibility and Criticality in the Motility of a Flagellate Microorganism". In: Physical Review Letters 121.5 (2018), p. 058103.

[140] W. Gilpin, V. N. Prakash, and M. Prakash. "Vortex Arrays and Ciliary Tangles Underlie the Feeding-Swimming Trade-off in Starfish Larvae". In: Nature Physics 13.4 (2017), pp. 380-386.

[141] A. C. H. Tsang, A. T. Lam, and I. H. Riedel-Kruse. "Polygonal Motion and Adaptable Phototaxis via Flagellar Beat Switching in the Microswimmer Euglena Gracilis". In: Nature Physics 14.12 (2018), pp. 1216-1222.

[142] K. Son, J. S. Guasto, and R. Stocker. "Bacteria Can Exploit a Flagellar Buckling Instability to Change Direction". In: Nature Physics 9.8 (2013), pp. 494-498.

[143] N. Figueroa-Morales, A. Rivera, R. Soto, A. Lindner, E. Altshuler, and É. Clément. "E. Coli "Super-Contaminates" Narrow Ducts Fostered by Broad Run-Time Distribution". In: Science Advances 6.11 (2020), eaay0155.

[144] G. H. Wadhams and J. P. Armitage. "Making Sense of It All: Bacterial Chemotaxis". In: Nature Reviews Molecular Cell Biology 5.12 (2004), pp. 1024-1037.

[145] E. Perez Ipiña, S. Otte, R. Pontier-Bres, D. Czerucka, and F. Peruani. "Bacteria Display Optimal Transport near Surfaces". In: Nature Physics 15.6 (2019), pp. 610-615.

[146] Ò. Guadayol, K. L. Thornton, and S. Humphries. "Cell Morphology Governs Directional Control in Swimming Bacteria". In: Scientific Reports 7.1 (2017), p. 2061.

[147] J. Palacci, S. Sacanna, A. Abramian, J. Barral, K. Hanson, A. Y. Grosberg, D. J. Pine, and P. M. Chaikin. "Artificial Rheotaxis". In: Science Advances 1.4 (2015), e1400214.

[148] J. Katuri, W. E. Uspal, J. Simmchen, A. Miguel-López, and S. Sánchez. "CrossStream Migration of Active Particles". In: Science Advances 4.1 (2018), eaao1755.

[149] B. Liebchen and H. Löwen. "Synthetic Chemotaxis and Collective Behavior in Active Matter". In: Accounts of Chemical Research 51.12 (2018), pp. 2982-2990.

[150] B. ten Hagen, F. Kümmel, R. Wittkowski, D. Takagi, H. Löwen, and C. Bechinger. "Gravitaxis of Asymmetric Self-Propelled Colloidal Particles". In: Nature Communications 5.1 (2014), p. 4829. 
[151] M. Suga, S. Suda, M. Ichikawa, and Y. Kimura. "Self-Propelled Motion Switching in Nematic Liquid Crystal Droplets in Aqueous Surfactant Solutions". In: Physical Review E 97.6 (2018), p. 062703.

[152] A. Izzet, P. G. Moerman, P. Gross, J. Groenewold, A. D. Hollingsworth, J. Bibette, and J. Brujic. "Tunable Persistent Random Walk in Swimming Droplets". In: Physical Review X 10.2 (2020), p. 021035.

[153] T. Bhattacharjee and S. S. Datta. "Bacterial Hopping and Trapping in Porous Media". In: Nature Communications 10.1 (2019), p. 2075.

[154] W.-F. Hu, T.-S. Lin, S. Rafai, and C. Misbah. "Chaotic Swimming of Phoretic Particles". In: Physical Review Letters 123.23 (2019), p. 238004.

[155] L. G. Leal. Advanced Transport Phenomena: Fluid Mechanics and Convective Transport Processes. Cambridge Series in Chemical Engineering. Cambridge: Cambridge University Press, 2007.

[156] G. Slade. "Self-Avoiding Walks". In: The Mathematical Intelligencer 16.1 (1994), pp. 29-35.

[157] J. Najafi, M. R. Shaebani, T. John, F. Altegoer, G. Bange, and C. Wagner. "Flagellar Number Governs Bacterial Spreading and Transport Efficiency". In: Science Advances 4.9 (2018), eaar6425.

[158] R. Golestanian. "Anomalous Diffusion of Symmetric and Asymmetric Active Colloids". In: Physical Review Letters 102.18 (2009), p. 188305.

[159] C. R. Reid, T. Latty, A. Dussutour, and M. Beekman. "Slime Mold Uses an Externalized Spatial "Memory" to Navigate in Complex Environments". In: Proceedings of the National Academy of Sciences 109.43 (2012), pp. 17490-17494.

[160] A. G. Cherstvy, O. Nagel, C. Beta, and R. Metzler. "Non-Gaussianity, Population Heterogeneity, and Transient Superdiffusion in the Spreading Dynamics of Amoeboid Cells". In: Physical Chemistry Chemical Physics 20.35 (2018), pp. 23034-23054.

[161] M. Schmitt and H. Stark. "Swimming active droplet: A theoretical analysis". In: Europhysics Letters (EPL) 101.4 (Feb. 2013), p. 44008. DOI: 10.1209/0295$5075 / 101 / 44008$.

[162] C. H. Meredith, P. G. Moerman, J. Groenewold, Y.-J. Chiu, W. K. Kegel, A. van Blaaderen, and L. D. Zarzar. "Predator-Prey Interactions between Droplets Driven by Nonreciprocal Oil Exchange". In: arXiv:1912.10102 [cond-mat] (2019). arXiv: 1912.10102 [cond-mat].

[163] M. Jabbarzadeh and H. C. Fu. "Viscous Constraints on Microorganism Approach and Interaction". In: Journal of Fluid Mechanics 851 (2018), pp. 715-738.

[164] B. Nasouri and R. Golestanian. "Exact Phoretic Interaction of Two Chemically Active Particles". In: Physical Review Letters 124.16 (2020), p. 168003. arXiv: 2001.07576.

[165] K. Lippera, M. Benzaquen, and S. Michelin. "Bouncing, Chasing, or Pausing: Asymmetric Collisions of Active Droplets". In: Physical Review Fluids 5.3 (2020), p. 032201. 
[166] F. Yang, B. Rallabandi, and H. A. Stone. "Autophoresis of Two Adsorbing/Desorbing Particles in an Electrolyte Solution". In: Journal of Fluid Mechanics 865 (2019), pp. 440-459.

[167] Y. Couder, S. Protière, E. Fort, and A. Boudaoud. "Walking and Orbiting Droplets". In: Nature 437.7056 (2005), pp. 208-208.

[168] M. Hubert, S. Perrard, M. Labousse, N. Vandewalle, and Y. Couder. "Tunable Bimodal Explorations of Space from Memory-Driven Deterministic Dynamics". In: Physical Review E 100.3 (2019), p. 032201.

[169] R. N. Valani, A. C. Slim, and T. Simula. "Superwalking Droplets". In: Physical Review Letters 123.2 (2019), p. 024503.

[170] W. Gilpin, V. N. Prakash, and M. Prakash. "Flowtrace: Simple Visualization of Coherent Structures in Biological Fluid Flows". In: Journal of Experimental Biology 220.19 (2017), pp. 3411-3418.

[171] E. M. Purcell. "Life at low Reynolds number". In: American Journal of Physics 45.1 (Jan. 1977), p. 3. DOI: 10.1119/1.10903.

[172] J. Palacci, C. Cottin-Bizonne, C. Ybert, and L. Bocquet. "Sedimentation and Effective Temperature of Active Colloidal Suspensions". In: Physical Review Letters 105.8 (Aug. 2010), p. 088304. DOI: 10.1103/PhysRevLett.105.088304.

[173] I. Buttinoni, G. G. Volpe, F. Kümmel, G. G. Volpe, and C. Bechinger. "Active Brownian motion tunable by light". In: Journal of Physics: Condensed Matter 24.28 (2012), p. 284129. DOI: 10.1088/0953-8984/24/28/284129.

[174] R. F. Ismagilov, A. Schwartz, N. Bowden, and G. M. Whitesides. "Autonomous Movement and Self-Assembly". In: Angewandte Chemie International Edition 41.4 (Feb. 2002), pp. 652-654. DOI: 10.1002/1521-3773(20020215)41:4<652:: AID-ANIE652>3.0.CO;2-U.

[175] A. A. Solovev, Y. Mei, E. Bermúdez Ureña, G. Huang, and O. G. Schmidt. "Catalytic Microtubular Jet Engines Self-Propelled by Accumulated Gas Bubbles". In: Small 5.14 (July 2009), pp. 1688-92. DOI: 10.1002/smll.200900021.

[176] A. Snezhko, M. Belkin, I. S. Aranson, and W.-K. Kwok. "Self-Assembled Magnetic Surface Swimmers". In: Physical Review Letters 102.11 (Mar. 2009), p. 118103. DOI: 10.1103/PhysRevLett.102.118103.

[177] W. F. Paxton, K. C. Kistler, C. C. Olmeda, A. Sen, S. K. St Angelo, Y. Cao, T. E. Mallouk, P. E. Lammert, and V. H. Crespi. "Catalytic nanomotors: autonomous movement of striped nanorods." In: Journal of the American Chemical Society 126.41 (Oct. 2004), pp. 13424-31. DOI: 10.1021/ja047697z.

[178] C. Bechinger, R. Di Leonardo, H. Löwen, C. Reichhardt, G. Volpe, and G. Volpe. "Active Particles in Complex and Crowded Environments". In: Reviews of Modern Physics 88.4 (Nov. 2016), p. 045006. DOI: 10.1103/RevModPhys.88.045006.

[179] J. Elgeti, R. G. Winkler, and G. Gompper. "Physics of microswimmers-single particle motion and collective behavior: a review." In: Reports on Progress in Physics 78.5 (May 2015), p. 056601. DOI: 10.1088/0034-4885/78/5/056601. 
[180] B. ten Hagen, R. Wittkowski, D. Takagi, F. Kümmel, C. Bechinger, and H. Löwen. "Can the self-propulsion of anisotropic microswimmers be described by using forces and torques?" In: Journal of Physics: Condensed Matter 27.19 (May 2015), p. 194110. DOI: 10.1088/0953-8984/27/19/194110.

[181] P. Romanczuk, M. Bär, W. Ebeling, B. Lindner, and L. Schimansky-Geier. "Active Brownian particles: From individual to collective stochastic dynamics". In: The European Physical Journal Special Topics 202.1 (2012), pp. 1-162. DOI: 10.1140/epjst/e2012-01529-y.

[182] A. Wysocki, J. Elgeti, and G. Gompper. "Giant adsorption of microswimmers: Duality of shape asymmetry and wall curvature". In: Physical Review E 91.5 (May 2015), p. 050302. DOI: 10.1103/PhysRevE.91.050302.

[183] R. Wittkowski and H. Löwen. "Self-propelled Brownian spinning top: dynamics of a biaxial swimmer at low Reynolds numbers." In: Physical Review E $85.2 \mathrm{Pt}$ 1 (Feb. 2012), p. 021406. DOI: 10.1103/PhysRevE.85.021406.

[184] H. H. Wensink, V. Kantsler, R. E. Goldstein, and J. Dunkel. "Controlling active self-assembly through broken particle-shape symmetry". In: Physical Review E - Statistical, Nonlinear, and Soft Matter Physics 89.1 (2014). DOI: 10.1103/PhysRevE.89.010302.

[185] M. Mijalkov and G. Volpe. "Sorting of chiral microswimmers". en. In: Soft Matter 9.28 (June 2013), p. 6376. DOI: 10.1039/c3sm27923e.

[186] V. Kantsler, J. Dunkel, M. Polin, and R. E. Goldstein. "Ciliary contact interactions dominate surface scattering of swimming eukaryotes". In: Proceedings of the National Academy of Sciences 110.4 (2013), pp. 1187-1192. DOI: 10.1073/pnas.1210548110.

[187] P. Denissenko, V. Kantsler, D. J. Smith, and J. Kirkman-Brown. "Human spermatozoa migration in microchannels reveals boundary-following navigation". In: Proceedings of the National Academy of Sciences 109.21 (May 2012), pp. 800710. DOI: $10.1073 /$ pnas. 1202934109 .

[188] K. Schaar, A. Zöttl, and H. Stark. "Detention Times of Microswimmers Close to Surfaces: Influence of Hydrodynamic Interactions and Noise". In: Physical Review Letters 115.3 (2015). DOI: 10.1103/PhysRevLett.115.038101.

[189] M. Contino, E. Lushi, I. Tuval, V. Kantsler, and M. Polin. "Microalgae Scatter off Solid Surfaces by Hydrodynamic and Contact Forces". In: Physical Review Letters 115.25 (2015). DOI: 10.1103/PhysRevLett.115.258102.

[190] D. Takagi, J. Palacci, A. B. Braunschweig, M. J. Shelley, and J. Zhang. "Hydrodynamic capture of microswimmers into sphere-bound orbits". In: Soft Matter 10.1748 (2014), pp. 3-8. DOI: 10.1039/c3sm52815d.

[191] S. E. Spagnolie and E. Lauga. "Hydrodynamics of self-propulsion near a boundary: predictions and accuracy of far-field approximations". English. In: Journal of Fluid Mechanics 700 (Apr. 2012), pp. 105-147. DOI: 10.1017/jfm.2012.101.

[192] O. Sipos, K. Nagy, R. Di Leonardo, and P. Galajda. "Hydrodynamic Trapping of Swimming Bacteria by Convex Walls". In: Physical Review Letters 114.25 (June 2015), p. 258104. DOI: 10.1103/PhysRevLett.114.258104. 
[193] J. S. Lintuvuori, A. T. Brown, K. Stratford, and D. Marenduzzo. "Hydrodynamic oscillations and tunable swimming speed in squirmers close to repulsive walls". In: Soft Matter 1 (2015), pp. 1-6. DOI: 10.1039/C6SM01353H.

[194] V. Sourjik and N. S. Wingreen. Responding to chemical gradients: Bacterial chemotaxis. 2012. DOI: 10.1016/j.ceb.2011.11.008.

[195] H. C. Berg and D. a. Brown. "Chemotaxis in Escherichia coli analysed by three-dimensional tracking." In: Nature 239.5374 (1972), pp. 500-504. DOI: 10.1038/239500a0.

[196] J. Adler. "Chemotaxis in Bacteria". In: Science 153.3737 (1966).

[197] L. Xie, T. Altindal, S. Chattopadhyay, and X.-L. Wu. "Bacterial flagellum as a propeller and as a rudder for efficient chemotaxis". In: Proceedings of the National Academy of Sciences 108.6 (Mar. 2011), pp. 2246-51. DOI: 10.1073/ pnas.1011953108.

[198] L. Alvarez, B. M. Friedrich, G. Gompper, and U. B. Kaupp. "The computational sperm cell". In: Trends in Cell Biology 24.3 (Mar. 2014), pp. 198-207. DOI: 10.1016/j.tcb.2013.10.004.

[199] H. Levine, D. A. Kessler, and W.-J. Rappel. "Directional sensing in eukaryotic chemotaxis: a balanced inactivation model." In: Proceedings of the National Academy of Sciences 103.26 (June 2006), pp. 9761-9766. DOI: 10.1073/pnas. 0601302103.

[200] D. B. Dusenbery. "Spatial sensing of stimulus gradients can be superior to temporal sensing for free-swimming bacteria." In: Biophysical journal 74.5 (1998), pp. 2272-7. DOI: 10.1016/S0006-3495(98)77936-6.

[201] M. Mijalkov, A. McDaniel, J. Wehr, and G. Volpe. "Engineering Sensorial Delay to Control Phototaxis and Emergent Collective Behaviors". In: Physical Review X 6.1 (Jan. 2016), p. 011008. DOI: 10.1103/PhysRevX.6.011008.

[202] J. Taktikos, V. Zaburdaev, and H. Stark. "Modeling a self-propelled autochemotactic walker". In: Physical Review E 84.4 (Oct. 2011), p. 041924. DOI: 10.1103/ PhysRevE.84.041924.

[203] E. O. Budrene and H. C. Berg. "Dynamics of formation of symmetrical patterns by chemotactic bacteria". In: Nature 376.6535 (July 1995), pp. 49-53. DOI: 10.1038/376049a0.

[204] P. Galajda, J. Keymer, P. Chaikin, and R. Austin. "A wall of funnels concentrates swimming bacteria". In: Journal of Bacteriology 189.23 (2007), pp. 8704-8707. DOI: 10.1128/JB.01033-07.

[205] G. Lambert, D. Liao, and R. H. Austin. "Collective Escape of Chemotactic Swimmers through Microscopic Ratchets". In: Physical Review Letters 104.16 (Apr. 2010), p. 168102. DOI: 10.1103/PhysRevLett.104.168102.

[206] S. Park, P. M. Wolanin, E. a. Yuzbashyan, H. Lin, N. C. Darnton, J. B. Stock, P. Silberzan, and R. Austin. "Influence of topology on bacterial social interaction". In: Proceedings of the National Academy of Sciences 100.24 (Nov. 2003), pp. 13910-13915. DOI: 10.1073/pnas.1935975100. 
[207] M. Gharasoo, F. Centler, I. Fetzer, and M. Thullner. "How the chemotactic characteristics of bacteria can determine their population patterns". In: Soil Biology and Biochemistry 69 (2014), pp. 346-358. DOI: 10.1016/j.soilbio.2013. 11.019 .

[208] D. Qin, Y. Xia, and G. M. Whitesides. "Soft lithography for micro- and nanoscale patterning". In: Nature Protocols 5.3 (Mar. 2010), pp. 491-502. DOI: 10.1038/ nprot.2009.234.

[209] T. Thorsen, R. W. Roberts, F. H. Arnold, and S. R. Quake. "Dynamic Pattern Formation in a Vesicle-Generating Microfluidic Device". In: Physical Review Letters 86.18 (Apr. 2001), pp. 4163-4166. DOI: 10.1103/PhysRevLett.86.4163.

[210] S. E. Spagnolie, G. R. Moreno-Flores, D. Bartolo, and E. Lauga. "Geometric capture and escape of a microswimmer colliding with an obstacle". In: Soft Matter 11.17 (2015), pp. 3396-3411. DOI: 10.1039/C4SM02785J.

[211] T. Bhattacharjee, D. B. Amchin, J. A. Ott, F. Kratz, and S. S. Datta. "Chemotactic Migration of Bacteria in Porous Media". In: bioRxiv (2020), p. 2020.08.10.244731.

[212] E. Budrene and H. Berg. "Dynamics of Formation of Symmetrical Patterns by Chemotactic Bacteria". In: Nature 376.6535 (1995), p. 49.

[213] M. P. Brenner, L. S. Levitov, and E. O. Budrene. "Physical Mechanisms for Chemotactic Pattern Formation by Bacteria". In: Biophysical Journal 74.4 (1998), pp. 1677-1693.

[214] J. Agudo-Canalejo and R. Golestanian. "Active Phase Separation in Mixtures of Chemically Interacting Particles". In: Phys. Rev. Lett. 123.1 (2019), p. 018101.

[215] R. Golestanian. "Collective Behavior of Thermally Active Colloids". In: Phys. Rev. Lett. 108 (3 Jan. 2012), p. 038303. DoI: 10.1103/PhysRevLett.108.038303.

[216] C. Jin, J. Vachier, S. Bandyopadhyay, T. Macharashvili, and C. C. Maass. "Fine Balance of Chemotactic and Hydrodynamic Torques: When Microswimmers Orbit a Pillar Just Once". In: Physical Review E 100.4 (2019), p. 040601.

[217] A. Gelimson, K. Zhao, C. K. Lee, W. T. Kranz, G. C. L. Wong, and R. Golestanian. "Multicellular Self-Organization of P. aeruginosa due to Interactions with Secreted Trails". In: Phys. Rev. Lett. 117 (17 Oct. 2016), p. 178102. DOI: 10.1103/PhysRevLett.117.178102.

[218] S. Saha, R. Golestanian, and S. Ramaswamy. "Clusters, Asters, and Collective Oscillations in Chemotactic Colloids". In: Physical Review E 89.6 (2014), p. 062316.

[219] S. Saha, S. Ramaswamy, and R. Golestanian. "Pairing, Waltzing and Scattering of Chemotactic Active Colloids". In: New Journal of Physics 21.6 (2019), p. 063006.

[220] E. R. Weeks, J. C. Crocker, A. C. Levitt, A. Schofield, and D. A. Weitz. "ThreeDimensional Direct Imaging of Structural Relaxation Near the Colloidal Glass Transition". In: Science 287.5453 (2000), pp. 627-631.

[221] P. N. Pusey and W. van Megen. "Phase Behaviour of Concentrated Suspensions of Nearly Hard Colloidal Spheres". In: Nature 320.6060 (1986), pp. 340-342. 
[222] K. Lippera, M. Benzaquen, and S. Michelin. "Alignment and scattering of colliding active droplets". In: Soft Matter (2020).

[223] G. Bradski. "The OpenCV Library". In: Dr Dobbs J. Softw. Tools 25 (2000), pp. 120-125.

[224] F. Pedregosa et al. "Scikit-Learn: Machine Learning in Python". In: Journal of Machine Learning Research 12.85 (2011), pp. 2825-2830.

[225] D. Comaniciu and P. Meer. "Mean Shift: A Robust Approach toward Feature Space Analysis". In: IEEE Transactions on Pattern Analysis and Machine Intelligence 24.5 (2002), pp. 603-619.

[226] M. Sapozhnikov, Y. V. Tolmachev, I. Aranson, and W.-K. Kwok. "Dynamic selfassembly and patterns in electrostatically driven granular media". In: Physical review letters 90.11 (2003), p. 114301.

[227] A. Snezhko and I. S. Aranson. "Magnetic manipulation of self-assembled colloidal asters". In: Nature materials 10.9 (2011), pp. 698-703.

[228] M. C. Marchetti, J.-F. Joanny, S. Ramaswamy, T. B. Liverpool, J. Prost, M. Rao, and R. A. Simha. "Hydrodynamics of soft active matter". In: Reviews of Modern Physics 85.3 (2013), p. 1143.

[229] S. Ramaswamy. "Active fluids". In: Nature Reviews Physics (2019), pp. 1-3.

[230] F. J. Schwarzendahl and M. G. Mazza. "Maximum in Density Heterogeneities of Active Swimmers". In: Soft Matter 14.23 (2018), pp. 4666-4678.

[231] R. Singh and R. Adhikari. "Universal hydrodynamic mechanisms for crystallization in active colloidal suspensions". In: Physical review letters 117.22 (2016), p. 228002.

[232] A. P. Petroff, X.-L. Wu, and A. Libchaber. "Fast-Moving Bacteria Self-Organize into Active Two-Dimensional Crystals of Rotating Cells". In: Physical Review Letters 114.15 (Apr. 2015), p. 158102. DOI: 10.1103/PhysRevLett.114.158102.

[233] P. G. Moerman, H. W. Moyses, E. B. Van Der Wee, D. G. Grier, A. Van Blaaderen, W. K. Kegel, J. Groenewold, and J. Brujic. "Solute-mediated interactions between active droplets". In: Physical Review E 96.3 (2017), p. 032607.

[234] F. Rühle, J. Blaschke, J.-T. Kuhr, and H. Stark. "Gravity-Induced Dynamics of a Squirmer Microswimmer in Wall Proximity". In: New Journal of Physics 20.2 (2018), p. 025003.

[235] D. Das and E. Lauga. "Transition to Bound States for Bacteria Swimming near Surfaces". In: Physical Review E 100.4 (2019), p. 043117.

[236] M. Lighthill. "On the squirming motion of nearly spherical deformable bodies through liquids at very small Reynolds numbers". In: Communications on Pure and Applied Mathematics 5.2 (1952), pp. 109-118. 


\section{Acknowledgements}

I was very fortunate to have the opportunity to pursue my research career toward Ph.D. at the Max Planck Institute for Dynamics and Self-Organization. My deepest gratitude goes to my advisor Dr. Corinna Maass who introduced me to the exciting world of active matter. Thank you for your trust and support. I learned a lot from you both scientifically and non-scientifically.

I would like to express my sincere gratitude to Prof. Stephan Herminghaus for giving me the great opportunity to work in his department. His passion for research has created a stimulating atmosphere in our department of Dynamics of Complex Fluids (DCF) and will always be a source of inspiration for me.

Many thanks to Dr. Christian Bahr, for introducing me to the physics of liquid crystals. In particular, I appreciate his crucial contributions in the simulations of the liquid crystal shells in Chapter 2.

It was an honor to have Prof. Annette Zippelius on my thesis advisory committee. I appreciate her guidance and value feedback. I am grateful to the members of my defense committee and in particular Prof. Stefan Klumpp for reviewing my thesis.

I was very fortunate to have the opportunity of establishing collaborations with world-renowned expert groups. I thank Prof. Detlef Lohse and Dr. Maziyar Jalaal for hosting me at the University of Twente which led to a very fruitful collaboration presented in Chapter 3. Many thanks are due to Prof. Ramin Golestanian and his group members Jaime Agudo-Canalejo and Suropriya Saha for scientific discussions and their valuable theoretical contribution to the study in Chapter 5. I would like to thank Prof. David Officer and his group for hosting me at the University of Wollongong. I am also thankful to Prof. Marco Mazza and Dr. Fabian Schwarzendahl for their theoretical contributions and stimulating discussions.

I would like to express my sincere gratitude to all my colleagues at DCF. From my first day, they accepted me and let me feel welcome. I am truly grateful to Monika Teuteberg for her tireless support. I thank Dr. Kristian Hantke for the scientific discussions and the crucial technical support, and Julien Petit for his assistance with 
microfluidics, and I thank both for teaching me how to play Foosball. I also appreciate the support from Dr. Guido Schriever and Thomas Eggers.

I am thankful to my past and current groupmates. In particular, Dr. Carsten Krüger for training me and getting me started with active droplets, Prashanth Ramesh for his assistance with the measurements, and my dearest friend Dr. Ranabir Dey for the many things that I learned from him. I thank Dr. Chenyu Jin, Dr. Kyle Baldwin, and the master's and bachelor's students and interns that I had the chance to work with. I appreciate the scientific input from Babak Nasouri.

I was very fortunate to meet so many great people and make friends with them during my doctoral studies. Because of you guys I feel at home, here in Göttingen. Sayedeh, I am grateful to have you by my side.

I am forever thankful for the support that I have received from my family. Mom, Dad, and Siamak, I wish I could have you by my side at least in my defense. This thesis is dedicated to you. 